\title{
WestVirginiaUniversity
}

THE RESEARCH REPOSITORY @ WVU

Graduate Theses, Dissertations, and Problem Reports

2002

\section{Analysis of complex ventilation networks in multiple fan coal mines}

Khaled Ali El-Nagdy

West Virginia University

Follow this and additional works at: https://researchrepository.wvu.edu/etd

\section{Recommended Citation}

El-Nagdy, Khaled Ali, "Analysis of complex ventilation networks in multiple fan coal mines" (2002).

Graduate Theses, Dissertations, and Problem Reports. 2413.

https://researchrepository.wvu.edu/etd/2413

This Dissertation is protected by copyright and/or related rights. It has been brought to you by the The Research Repository @ WVU with permission from the rights-holder(s). You are free to use this Dissertation in any way that is permitted by the copyright and related rights legislation that applies to your use. For other uses you must obtain permission from the rights-holder(s) directly, unless additional rights are indicated by a Creative Commons license in the record and/ or on the work itself. This Dissertation has been accepted for inclusion in WVU Graduate Theses, Dissertations, and Problem Reports collection by an authorized administrator of The Research Repository @ WVU.

For more information, please contact researchrepository@mail.wvu.edu. 


\title{
Analysis of Complex Ventilation Networks \\ In Multiple Fan Coal Mines
}

\author{
Khaled Ali El-Nagdy
}

\author{
Dissertation Submitted to the \\ College of Engineering and Mineral Resources \\ at West Virginia University \\ In Partial Fulfillment of the Requirements \\ for the Degree of
}

Doctor of Philosophy
In

Engineering

Lloyd English, Ph.D., Chair

Syd Peng, Ph.D.

Yi Luo, Ph.D.

John Loth, Ph.D.

Ian Christie, Ph.D.

Department of Mining Engineering

Morgantown, West Virginia

2002

Keywords: Mine Ventilation, Complex Ventilation Networks, Multiple Fan analysis, Coal Mines, Hardy Cross, Newton-Raphson, Switching Parameters

Copyright 2002 Khaled El-Nagdy 


\section{ABSTRACT \\ Analysis of Complex Ventilation Networks In Multiple Fan Coal Mines Khaled El-Nagdy}

Coal mines require very effective and proper ventilation systems not only to keep the levels of explosive, harmful gases and dust to a safe level but also to deliver enough fresh air for miners to breathe. Depending upon the size of the mine and the quantity of gases and dust in this mine, it could be ventilated by using a single fan or several fans. There are two types of fans used in mines: vane axial and centrifugal.

In multiple-fan systems, each individual fan influences the performance of the whole system, including the other fans. Each fan in the system has its own mine characteristic curve or subsystem curve. Thus the present of an axial flow fan or fans inside each subsystem turns the resistance factor of the subsystem into a variable. Thus the relationship between the airflow and the pressure consumed through the subsystem does not strictly follow Atkinson equation. The shape of the subsystem curve and the number of the operating points in the system depend on the number and type of fans inside the subsystem. The shape of this subsystem characteristic curve could have sharp turning points and its shape may not be fixed as in the single fan system.

Both the implemented Hardy Cross method and damped Newton-Raphson method using switching parameters are found to be good tools to trace these subsystem characteristic curves. The modified Hardy Cross method is faster and more flexible than the modified damped Newton-Raphson method, especially when solving large networks. 
In Loving Memory of My Parents 


\section{ACKNOWLEDGEMENT}

I would like to express my sincere gratitude to the examining committee members: Dr. John. Loth, Department of Mechanical and Aerospace Engineering, Dr. Ian Christie, Mathematics Department, Dr. Yi Luo and Dr. Syd Peng, Mining Engineering Department, for their support and guidance any time I needed it. I would also like to thank Dr. Lloyd English, the chairman of the committee, for his continuous support. Special thank to Dr. Y.J Wang for his help, continuous support and encouragement throughout the first year of this study and since his retirement from Mining Engineering Department.

I am also grateful to Dr. Anthony Iannacchione and Mr. Robert Krog, National Institute for Occupational Safety and Health (NIOSH). Mr. Dan Alexander, Mining Engineering Department, Mr. Paul Gorder, Consolidation Coal Company, Mr. Mark Schroeder and Mr. John Urosek, MSHA, US Department of Labor, Mr. Larry Benet and Franck Catron, US Steel Mining Company, for their support and providing me with all information and data that I need in this study.

I would like to dedicate this dissertation to my family, especially my wife, for their understanding, encouragement, and full support of my graduate study. Finally I would like to remember my late parents for their inspiring words before their death; " we wish that we could have seen you become a Doctor". I hope I have fulfilled your dreams. 


\section{TABLE OF CONTENTS}

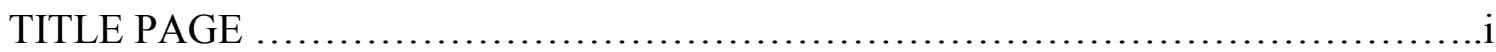

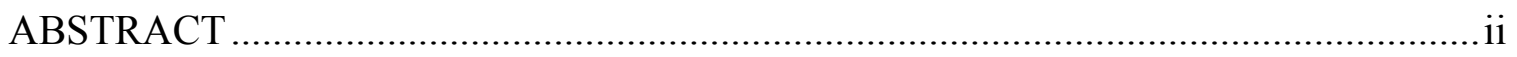

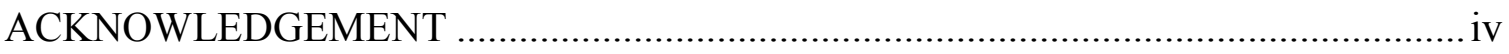

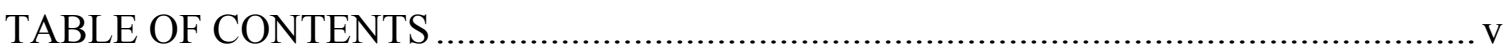

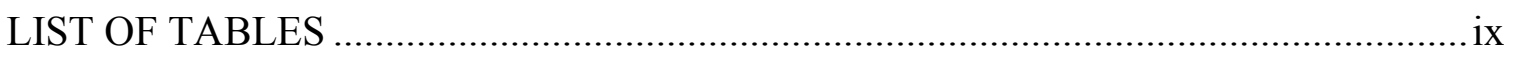

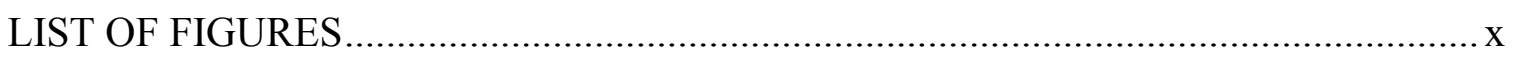

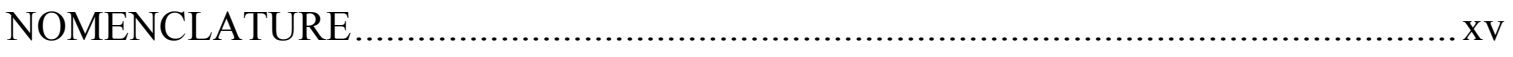

CHAPTER 1. INTRODUCTION AND PROBLEM STATEMENT ........................... 1

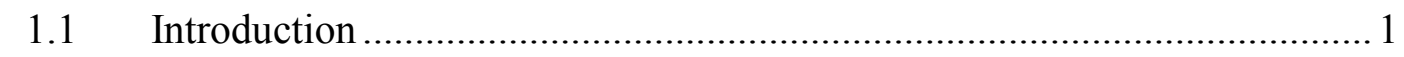

$1.2 \quad$ Problem Statement .....................................................................

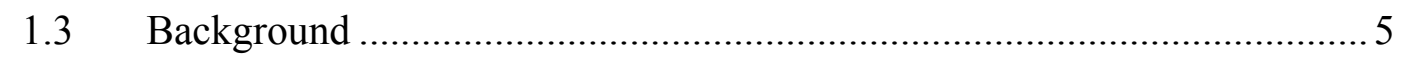

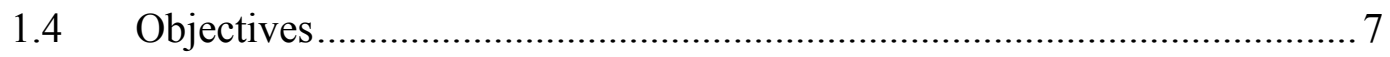

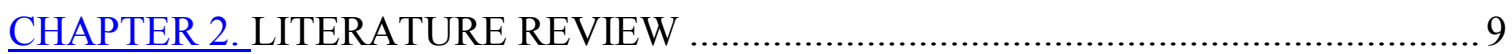

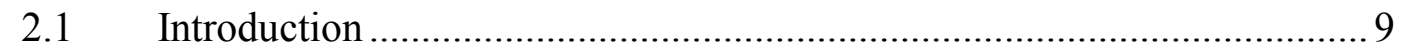

$2.2 \quad$ Basic Laws Applied in the Analysis of Mine Ventilation Systems ......... 10

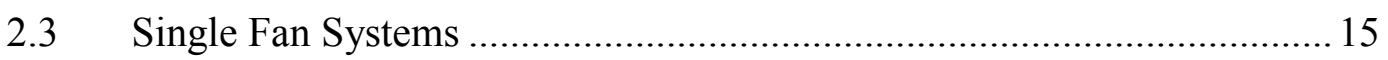

$2.4 \quad$ Multiple Fan Systems ............................................................ 16

$2.5 \quad$ Graphical Solution of Mine Ventilation Networks ............................ 19

$2.6 \quad$ Numerical Solution of Mine Ventilation Networks ...........................20

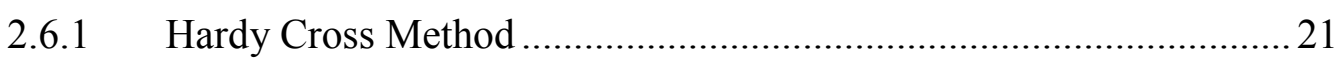

$2.6 .2 \quad$ Newton-Raphson Method .................................................. 27

$2.6 .3 \quad$ Other Mathematical Methods................................................ 30 
$2.7 \quad$ Review of Numerical Solutions for Multiple-Fan System..................... 33

$2.8 \quad$ Conclusions Based Upon Literature Review ................................... 37

CHAPTER 3. MODIFIED HARDY CROSS AND NEWTON METHODS .................39

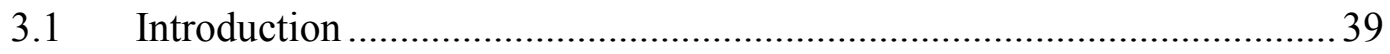

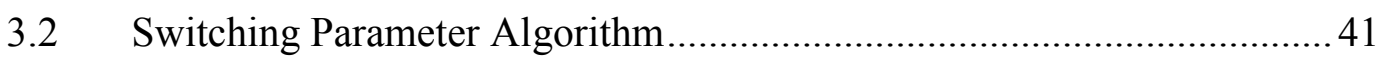

$3.3 \quad$ Cutset and Cutset Operations ................................................. 44

3.4 Modified Hardy Cross Method .................................................... 46

$3.5 \quad$ Modified Damped Newton Raphson Method ..................................... 52

3.6 Application of the Algorithms to an Administrative Example of Multiple

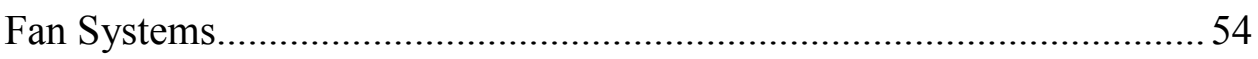

3.6.1 Approximation of Fan Characteristic Curves ............................5

3.7 Relation Between Airflow and Subsystem's Resistance in Multiple Fan

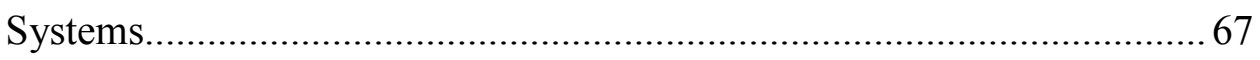

3.8 Comparison Between the Performance of Hardy Cross and Damped

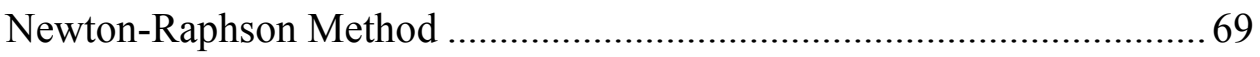

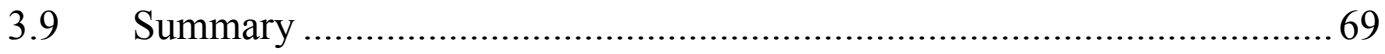

CHAPTER 4. OPERATING CHARACTERISTICS OF MULTIPLE FAN SYSTEMS. 71

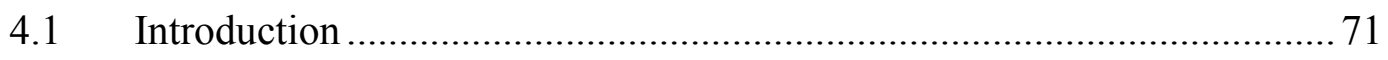

4.2 Effect of Resistance Factors on the Shape of the Subsystem Characteristic

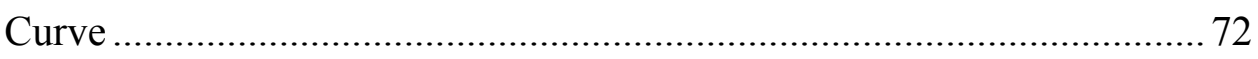

4.2.1 Impact of the Fan Branch's Resistance Changes on the Shape of the

Subsystem Characteristic Curve …......................................... 72 
4.2.2 Impact of the Regular Branch's Resistance Changes on the Shape of

the Subsystem Characteristic Curve ............................................ 78

$4.3 \quad$ Effect of the Topology of the Network on the Shape of the Subsystem

Characteristic Curve ...................................................................... 83

$4.4 \quad$ Effect of Number of Fans in the System on the Shape of the Subsystems91

4.5 Effect of Type of Fans in the System on the Shape of the Subsystems .. 102

4.6 Effect of Fan Speed in the System on the Shape of the Subsystem ........ 103

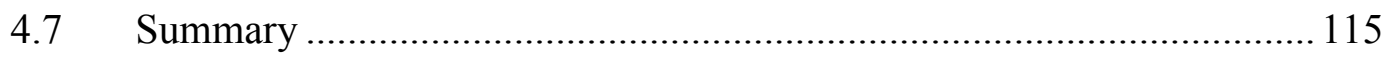

CHAPTER 5.|PRACTICAL MULTIPLE-FANS IN REAL COAL MINES ................. 122

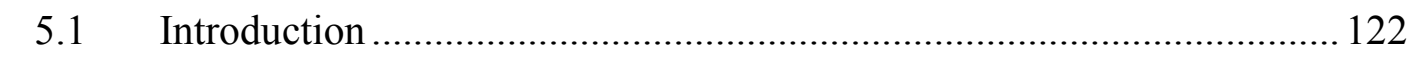

$5.2 \quad$ Ventilation Performance in Mine A .................................................. 127

$5.3 \quad$ Ventilation Performance in Mine B ............................................... 132

$5.4 \quad$ Ventilation Performance in Mine C .................................................. 138

$5.5 \quad$ Relation Between Airflow and Resistance Factor in Real Coal Mine

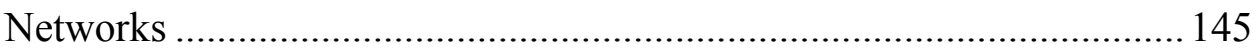

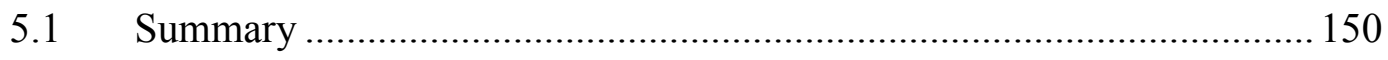

CHAPTER 6.|CONCLUSION AND CONTRIBUTIONS ....................................... 152

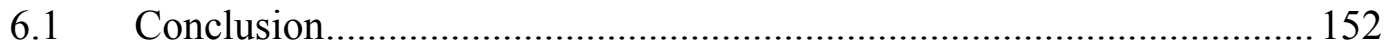

6.2 Contribution .................................................................................. 155

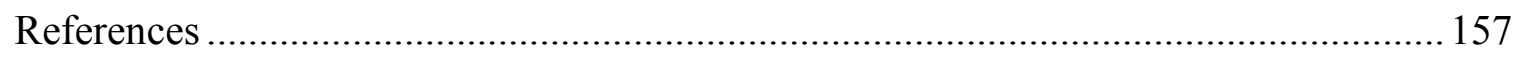

APPENDIX A. SCHEMATIC LINE VENTILATION DIAGRAMS IN POCKET

A.1 Schematic line vantilation diagram for mine A IN POCKET

A. 2 Schematic line vantilation diagram for mine B IN POCKET 


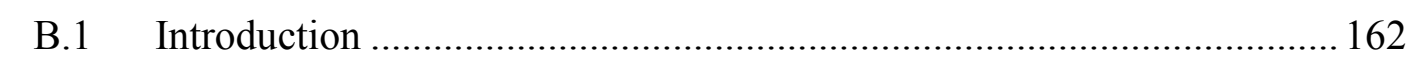

B.2 The MATLAB.m file hc.m........................................................... 163

B.2.1 The MATLAB.m file mesh.m ................................................... 166

B.2.2 The MATLAB.m file resis.m ..................................................... 166

B.2.3 The MATLAB.m file ind.m .................................................... 166

B.2.4 The MATLAB.m file eval_f.m...................................................167

B.2.5 The MATLAB.m file t1.m ....................................................... 167

B.2.6 The MATLAB.m file t2.m ................................................... 169

B.2.7 The MATLAB.m file try1.m ....................................................... 170

B.2.8 The MATLAB.m file try2.m................................................. 172

B.2.9 The MATLAB.m file try3.m................................................... 174

B.3 The MATLAB.m file newton.m....................................................... 176

B.3.1 The MATLAB.m file gauss.m.................................................. 179

B.3.2 The MATLAB.m file jacob.m........................................................ 180 


\section{LIST OF TABLES}

$\underline{\text { Table \# }} \quad \underline{\text { Page }}$

3.1 Operating points obtained from the different subsystems 56

3.2 Standard deviation and correlation coefficient for some statistical models

68

5.1 Main mine fan's quantities and total pressures for mine A

5.2 Main mine fan's quantities and total pressures for mine B

133

5.3 Main mine fan's quantities and total pressures for mine C

5.4

Resistance for the subsystems

146 


\section{LIST OF FIGURES}

Figure \# $\quad \underline{\text { Page }}$

1.1 Fan and mine characteristic curves in a single fan system 4

1.2 Subsystem and fan characteristic curves in a three dimensional space (Wang, 1992)

2.1 An example network with $\mathrm{n}_{\mathrm{b}}=10$ and $\mathrm{n}_{\mathrm{n}}=6 \quad 13$

2.2 Illustrative figure for the Hardy Cross method 22

$2.3 \Delta$-circuit Y-circuit, Kumazawa (1942) 27

2.4 Geometric interpretation of Newton's method 28

3.1 Switching parameter between variables $\mathrm{x}$ and $\mathrm{y} \quad 43$

3.2 Major cutset, Wang (1983) 45

3.3 Flow chart of Hardy Cross with the switching-parameter algorithm 48

3.4 The layout and the resistance factors for the preliminary example network 57

3.5 Fan characteristic curves A, B and C 58

$\begin{array}{lll}3.6 & \text { Subsystem characteristic curve } P_{1} & 60\end{array}$

3.7 Subsystem characteristic curve $\mathrm{P}_{2}$

$\begin{array}{lll}3.8 & \text { Subsystem characteristic curve } \mathrm{P}_{3} & 62\end{array}$

$\begin{array}{lll}3.9 & \text { Subsystem characteristic curve } \mathrm{P}_{4} & 63\end{array}$

3.10 Subsystem characteristic curves $U_{112}$ and $U_{312}$

3.11 Subsystem characteristic curves $U_{113}$ and $U_{313}$

3.12 Subsystem characteristic curves $U_{223}$ and $U_{323}$

3.13 Subsystem characteristic curves $U_{212}$ and $U_{312}$

3.14 Subsystem characteristic curves $U_{134}$ and $U_{434}$ 
Figure \# $\quad$ Page

3.15 Subsystem characteristic curves $\mathrm{U}_{224}$ and $\mathrm{U}_{324}$

4.1 Effect of changing the resistance of fan branch No. 1 on the shape of its subsystem characteristic curve

4.2 Effect of changing the resistance of fan branch No. 1 on the shape of subsystem No. 2 characteristic curve

4.3 Effect of changing the resistance of fan branch No. 1 on the shape of subsystem No. 3 characteristic curve

4.4 Effect of changing the resistance of fan branch No. 1 on the shape of subsystem No. 4 characteristic curve

4.5 Effect of changing the resistance of regular branch No. 9 on the shape of subsystem No. 1 characteristic curve

4.6 Effect of changing the resistance of regular branch No. 9 on the shape of subsystem No. 2 characteristic curve

4.7 Effect of changing the resistance of regular branch No. 9 on the shape of subsystem No. 3 characteristic curve

4.8 Effect of changing the resistance of regular branch No. 9 on the shape of subsystem No. 4 characteristic curve

4.9 The topology of the 8 meshes, 9 nodes and 16 branches network 85

4.10 The topology of the 16 meshes, 14 nodes and 29 branches network 86

4.11 Effect of changing the topology of the network on the shape of subsystem No. 1 characteristic curve

4.12 Effect of changing the topology of the network on the shape of subsystem No. 2 characteristic curve

4.13 Effect of changing the topology of the network on the shape of subsystem No. 3 characteristic curve

4.14 Effect of changing the topology of the network on the shape of subsystem No. 4 characteristic curve 
4.16 The layout of a network contains 10 branches, 6 nodes and one fan installed in branch No. 1

4.17 The layout of a network contains 10 branches, 6 nodes and three fans installed in branches No. 1 and 4

4.18 The layout of a network contains 10 branches, 6 nodes and two fans installed in branches No. 1, 3 and 4

4.19 The layout of a network contains 10 branches, 6 nodes and two fans installed in branches No. 1, 2, 3 and 4

4.20 The effect of changing No. of fans in the system on the shape of subsystem No. 1 characteristic curve

4.21 The effect of changing No. of fans in the system on the shape of subsystem No. 4 characteristic curve

4.22 The effect of changing No. of fans in the system on the shape of subsystem No. 3 characteristic curve

4.23 The effect of changing No. of fans in the system on the shape of subsystem No. 2 characteristic curve

4.24 Shape of subsystem No. 1 characteristic curve in a 4 axial flow fans system

4.25 Shape of subsystem No. 1 characteristic curve in a 3 axial and

1 centrifugal flow fans system

4.26 Shape of subsystem No. 1 characteristic curve in a 2 axial and 2 centrifugal flow fans system

4.27 Shape of subsystem No. 1 characteristic curve in a 1 axial and 3 centrifugal flow fans system

4.28 Shape of subsystem No. 1 characteristic curve in a 4 centrifugal fans system

4.29 Shape of subsystem No. 2 characteristic curve in a 4 axial flow fans system

4.30 Shape of subsystem No. 2 characteristic curve in a 3 axial and 1 centrifugal flow fans system 
4.31 Shape of subsystem No. 2 characteristic curve in a 2 axial and 2 centrifugal flow fans system

4.32 Shape of subsystem No. 2 characteristic curve in a 1 axial and 3 centrifugal flow fans system

4.33 Shape of subsystem No. 2 characteristic curve in a 4 centrifugal fans system

4.34 Shape of subsystem No. 3 characteristic curve in a 4 axial flow fans system

4.35 Shape of subsystem No. 3 characteristic curve in a 3 axial and 1 centrifugal flow fans system

4.36 Shape of subsystem No. 3 characteristic curve in a 2 axial and 2 centrifugal flow fans system

4.37 Shape of subsystem No. 3 characteristic curve in a 1 axial and 3 centrifugal flow fans system

4.38 Shape of subsystem No. 3 characteristic curve in a 4 centrifugal fans system

4.39 Shape of subsystem No. 4 characteristic curve in a 4 axial flow fans system

4.40 Shape of subsystem No. 4 characteristic curve in a 3 axial and 1 centrifugal flow fans system

4.41 Shape of subsystem No. 4 characteristic curve in a 2 axial and 2 centrifugal flow fans system

4.42 Shape of subsystem No. 4 characteristic curve in a 1 axial and 3 centrifugal flow fans system

4.43 Shape of subsystem No. 4 characteristic curve in a 4 centrifugal fans system

4.45 Effect of changing the speed of fan No. 3 on the shape of 
4.46 Effect of changing the speed of fan No. 3 on the shape of Subsystem No. 2

4.47 Effect of changing the speed of fan No. 3 on the shape of Subsystem No. 4

5.1 Fan performance curve for JoyM120-65 at different blade angles

5.2 Fan performance curve for Jeffrey 8HUA96 at different blade angles

5.3 Fan performance curve for Zurn/Clarge 153 AFM-5350 SWSI Centrifugal at different blade angles

5.4 Subsystem No. 1 characteristic curve (Mine A)

5.5 Subsystem No. 2 characteristic curve (Mine A)

5.6 Subsystem No. 3 characteristic curve (Mine A)

5.7 Subsystem No. 1 characteristic curve (Mine B)

5.8 Subsystem No. 2 characteristic curve (Mine B)

5.9 Subsystem No. 3 characteristic curve (Mine B)

5.10 Subsystem No. 4 characteristic curve (Mine B)

5.11 Subsystem No. 1 characteristic curve (Mine C)

5.12 Subsystem No. 2 characteristic curve (Mine C)

5.13 Subsystem No. 3 characteristic curve (Mine C)

5.14 Subsystem No. 4 characteristic curve (Mine C)

5.15 Subsystem No. 5 characteristic curve (Mine C)

5.16 The relation between air quantity and resistance factor in mine A

5.17 The relation between air quantity and resistance factor in mine B

5.18 The relation between air quantity and resistance factor in mine C 


\section{NOMENCLATURE}

Symbol

$\mathrm{A}_{\mathrm{a}}$

$a_{i j}$

B

$b_{i j}$

C

$\mathrm{c}_{\mathrm{ij}}$

delta-q

F

$F_{i}(x)$

$F_{i}$

$\mathrm{H}_{\mathrm{f}}$

$\mathrm{H}_{\mathrm{i}}$

$\mathrm{H}_{\mathrm{nj}}$

$h_{f_{j}}$

$\mathrm{h}_{\mathrm{i}}$

$\mathrm{h}_{\mathrm{q}}$

$h_{R_{j}}$

it_no

$\mathrm{k}$

$\mathrm{L}_{\mathrm{k}}$

M $\underline{\text { Description }}$

$\underline{\text { Units }}$

Incident matrix

Element of the incident matrix

Fundamental mesh matrix

Element of the fundamental mesh matrix

Fundamental Cutset matrix

Element of the Fundamental cutset matrix

Correction value applied to the flow distribution

$\mathrm{m}^{3} / \mathrm{sec}$

Number of fans in the system

Function $\mathrm{i}$ in $(\mathrm{x})$

Correction value of mesh I

$\mathrm{m}^{3} / \mathrm{sec}$

Pressure loss due to friction

$\mathrm{Pa}$

Pressure drop through the system i

$\mathrm{Pa}$

Natural ventilation pressure

$\mathrm{Pa}$

Fan head pressure

$\mathrm{Pa}$

Pressure loss through branch i

$\mathrm{Pa}$

Step size

Regulator head pressure

$\mathrm{Pa}$

Maximum iteration number

Subsystem number

The solution curve for subsystem $\mathrm{k}$

Number of fundamental meshes in the network 


\begin{tabular}{|c|c|c|}
\hline Symbol & Description & Units \\
\hline max-delta & Tolerance value & - \\
\hline $\mathrm{N}$ & Maximum number of points in the solution curve & - \\
\hline \multirow[t]{2}{*}{$\mathrm{n}$} & Number of equations in the system, and a solution & \\
\hline & point number & - \\
\hline $\mathrm{n}_{\mathrm{b}}$ & Number of branches in the network & - \\
\hline $\mathrm{n}_{\mathrm{c}}$ & Number of the fundamental cutsets in the network & - \\
\hline $\mathrm{n}_{\mathrm{n}}$ & Number of nodes in the network & - \\
\hline$P_{i}$ & Pressure drops through a subsystem i & $\mathrm{Pa}$ \\
\hline$P_{k}$ & The projection of the solution curve $\mathrm{L}_{\mathrm{k}}$ on the $\mathrm{q}_{\mathrm{k}}-\mathrm{p}_{\mathrm{k}}$ plane & - \\
\hline $\mathrm{p}$ & Pressure & $\mathrm{Pa}$ \\
\hline Q & Total airflow through the system & $\mathrm{m}^{3} / \mathrm{sec}$ \\
\hline $\mathrm{Q}_{\mathrm{i}}$ & Total airflow through subsystem i & $\mathrm{m}^{3} / \mathrm{sec}$ \\
\hline $\mathrm{q}_{\mathrm{a}}$ & Initial guess of the flow distribution & $\mathrm{m}^{3} / \mathrm{sec}$ \\
\hline $\mathrm{q}_{1}, \ldots, \mathrm{q}_{\mathrm{M}}$ & The independent airflow branches & $\mathrm{m}^{3} / \mathrm{sec}$ \\
\hline$q_{i}$ & Airflow through the airway (i) & $\mathrm{m}^{3} / \mathrm{sec}$ \\
\hline \multirow[t]{2}{*}{ q_in } & Initial values of the air quantities flow through the & \\
\hline & major cutset & $\mathrm{m}^{3} / \mathrm{sec}$ \\
\hline q index & Air quantity flow through the independent branch & $\mathrm{m}^{3} / \mathrm{sec}$ \\
\hline $\mathrm{R}$ & Mine resistance factor & N.s ${ }^{2} / \mathrm{m}^{8}$ \\
\hline $\mathrm{R}_{\mathrm{eq}}$ & The system equivalent resistance & $\mathrm{N} . \mathrm{s}^{2} / \mathrm{m}^{8}$ \\
\hline $\mathrm{R}_{\mathrm{i}}$ & Subsystem i resistance factor & N.s $\mathrm{s}^{2} / \mathrm{m}^{8}$ \\
\hline $\mathrm{r}_{\mathrm{i}}$ & Branch i resistance factor & N.s $\mathrm{s}^{2} / \mathrm{m}^{8}$ \\
\hline
\end{tabular}




$\begin{array}{lll}\text { Symbol } & \text { Description } & \text { Units } \\ \mathrm{t}_{\mathrm{i}} & \text { Fan i characteristic curve } & \text { Pa } \\ \mathrm{t}_{\mathrm{i}}\left(\mathrm{q}_{\mathrm{i}}\right) & \text { pressure delivered from a fan i at a quantity } \mathrm{q}_{\mathrm{i}} & \text { - } \\ \mathrm{U}_{\mathrm{kij}} & \text { The projection of the solution curve } \mathrm{L}_{\mathrm{k}} \text { on the } \mathrm{q}_{\mathrm{i}}-\mathrm{q}_{\mathrm{j}} \text { plane } & \\ \mathrm{Y} & \text { Three-terminal circuit } & \text { Watt } \\ \mathrm{Z}_{\mathrm{i}} & \text { Power consumed through a subsystem (i) } & \\ \Delta & \text { Difference, Delta (two-leg)-circuit, and the approximate } & \\ \alpha_{\mathrm{j}} & \text { correction value } & \text { - } \\ \beta_{\mathrm{j}} & 1^{\text {st }} \text { coefficient in the fan }(\mathrm{j}) \text { equation } & \text { - } \\ \gamma_{\mathrm{j}} & 2^{\text {nd }} \text { coefficient in the fan }(\mathrm{j}) \text { equation } & \text { - }\end{array}$




\section{CHAPTER 1 INTRODUCTION AND PROBLEM STATEMENT}

\subsection{Introduction}

A ventilation system is composed of a fan or fans and a set of connected mine openings. A single-fan network can be easily divided into two main ventilation components, the fan and the connected mine openings (or branches). By dividing the system in this fashion two characteristic curves may be drawn, the fan curve and mine characteristic curve. The mine characteristic curve, according to Atkinson's equation, in a single fan system is a strictly increasing function at a given specific air weight and a constant fan speed. However, this relationship holds true for a ventilation system with a single fan, but generally cannot be directly applied to multiple-fan networks because of the multiple pressures and air quantities that are associated with the different fans in the network and their interaction with each other (Wang 1984b). Accordingly, each fan in a multiple-fan system has its own mine characteristic curve or subsystem curve associated with it. Many researchers have studied the interaction of fans in multiple-fan systems. The effect is seen as an irregularity in the shape of each fan's subsystem curve and in the resulting number of operating points in the system. This phenomenon may occur with many different axial flow fans, especially if one or more of those fans is operating outside of its proper range. As a result mine fans may operate frequently between these operating points which appears as a considerable throb and pulsation accompanied with noise, rumbling and high vibration (Harrison and Kutay, 1986). This phenomenon may cause a damage of one or more of the fans in the system which considerably effect on the stability of the whole system. 


\subsection{Problem Statement}

One fan classification system is defined according to the direction of the flow through the impeller of axial-flow, radial-flow, mixed-flow, and cross-flow fans (Jorgenson 1983). The two types of fans most commonly used in mines are axial and radial flow fans. In an axial fan air passes through the fan housing along flow paths that are aligned with the rotational axis of the vane impeller without changing the airflow direction. Radial flow fans, more commonly called centrifugal fans, resemble paddle wheels. Air enters near the center of the wheel and must make a right-angle turn, and then moves radially outward by centrifugal action between the blades of the rotating impeller (McPherson 1993). The air, now moving along the housing, continues to change direction until it reaches the exit.

In multiple-fan systems, the shape of subsystem characteristic curves dependent upon the type of fan or fans inside this subsystem. If the subsystem has only centrifugal fans, the shape of the subsystem characteristic curve is still a strictly increasing function similar to the single fan system, but it is possible that it may not pass through the point of origin. On the other hand, if the subsystem contains only axial flow fans, previous studies of multiple-fan ventilation systems indicate that the mine characteristic curve has sharp turning points and does not follow nor is it strictly increasing. Moreover, each fan in the system has its own subsystem characteristic curve.

The physical equilibrium between the mine and the fan represents the operating point of the system. This equilibrium occurs at the intersection of the fan and subsystem 
curves, and is called the operating point. There is only one operating point in either single or multiple-centrifugal fan systems. However, the mine (or subsystem) and fan characteristic curves in either single or multiple axial-flow fans systems may intersect more than one, resulting in more than one operating point (Figure 1.1). In a single-fan system, unstable conditions are usually avoided by restricting fan operation to its stable operating range by changing either its speed or its blade angle.

Series-parallel connections in multiple-fan systems can be graphically represented in a quantity-pressure, q-p, coordinate system. In this case, the number of operating points can be obtained directly from the graph. Moreover, they can be calculated numerically by applying any mathematical techniques that solve $\mathrm{M}$ nonlinear equations in $\mathrm{M}+1$ unknowns. These methods use different initial guesses for the solution, and then are solved by iteration until they converge at all operating points within the system. However, in complex multiple-axial fan systems, it is not possible to plot the combined fan characteristic curve. Neither the relation between pressure and airflow is clear, nor is the number of operating points known. Conventional numerical techniques, although capable of finding some operating points by using different initial guesses, are not guaranteed to converge at all possible operating points within the system. Therefore a new numerical approach has to be developed to draw the subsystem characteristic curve for each fan in the system, and then determine all possible operating points. 


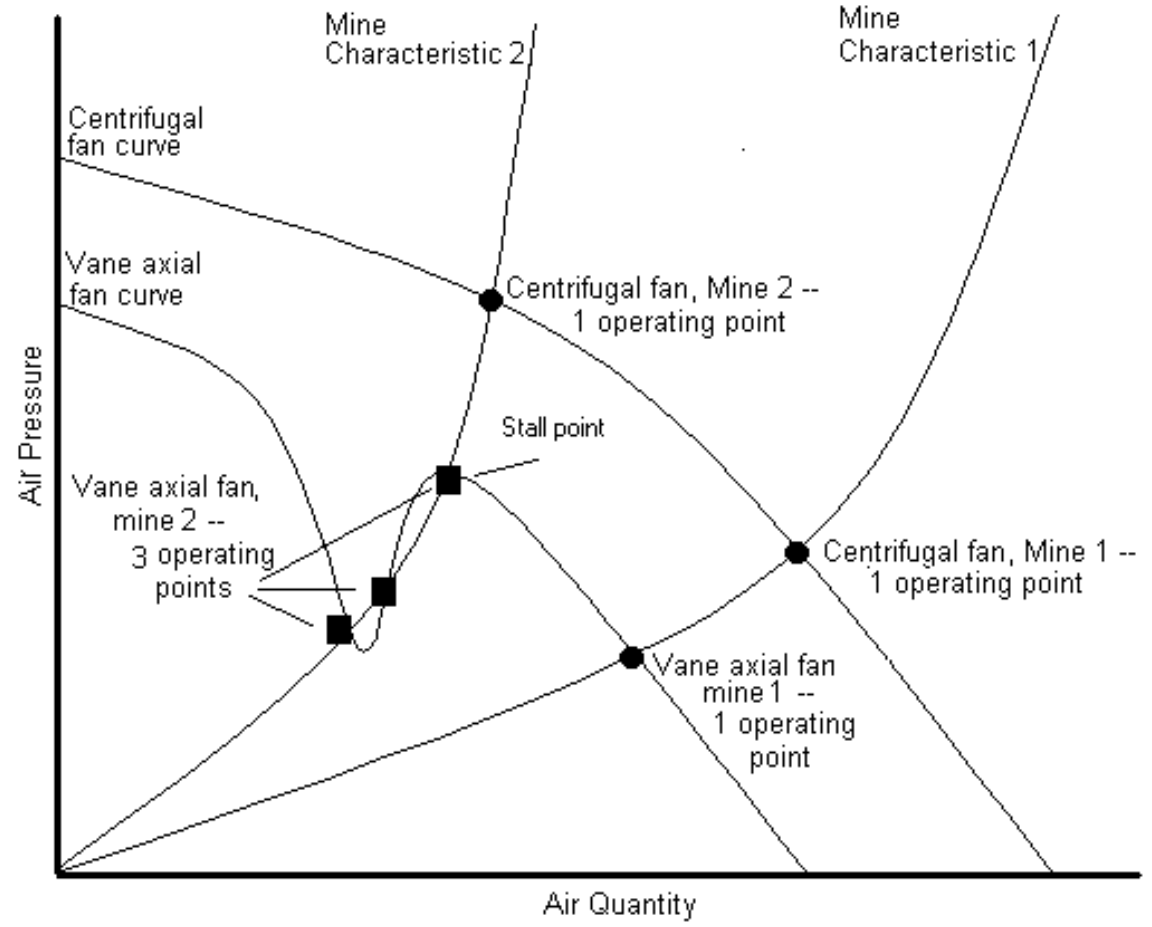

Figure 1.1 -Fan and mine characteristic curves in a single fan system 


\subsection{Background}

In multiple-fan systems, an individual fan influences the performance of the whole system, including the other fans. Professor Y.J Wang and his students in West Virginia University have investigated multiple-fan ventilation systems extensively. Their purpose was to study the shape of mine characteristic curves, examine the conditions of instabilities, and then formulate equations defining the combined system.

Multiple fan system is a steady state analysis. However, the system resistance is not constant unless the quantity ratios of the subsystems are held constant (Wang 1984b). By considering this new approach to the system resistance, the conventional concept of single-fan system mine characteristic curve may be directly extended to a particular fan in a multiple-fan system. However rather than a single mine characteristic curve $\mathrm{P}$ in the q-p plane for the entire system of a single-fan system, there are F subsystem characteristic curves in the corresponding $\mathrm{q}_{\mathrm{f}}-\mathrm{p}_{\mathrm{f}}$ planes of a multiple fan system, one for each fan. Fans inside any subsystem could work as variable resistances. The system operating characteristics with respect to one of the fans can be delineated through a subsystem characteristic curve in a multiple-dimensional space (Figure 1.2, Wang 1992). In a single fan system the number of unknowns is equal to the number of meshes in a system. However, in the multiple fan system, the number of unknowns is equal to the number of meshes, but the pressure consumed throughout the subsystem is also unknown. Thus the number of unknowns in the multiple fan system is one more than the number of equations (M) that mathematically represent the system. Consequently, the representation of the subsystem is difficult (or impossible) to visualize if the number of meshes in the 
network is more than two, since the dimensional space of the solution is $\mathrm{M}+1$. This concept is examined in detail in chapter 3.

The classic Hardy Cross method (Cross, 1936) cannot be used to solve the set of equations representing the multiple-fan system because it does not contain a single term from the fan characteristic curve representing the pressure gain from the fan. Even the improved version of the Hardy Cross method by Scott and Hinsley (1951), which considers the characteristic of a single fan, is not capable of tracing the subsystem characteristic curve in multiple directions.

The standard Newton-Raphson numerical method (Ortega and Rheinboldt, 1970) cannot be used, since it converges only if the initial guess is close to the actual solution. There is no known algorithm to choose an appropriate set of initial guesses. While the Newton-Raphson method, combined with the switching parameter technique (Wang and Reddy, 1992) succeeds in obtaining all operating points for some problems, it still requires more than one initial guess for many problems and does not guarantee that all solutions have been found.

The Quasi-Newton algorithm compound with a switching-parameter (Lin, 1995) has two advantages compared to the conventional and improved Newton-Raphson methods. The first advantage is that the number of computation iteration is greatly reduced. The second is the subsystem characteristic curve can be plotted simultaneously with its reference fan characteristic curve to graphically check the number of all possible 
operating points. Although the Quasi-Newton algorithm with switching-parameter is much more effective and efficient, it still needs more than one guess to trace and draw the subsystem characteristic curve. Thus, no mathematical model can trace the solution curve for the multiple fan system and find all operating points with one initial guess.

\subsection{Objectives}

The objectives of this study are to:

- Develop numerical models that can effectively solve $\mathrm{M}$ nonlinear equations with $(\mathrm{M}+1)$ variables, draw the subsystem characteristic curve, and obtain all the operating points in the multiple fan system by using only a single initial guess for the solution.

- Investigate the various parameters that affect the shape of the solution curve and the shape of the subsystem characteristic curve of each fan, use this understanding to improve its shape, reduce the number of turning points and reduce the number of operating points in the system.

- Study the relation between pressure and airflow consumed in each subsystem and examine the validity of applying Atkinson equation on multiple-fan system.

- Apply the numerical model to data from operating multiple-fan coal mines to validate the model. 


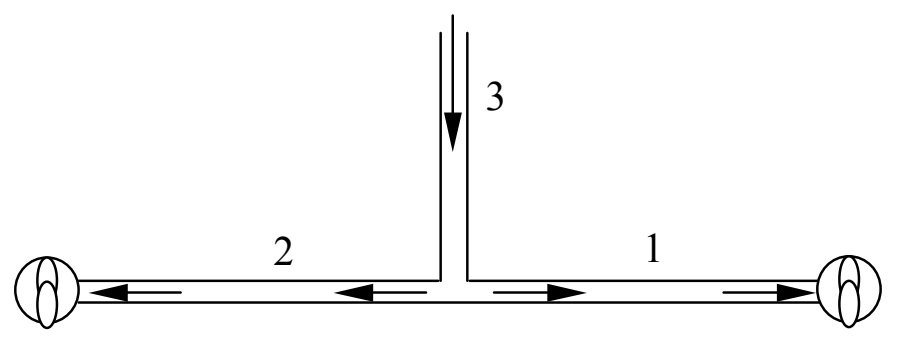

Simplified 2-fan system



Figure 1.2 -Subsystem and fan characteristic curves in a three dimensional space. (after Wang, 1992) 


\section{CHAPTER 2 \\ LITERATURE REVIEW}

\subsection{Introduction}

Airways in mine ventilation circuits can be arranged in three basic configurations: series, parallel or complex. In series circuits, airways are connected end to end so that the total pressure drop across the connection is the sum of pressure drops across each airway while the same air quantity flows through each of them. In parallel airways, the pressure drop across the entire network is the same, while quantities are distributed among the airways according to their respective resistances. Using calculated equivalent resistances is the most primary method of analyzing ventilation networks (McPherson, 1993, p. 215). The equivalent resistance $R_{\mathrm{eq}}$ can be calculated for series connections by adding the values of resistances $r_{i}$ as follows:

$$
\begin{aligned}
& R_{e q} * \boldsymbol{Q}^{2}=\sum_{i=1}^{n} r_{i} \boldsymbol{Q}^{2} \\
& R_{e q}=\sum_{i=1}^{n} r_{i}
\end{aligned}
$$

The equivalent resistance of parallel circuits can be estimated by:

$$
\begin{aligned}
& \frac{\Delta p}{\sqrt{R_{e q}}}=\sum_{i=1}^{n} \frac{\Delta p}{\sqrt{r_{i}}} \\
& \frac{1}{\sqrt{R_{e q}}}=\sum_{i=1}^{n} \frac{1}{\sqrt{r_{i}}}
\end{aligned}
$$


A characteristic feature of a complex circuit is the presence of one or more diagonal connections. Consequently the network cannot be reduced to an equivalent airway by using equations 2.1 and 2.2. Along these diagonals depending on the pressure in the airways, air may flow in either directions, or not at all (Vutukuri and Lama, 1986). Complex circuits can be reduced to workable models by using some basic techniques. By assuming that the air in mine airways is uncompressible and that it follows Atkinson's equation, the solution of complex networks can be achieved by combining Atkinson's equation and Kirchhoff's laws. In order to apply these laws consistently some basic network terminology has been defined, including: (Bunt et al., 1960).

- A junction or node is a point where two or more airways meet.

- A branch is a segment of an airway connecting two junctions.

- A mesh is a closed loop or path traversed by following airways within the network.

\subsection{Basic Laws Applied in the Analysis of Mine Ventilation Systems}

An analogy may be drawn between the square law of mine ventilation or Atkinson's equation (equation 2.3) and Ohm's law. Airflow, head losses and airway resistance in mine ventilation circuits are equivalent to current, voltage and electric resistance in electric circuits, respectively. Thus by taking into consideration the second order of the square law of mine ventilation, it is possible to apply electric laws to mine networks. In this fashion, V=IR becomes

$$
H_{f}=R Q^{2}
$$


Kirchhoff's current law, an application of conservation of mass, states that the total quantity of air flowing towards a junction is equal to the total quantity leaving (equation 2.4). Kirchhoff's voltage law, an application of conservation of energy, states that the algebraic sum of all pressure drops in a closed loop must equal zero (equation 2.5) (Wang, 1982a, pp 167-195).

$$
\begin{aligned}
& \sum_{i=1}^{j} q_{i}=0 \\
& \sum_{i=1}^{j} r_{i}\left|q_{i}\right| q_{i}+p_{i}-T_{i}-H_{n}=0
\end{aligned}
$$

$\left|q_{i}\right| q_{i}$ is used instead of $q_{i}^{2}$ in order to preserve negative values if present, an indicator of direction. Using equations 2.3, 2.4 and 2.5, the mine ventilation system can be defined in terms of a network. When the number of unknown variables is equal to the number of network equations, the system is a conventional network problem and has a unique solution. Otherwise, when the number of variables exceeds the number of network equations, the system becomes an optimization problem and has many solutions.

A mine ventilation network represents a real world mine ventilation system. A collection of nodes and branches is called a linear graph of the real world mine ventilation system. Therefore, the topological properties of a linear graph from the graph theory can be directly applied to a mine ventilation-network problem.

A spanning tree, or a tree of a network, is defined as a connected sub-network that contains all the nodes of a network but does not contain any meshes. Branches that construct the tree are called tree branches while the remaining branches in the network 
are called chords (Wang, 1982 a, pp 483-516). Figure 2.1 shows the tree and chord branches as single and double lines, respectively. An incident matrix $\left(\mathrm{A}_{\mathrm{a}}\right)$ of a network is an $n_{n} * n_{b}$ matrix, where $n_{n}$ and $n_{b}$ are the number of nodes and the number of branches in the network, respectively. Each row of this matrix represents a node and each column represents a branch in the network. Its elements $\left[\mathrm{a}_{\mathrm{ij}}\right]$ are defined as follows:

$\mathrm{a}_{\mathrm{ij}}=1$ if branch $\mathrm{j}$ is incident at node $\mathrm{i}$ and is directed away from node $\mathrm{i}$.

$a_{i j}=-1$ if branch $\mathrm{j}$ is incident at node $\mathrm{i}$ and is directed toward node $\mathrm{i}$.

$\mathrm{a}_{\mathrm{ij}}=0$ if branch $\mathrm{j}$ is not incident at node $\mathrm{i}$.

Consequently, each column will have just two non-zero elements, 1 and -1 .

A fundamental matrix (B) of a network is an $\mathrm{M} * \mathrm{n}_{\mathrm{b}}$ matrix, where $\mathrm{M}$ is the number of fundamental meshes with respect to a tree in the network. Each row of the fundamental matrix represents a fundamental mesh in the network. As a result, every fundamental mesh contains only one chord, a unique chain of the tree branches connecting two endpoints of that chord, and each chord is contained in just one mesh. Elements of the fundamental mesh matrix $\left[b_{i j}\right]$ are defined as follows:

$\mathrm{b}_{\mathrm{ij}}=1$ if branch $\mathrm{j}$ is contained in mesh $\mathrm{i}$ and has the same mesh direction. $b_{i j}=-1$ if branch $\mathrm{j}$ is contained in mesh $\mathrm{i}$ and has the opposite mesh direction. $\mathrm{b}_{\mathrm{ij}}=0$ if branch $\mathrm{j}$ is not contained in mesh $\mathrm{i}$.

As an illustrative example, the number of tree branches in the network shown in Figure 2.1 equal to $\left(n_{n}-1\right)=6-1=5$ and number of chords which equals the number of fundamental meshes is $(M)=\left(n_{b}-n_{n}+1\right)=10-6+1=5$. The incident matrix of the network can be represented as: 


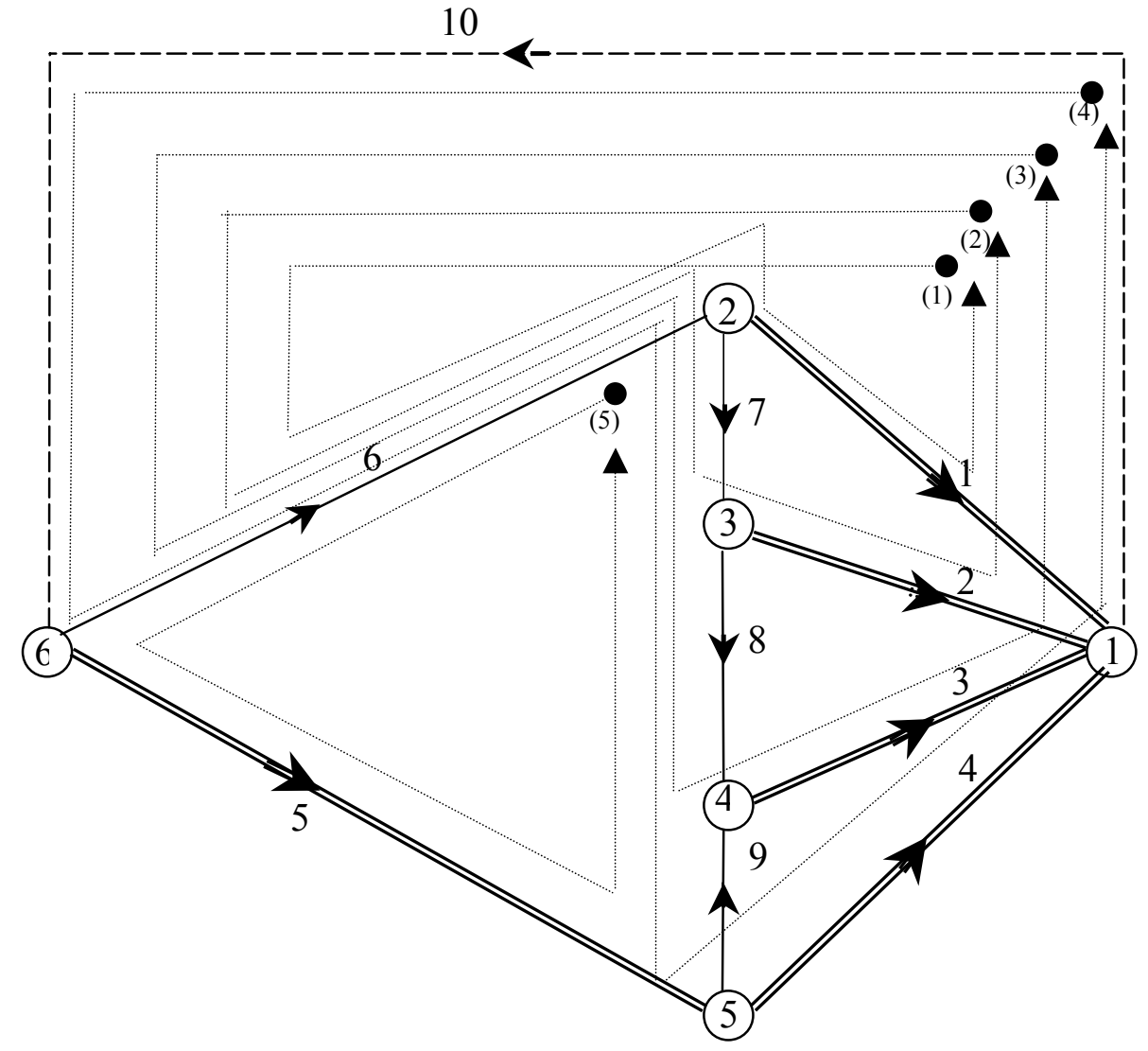

Figure 2.1 An example network with $n_{b}=10$ and $n_{n}=6$. 


$$
A_{a}=\begin{array}{r}
1 \\
3 \\
4 \\
4 \\
5 \\
6 \\
6
\end{array}\left[\begin{array}{cccccccccc}
-1 & -1 & -1 & -1 & 0 & 0 & 0 & 0 & 0 & 1 \\
1 & 0 & 0 & 0 & 0 & -1 & 1 & 0 & 0 & 0 \\
0 & 1 & 0 & 0 & 0 & 0 & -1 & 1 & 0 & 0 \\
0 & 0 & 1 & 0 & 0 & 0 & 0 & -1 & -1 & 0 \\
0 & 0 & 0 & 1 & -1 & 0 & 0 & 0 & 1 & 0 \\
0 & 0 & 0 & 0 & 1 & 1 & 0 & 0 & 0 & -1
\end{array}\right]
$$

By choosing a tree consisting of branches $6,7,8,9$ and 10 choosing the direction of each fundamental mesh to agree with that of the defining chord, (shown as double lines and dotted arrows respectively in Figure 2.1), then the fundamental meshes with respect to the tree can be expressed in terms of branches number as follows:

$\begin{array}{lllllll}\text { Mesh 1: } & 1 & 6 & 10 & & & \\ \text { Mesh 2: } & 2 & 6 & 7 & 10 & & \\ \text { Mesh 3: } & 3 & 6 & 7 & 8 & 10 & \\ \text { Mesh 4: } & 4 & 6 & 7 & 8 & -9 & 10 \\ \text { Mesh 5: } & 5 & -6 & -7 & -8 & 9 & \end{array}$

The corresponding fundamental mesh matrix is given by

$$
\boldsymbol{B}=3\left[\begin{array}{cccccccccc}
\mathbf{1} & \mathbf{2} & \mathbf{3} & \mathbf{4} & \mathbf{5} & \mathbf{6} & \mathbf{7} & \mathbf{8} & \mathbf{9} & \mathbf{1 0} \\
1 & 0 & 0 & 0 & 0 & 1 & 0 & 0 & 0 & 1 \\
0 & 1 & 0 & 0 & 0 & 1 & 1 & 0 & 0 & 1 \\
0 & 0 & 1 & 0 & 0 & 1 & 1 & 1 & 0 & 1 \\
0 & 0 & 0 & 1 & 0 & 1 & 1 & 1 & -1 & 1 \\
0 & 0 & 0 & 0 & 1 & -1 & -1 & -1 & 1 & 0
\end{array}\right]
$$




\subsection{Single Fan Systems}

In a single fan system, the equilibrium point between fan and mine can be determined as the operating point of the system. At this point, the head generated by the fan (pressure gain) is equal to the mine head (pressure loss). The head and quantity at the operating point can be read directly from the intersection of fan and mine characteristic curves, as shown in Figure 1.1. A mine Characteristic curve in a single fan system is a strictly increasing function and can be expressed analytically in the form of Atkinson's Equation (2.3).

Single centrifugal fan systems have only one operating point (see note section 1.1). However, in a single axial flow fan system, more than one operating point may exist if the fan operates in the zone of instability, to the left of the stall point, (Figure 1.1). Multiple operating points frequently may be avoided by changing the blade-setting angle, and/or fan speed. Changing the angle of pitch of the blades is the most frequent method used to improve the performance of an axial flow fan (Wang, 1982, p. 282). The use of fans with variable pitch fan blades has introduced greater flexibility in the operation of axial flow fans (Willis, 1961, p. 260). There is no mathematical relation that will define the effect of changing the pitch angle of the blades; the performance and efficiency must be determined by experimental data provided by fan manufacturers. Morris and Hinsley (1951) proved that air quantity varies proportionally and head varies directly to a fractional power in relation to a pitch angle. There are clear mathematical relations between fan performance, size and speed, as indicated in following table (McPherson, 1993, p. 345). 


\begin{tabular}{lcc}
\hline Variable, $\mathrm{n}$ & Variable, $\mathrm{d}$ & Variable, $\rho$ \\
\hline $\mathrm{P} \infty \mathrm{n}^{2}$ & $\mathrm{p} \infty \mathrm{d}^{2}$ & $\mathrm{p} \infty \rho$ \\
$\mathrm{Q} \infty \mathrm{n}$ & $\mathrm{Q} \infty \mathrm{d}^{3}$ & $\mathrm{Q}$ fixed \\
$\mathrm{P}_{\text {ow }} \infty \mathrm{n}^{3}$ & $\mathrm{P}_{\text {ow }} \infty \mathrm{d}^{5}$ & $\mathrm{P}_{\text {ow }} \infty \rho$ \\
\hline
\end{tabular}

Where $\mathrm{P}$ is the pressure, $\mathrm{n}$ is the speed in rpm, $\mathrm{d}$ is the fan diameter and $\mathrm{P}_{\mathrm{ow}}$ is the fan power.

\subsection{Multiple Fan Systems}

Multiple-fan ventilation systems are widely used in the US to provide an adequate air supply and insure appropriate mine air quantity and quality control. The system resistance offered to each individual fan becomes a function not only of the network geometry, but also of the location and operating characteristics of each of the other fans in the system. Consequently, each fan in the system has its own mine characteristic curve or subsystem characteristic curve. Fans may be connected in series, in parallel or in combinations. When fans are connected in series, each fan contributes a part of the total network pressure and handles the total airflow of the circuit. In practice, a series arrangement may be applied in the later stages of the life of a mine when mine resistance has become high and the mine characteristic has become steep. When fans are connected in parallel, each fan contributes a part of overall airflow and generates a pressure equal to the head loss of the entire circuit. Mine ventilation networks, with fans connected in series or parallel, can be solved graphically by applying the multiple series-parallel reduction (Wang, 1989) 
Complex multiple-fan networks may be represented by applying the principle of mine characteristic curves and using a subsystem approach. Each subsystem (for each fan) has its own solution curve. Therefore, the subsystem curve is the relation between the airflow through it and the pressure drop across it. Each of these subsystems contributes a part of the total pressure head and total air quantity of the system. The solution curve is generated by replacing the reference fan with a hypothetical pressure source or, alternatively, by adding a hypothetical pressure source without removing a fan. However, instead of the single mine characteristic curve P in the q-p plane, there are F characteristic curves $P_{k}$ in the corresponding $q_{k}-p_{k}$ planes, one for each fan. Each subsystem contains all fans except the investigated fan, which is called the reference fan. To derive the relation between the subsystem pressure and the subsystem air quantity, Wang (1992) established the relationship between the subsystems in the multiple-fan system by using these procedures:

- The number of fundamental meshes $M$ is given by $M=n_{b}-n_{n}+1$

- Choose a spanning tree with fan branches being chords designated $1, \ldots, \mathrm{F}$

- If the number of meshes $\mathrm{M}$ is bigger than the number of fans in the system, the other chords are designated $\mathrm{F}+1, \ldots, \mathrm{M}$

The remaining branches are tree branches labeled $\mathrm{M}+1, \ldots, \mathrm{n}_{\mathrm{b}}-1$, and the return branch is numbered $\mathrm{n}_{\mathrm{b}}$. For example, to study the subsystem characteristic for fan $k$, fan $k$ is replaced by a head or quantity source. The subsystem $k$ for fan $k$ contains all fans in the system except the fan No. (k). Then, for each solution curve $L_{k}$ of the subsystem k, the subsystem can be represented by the following equations. 


$$
\begin{array}{ll}
f_{i}\left(q_{1}, \ldots, q_{M}\right)-t_{i}\left(q_{i}\right)=0 & \mathrm{i}=1, \ldots, \mathrm{F} ; \mathrm{i} \neq \mathrm{k} \\
f_{i}\left(q_{1}, \ldots, q_{M}\right)-P_{i}=0, & \mathrm{i}=\mathrm{k} \\
f_{i}\left(q_{1}, \ldots, q_{M}\right)=0, & \mathrm{i}=\mathrm{F}+1, \ldots, \mathrm{M}
\end{array}
$$

where $t_{i}$ is the pressure gain from a fan $i$, and

$$
f_{i}=\sum_{j=1}^{B} b_{i j} r_{j}\left|\sum_{k=1}^{M} b_{k j} q_{k}\right| \sum_{k=1}^{M} b_{k j} q_{k}=0 \quad \mathrm{i}=1, \ldots, \mathrm{M}
$$

In equation $2.9, \mathrm{~b}_{\mathrm{ij}}$ is the element of the fundamental mesh matrix; its value equals $1,-1$ or 0 .

These three types of equations represent $M$ equations in $(M+1)$ unknown, $\mathrm{q}_{1}$ to $\mathrm{q}_{\mathrm{M}}$ and $\mathrm{P}_{\mathrm{i}}$. To solve this type of equation, we must assume a value for one of the variables and then solve for the remaining variables. The solution curves $\mathrm{L}_{\mathrm{k}}$ for the subsystems are represented in $(\mathrm{M}+1)$ dimensional space. This space is difficult to visualize if the number of meshes (M) in the network is larger than two (Figure 1.2). The subsystem characteristic curve can be represented as:

- $\quad P_{k}$ represents the projection of the solution curve $L_{k}$ with the $q_{k}-p_{k}$.

- $U_{k i j}$ represents the projection of the solution curve $L_{k}$ with the $q_{i}-q_{j}$ plane $(i, j=1, \ldots$, $\mathrm{M} ; \mathrm{i} \neq \mathrm{j})$.

$\mathrm{P}_{\mathrm{k}}$ and $\mathrm{U}_{\mathrm{kij}}$ consist of a series of all the available operating points in the subsystem. Each operating point is equivalent to a solution point found in equations 2.6 to 2.8 when branch $k$ is treated as a fixed-quantity branch. Fan $k$ characteristic curve, $\mathrm{t}_{\mathrm{k}}\left(\mathrm{q}_{\mathrm{k}}\right)$, does not 
appear in any of the previous equations and as a result the subsystem characteristic curve for a fan is independent of its characteristic curve.

\subsection{Graphical Solution of Mine Ventilation Networks}

A graphical solution is applicable only to networks that can be reduced to an equivalent system of two series branches by applying the principle of series and parallel combinations. Wang was the first to investigate the problem of multiple-fan systems. In 1985, he described a graphical technique that can not only display the fan characteristic and subsystem parameters of two-fan ventilation systems, but can also display of fan pressure distributions graphically over a wide range of air quantities without specifying the fan characteristics. The concept of the subsystem has been defined as the pressure-vsquantity curve for an equivalent two terminal associated subsystem element connected to the two nodes of the reference fan branch. Wang developed another representation of the subsystem characteristic curves by using q-q coordinates (Wang, 1984b). The relation between the air quantity, delivered from one fan, to the total air quantity, provided the theoretical basis for this graphical solution. The air quantities of subsystems have been used as coordinates to plot the modified fan curves that intersect at the operating point of the system. In 1988, Wang presented a comprehensive discussion on the classical approach to the graphical solution of ventilation networks. He applied the principle of series-parallel reduction to present a graphical approach to solving to mine ventilation networks. This paper presented a coding scheme that includes a set of 11 basic graphical procedures emphasizing the basis of pressure differences, i.e., pressure loss or pressure gain for the characteristic curves. According to this scheme, the network can be reduced 
to an equivalent system of two series branches, which facilitates obtaining the operating point of the system. In 1993, Wang discussed the concept of the subsystem characteristic curve using a graphical technique that applies to two fans in series-parallel networks. Wang considered the branch that contains a fan to be a fan branch. The multiple-fan network is divided to two components; (a) fan branch, or reference fan branch, and (b) the associated subsystem that contains all other branches of the network including the other fan or fans. As a result, there are $F$ fan branches and $F$ subsystems for the network that contains $F$ fans. This paper proved that in these cases the subsystem curve may not be a strictly increasing curve, may not pass through the origin, and the system may have many operating points depending upon the intersections between the subsystem and reference fan characteristic curves. This is because the fan curves become an element of the subsystem.

English and Wang (1997) have represented a fan head as a positive energy and a mine head as a negative energy in the $\mathrm{p}-\mathrm{q}$ coordinate system. Adding the two curves produces a third curve that intersects with the $\mathrm{X}$-axis (q-axis) at the operating point: the point at which the fan energy input equals the air circulation energy loss. This approach can be extended to the graphical solution of any series/parallel multiple-fan system.

\subsection{Numerical Solution of Mine Ventilation Networks}

Traditional Hardy Cross and Newton-Raphson numerical methods are widely used to solve mine ventilation systems. These methods are simply techniques used for solving systems of nonlinear equations. Each is iterative and begins with an initial guess 
for the solution. Straightforward procedures (standard linear algebra techniques) are applied to obtain a new solution. If the new solution differs from the initial solution by less than a specified amount, then the technique terminates. Otherwise, the new solution becomes the input for another iteration trial and the procedure is repeated. Besides these two popular approaches, various other linearization and optimization techniques can be used to solve mine ventilation network problems.

\subsubsection{Hardy Cross Method}

The most popular technique applied to conventional network analysis has been the Hardy Cross iterative method. This method was used by civil engineers to solve water distribution system problems (Cross, H., 1936). Scott and Hinsley (1951) modified and developed the Hardy Cross method for use in mine ventilation systems. However, it did not become practical for mine applications until the availability of high-speed computers. The writers assume that each equation is a function of only one variable, and use two terms of Taylor's series in the derivative of the improved formula. It is equivalent to Newton's method of tangents applied to each equation individually (Wang 1982a).

This technique of fluid network analysis involves the making of an initial estimate of flow distribution, $\mathrm{q}_{\mathrm{a}}$, calculating an approximate correction to be applied to each branch flow, $\Delta \mathrm{q}$, and repeating the correction procedure iteratively until an acceptable degree of accuracy has been achieved (Figure 2.2). 
It assumes that each equation is a function of only one variable, q. Consider a quantity, q, passing through an airway of a resistance (r) and obeying the square law of mine ventilation $\mathrm{p}=\mathrm{r}|\mathrm{q}| \mathrm{q}$, where $\mathrm{p}$ represents the frictional pressure drop along the airway. In order to determine the true value of the quantity $q$ an estimated value $\mathrm{q}_{\mathrm{a}}$ is first assigned.

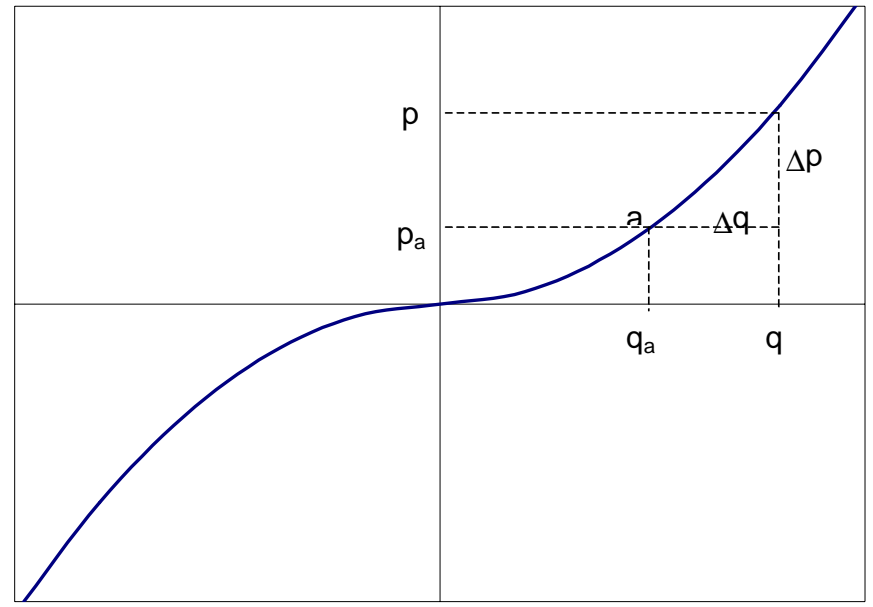

Figure 2.2 Illustrative figure for the Hardy Cross method

$$
q=q_{a}+\Delta q
$$

Where $\Delta \mathrm{q}$ is the correction factor

To get a new value of $\Delta \mathrm{q}$, we may rewrite the square law as

$$
\begin{aligned}
& p=r\left|q_{a}+\Delta q\right|^{*}\left(q_{a}+\Delta q\right) \\
& p=r\left|q_{a}\right|\left(q_{a}\right)+2 r\left(q_{a}\right) \Delta q+r|\Delta q| \Delta q \\
& p_{a}=r\left|q_{a}\right| q_{a} \\
& \Delta p=p-p_{a}=2 r q_{a} \Delta q+r|\Delta q| \Delta q=r|q|(q)-r\left|q_{a}\right| q_{a}
\end{aligned}
$$


The value of $|\Delta q| \Delta q$ is so small compared with the value of $2 \mathrm{q}_{\mathrm{a}} \Delta \mathrm{q}$, that it may be neglected:

$$
\begin{aligned}
& \Delta p=2 r q_{a} \Delta q \\
& \Delta q=\frac{\Delta p}{2 r q_{a}}=\frac{r|q|(q)-r\left|q_{a}\right|\left(q_{a}\right)}{2 r q_{a}}
\end{aligned}
$$

Suppose that air quantities $\mathrm{q}_{\mathrm{j}}\left(\mathrm{j}=1,2, \ldots, \mathrm{n}_{\mathrm{b}}\right)$ are initially assumed such that Kirchhoff's current law is satisfied, assume that the $i^{\text {th }}$ equation in the network is a function of $\mathrm{q}_{\mathrm{i}}$ only, where $\mathrm{q}_{\mathrm{i}}$ is the air quantity for the chord contained in mesh $\mathrm{i}$. By considering the effect of the fans in the fan branches and the effect of natural ventilation, $\mathrm{H}_{\mathrm{nj}}$, the correction value of mesh i can be estimated follows:

$$
f_{i}\left(q_{i}\right)=\sum_{j=1}^{n_{b}} b_{i j} r_{j}\left|q_{j}\right|\left(q_{j}\right)-H_{n j}-t_{j}\left(q_{j}\right)
$$

Where $b_{i j}$ is the element of the fundamental mesh matrix and $t_{j}$ is the pressure delivered from the fan at air quantity $\mathrm{q}_{j}$. Let $\mathrm{q}_{\mathrm{i}}+\Delta \mathrm{q}_{\mathrm{i}}$ be the next improved value for $\mathrm{q}_{i}$ where $\Delta \mathrm{q}_{\mathrm{i}}$ is the correction factor for the $\mathrm{i}^{\text {th }}$ mesh. Expanding equation (2.15) in Taylor's series and neglecting high-order terms we find:

$$
\begin{aligned}
& f_{i}\left(q_{i}+\Delta q_{i}\right)=f_{i}\left(q_{i}\right)+\Delta q_{i} f_{i}^{\prime}\left(q_{i}\right)=0 \\
& \Delta q_{i}=-\frac{f_{i}\left(q_{i}\right)}{f_{i}^{\prime}\left(q_{i}\right)} \\
& \Delta q_{i}=\frac{\sum_{j=1}^{n_{b}} b_{i j} r_{i}\left|q_{i}\right|\left(q_{i}\right)-r_{j}\left|q_{j}\right|\left(q_{j}\right)-H_{n_{j}}-t_{i}\left(q_{i}\right)}{\sum_{j=1}^{n_{b}} b_{i j}^{2}\left[2 r_{i}\left|q_{i}\right|-\left(\beta_{j}+2 \gamma_{j} q_{j}\right)\right]}
\end{aligned}
$$

According to Kirchhoff's voltage law $\sum \mathrm{r}_{\mathrm{i}}\left|\mathrm{q}_{\mathrm{i}}\right| \mathrm{q}_{\mathrm{i}}=0$ 


$$
\Delta q_{i}=\frac{-\sum_{j=1}^{n_{b}} b_{i j} r_{j}\left|q_{j}\right|\left(q_{j}\right)+H_{n_{j}}+t_{i}\left(q_{i}\right)}{\sum_{j=1}^{n_{b}} b_{i j}^{2}\left[2 r_{i}\left|q_{i}\right|-\left(\beta_{j}+2 \gamma_{j} q_{j}\right)\right]}
$$

Wang and Hartman (1967) developed a computer solution for mine ventilation networks using the Hardy Cross method. This program employs natural splitting for networks that have fans, natural ventilation, or both. The authors note that the rate of convergence is dependent not only upon the initial guess of the solution, but also upon the selection of the meshes. To obtain a rapid convergence, they construct the fundamental-mesh matrix such that the branches with high resistance factors correspond to the chords of the tree and therefore high-resistance branches are not repeated in the meshes.

Wang and Saperstein (1970) created a computer program that uses the GaussSeidel iteration technique with the Hardy Cross method as a predictor of the solution. This program can handle free-splitting networks, external or internal fans, natural ventilation, and fixed quantity branches. In 1987, Mcllroy derived unrealistic pressure and quantity values for the fans when trying to apply the algorithm on large complex networks. He overcame this difficulty by plotting the full fan curve using a curve-fitting routine and by then constructing fan points outside the normal operating range to obtain better control of the extrapolation. 
Jacques (1976) developed the theoretical basis to generalize the Hardy Cross method in mine ventilation networks. In his paper, he presented a more general program elaborated from a number of requirements, and he answers three

- The aerodynamic resistance of a branch is unknown, but its airflow quantity or pressure drop between its nodes is known.

- The pressure difference between the nodes of a branch is unknown, but the resistance value $R_{a}$ of the branch is known.

- All physical properties -resistance, pressure drop, and air quantity- are known for one branch while they are unknown for another branch.

Barnes (1976) created a Mine Ventilation Network Analysis (MVNA) computer program that applies the Hardy Cross method to balance the airflow in the network. Although this program had been designed for use by those whom have a limited understanding of ventilation, it also facilitated and simplified the calculation and control of mine ventilation networks for the experienced engineer.

Gregory et al., (1987) developed a solution algorithm for arbitrary flow networks based upon the Hardy Cross technique. They introduced a ventilation network analytical method that used a successive numerical correction at nodes instead of the classical method which uses a successive correction of the flow in the branches to achieve balance.

Mutmansky and Kim (1992) applied three mesh-ordering procedures (random method, bandwidth reduction method and profile reduction method) combined with three 
mesh selection methods (minimum-resistance spanning tree, shortest path and minimumresistance-path) to four mine ventilation network solution methods (Newton-Raphson, Hardy Cross, Linear theory, and second-order approximation). The Hardy Cross method is the most efficient solution method when mesh ordering and mesh selection is applied. According to Mutmansky and Kim, the best combination appeared to be the Hardy Cross method with the minimum-resistance-path method and a random ordering of meshes. The Hardy Cross method shows elements of instability when the minimum-resistance spanning tree method is used, but benefits greatly in stability and total computing time when the minimum-resistance-path method is used for mesh selection.

Wang and Tominaga (1996) developed an iterative method for solving mine ventilation networks that contain the $\Delta$-circuits (two-leg) that can not be reduced by applying series-parallel principle. In each iterative cycle, each $\Delta$-circuit is reduced to a Y-circuit (three-terminal circuit), with the apparent resistances at the current terminal air quantities computed Figure (2.3). If the $\Delta-Y$ and the series parallel operations reduce the total network into a single branch, the improved value for air quantity is obtained by applying the fan pressure and a backward substitution. Otherwise, the resulting nonseries parallel network is solved by the Hardy Cross method utilizing the apparent resistance.

Bernardo and Gama (1997) presented a methodology to design and simulate the underground networks. This method, based on the analysis of real situations, involves two basic steps: first the construction of a complete ventilation database for the mine, 
depending upon a series of surveying studies, second a simulation model using the Hardy Cross method. This simulation model defines the technical-economical feasibility of different solutions of the network.

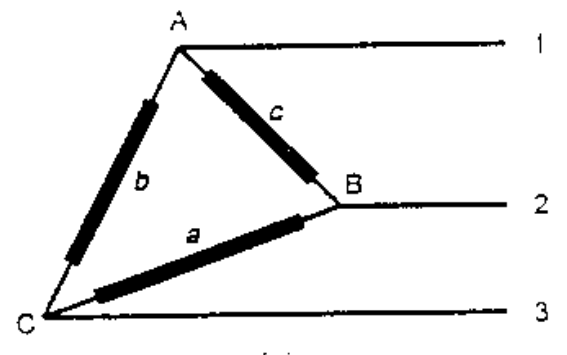

(a)

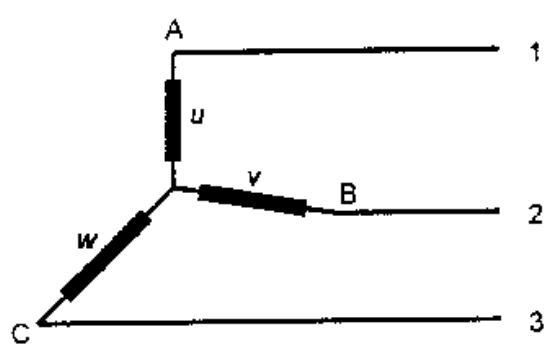

(b)

Figure 2.3 (a) $\Delta$-circuit, and (b) Y-circuit (After Kumazawa, 1942)

Wang (1997) created an algorithm for computing exact solutions for mesh equations, as an alternative to the approximations found in the iteration framework of the Hardy Cross method. This algorithm constructs alternative mesh equations in quadratic form such that the solution and the derivative evaluated at this solution agree with those of the original mesh equations.

\subsubsection{Newton-Raphson Method}

The Newton-Raphson approach used to solve nonlinear equations consists of guessing a solution, approximating the nonlinear equation near that guess (by a linear equation), and then using that linear approximation to obtain new guesses for the solutions. Newton's method corresponds to approximating the function $f$ by its tangent 
line at a point $\mathrm{x}_{\mathrm{o}}$. The point where the tangent line intersects with the $\mathrm{x}$-axis is taken as the new estimate of the solution, Figure 2.4., Newton's method, has a quadratic rate of convergence, and it converges rapidly once the initial guess $\mathrm{x}_{\mathrm{o}}$ is close to the solution $\mathrm{X} *$. Given an estimate of the solution $\mathrm{x}_{\mathrm{k}}$, the function $f$ is approximated by a linear function, consisting of the first two terms of the Taylor series, for the function at the point $\mathrm{x}_{\mathrm{k}}$. The resulting linear system is then solved to obtain a new estimate of the solution $\mathrm{x}_{\mathrm{k}+1}$. To

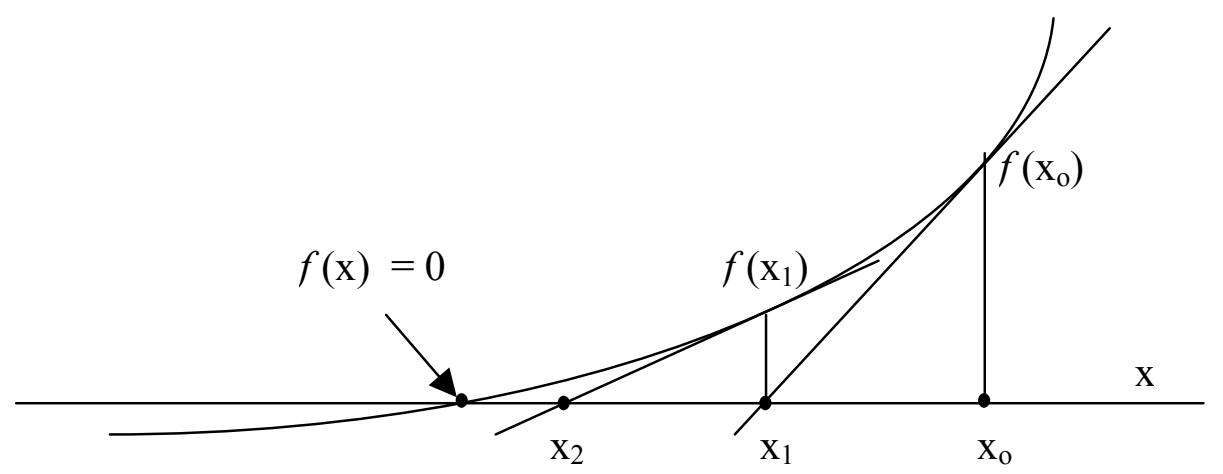

Figure 2.4 Geometric interpretation of Newton's method

derive the formula for Newton's method, first write out the Taylor series for the function $f$ at the point $\mathrm{x}_{\mathrm{x}}$.

$$
f\left(x_{k}+e\right) \approx f\left(x_{k}\right)+e f^{\prime}\left(x_{k}\right) \text {. }
$$

If $f^{\prime}\left(x_{k}\right) \neq 0$ then

$$
e=-f\left(x_{k}\right) / f^{\prime}\left(x_{k}\right)
$$

The Newton equation for one-equation case can then be written by estimating the solution $\mathrm{x}_{\mathrm{k}+1}=\mathrm{x}_{\mathrm{k}}+\mathrm{e}$, where e represents the error:

$$
x_{k+1}=x_{k}-f\left(x_{k}\right) / f^{\prime}\left(x_{k}\right) \text {. }
$$


The one-dimension case represented by Equation 2.20 can be transferred easily to represent $\mathrm{n}$-dimensional case as follows:

Suppose $f(x)=0$ is a system of nonlinear equations which represents

$$
f(x)=f\left(x_{1}, x_{2}, \ldots, x_{n}\right)=\left(\begin{array}{c}
f_{1}\left(x_{1}, x_{2}, \ldots, x_{n}\right) \\
f_{2}\left(x_{1}, x_{2}, \ldots, x_{n}\right) \\
\cdot \\
\cdot \\
\cdot \\
f_{m}\left(x_{1}, x_{2}, \ldots, x_{n}\right)
\end{array}\right)
$$

Where each function $f_{i}$ is a real-valued function, then $\nabla f$ is the matrix with entries $(\nabla f(x))_{i, j} \equiv \frac{\partial f_{j}(x)}{\partial x_{i}}$. The jacobian of $f$ at the point $\mathrm{x}$ is defined as $\nabla f(x)^{T}$, where the $\mathrm{j}$-th column of $\nabla f$ is the gradient of $f_{j}$.

As in one dimension case, the Taylor series approximation for $f$ at the point $\mathrm{x}_{\mathrm{k}}$ is

$$
f\left(x_{k}+e\right) \approx f\left(x_{k}\right)+e \nabla f\left(x_{k}\right),
$$

Where e is now a vector

$$
\begin{aligned}
& f\left(x_{*}\right) \approx f\left(x_{k}\right)+e \nabla f\left(x_{k}\right)^{T}=0, \\
& e=-\nabla f\left(x_{k}\right)^{-T} f\left(x_{k}\right),
\end{aligned}
$$

The new Newton's equation in the n-dimensional case of the solution is then

$$
x_{k+1}=x_{k}+e=x_{k}-\nabla f\left(x_{k}\right)^{-T} f\left(x_{k}\right) \text {. }
$$

Where $\mathrm{x}_{\mathrm{k}+1}, \mathrm{x}_{\mathrm{k}}$ and e are $\mathrm{n}$ dimension vectors (Nash and Sofer, 1996).

Wang (1989) has described a procedure for solving a generalized system of $\mathrm{n}$ network equations in $\mathrm{n}$ unknowns by using the Newton-Raphson method. He classified the network branches into four types: fixed, non-fixed, p-fixed and q-fixed based upon 
whether variable pressure (p) or quantity (q) is dependent or independent. In 1990, Wang presented a generalized mine ventilation network problem formulation and solution algorithm based on the (Newton-Raphson) method. To have a unique solution, the Jacobian matrix of the network equations must be non-singular. Accordingly, Wang summarized the conditions that must be met in the network problem to have a unique solution. These are:

- The number of branches with independent air quantities must be less than, or equal to, the number of chords with respect to a spanning tree.

- The sub-network of branches with independent air quantities must not contain any cutset (will be described in art 3.3).

- The sub-network of branches with dependent control device pressure must not contain any cutset.

\subsubsection{Other Mathematical Methods}

Collins et al., (1978) presented a new approach involving optimization techniques for solving classical pipe network analyses. Pairs of different optimization models were illustrated. The first model, called the content model, determines a set of flows that both satisfy flow conservation and minimize system content. The second model, called co-content, finds a set of head losses that sum to zero around all closed loops. Three mathematical programming algorithms for solving this problem, FrnakWolfe, piece-wise linear approximation, and convex simplex methods have been coded. 
Wang (1982b) applied the Critical Path Method (CPM) to mine ventilation problems that have controlled flow branches. In this paper, Wang introduced the concept of a cutset and defined it as any minimum set of branches in the network that, when cut or removed, divides the network into two distinct sub-networks. By using cutset operations, alternative solutions from the two longest-path tree solution can be obtained. Wang and Mutmansky (1982) once again presented the application of CPM method to mine ventilation problems in a non-mathematical manner. The advantages of using the CPM program are that it:

- Provides a basis for optimizing power consumption in mine ventilation networks,

- Gives a ventilation planner a maximum degree of variation in the selection of main airways,

- Minimizes the number of devices that introduce artificial heads in the network, and

- Gives alternative solutions for the optimum power consumption and relocation or exchange of regulators by fans, or both.

Wang (1984a) formulated the natural air splitting problem in mine ventilation as a nonlinear programming problem by applying the theorem of content for nonlinear systems. As a result, the problem is converted to an unconstrained optimization (minimization) problem of nonlinear programming. Ueng and Wang (1984) adopted the conjugate gradient algorithm in their computer program to solve the ventilation network problems. Since the conjugate method is quadratically converged, it converges very rapidly, normally taking only three iteration steps to achieve acceptable results. 
Bhamidipati and Procarione (1985) presented a geometric mean correction that can reduce the number of iterations by applying linear theory to mine ventilation network problem. They began their analysis by using a standard set of nonlinear equations and then used the concept of conservation of energy to approximately linearize them. This system of equations is then iteratively solved and the coefficients corrected till convergence is obtained. Two methods are used; chord flow rate and nodal head pressure. Although the method of nodal head equations it takes half as much computational time per iteration as the method of flow rate equations, this is less of a concern with today's computational resources.

Tominaga et al., (1985) identified three methods for solving ventilation network problems; the Hardy Cross iterative method, the $\Delta$-Y transformation method and the node potential method. They concluded that the correction values of airflow rate for a basic mesh can be estimated as a root of an equation from a parabola derived from Kirchhoff's second law. Convergence is dependent upon the order chosen for applying the basic meshes.

$\mathrm{Hu}$ and Longson (1990) introduced a new method for calculating the optimal airflow in an established mine ventilation network in which the airflow in certain branches has been specified. They attempted to reduce the size and complexity of the problem, describing the Kirchhoff's Voltage Law (KVL) constraint, by using the bath matrix instead of using the regulator parameters. Following this, they applied the sequential unconstrained minimization technique (SUMT) to solve the minimization 
model with nonlinear inequality constraints. This approach allows for the determination of optimum flow without regard to fans or regulators. The potential benefit of the optimal solution over the free-splitting is dependent upon the requirement of fixed quantity branches in relation to the network geometry.

Kamba et al., (1995) examined the possibility of applying the simplex method, "a linear algorithm approach" and the least-square approach to mine ventilation network problems. They presented a good list of modeling assumptions including incompressibility, fully developed turbulence, leakage, thermal transfer and more.

\subsection{Review of Numerical Solutions for Multiple-Fan System}

Multiple-fan systems have concerned researchers for a long time. Much work has been done to not only formulate and understand the effect of each fan in the system upon the other fans, but also to study the relation of the connection between fans and the shape of the subsystem characteristic curve of each. An extensive literature review has been presented here to uncover the past works that relate to the problem of a multiple-fan systems and their stability.

Mishra et al., (1978) defined a subsystem as the area of influence of each fan within the total mine. The total mine ventilation, however, consists of several interconnected subsystems where the behavior of one subsystem is dictated and influenced by the others. Wang (1983) presented a generalized analysis for the problem of equalizing fan pressure associated with multiple-fan ventilation networks to minimize 
the power consumption in the system. In this paper, Wang defined a major-cutset as the cutset that contains the return dummy branch as its only negative branch. Using this major cutset, alternative solutions can be obtained for optimal solutions by applying a multiple-fan system instead of assuming a single fan (this concept will be illustrated in details in section 3.4. Wang (1984b) analyzed the relationships among the principle parameters for multiple-fan systems. He considered a general multiple-fan system where a fan is located in each of the positive branches in the major cutset. He defined the subsystem with the associated parameters, $\mathrm{Q}_{\mathrm{i}}, \mathrm{H}_{\mathrm{i}}, \mathrm{Z}_{\mathrm{i}}$, and $\mathrm{R}_{\mathrm{i}}$ or the air quantity, pressure loss, power consumed, and system resistance for the fan (i) in the system respectively. In multiple-fan systems, the resistance for each subsystem and for the total system is not constant. Consequently, the pressure loss of the subsystem or the total pressure of the system does not vary as a square of the corresponding air quantities. The pressure loss can be estimated from the square law (Equation 2.3) only when the total resistance of the system becomes a constant, which can occur when the quantity ratios of the subsystem are held constant. Wang et al., (1985) continued the study of multiple-fan systems and presented a comprehensive discussion on the concept of subsystems. They applied major cutset operations to obtain alternative solutions for multiple-fan networks.

Wang and Reddy (1992) demonstrated numerical and graphical methods used in solving multiple-fan systems through three examples. The authors concluded that the graphical method locates all the operating points of simple networks (which are defined as systems that may be reduced by applying the principles of series-parallel combination to an equivalent system of two series branches). To improve the performance of the 
conventional Newton-Raphson method, they implement it by using a switchingparameter algorithm. Although the improved method succeeded in obtaining all the operating points in their first and second examples, it failed to converge at all operating points in the third example when using only one initial guess for the solution.

Wang (1992) extended the concept of mine characteristic curves to the multiplefan system by using the subsystem based approach. Each subsystem has its own solution curve. The solution curve is generated by replacing the reference fan with a hypothetical pressure source or, alternatively, by adding a hypothetical pressure source without removing a fan. However instead of the single mine characteristic curve $\mathrm{P}$ in the $\mathrm{q}-\mathrm{p}$ plane, there are $F$ subsystem characteristic curves $P_{k}$ in the corresponding $q_{k}-p_{k}$ planes. Equations 2.6 to 2.8 describe the subsystem $\mathrm{k}$ of the solution curve $\mathrm{L}_{\mathrm{k}}$. These three types of equations represent $\mathrm{M}$ equations in $\mathrm{M}+1$ unknowns. To solve these types of equations we must assume one of the variables and solve for the others. The solution curves $L_{k}$ for the subsystems are represented in the $(M+1)$ dimensional space.

Lin and Wang (1993a) combined the Quasi-Newton algorithm with the switching parameter method to ensure a continuous search along an augmented-system solution curve in multiple-fan systems. In spite of the effectiveness and the efficiency of this algorithm, it failed to identify all the seven operating points in the second example when using one initial guess for the solution. Lin and Wang (1993b) applied their algorithm to study the basic concepts of subsystems. They have suggested two methods of defining the operating points in any subsystem. In the first method, the traditional approach, the 
operating points are defined by the intersection between the fan curve and its subsystem characteristic curves in the q-p plan. In the second method, the operating points are obtained from the common intersections of the projections of all the subsystems solution curves onto a quantity-quantity plane. They concluded that the subsystem characteristic curve has no fixed shape, it may take a zigzag shape, and it does not pass through the origin.

Huang et al., (1993) and Lin (1995) experimentally investigated the relationship between two subsystems A and B. The authors used two adjustable speed axial flow fans to simulate a two-fan system. The subsystem "A" was constructed by running fan "B" at a constant speed between two terminals resistance. Airflow and the corresponding pressure, at each speed variation of fan "A", were measured. From these measurements, the writers confirmed that the subsystem characteristic curve does not pass through the origin, is not a strictly increasing function (as in a single fan system), and possesses a zigzag portion. These two phenomena are attributed to having fan(s) inside the subsystem. However when fan "B" operated within the strictly decreasing part of its curve, the subsystem characteristic curve passed through the strictly increasing portion. Thus, the subsystem is related to the actual operating range of the fan(s) inside.

Kumar et al., (1995) studied optimum combinations of fan pressures so that the assigned air quantities in the fixed quantity branches minimize the total required air power of the ventilation system. The authors applied the FANVENT simulator to a network consisting of 62 branches, 6 working area, 39 nodes, and two main surface fans. 
The problem was solved three times by using surface fans with regulators, by allowing a booster fan in one branch, and by allowing booster fans in two branches. They concluded that the critical path method can be used to find the optimum combination of surface fan pressures. Also, nonlinear optimization techniques can be used to establish the optimum combination of booster fan pressures. The total air power when applying surface fans was found to be nearly twice as much as when using booster fans.

\subsection{Conclusions Based Upon Literature Review}

A collection of nodes and branches is called a linear graph of real mine ventilation system. The combination between a fan, or a collection of fans, and the mine ventilation system represents a real world mine ventilation system. The equilibrium condition between a fan and a mine is known as the operating point of a mine ventilation system. At this point, the head generated by the fan (pressure gain) is equal to the mine head (pressure loss).

Mine characteristic curves, in single fan systems, are strictly increasing functions where mine resistance is a constant value and can be expressed analytically in the form of Atkinson's equation. The operating point's head and quantity can be obtained directly from the intersection of fan and mine characteristic curves. In a single axial flow fan system, multiple operating points can be avoided by changing fan setting angles, fan diameters and/or fan speeds. Traditional Hardy Cross and Newton-Raphson numerical methods are widely used to solve mine ventilation systems, and other various linearization and optimization techniques exist that can also be used. 
Fans can be connected in series, parallel or combination. The system resistance offered to each of those fans becomes a function not only of the network geometry but also the location and operating characteristics of other fans in the system. Consequently, each fan in the system has its own mine characteristic curve or subsystem characteristic curve. A graphical solution is applicable only to the network that can be reduced to an equivalent system of two series branches by applying the principle of series and parallel combinations. Complex multiple-fan networks can be represented by applying the principles of mine characteristic curves to multiple-fan systems using the subsystem approach. Each subsystem has its own solution curve. The solution curve is generated by replacing the reference fan by a hypothetical pressure source or, alternatively, by adding a hypothetical pressure source without removing a fan. However instead of the single mine characteristic curve $\mathrm{P}$ in the q-p plane, there are $\mathrm{F}$ characteristic curves $\mathrm{P}_{\mathrm{k}}$ in the corresponding $\mathrm{q}_{\mathrm{k}}-\mathrm{p}_{\mathrm{k}}$ planes. Each subsystem contains all fans except the investigated fan and the reference fan. The solution curves $L_{k}$ for the subsystems are represented in the $(\mathrm{M}+1)$ dimensional space. This space is difficult (or even impossible) to visualize, if the number of meshes $\mathrm{M}$ in the network larger than two. 


\section{CHAPTER 3 \\ MODIFIED HARDY CROSS AND NEWTON METHODS}

\subsection{Introduction}

In single or multiple-centrifugal-fan (s) systems, as shown in Figure 1.1, there is only one operating point. However, in a single-axial flow-fan system, unstable conditions can be avoided by restricting fan operation to the right of its stall point, "the normal operating range". Engineers can plot the combined characteristics for axial flowfans in series-parallel network in a relatively straightforward manner, and thus avoid conditions that would cause instability in the system (Wang 1993). In complex multiple axial flow-fan ventilation networks, the same ability to plot the combined fan characteristic curve does not exist.

Wang (1993) has defined the concept of subsystem characteristic curve for a multiple-fan ventilation network in the following way:

"By considering fans as the individual network branches, the multiple-fan network is viewed as consisting of two components: any one fan branch, which is called the reference fan branch, and its associated subsystem. Thus, F subsystem can be designated for the network with $\mathrm{F}$ fan branches. Each subsystem contains one or more fan branches. The subsystem characteristic curve is the pressure-vs.-quantity curve of the subsystem."

The subsystem characteristic curve is not a strictly increasing function nor does it pass through the origin; moreover, it does not have a specific shape. Since an operating 
point is represented by the intersection between the characteristic curve for the subsystem and its reference fan branch, multiple intersections may occur representing multiple operating points. The number of operating points in multiple-fan systems is unknown. The main problems in multiple-fan ventilation systems are tracing a subsystem characteristic curve and defining all the operating points of the system.

Traditional numerical mathematical models such as the Hardy Cross and NewtonRaphson methods cannot trace the subsystem characteristic curves. These conventional numerical methods, even after implementation of the switching parameters technique, cannot be directly applied to multiple-fan network problems. Some modifications must be applied to these methods, as well as to the switching parameter, to effectively trace the solution curves. Hardy Cross and Newton-Raphson methods have been modified to maintain the pre-assigned airflow quantity through the fixed-quantity branch without allowing for any construction of extra regulators or fans in the system. In the same way, switching parameter methods have been modified to trace similar variables in all available directions.

A multiple-fan system is represented by a set of $M$ equations in $M+1$ unknowns. To solve these types of equations, one of these unknowns must be assumed. According to the switching parameter algorithm, there is no specific independent parameter along the solution curve which we can assume, but there is an independent parameter at each iteration step (Chua and Ushida, 1976). In this study, the independent parameter is treated as a fixed-quantity branch. This fixed-quantity branch is given a fixed interval 
$\Delta \mathrm{q}$, which can be a positive (increment) or negative (decrement) according to the direction of the solution curve at each iteration step. One of the implemented numerical algorithms is applied to the system of equations, similar to equations $2.6-2.8$, with a fixed quantity branch until they converge to an adequate solution, which represents a point in the solution curve of the subsystem. These two steps, choosing the fixedquantity branch and applying the implemented numerical method, will be applied to the multiple-fan system equations until all the solution points have been defined.

\subsection{Switching Parameter Algorithm}

Switching parameter is an efficient tool used to find multiple solutions to a system of nonlinear algebraic equations (Chua and Ushida, 1976). This algorithm solves an associated system of first order nonlinear differential equations. The independent variable in these nonlinear equations is not fixed and can be changed from one variable to another. However, to avoid retracing portions of the solution curve a good strategy is to choose the independent parameter at each point on the solution curve to be the variable that changes most rapidly. For example, consider the relation between any two variables $\mathrm{x}$ and $\mathrm{y}$, Figure 3.1. At point (e) and/or point (f) on the curve, the parameter $\mathrm{x}$ is taken as the independent variable since $|\Delta x|>|\Delta y|$ about a small neighborhood of points (e) and (f). On the other hand, y is taken as the independent variable at point (g) and (h), since $|\Delta \mathrm{y}|>|\Delta \mathrm{x}|$ about a small neighborhood of points (g) and (h).

As mentioned before, in the switching parameter algorithm the independent parameter must be chosen at each iteration step. The independent parameter will be the 
parameter that changes most rapidly around its neighboring area. To make an accurate comparison between these variables, the parameters should be same type and share the same units. There are three different types of parameters in the multiple-fan resistance $r_{j}$, airflow rates $q_{j}$ and pressure $P_{k}$ of the subsystem. The resistance parameters are assumed given for all airways in this work. The airflow rates and the pressure are the unknowns to be solved for their values. In each subsystem $\mathrm{k}$, there are $\mathrm{M}$ unknown airflow $\mathrm{q}_{\mathrm{j}}$ values plus one unknown pressure $\mathrm{P}_{k}$ value in $\mathrm{M}$ equations. Thus, there are $\mathrm{M}+1$ in only $\mathrm{M}$ equations.

In the case of a single fan system pressure consumed by the system is usually treated as a dependent parameter and airflows as independent parameters, and the subsystem pressure drop $\mathrm{P}_{\mathrm{k}}$ is treated as a dependent parameter. Consequently, in multiple fan systems, to get the variable that has the biggest change around an interval of the subsystem solution curve a comparison will be made between the airflow parameters $\mathrm{q}_{\mathrm{i}}$. 


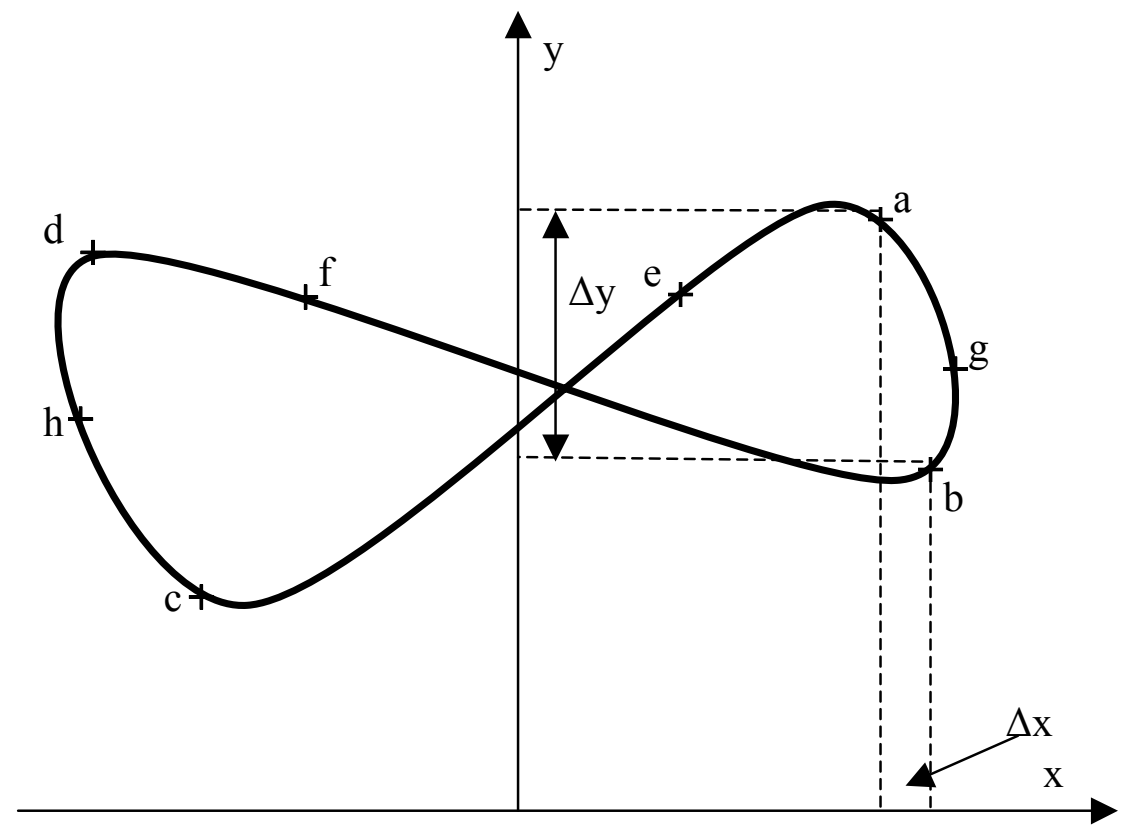

Figure 3.1 Switching Parameter between variables $\mathrm{x}$ and $\mathrm{y}$

\begin{tabular}{|c|c|}
\hline Interval & Search Direction \\
\hline $\mathrm{x}_{\mathrm{c}} \leq \mathrm{x} \leq \mathrm{x}_{\mathrm{a}}$ & $\mathrm{x}$ \\
\hline $\mathrm{y}_{\mathrm{b}} \leq \mathrm{y} \leq \mathrm{y}_{\mathrm{a}}$ & $\mathrm{y}$ \\
\hline $\mathrm{x}_{\mathrm{d}} \leq \mathrm{x} \leq \mathrm{x}_{\mathrm{b}}$ & $\mathrm{x}$ \\
\hline $\mathrm{y}_{\mathrm{c}} \leq \mathrm{y} \leq \mathrm{y}_{\mathrm{d}}$ & $\mathrm{y}$ \\
\hline
\end{tabular}




\subsection{Cutset and Cutset Operations}

Wang (1983) defined the major cutset as "a cutset which contains the return dummy branch as its only negative branch". As shown in Figure 3.2, each set of branches intersected by a broken line is a major cutest. In the major cutset, the sum of the air quantities in the positive branches equals the quantity through the return branch No. 9. Wang has presented the theoretical basis for cutset operations, and discusses the conditions under which the total air power remains unchanged. These conditions can be used to obtain alternative solutions for a multiple-fan system. As an example, a fan installed in the return branch can be replaced by two fans in every branch of cutest [1], branches No. 1 and 2, and by adjusting the pressure to one-half that of the single-fan solution. Seven alternative solutions can be calculated by using another group from the seven available cutsets.

The multiple-fan systems studied in this work are confined to the cases where a set of fans is associated with the major cutset branches. This study considers three conditions in the network. The first condition is that each fan is not only installed in a positive branch in the cutset, but that it is also contained in its mesh. Secondly, each of these meshes contains the return branch and only one positive branch of the cutset. Finally, each positive branch is contained in only one mesh. To ensure this, the spanning tree of the network has been chosen such that the return branch is contained in the tree and branches 1 through $\mathrm{F}$ are contained in the chord set. If the number of meshes is larger than the number of fans (F), the chords that are not in the cutset will be labeled as 
$\mathrm{F}+1, \ldots, \mathrm{M}$. Finally, each fundamental mesh is assigned the same number as the branch number of the chord contained on it.

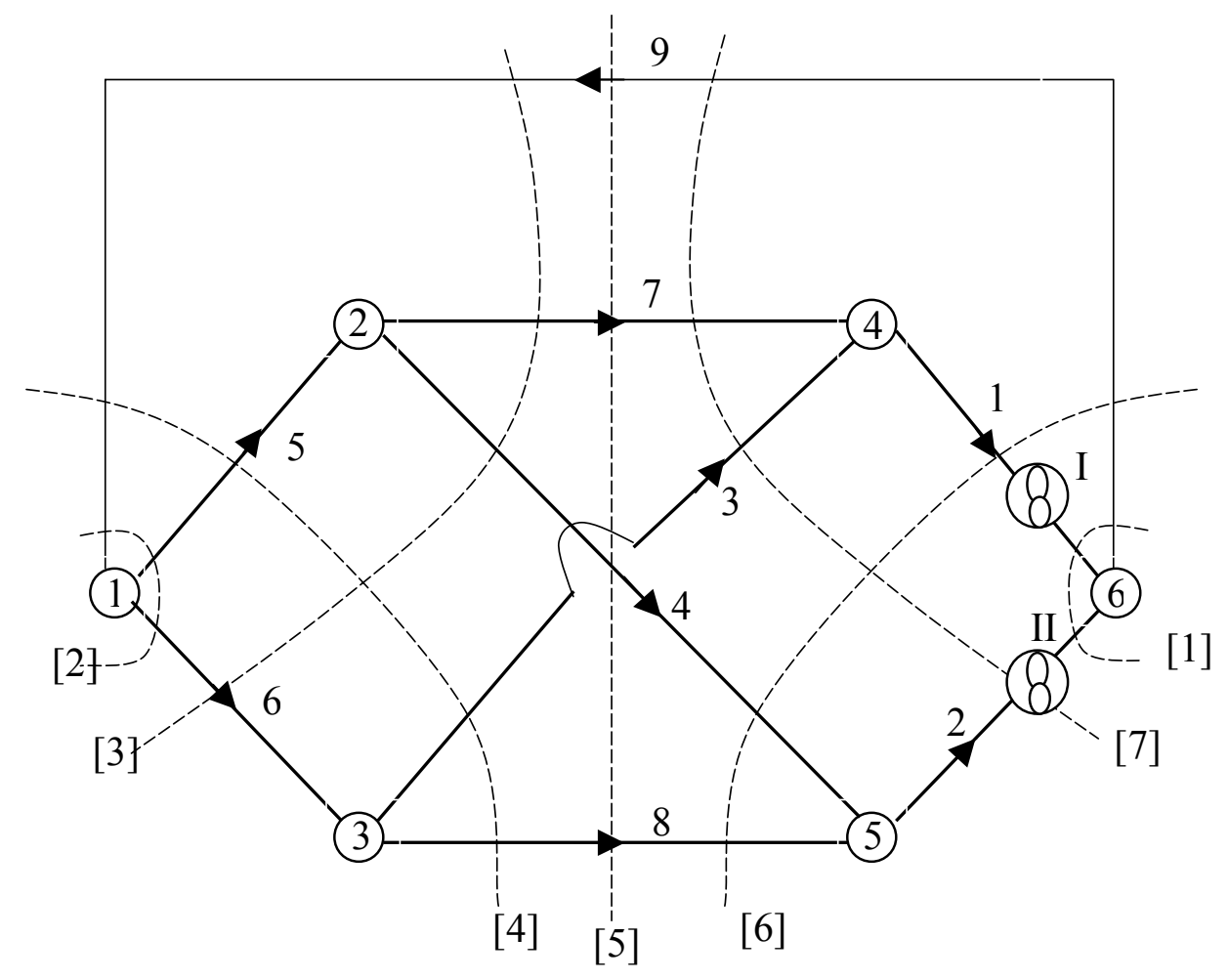

Figure 3.2 Major cutest (Wang, 1983) 


\subsection{Modified Hardy Cross Method}

The Hardy Cross method, as illustrated in part 2.3.1, is an iterative method that is similar to the Guess-Seidel method for solving linear equations. When this ordinary method is applied to fixed-quantity branches, the unknown to be solved is either the regulator head $h_{R_{j}}$ or the fan head $h_{f_{j}}$. However, when it is applied to multiple-fan systems, neither extra fans nor regulators are permitted to be used in any step of the iteration. Moreover, if any kind of pressure regulator (fan or regulator) is used in the iteration steps, it means a different network with different values of resistances will be solved at each iteration step. Thus, instead of using regulators, the pressures gain from the main fans in the main cutset is readjusted to deliver the assigned quantity in the fixed quantity branch. Accordingly, there are two types of fixed-quantity branches in the multiple fan system. The first type is the fixed-quantity branch that lies in the major cutset, which represents the fan branches. The second type is the fixed-quantity branch which lies outside the major cutset. To treat fixed-quantity branches in the major cutset, the fan pressure $\mathrm{t}_{\mathrm{i}}\left(\mathrm{q}_{\mathrm{i}}\right)$ is adjusted to deliver the pre-assigned quantity $\mathrm{q}_{\mathrm{i}}$. Equilibrium between the pressure delivered from the fan (i), calculated from its characteristic equation, and the pressure consumed from subsystem (i), must be iteratively reached. Pressure delivered from a fan depends upon the flow across the fan branch, so it is constant at a fixed fan branch. Pressure consumed across the subsystem $P_{i}$ depends upon the resistances of the branches in this subsystem and the flow across these branches, so it is not constant at a fixed quantity branch and is directly proportional to the square of the flow in each branch. The difference between the pressure delivered from a fan and the consumed pressure across its subsystem is distributed among the fans in the system by 
applying cutset operations. If the fixed quantity branch lies outside the cutset, the value of the regulator pressure will be transferred to the fans in the system by indirect method, as follows:

Assume that $h_{R_{j}}$ is the value of the regulator pressure in branch $\mathrm{i}$.

$$
h_{R_{i}}=\sum_{j=1}^{n_{b}} H_{j}=\sum_{j=1}^{n_{b}} b_{k j} r_{j}\left|q_{j}\right| q_{j}
$$

Where $\mathrm{k}$ is the mesh containing the fixed-quantity branch

$$
h_{R i}^{\prime}=\frac{d h_{R_{i}}}{d q_{i}}=\frac{d\left(\sum_{j=1}^{n_{b}} H_{j}\right)}{d q_{i}}
$$

According to Hardy Cross method, the correction value at each step is equal to $\Delta(q)_{i}$ :

$$
\Delta(q)_{i}=\frac{-h_{R_{i}}}{a b s\left(h_{R i}^{\prime}\right)}
$$

The value of the sum of the pressure drops $\sum_{j=1}^{n_{b}} H_{j}$ around the mesh is distributed among the branches in this loop according to the correction factor. This correction is not be applied to the fixed-quantity branch. Additionally, this correction is applied iteratively until the value of the pressure drops across this closed loop converges to a pre-specified value. Figure 3.3 shows the flow chart of the Hardy Cross with the switching parameter algorithm. This algorithm has been used to solve systems of equations similar to equation 2.6, 2.7 and 2.8. 


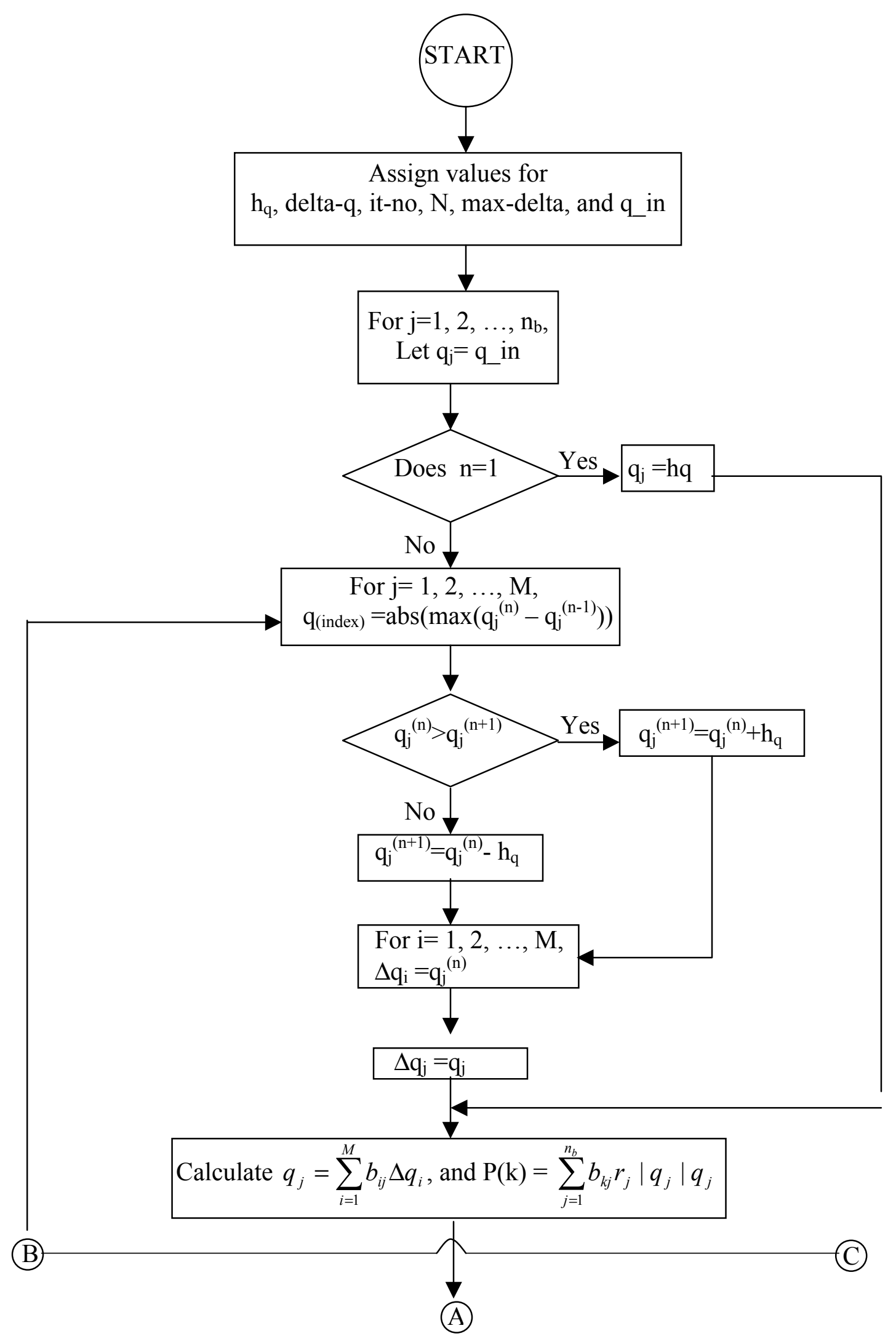

Figure 3.3 -Flow chart of Hardy Cross with the Switching-parameter algorithm 


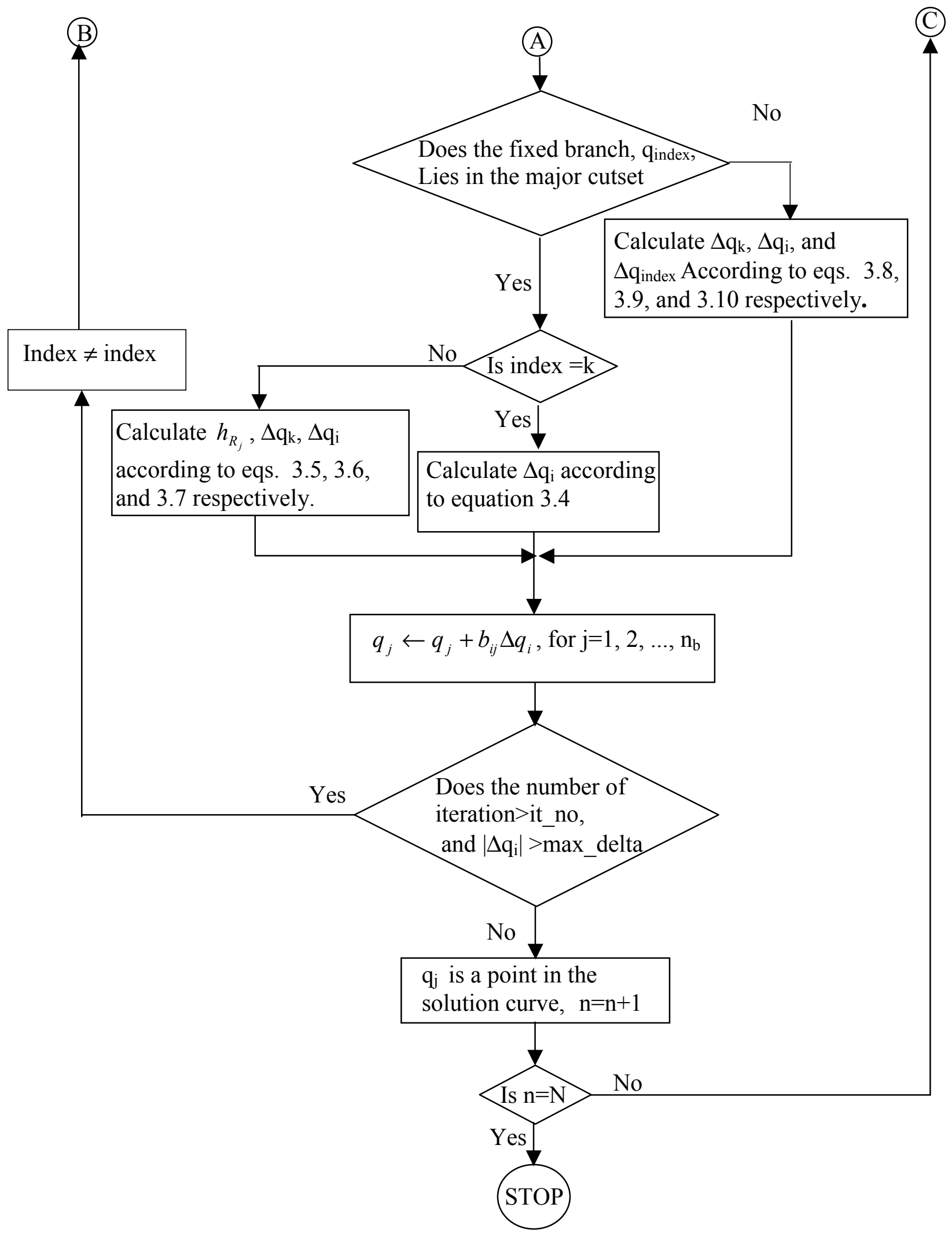

Figure 3.3 continued 
The Hardy Cross algorithm approach can be summarized as follows:

For each subsystem $\mathrm{k}(\mathrm{j}=1,2, \ldots, \mathrm{m})$

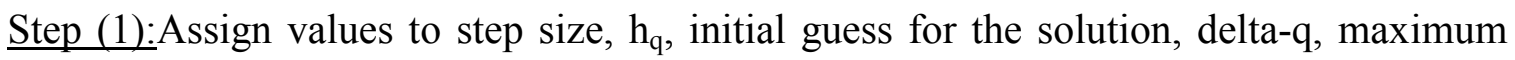
number of iterations, it-no, number of steps, $\mathrm{N}$, tolerance value, max-delta, and the flow through the branches, q in.

$\underline{\operatorname{Step}(2):}$ Let $\mathrm{q}_{\mathrm{j}}=\mathrm{q} \_$in, for $\mathrm{j}=1,2, \ldots, \mathrm{n}_{\mathrm{b}}$.

$\underline{\text { Step (3): }}$

- For the first point in the solution curve, let branch (j), where $\mathrm{j}$ is the subsystem under investigation, as the fixed-quantity branch and let $\mathrm{q}_{\mathrm{j}}=\mathrm{h}_{\mathrm{q}}$.

- For the rest of the points of the solution curve compare between $\mathrm{q}_{j}^{(\mathrm{n})}$ and $\mathrm{q}_{\mathrm{j}}^{(\mathrm{n}-1)}$ and

find branch (index) that has the maximum absolute change $\left(\max \left\{\left|\left[\mathrm{q}_{j}{ }^{(\mathrm{n})}-\mathrm{q}_{\mathrm{j}}^{(\mathrm{n}-1)}\right]\right|\right\}\right)$

$\underline{\text { Step (4): }}$

$$
\text { If } \mathrm{q}_{j}^{(\mathrm{n})}>=\mathrm{q}_{j}^{(\mathrm{n}-1)} \text {, let } \mathrm{q}_{j}^{(\mathrm{n}+1)}=\mathrm{q}_{\mathrm{j}}^{(\mathrm{n})}+\mathrm{h}_{\mathrm{q}}
$$

Else, let $\mathrm{q}_{j}^{(\mathrm{n}+1)}=\mathrm{q}_{\mathrm{j}}^{(\mathrm{n})}-\mathrm{h}_{\mathrm{q}}$

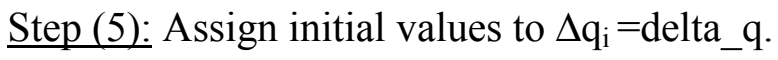

$\underline{\text { Step (6): }}$ Let $\Delta \mathrm{q}_{\mathrm{j}}=\mathrm{q}_{\mathrm{j}}$ at step number $(\mathrm{n}+1)$, for $\mathrm{i}=\mathrm{i}, \ldots, \mathrm{M}$.

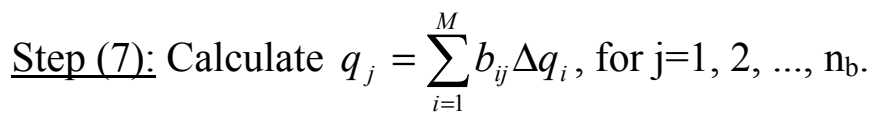

$\underline{\text { Step (8) }}$ for the fixed quantity branch (k), calculate $\mathrm{P}(\mathrm{k})=\sum_{j=1}^{n_{b}} b_{k j} r_{j}\left|q_{j}\right| q_{j}$

Step (9): Perform the following for $\mathrm{j}=1,2, \ldots, \mathrm{M}$;

(I) If the fixed branch (index) is the same as branch (k), adjust fan (k) to deliver the pressure $\mathrm{P}(\mathrm{k})$ and calculate; 
A] $\Delta q_{i}=\frac{-\sum_{j=1}^{n_{b}} b_{i j}\left(r_{j}\left|q_{j}\right| q_{j}-t_{i}\left(q_{i}\right)\right)}{\sum_{j=1}^{n_{b}} b_{i j}\left(2 r_{j}\left|q_{j}\right|-\left[\alpha_{j}+\beta_{j} q_{j}+\gamma_{j} q_{j}^{2}+\delta_{j} q_{j}^{3}\right]\right)}$ where $\mathrm{i} \neq \mathrm{k}$

B] $q_{j} \leftarrow q_{j}+b_{i j} \Delta q_{i}$, for $\mathrm{j}=1,2, \ldots, \mathrm{n}_{\mathrm{b}}$

(II) If the fixed branch (index) lies in the same major cutset with branch (k) calculate;

A] $h_{R_{i}}=\sum_{j=1}^{n_{b}} b_{(\text {index })(j)} r_{j}\left|q_{j}\right| q_{j}-t_{\text {index }}\left(q_{\text {index }}\right)$

B] $\Delta q_{k}=\frac{-\sum_{j=1}^{n_{b}} b_{k j}\left(r_{j}\left|q_{j}\right| q_{j}+h_{R_{i}}-p(k)\right)}{\sum_{j=1}^{n_{b}} b_{k j}\left(2 r_{j}\left|q_{j}\right|\right)}$

C] $\Delta q_{i}=\frac{-\sum_{j=1}^{n_{b}} b_{i j}\left(r_{j}\left|q_{j}\right| q_{j}+X-t_{i}\left(q_{i}\right)\right)}{\sum_{j=1}^{n_{b}} b_{i j}\left(2 r_{j}\left|q_{j}\right|-\left[\alpha_{j}+\beta_{j} q_{j}+\gamma_{j} q_{j}^{2}+\delta_{j} q_{j}^{3}\right]\right)}$

where $\mathrm{i} \neq \mathrm{k} \neq$ index

D] $\quad q_{j} \leftarrow q_{j}+b_{i j} \Delta q_{i}$, for $\mathrm{j}=1,2, \ldots, \mathrm{n}_{\mathrm{b}}$.

(III) If the fixed branch (index) does not lie in the same major cutset with the subsystem fan branch (k) calculate;

A] $\Delta \mathrm{q}_{\mathrm{k}}=\frac{-\sum_{\mathrm{j}=1}^{\mathrm{n}_{\mathrm{b}}} \mathrm{b}_{\mathrm{kj}}\left(\mathrm{r}_{\mathrm{j}}\left|\mathrm{q}_{\mathrm{j}}\right| \mathrm{q}_{\mathrm{j}}-\mathrm{P}(\mathrm{k})\right)}{\sum_{\mathrm{j}=1}^{\mathrm{n}_{\mathrm{b}}} \mathrm{b}_{\mathrm{kj}}\left(2 \mathrm{r}_{\mathrm{j}} \mid \mathrm{q}_{\mathrm{j}}\right)}$ 
B]

$$
\Delta q_{i}=\frac{-\sum_{j=1}^{n_{b}} b_{i j}\left(r_{j}\left|q_{j}\right| q_{j}-t_{i}\left(q_{i}\right)\right)}{\sum_{j=1}^{n_{b}} b_{i j}\left(2 r_{j}\left|q_{j}\right|-\left[\alpha_{j}+\beta_{j} q_{j}+\gamma_{j} q_{j}^{2}+\delta_{j} q_{j}^{3}\right]\right)}
$$

where $\mathrm{i} \neq \mathrm{k} \neq \mathrm{index}$

C]

$$
\Delta q_{\text {index }}=\frac{-\sum_{j=1}^{n_{b}} b_{(\text {index })(j)}\left(r_{j}\left|q_{j}\right| q_{j}\right)}{\sum_{j=1}^{n_{b}} b_{i j}\left(2 r_{j}\left|q_{j}\right|\right)} \text { where index } \neq \mathrm{i} \neq \mathrm{k}
$$

D] $\quad q_{j} \leftarrow q_{j}+b_{i j} \Delta q_{i}$, for $\mathrm{j}=1,2, \ldots, \mathrm{n}_{\mathrm{b}}$.

Step (10): If the number of iterations exceeds the pre-assigned maximum number of iteration, it-no, look for another independent variable (index) and go back to step (5).

Step (11): If $\left|\Delta \mathrm{q}_{\mathrm{i}}\right|$ is less than or equal to the maximum tolerance value, max-delta, for $\mathrm{j}$ $=1,2, \ldots, M$, in step (9), the solution is completed. Save $\mathrm{q}_{\mathrm{j}}$ in an array, $\mathrm{Q}_{\mathrm{j}}(\mathrm{step})$.

Step (12): step $=$ step +1 , go to step (3).

Step (13): If step = maximum number of steps, N, stop.

A computer code for the Hardy Cross algorithm is shown in Appendix B.2. A Matlab language has been used throughout this study.

\subsection{Modified Damped Newton Raphson Method}

Conventional numerical methods that solve single fan systems cannot directly applied to multiple fan system. These methods must be implemented first to solve a group of nonlinear equations that has more than a solution. As an example NewtonRaphson method has been used in this research not only to verify the Hardy Cross method but also to prove the validity of using most of the numerical methods that solve 
single fan system to solve multiple fan system after implementation. Switching parameter algorithm has been used to implement both Hardy Cross and Newton-Raphson methods. The same branch classification system that is used in the Hardy Cross method is used in this algorithm as well. The ordinary Newton-Raphson method, Equation 2.22, is not efficient enough to trace the subsystem characteristic curve because the error in step $n+1$ is larger than the error at step n, which leads to an increase in the residual error of the solution (Conte and de Boor, 1980). A damped Newton-Raphson method shows highly efficient convergence when a proper damped value, $1 / 2^{i}$, has been selected and applied to the new increment (delta) of the solution (Equation 3.11). A value of 0.2 for damping the Newton method was found adequate for this application.

$$
q^{n}=q^{n-1}+\operatorname{delta} / 2^{i}, \text { where } \mathrm{i}=1,2, \ldots
$$

As has been illustrated in part 2.3.2, the Newton-Raphson method is an iterative method that consists of three major steps: guessing a solution, approximating the nonlinear equation near that guess (with a linear equation), and using that linear approximation to obtain a new guess for the solution. These steps are calculated repeatedly until it reaches a tolerance value, which represent a point in the solution curve. As an illustrative example, the computer code for the illustrative example in Figure 3.4, is shown in Appendix B.3.

The three steps in the Newton-Raphson can be summarized as follows:

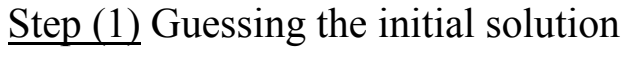


- At the first point of the solution, assign the initial guess of the solution for the independent branches (chords), q, step size, hq, maximum number of iteration, itno, number of steps, s, tolerance value, max-delta.

- For the rest of the solution curve at point $\mathrm{n}$ assign the initial guess of the solution in the chords equal to the value of the solution at point $n-1$, taking into consideration the value of the air quantity at the fixed quantity branch equal to this value at point $\mathrm{n}$ plus the step size hq.

Step (2) Approximating nonlinear equations (Equations similar to 2.6 to 2.8) near the initial guess to linear equations

- Calculate the values of the functions $f(x)$.

- Evaluate the Jacobian matrix $(\nabla f(x))_{i, j} \equiv \frac{\partial f_{j}(x)}{\partial x_{i}}$ of the system of equation $f(x)$.

- Use Guess elimination method to calculate the value of the correction, delta.

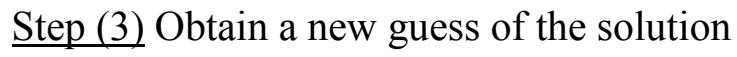

- The damped Newton method is used to calculate the new estimate of the solution according to Equation 3.11.

- New estimate of the solution, $\mathrm{q}^{\mathrm{n}}$, is assigned as a new guess.

- Go to point 2 in step (1) and repeat the algorithm until all the operating points are calculated.

\subsection{Application of the Algorithms to an Administrative Example of Multiple Fan Systems}

To verify the effectiveness of the mathematical models, the network shown in Figure 3.4 has been studied. This network consists of 10 branches, 6 nodes, and 4 fans. 
Two different axial flow fans, fan A and B, have been used as shown in Figure 3.5. A fan type A has been installed in each of branches No. 1 and 3 and a fan type B in each of branches No. 2 and 4. As explained in Chapter 2, the number of chords or number of independent air flow branches, shown as double lines in Figure 3.4, is equal to the number of fundamental meshes, $M=n_{b}-n_{n}+1=5$ branches. Branches 1 to 4 are assigned as fan branches. Branches 1 and 3 have axial flow fans type A and branches 2 and 4 have fans type B. A hypothetical branch, No. 10, is assigned as a return dummy branch. The rest of the branches represent the tree branches. Both the modified Hardy Cross and the modified damped Newton method have been applied to solve this example. The results obtained from the two methods match to a high degree.

Figures 3.6 through 3.9 show both the subsystem characteristic curves in q-p and the subsystem resistance in $\mathrm{r}-\mathrm{q}$ plane for fans No. 1, 2, 3, and 4 respectively. Fans 1, 2 and 4 operate on the right of the stall point within their stable zones, but fan 3 works to the left of its stall point within its unstable zone (as indicated in Figure 3.8 by a dotted circle). None of the subsystem curves pass through the origin and do not have a specific shape. The intersections between any of these subsystems with its fan characteristic curve represent the operating points of the system. At any of these intersections the values of the flow in the chords 1, 2, 3, 4 and 5 can be obtained. Values of the operating points from any of these subsystems must be matched. In this example, one operating point has been recognized. Table (3.1) shows the values of the air quantities at the chords obtained from applying these mathematical models to subsystems 1,2, 3 and 4. 
The projection of the solution plane on the q-q plane can be used to represent the subsystem curve and consequently the operating points of the system (Wang, 1992). Figures 3.10 through 3.15 represent the subsystem characteristic curves in the q-q planes, where $\mathrm{U}_{\mathrm{xyz}}$ indicates the relation between the flow in branch $\mathrm{y}$ and branch $\mathrm{z}$ obtained from subsystem $x$, or its the projection of the solution plane $x$ with the $q_{y}-q_{z}$ plane. All of the subsystem characteristic curves intersect at only one operating point.

Table 3.1-Operating points obtained from the different subsystems.

\begin{tabular}{|c|c|c|c|c|c|}
\hline Subsystem No. & Q1 & Q2 & Q3 & Q4 & Q5 \\
\hline 1 & 32.8 & 26.4 & 15.9 & 28.8 & 20.1 \\
\hline 2 & 32.8 & 26.3 & 15.9 & 28.8 & 20.1 \\
\hline 3 & 32.8 & 26.3 & 15.9 & 28.8 & 20.1 \\
\hline 4 & 32.8 & 26.3 & 15.9 & 28.9 & 20.1 \\
\hline
\end{tabular}




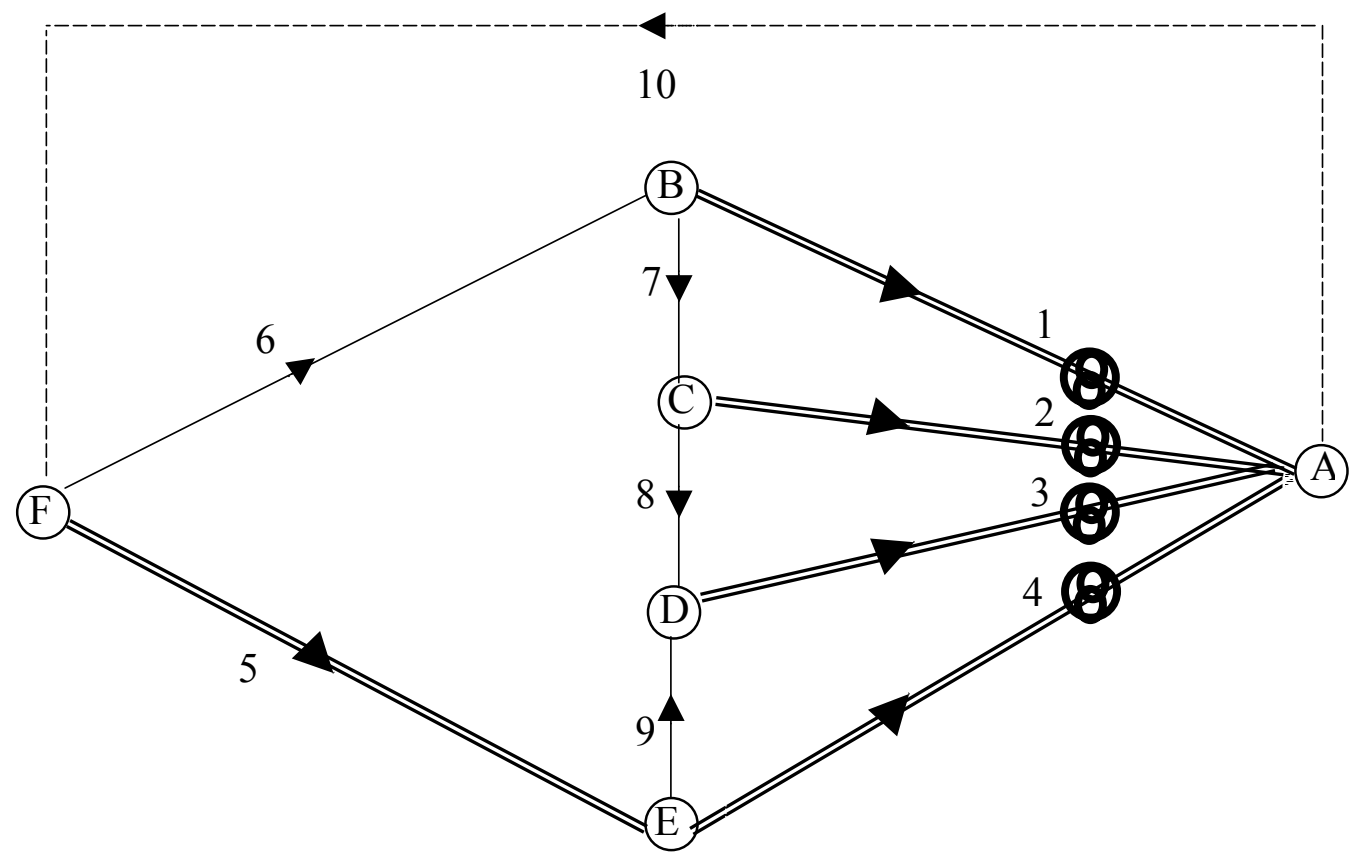

Figure 3.4_The layout and the resistance factors for the preliminary example network.

\begin{tabular}{|c|c|}
\hline Branch No. & Resistance, N.s ${ }^{2} / \mathrm{m}^{8}$ \\
\hline 1 & 0.7 \\
\hline 2 & 0.7 \\
\hline 3 & 0.7 \\
\hline 4 & 0.7 \\
\hline 5 & 0.5 \\
\hline 6 & 0.4 \\
\hline 7 & 0.5 \\
\hline 8 & 0.3 \\
\hline 9 & 0.3 \\
\hline 10 & 0.0 \\
\hline
\end{tabular}




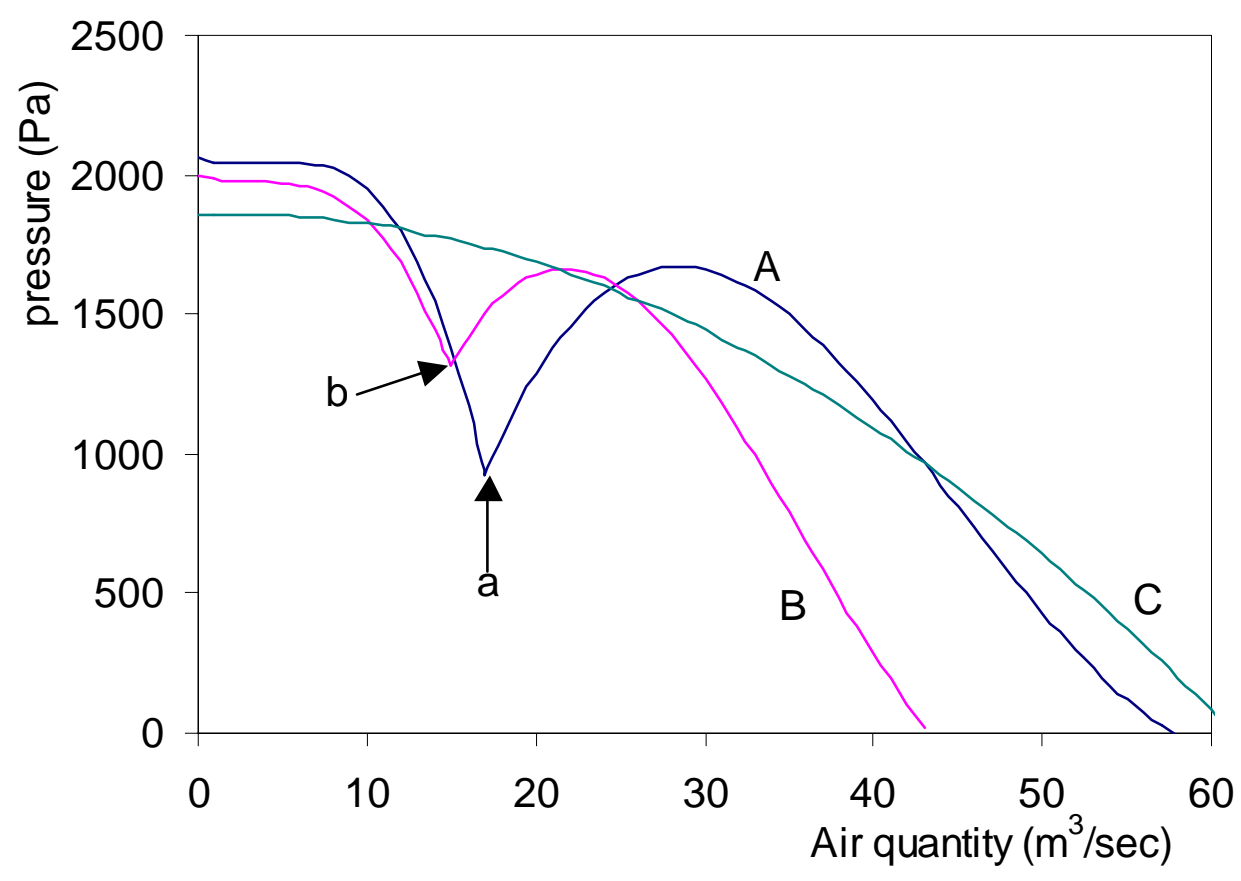

Figure 3.5 -Fan characteristic curves A, B and C

\subsubsection{Approximation of Fan Characteristic Curves}

The two axial flows and one centrifugal fan characteristic curve, designated as A, $\mathrm{B}$ and $\mathrm{C}$ in Figure 3.5, have been used throughout this study. To represent the axial flow fan characteristic curves numerically, for the purpose of computation and also to ensure the differentiability of the fan curve, each fan curve was divided into two segments. Points $\mathrm{a}$ and $\mathrm{b}$ separate the two segments for curve A and B respectively. Then each segment is further divided into a reasonable number of segments (30-40 points). Thereafter each subdivision is approximated with a natural cubic spline (Burden and 
Faires, 1985). During a search routine fan pressure at an air flow, $\mathrm{q}$, which falls into two adjacent points $\mathrm{q}_{\mathrm{i}}$ and $\mathrm{q}_{\mathrm{i}+1}$, is identified by calculating as follows:

$$
T(q)=a_{i}+b_{i}\left(q-q_{i}\right)+c_{i}\left(q-q_{i}\right)^{2}+d_{i}\left(q-q_{i}\right)^{3}
$$

Where $a_{i}, b_{i}, c_{i}$ and $d_{i}$ are the coefficients of the cubic polynomial over the interval of ( $\left.\mathrm{q}_{\mathrm{i}}, \mathrm{q}_{\mathrm{i}+1}\right)$ and can be determined from the natural cubic spline (Wang and Reddy, 1992). 


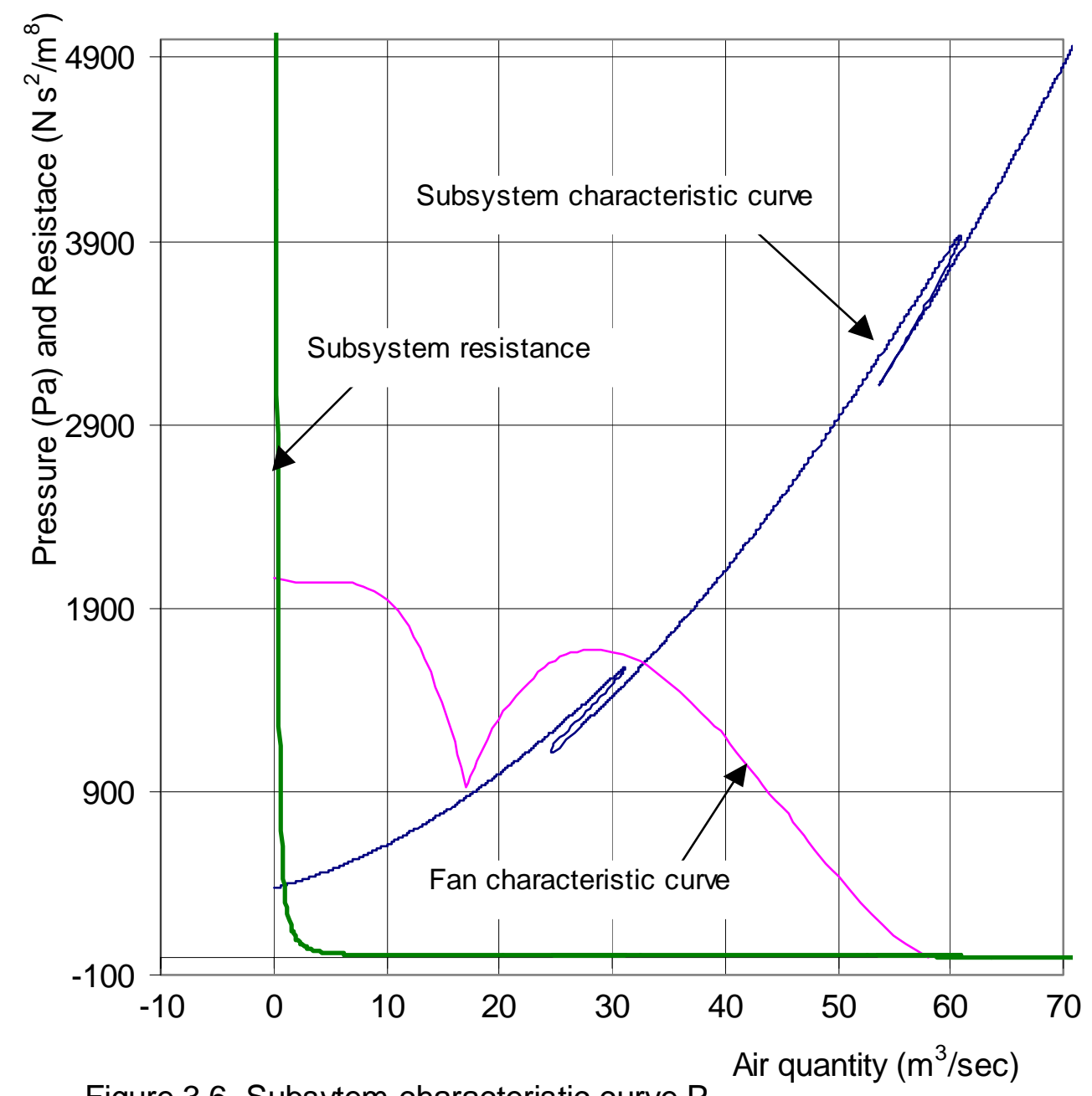

Figure 3.6 -Subsytem characteristic curve $P_{1}$ 


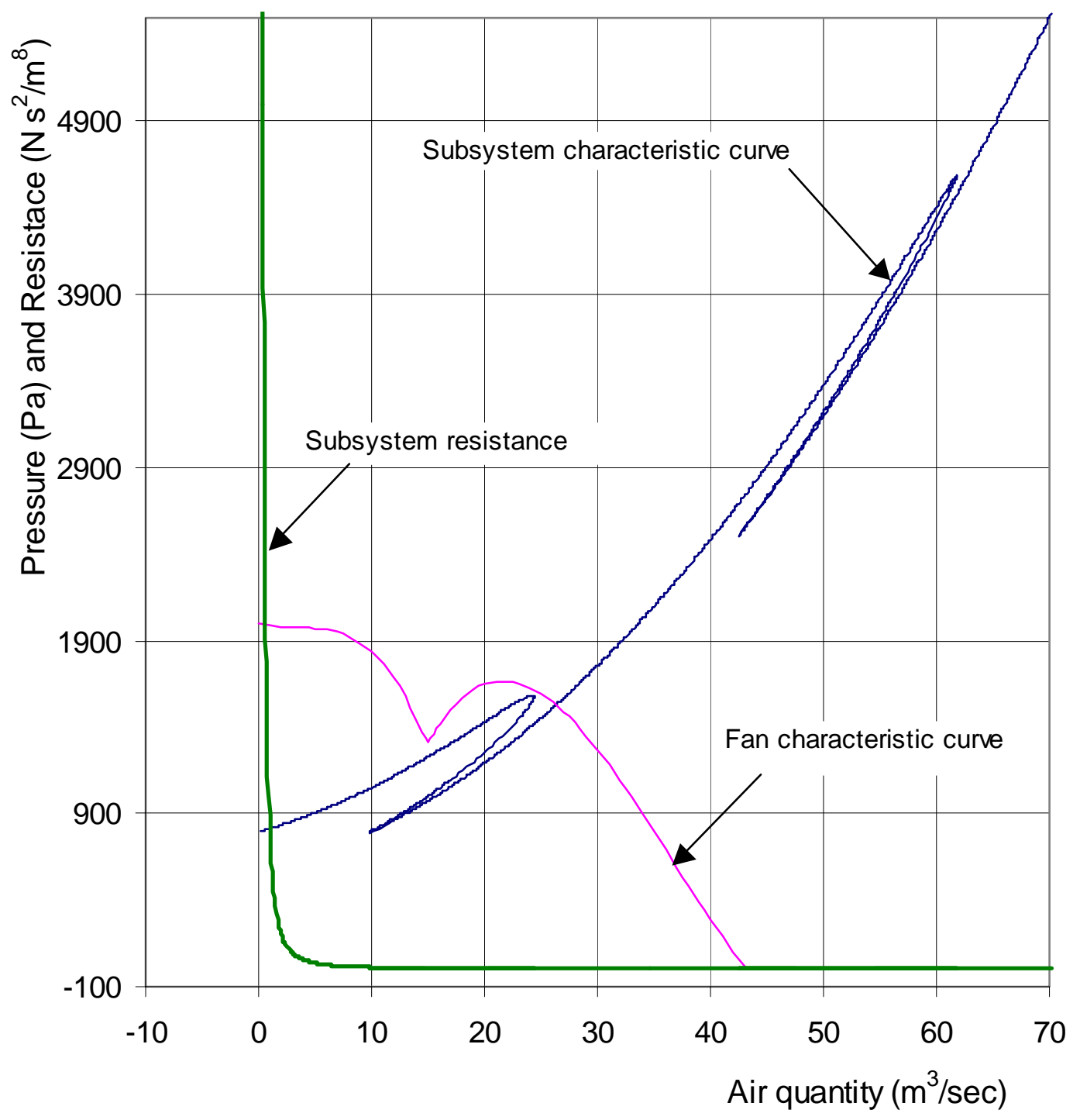

Figure 3.7 -Subsystem characteristic curve $P_{2}$ 


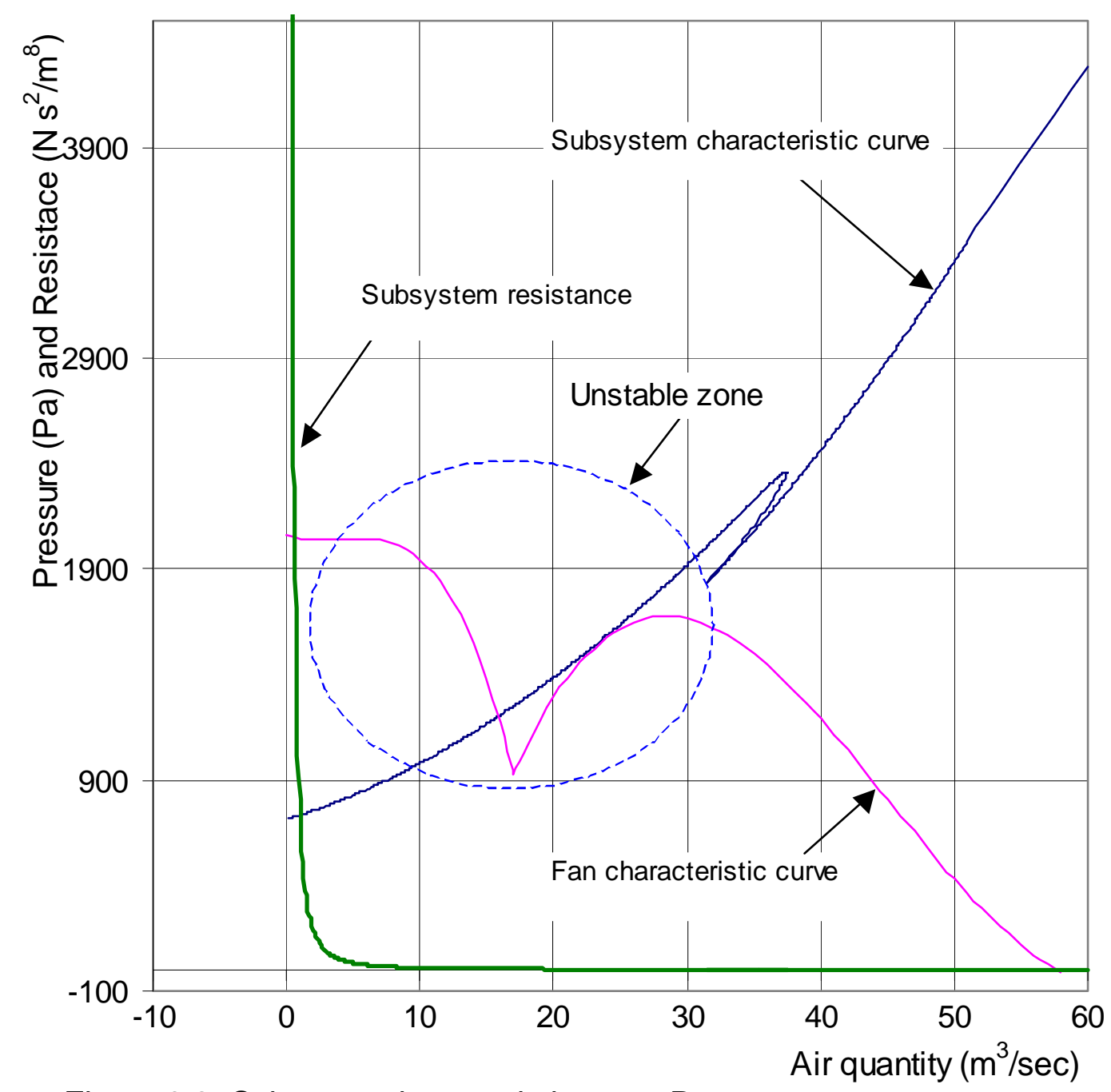

Figure 3.8 -Subsytem characteristic curve $P_{3}$ 


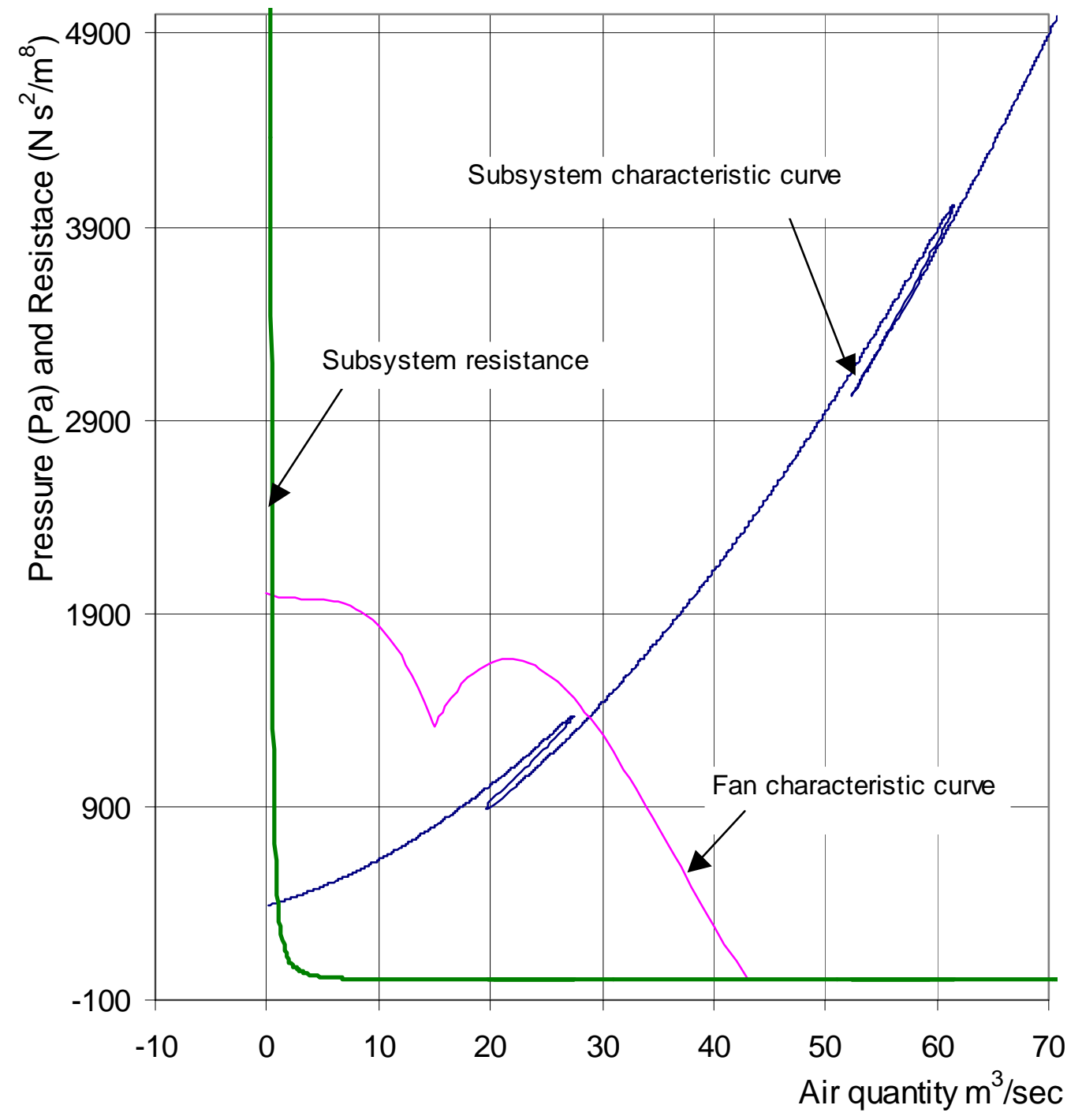

Figure 3.9 -Subsytem characteristic curve $\mathrm{P}_{4}$ 


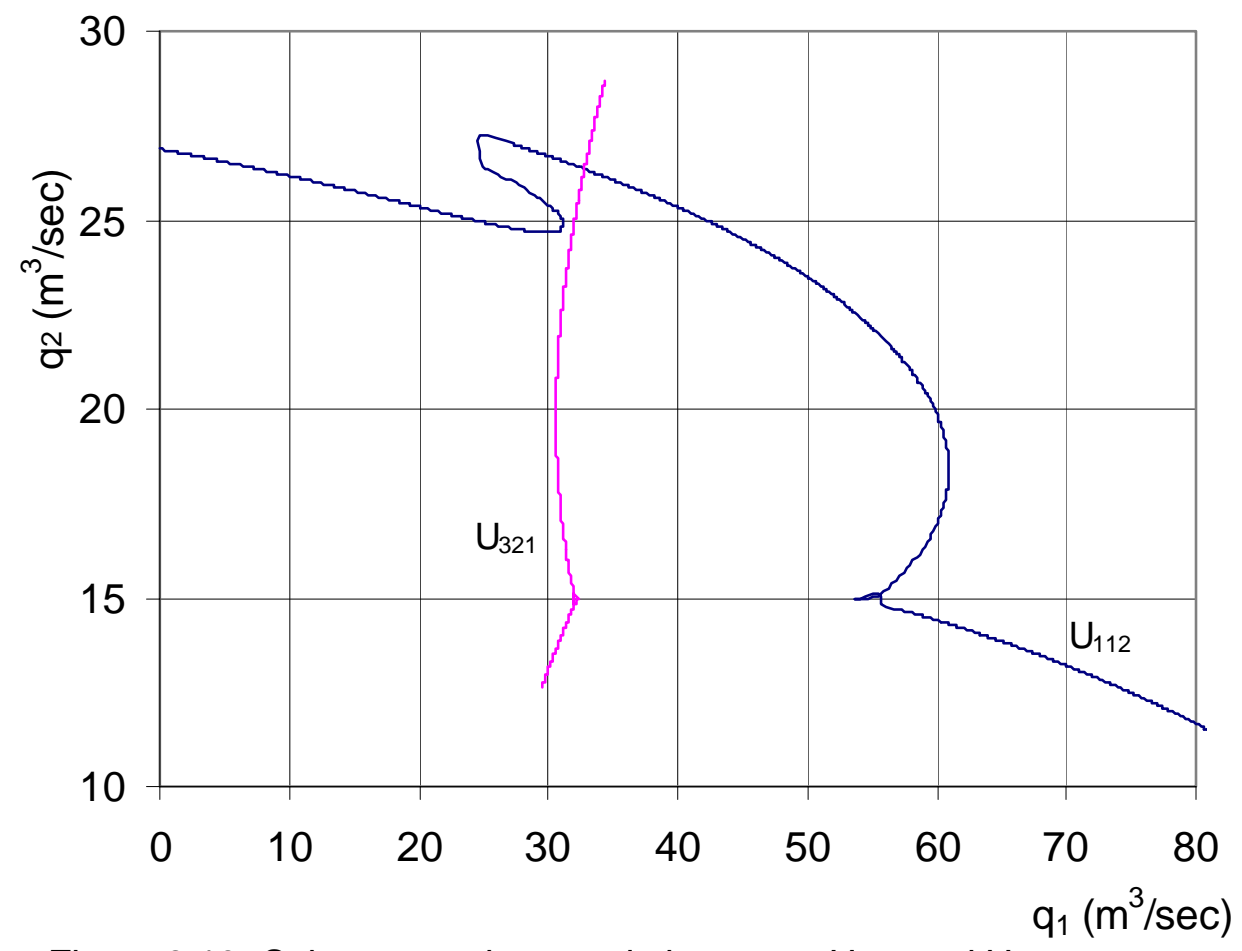

Figure 3.10 -Subsystem characteristic curves $U_{112}$ and $U_{312}$

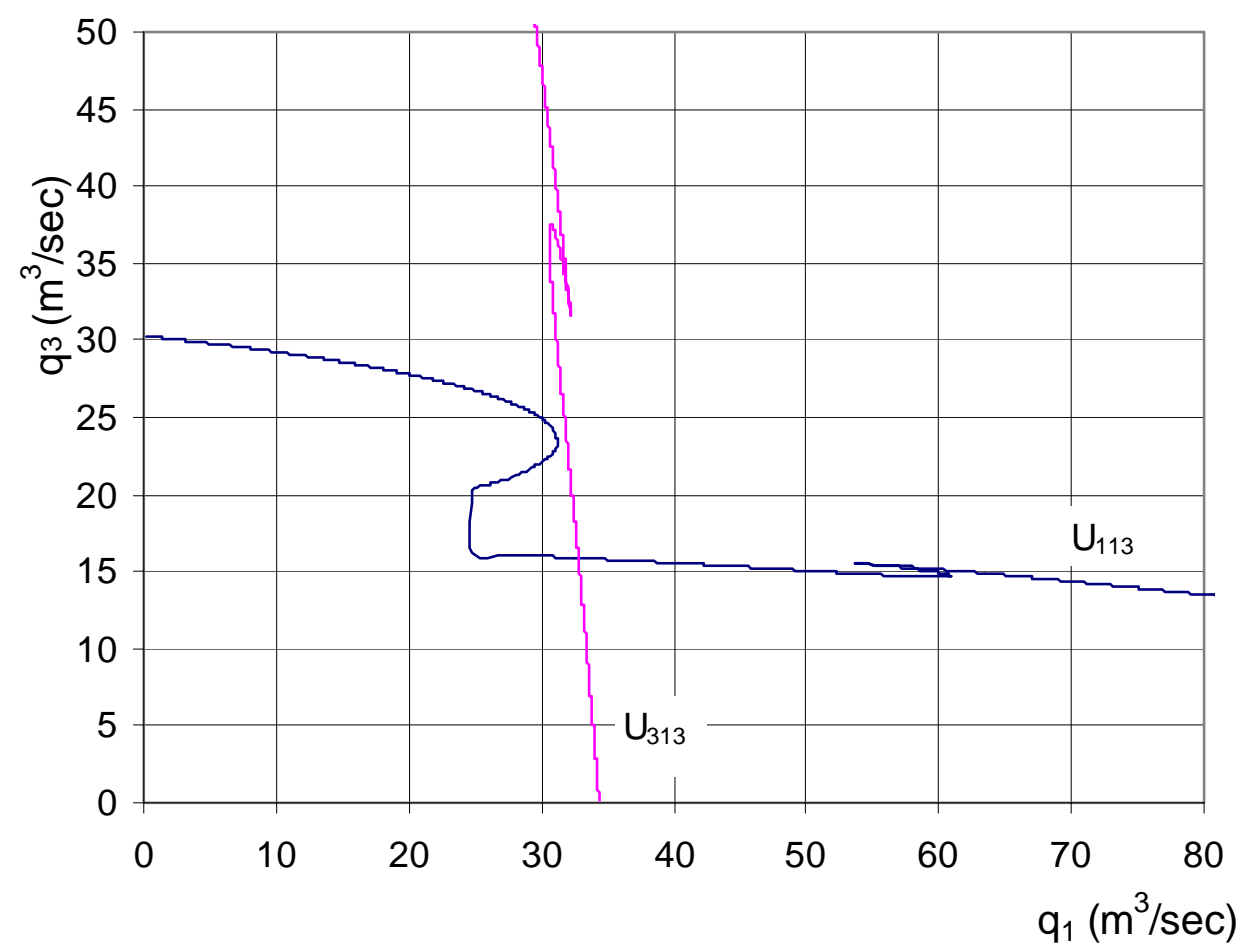

Figure 3.11 -Subsystem characteristic curves $U_{113}$ and $U_{313}$ 


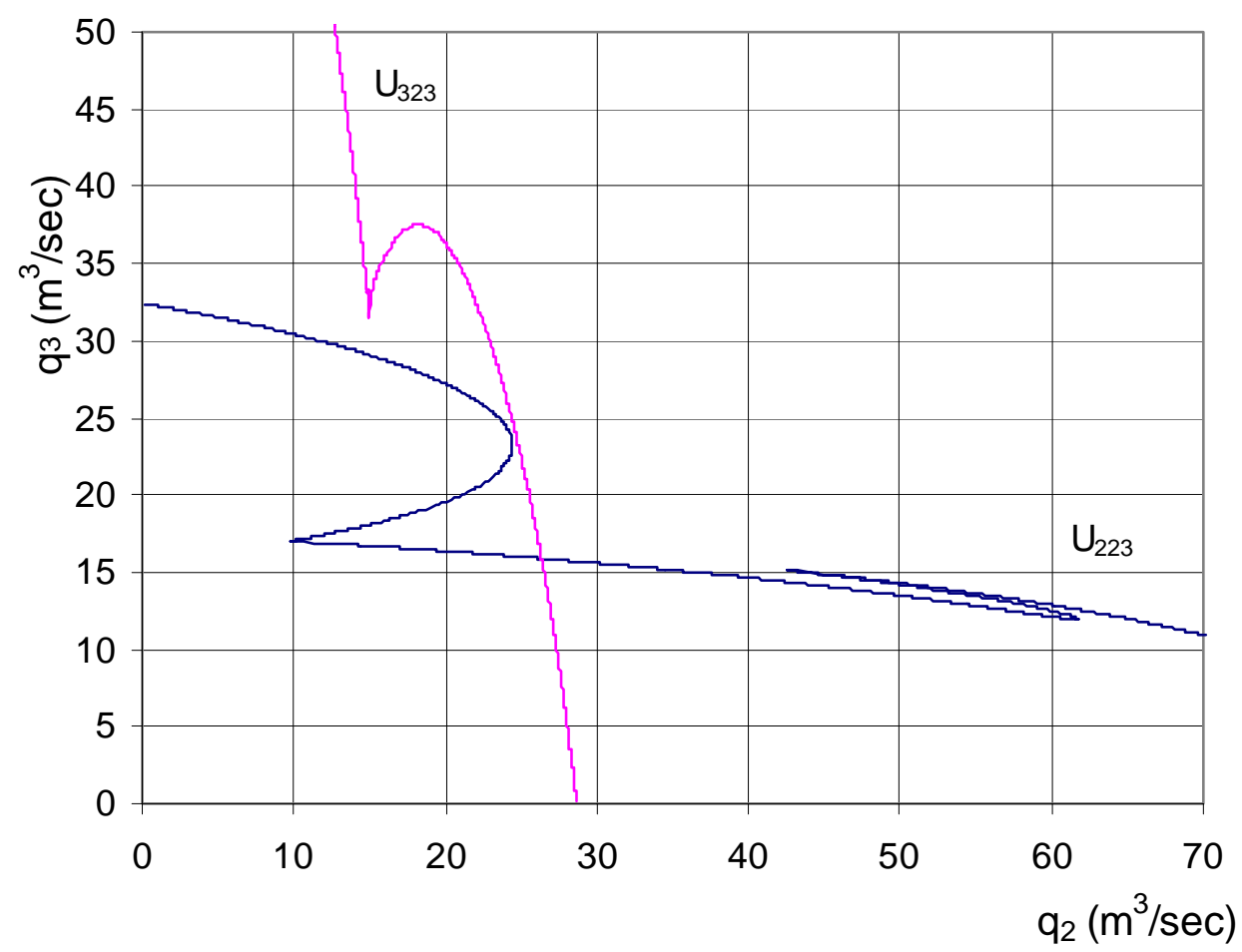

Figure 3.12 -Subsystem characteristic curves $\mathrm{U}_{223}$ and $\mathrm{U}_{323}$

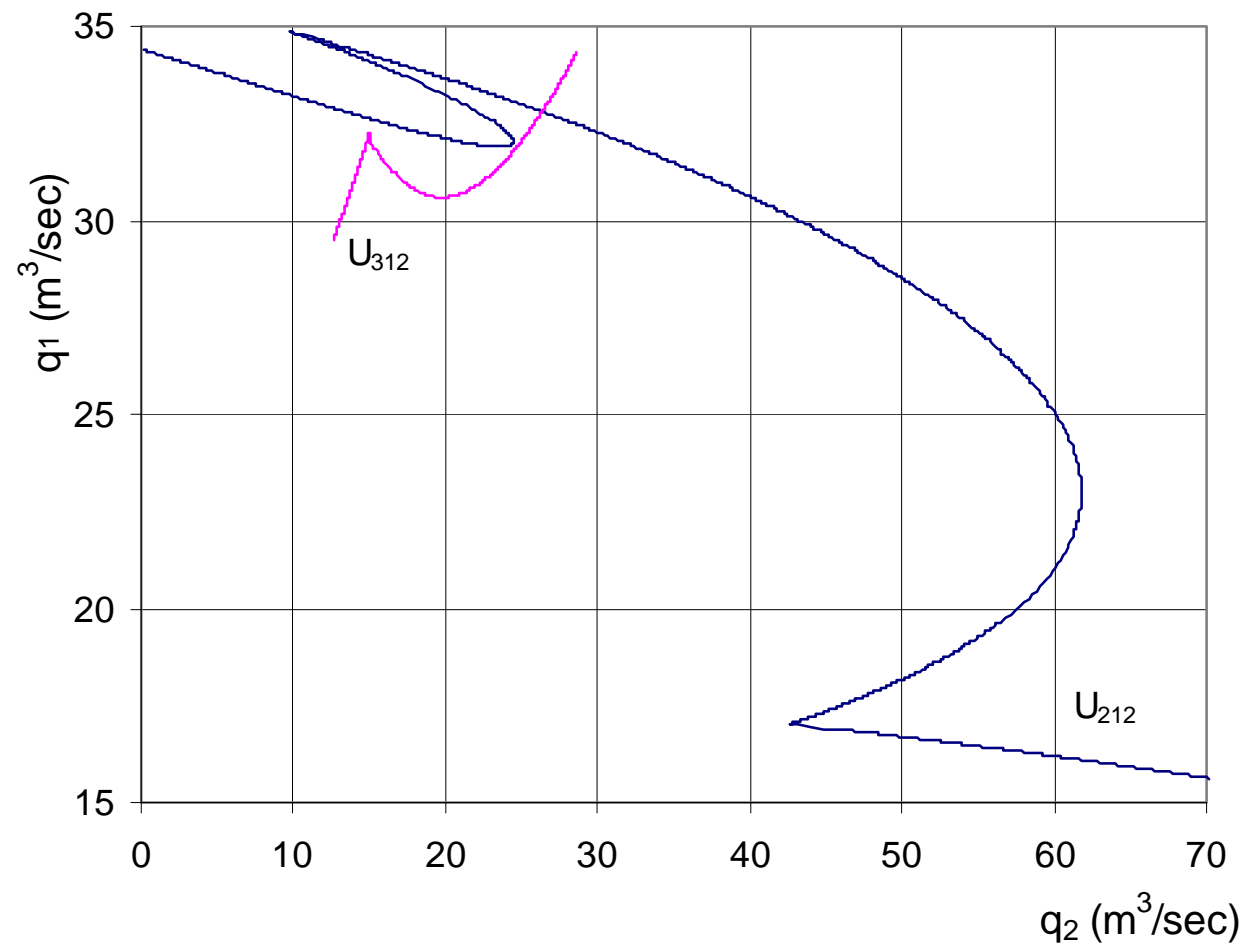

Figure 3.13 -Subsystem characteristic curves $U_{212}$ and $U_{312}$ 


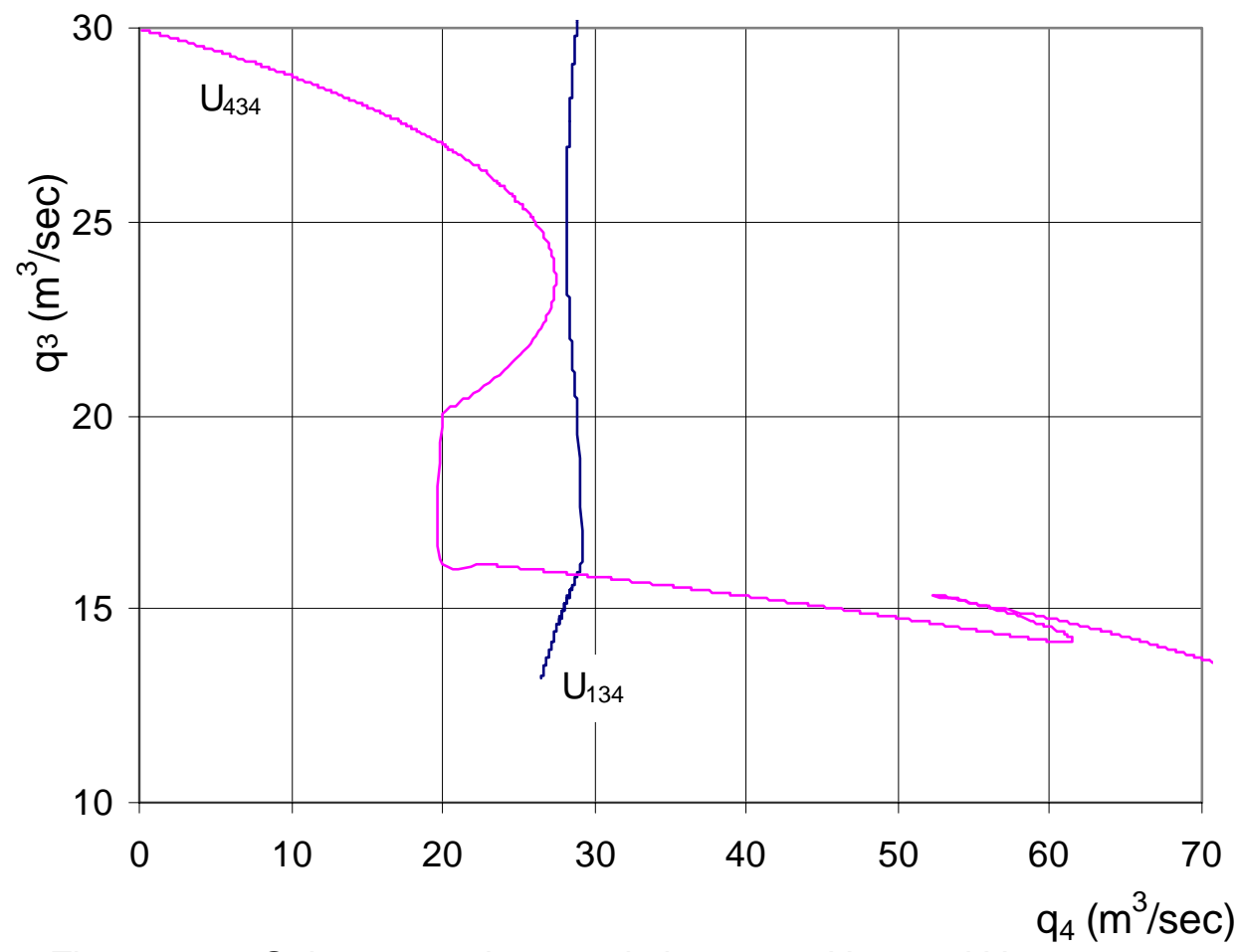

Figure 3.14 -Subsystem characteristic curves $U_{134}$ and $U_{434}$

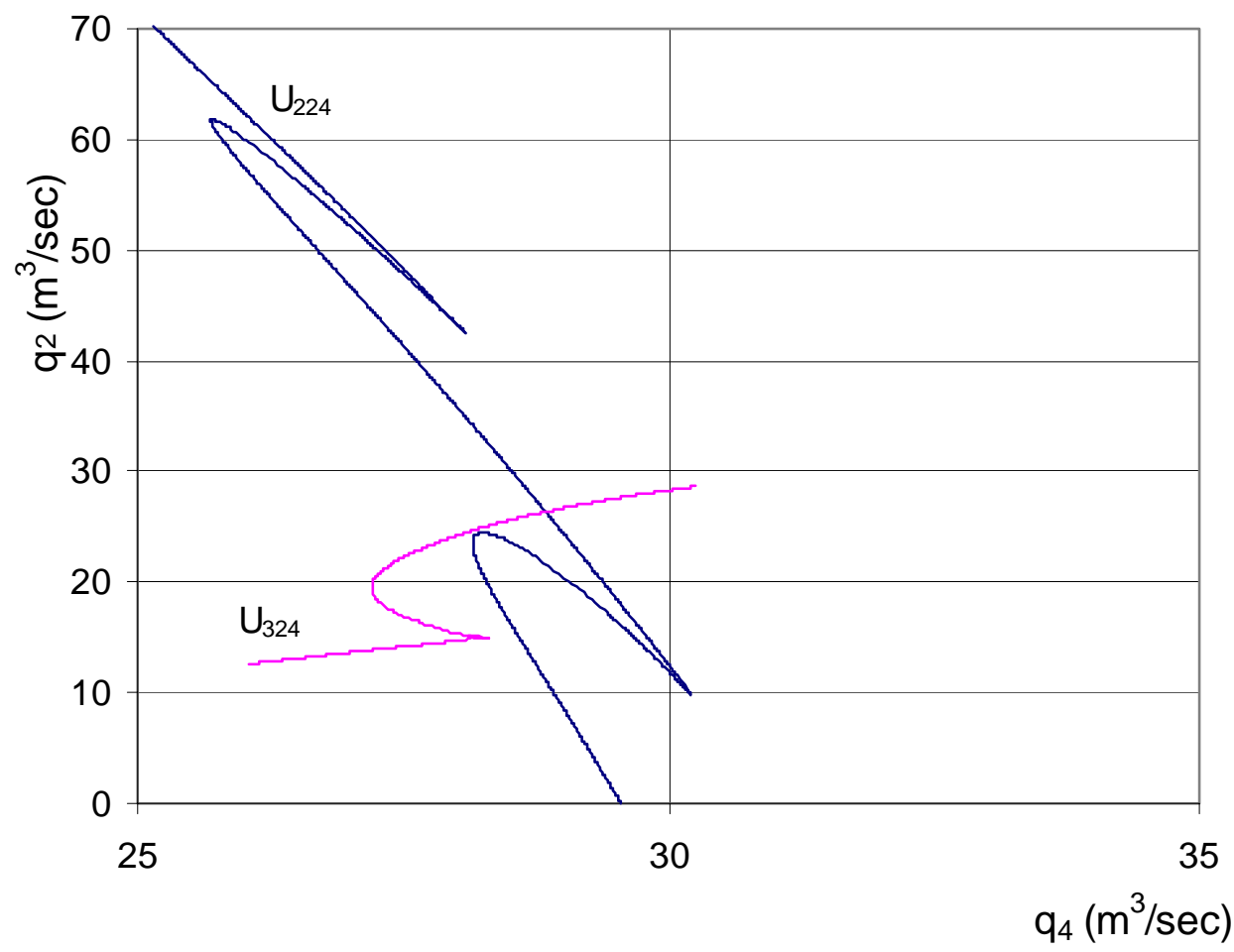

Figure 3.15 -Subsystem characteristic curves $\mathrm{U}_{224}$ and $\mathrm{U}_{324}$ 


\subsection{Relation Between Airflow and Subsystem's Resistance in Multiple Fan Systems}

The relation between airflow and the air quantity in a single fan system is a strictly increasing function that follows Atkinson's equation (Equation 2.3), while the resistance is treated as a constant. The subsystem characteristic curve in a multiple fan system does not have a fixed shape and no longer follows the normal path described by Atkinson's equation. To study the applicability of Atkinson's equation in multiple fan systems, the resistance factors have been calculated at each point of the subsystem curves 1, 2, 3 and 4. Resistance factors started very large at the small values of flow (at the investigated branch), and then dropped rapidly to a value near zero (Figures 3.6 to 3.9). At each subsystem when the reference delivers a small air quantity, the effect of the fans in the subsystem is enormous, and they work as extra resistance within the system. This effect fades away rapidly when the airflow in the subsystem reaches a reasonable value (around $10 \mathrm{~m}^{3} / \mathrm{sec}$ ). To study the statistical properties of the relationship between airflow and the pressure consumed at each subsystem, different statistical methods have been utilized to examine the data as shown in Table 3.2. $3^{\text {rd }}$ degree $\left(y=a+b * x+c * x^{2}+d *\right.$ $\left.\mathrm{x}^{3}\right)$ and quadratic fit $\left(\mathrm{y}=\mathrm{a}+\mathrm{b} * \mathrm{x}+\mathrm{c} * \mathrm{x}^{2}\right)$ polynomials models showed the best statistical analysis of all of the models used. The $2^{\text {nd }}$ order polynomial $\left(y=a+b * x^{2}\right)$, while (a) represents the intersection between the subsystem curve with y axis, presented a good statistical analysis too but the standard deviation is bigger than the standard deviation obtained from the $3^{\text {rd }}$ and quadratic fit models. Compared to the $2^{\text {nd }}$ order polynomial the power fit model $\left(y=a^{*} x^{b}\right)$ revealed bigger standard deviation while Atkinson's equation dedicated the worst standard deviation and correlation coefficient. 
Table 3.2 Standard deviation and correlation coefficient for some statistical models

\begin{tabular}{|c|c|c|c|c|c|c|c|}
\hline \multicolumn{8}{|c|}{$3^{\text {rd }}$ degree Polynomial Fit: $y=a+b * x+c * x^{2}+d * x^{3}$} \\
\hline Subsystem & a & $\mathrm{b}$ & \multicolumn{2}{|c|}{$\mathrm{c}$} & d & $\mathrm{S}$ & $\mathrm{r}$ \\
\hline 1 & 363.34162 & 18.741604 & \multicolumn{2}{|c|}{0.58301034} & 0.001001 & 35.25454 & 0.9997895 \\
\hline 2 & 816.49017 & 5.9453982 & \multicolumn{2}{|c|}{0.84738516} & 0.000391 & 84.60050 & 0.9979918 \\
\hline 3 & 676.50290 & 30.605574 & \multicolumn{2}{|c|}{0.08503650} & 0.007257 & 41.09479 & 0.9984637 \\
\hline 4 & 400.16527 & 15.088808 & \multicolumn{2}{|c|}{0.67344694} & 0.000451 & 31.89709 & 0.9999379 \\
\hline \multicolumn{8}{|c|}{ Quadratic Fit: $y=a+b * x+c * x^{2}$} \\
\hline Subsystem & $\mathrm{a}$ & $\mathrm{b}$ & \multicolumn{2}{|r|}{$\mathrm{c}$} & \multicolumn{2}{|r|}{$\mathrm{S}$} & $\mathrm{r}$ \\
\hline 1 & 394.40313 & 14.518420 & \multicolumn{2}{|c|}{0.70808619} & \multicolumn{2}{|c|}{36.9276067} & 0.9997688 \\
\hline 2 & 825.24121 & 4.7163666 & \multicolumn{2}{|c|}{0.88888621} & \multicolumn{2}{|c|}{84.6007615} & 0.9979902 \\
\hline 3 & 732.19565 & 18.26591 & & 6587705 & 45.5 & 738632 & 0.9981071 \\
\hline 4 & 432.21372 & 11.80396 & & 74766854 & 33.6 & 145689 & 0.9999310 \\
\hline & & Pow & r Fit: J & $=a^{*} x^{b}$ & & & \\
\hline Subsystem & $\mathrm{a}$ & $\mathrm{b}$ & & & $\mathrm{S}$ & & $\mathrm{r}$ \\
\hline 1 & 10.820807 & 1.438 & 2647 & 169. & .6156455 & & 951053 \\
\hline 2 & 43.716317 & 1.114 & 400 & 263. & .5791872 & & 803020 \\
\hline 3 & 150.77957 & 0.762 & 3448 & 202.8 & .8652372 & & 617339 \\
\hline 4 & 5.4713534 & 1.607 & 7004 & 206.3 & .3826232 & 0.9 & 973939 \\
\hline & & $2^{\text {nd }}$ order p & lynomi & al $\mathbf{y}=\mathbf{a}+$ & $-b^{*} x^{2}$ & & \\
\hline Subsystem & $\mathrm{a}$ & $\mathrm{b}$ & & & $\mathrm{S}$ & & $\mathrm{r}$ \\
\hline 1 & 631.72607 & 0.871 & 2888 & 90.6 & 6690884 & 0.9 & 986038 \\
\hline 2 & 888.65643 & 0.952 & 2739 & 86.7 & 7710420 & & 978840 \\
\hline 3 & 912.81687 & 0.995 & 2988 & 79.9 & 9213688 & & 941574 \\
\hline 4 & 682.50512 & 0.848 & 9469 & 96.1 & 1290645 & 0.9 & 994352 \\
\hline & & Atkinson & Equa & tion $\mathrm{y}=\mathbf{a}$ & ${ }^{*} * \mathrm{x}^{2}$ & & \\
\hline Subsystem & & $\mathrm{a}$ & & $\mathrm{S}$ & & & $r$ \\
\hline 1 & 1.025 & 99075 & & 419.84408 & 837 & 0.96 & 95917 \\
\hline 2 & 1.26 & 50068 & & 572.84918 & 880 & 0.90 & 31092 \\
\hline 3 & $1.59^{\prime}$ & 79755 & & 573.07036 & 617 & 0.63 & 24254 \\
\hline 4 & 0.950 & 001984 & & 470.96949 & 967 & 0.98 & 63423 \\
\hline
\end{tabular}




\subsection{Comparison Between the Performance of Hardy Cross and Damped Newton-Raphson Method}

The Newton-Raphosn method is an efficient tool to solve systems of nonlinear equations once the initial guess is close enough to the solution point. As has been discussed before, the regular Newton-Raphson is not efficient enough to trace a subsystem in multiple fan systems, especially those that have a sharp turning points. The damped Newton-Raphson method, with a proper damped value, performs this task easily (Equation 3.11). Even better tracing efficiency would be obtained if the damped value in the Newton-Raphson method is smaller but the convergence rate would become very slow (Conte and Carl de Boor, 1980).

As shown in Figures 3.6 to 3.15 the subsystem characteristic curves have very sharp turning points. Thus a value of 0.2 of damped values was chosen and found to be adequate enough to trace the subsystem curves. The damped Newton-Raphson method is very slow compared to the Hardy Cross method. It takes up to 20 minutes to calculate all solution points and obtain the subsystem characteristic curve, while the Hardy Cross method takes about 5 minute to perform the same task on a relatively simple system.

\subsection{Summary}

Mathematical models that solve nonlinear equations cannot directly be applied to trace the subsystems in multiple fan systems. The switching parameters algorithm is a good tool for tracing subsystem characteristic curves. In the switching parameters, the independent parameter is not fixed and is chosen at each iteration step. The subsystem pressure drop $\mathrm{P}_{\mathrm{k}}$ is treated as a dependent parameter and air flow in the chords has been 
chosen as the independent parameter. The independent parameters in multiple fan systems have been chosen as the airflow in a chord that has the largest rate of change in the previous step. The independent airflow chord was treated as a fixed quantity branch. Extra fans or regulators are not allowed in the current method of multiple fan system analysis, so the cutset and cutset operation techniques have been used to redistribute the airflow across the network to keep the air flow fixed though the fixed quantity branch.

Modified Hardy Cross and damped Newton-Raphson methods implemented by the switching parameters algorithm are found to be good tools for solving multiple fan systems. The implemented Hardy Cross method has a higher convergence rate than the damped Newton-Raphson method. The shape of subsystem characteristic curves in a multiple fan system does not have a fixed shape and does not follow Atkinson's equation. 


\section{CHAPTER 4 \\ OPERATING CHARACTERISTICS OF MULTIPLE FAN SYSTEMS}

\subsection{Introduction}

In his dissertation, (Lin, 1995) used the implemented Quasi-Newton method to investigate the effect of some of the parameters on the operating characteristics of multiple-fan systems. He studied the influence of branch resistance changes, the influence of fan characteristics, the influence of a system's structural (topology) configuration, and the influence of the number of axial-flow fans. The writer concluded that the system's structural configuration does not have any effect on the shape of the subsystem characteristic curve. Changing the resistance factors of any branch cannot eliminate the non-monotonic subsystem-operating characteristic, but it is possible to incorporate the characteristics of the fans to achieve a single system operating point.

This chapter reviews some of the parameters that have been studied before and continues the investigation into the parameters that affecte the shape of the subsystem characteristic curve and the number of the operating points in the system and consequently, the stability of the system. These parameters include the effect of the resistance factor, the effect of the number of branches and meshes (or the topology of the network), the number of fans, the types of fans in the subsystem, and the effect of the fans operating zones inside the subsystem on the shape of the subsystem characteristic curve. 


\subsection{Effect of Resistance Factors on the Shape of the Subsystem Characteristic Curve}

In mine ventilation networks, branches are classified into two types; fan branches and non-fan or regular branches. In a single fan system, increasing the resistance of any branch in the network increases the overall resistance of the system and consequently increases the negative slope of the mine characteristic curve, regardless the type of the branch.

\subsubsection{Impact of the Fan Branch's Resistance Changes on the Shape of the Subsystem Characteristic Curve}

To study the effect of changing the fan branch resistance on the shape of the subsystem characteristic curves, the resistance factor of branch No. 1 (Figure 3.4) has been changed from 0.7 to 0.3 and then to $2.0 \mathrm{~N} . \mathrm{s}^{2} / \mathrm{m}^{8}$. As shown in Figure 4.1, the effect of changing the fan branch's resistance on the shape of its associated fan subsystem is as follows:

- The effect is very small at small airflow quantities that moving through the associated branch. Subsystem 1 characteristic curve at a resistance $\mathrm{r} 1=0.3,0.7$ and $2.0 \mathrm{~N} . \mathrm{s}^{2} / \mathrm{m}^{8}$ starts at the same point; zero airflow of branch 1, and then spreads away at higher airflows.

- At high airflow, as in a single fan system, the larger the resistance the higher the slope of the subsystem characteristic curve. 
This effect on the shape is not the same as that of the other subsystems. Figures 4.2 to 4.4 show the effect of changing the resistance of branch No. 1 on the shape of the subsystems characteristic curves of fans 2,3 and 4 respectively. This effect can be summarized as follows:

- The subsystem characteristic curves do not start from the same point at different values of resistances in branch No. 1. This indicates that changing the fan branch's resistance has an effect on all the other fans subsystem curves in the system.

- Unlike single fan systems, if a fan's branch resistance increases, the other fan's subsystem characteristic curve moves downward, and vice versa.

- The number, length and the locations of the zigzag portion of the subsystem characteristic curves are varied and they may shift with the changing of a fan branch resistance in another branch.

- Changing the resistance of the fan branch can control the number of operating points in the system. In the example there is only one operating point of the system when $\mathrm{r} 1=0.3 \mathrm{~N} . \mathrm{s}^{2} / \mathrm{m}^{8}$ and there are three operating points when it was 2.0 N. $\mathrm{s}^{2} / \mathrm{m}^{8}$. 


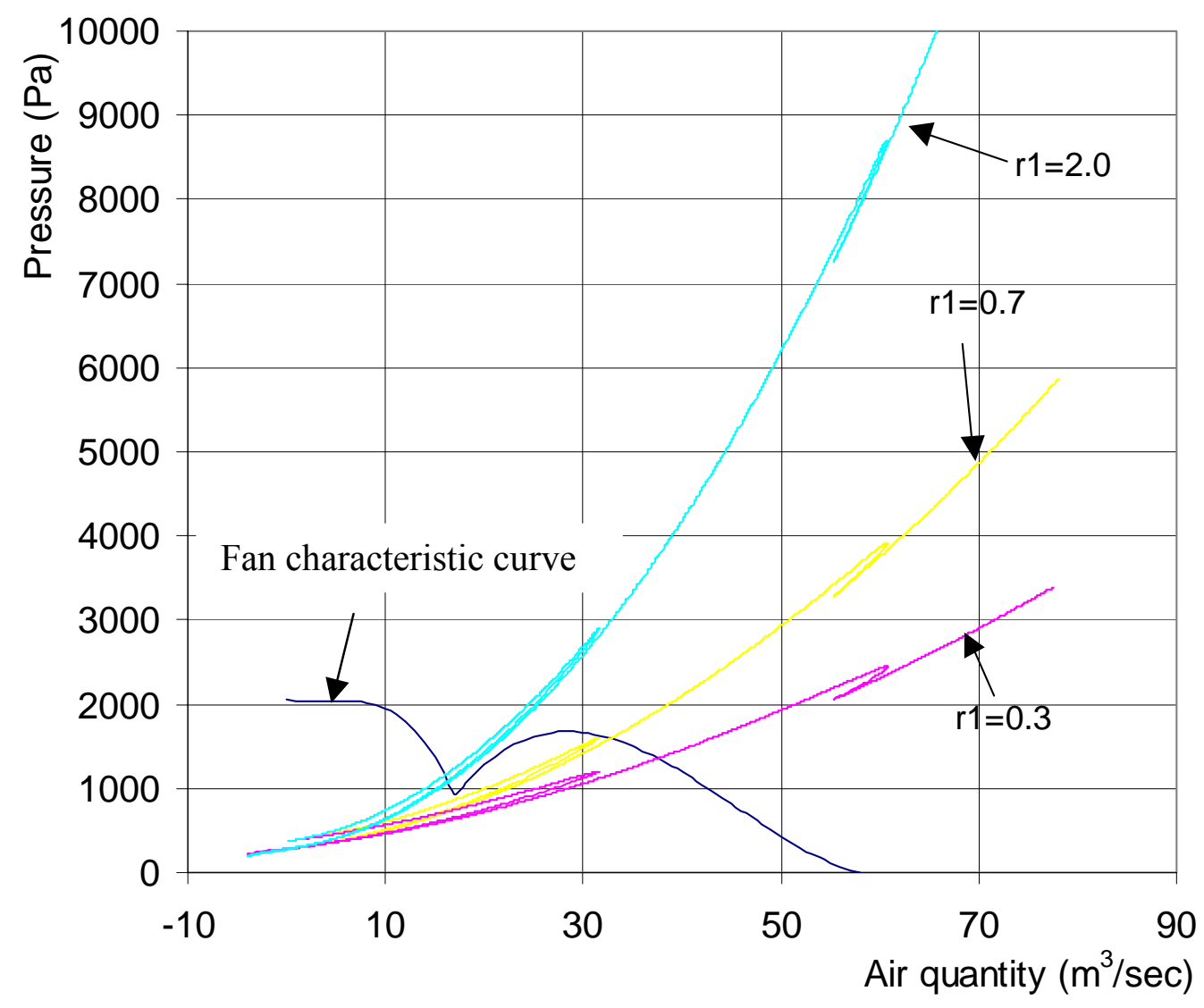

Figure 4.1 -Effect of changing the resistance of fan branch No. 1 on the shape of its subsystem characteristic curve. 


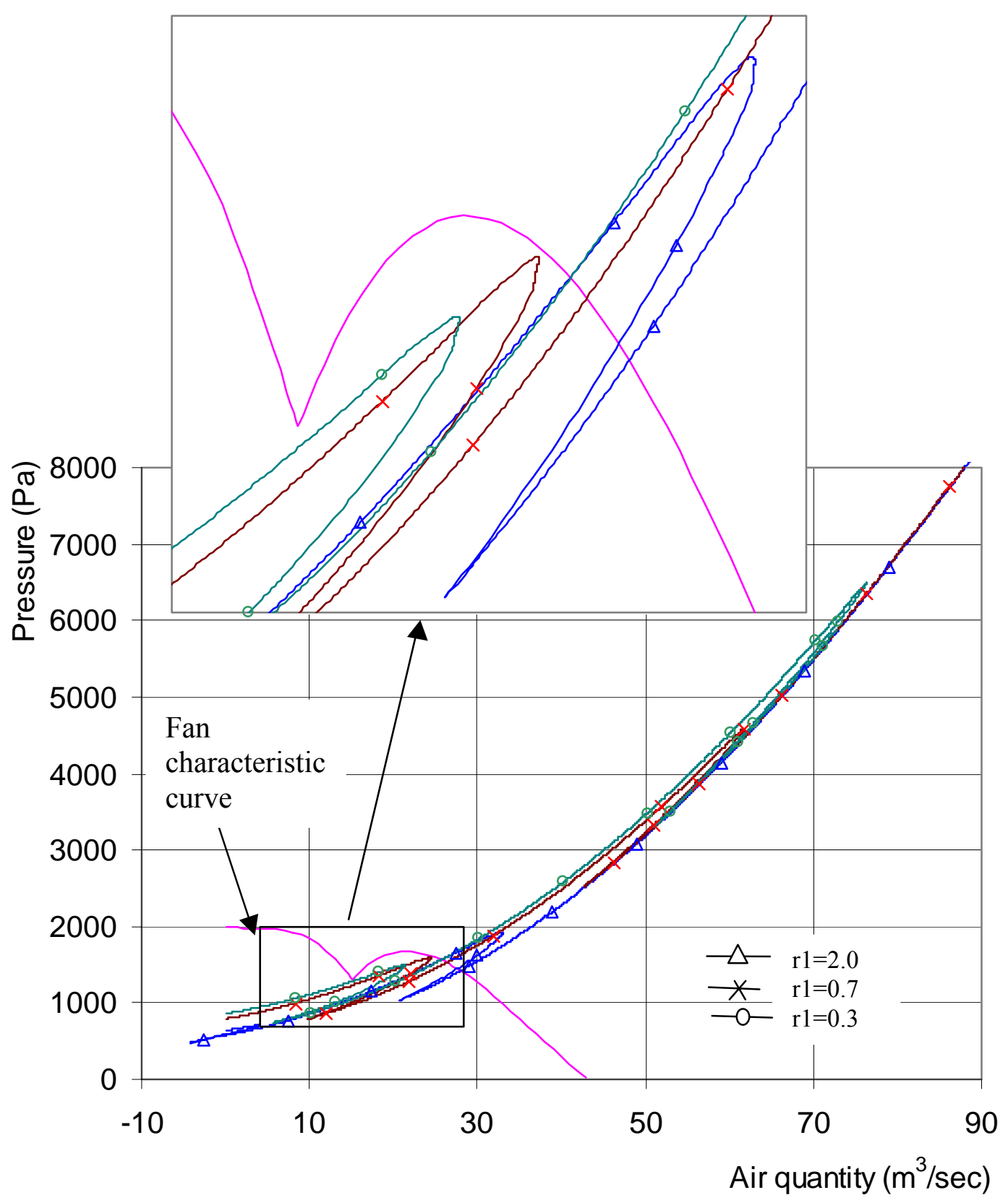

Figure 4.2 -Effect of changing the resistance of fan branch No. 1 on the shape of subsystem No. 2 characteristic curve. 


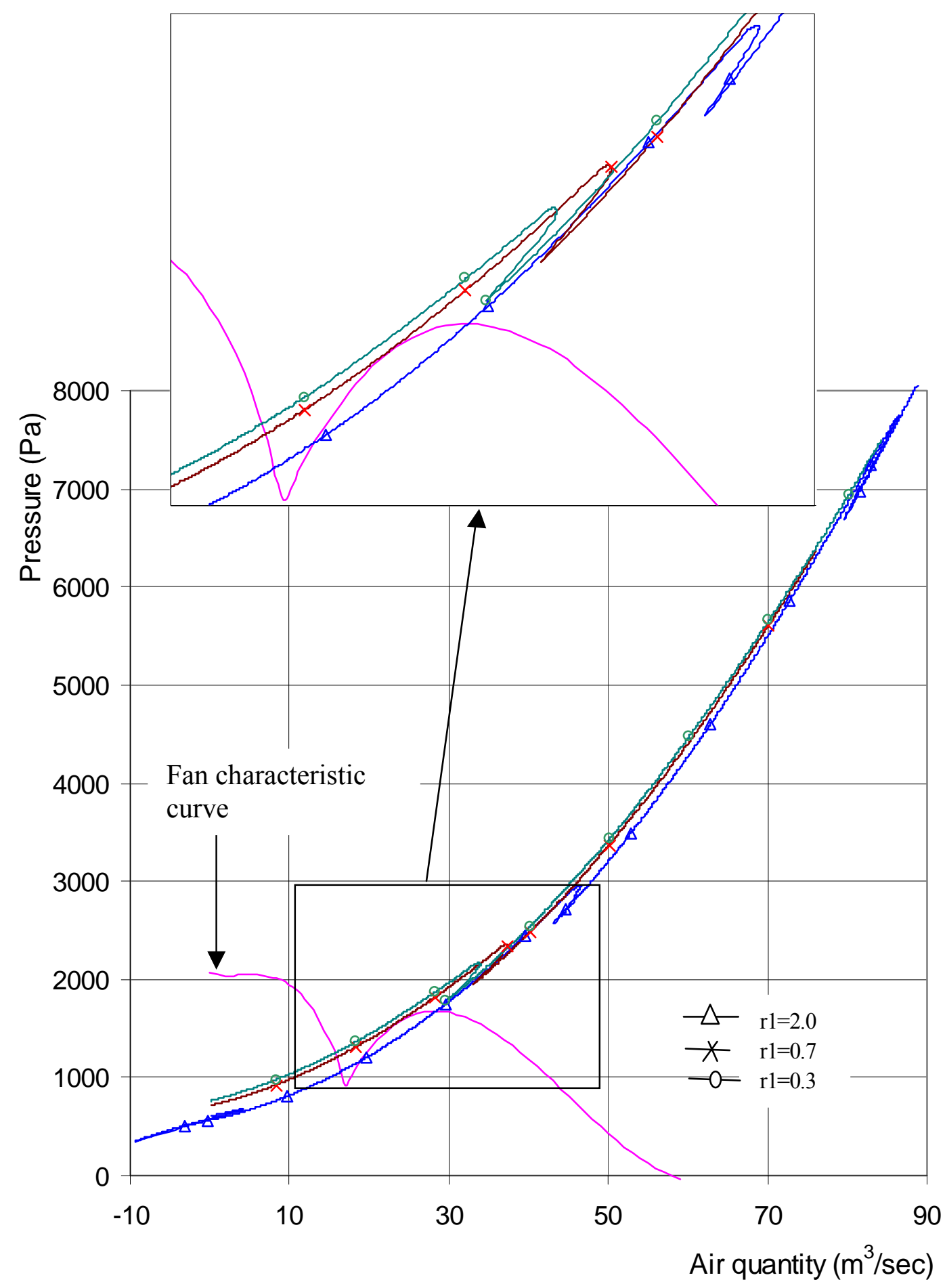

Figure 4.3 -Effect of changing the resistance of fan branch No. 1 on the shape of subsystem No. 3 characteristic curve. 


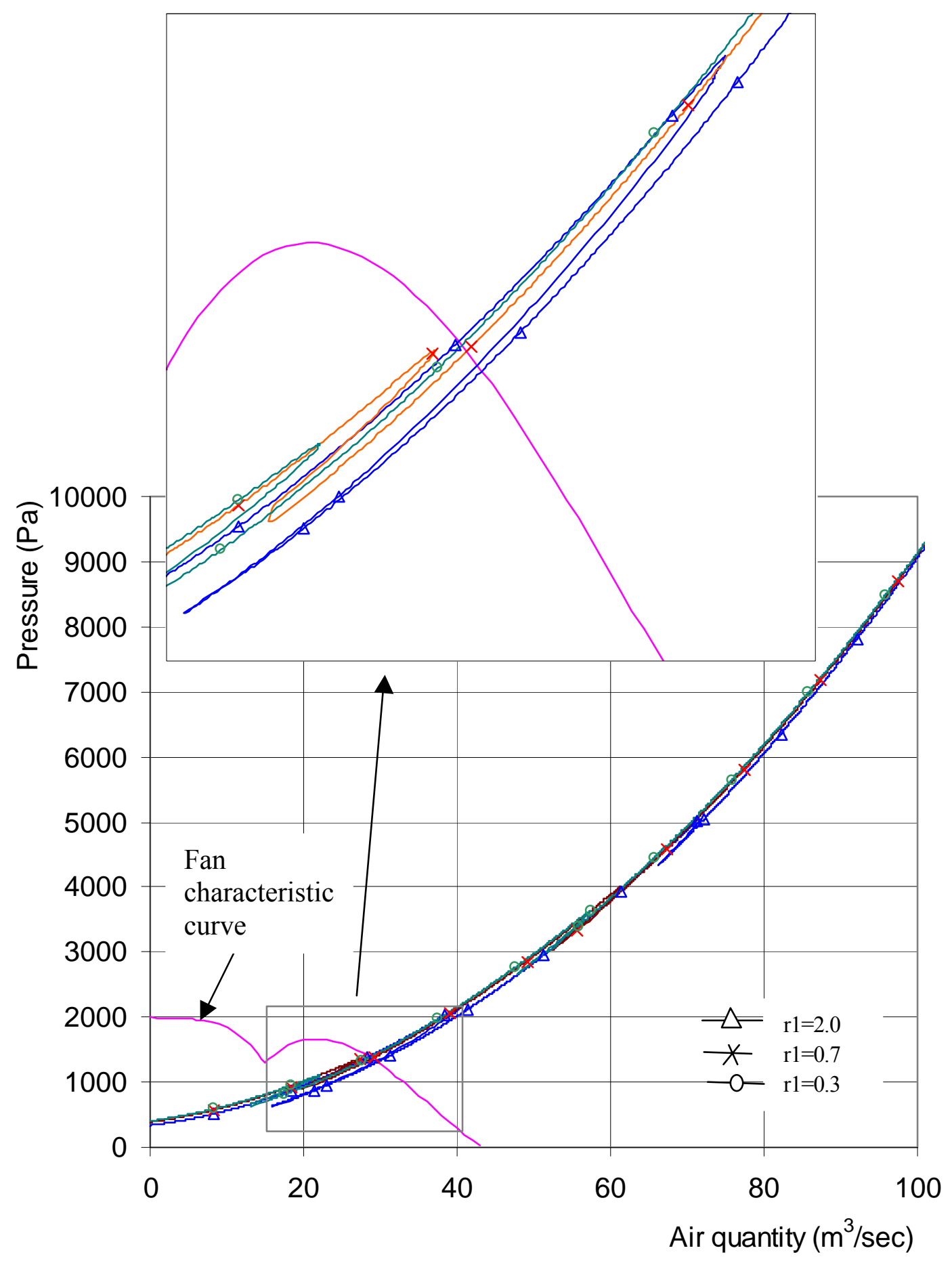

Figure 4.4-Effect of changing the resistance of fan branch No. 1 on the shape of subsystem No. 4 characteristic curve. 


\subsubsection{Impact of the Regular Branch's Resistance Changes on the Shape of the Subsystem Characteristic Curve}

In real mine ventilation networks, changing the resistance of non-fan branches by using regulators or extra auxiliary fans is very common. Figures 4.5 to 4.8 show the effect of changing the resistance factor in branch No. 9 in Figure 3.4, from $0.3 \mathrm{~N} . \mathrm{s}^{2} / \mathrm{sec}^{8}$ to $0.3 / 100$ and then to $0.3 * 300 \% \mathrm{~N} \cdot \mathrm{s}^{2} / \mathrm{sec}^{8}$, on the shape of the subsystem curves $1,2,3$ and 4 respectively. While decreasing the regular branch resistance, the subsystem pressure decreases. This effect is not quantitatively constant. The effect of changing the resistance of a non-fan branch on the shape of the subsystem curves depends upon the topology of the network. This effect is very large and distinct upon the shape of the subsystem characteristic curve No. 4. The effect decreases in the order subsystem 3, subsystem 2, and subsystem 1 . Changing a regular branch's resistance effects not only the subsystem pressure, but also the location and the space among the zigzag parts of the curve. Increasing the regular branch's resistance not only moves the zigzag part toward the larger airflow, but also makes these zigzag parts closer. As shown in Figure 4.7, the undesirable condition of fan No. 3, working on it unstable zone, can be avoided by decreasing the resistance at branch No. 9 from 0.3 to $0.3 / 100 \mathrm{~N} \mathrm{~s}^{2} / \mathrm{sec}^{8}$. This effect is very clear, not only on the dip of subsystem No. 4, but also on the number of zigzagging parts of the curve. Figure 4.8 shows the resistance factor of branch No. 9 equal to $0.3 / 100$ $\mathrm{N} \mathrm{s}^{2} / \mathrm{sec}^{8}$. The shape of the subsystem characteristic curve is the same as a single fan system as it passes through the origin point and there is no zigzag part on the curve. 


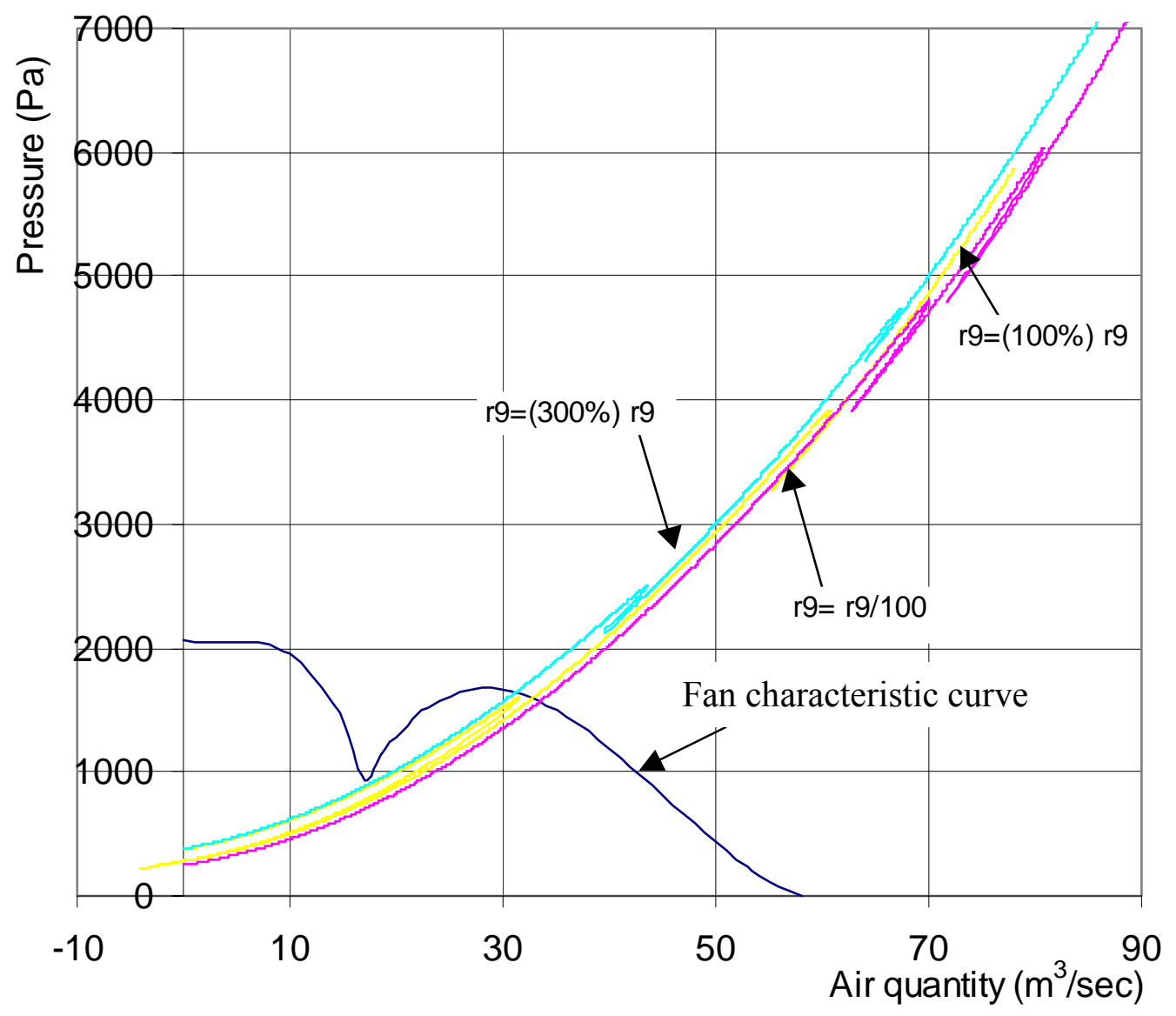

Figure 4.5 -Effect of changing the resistance of regular branch No. 9 on the shape of subsystem No.1 characteristic curve. 


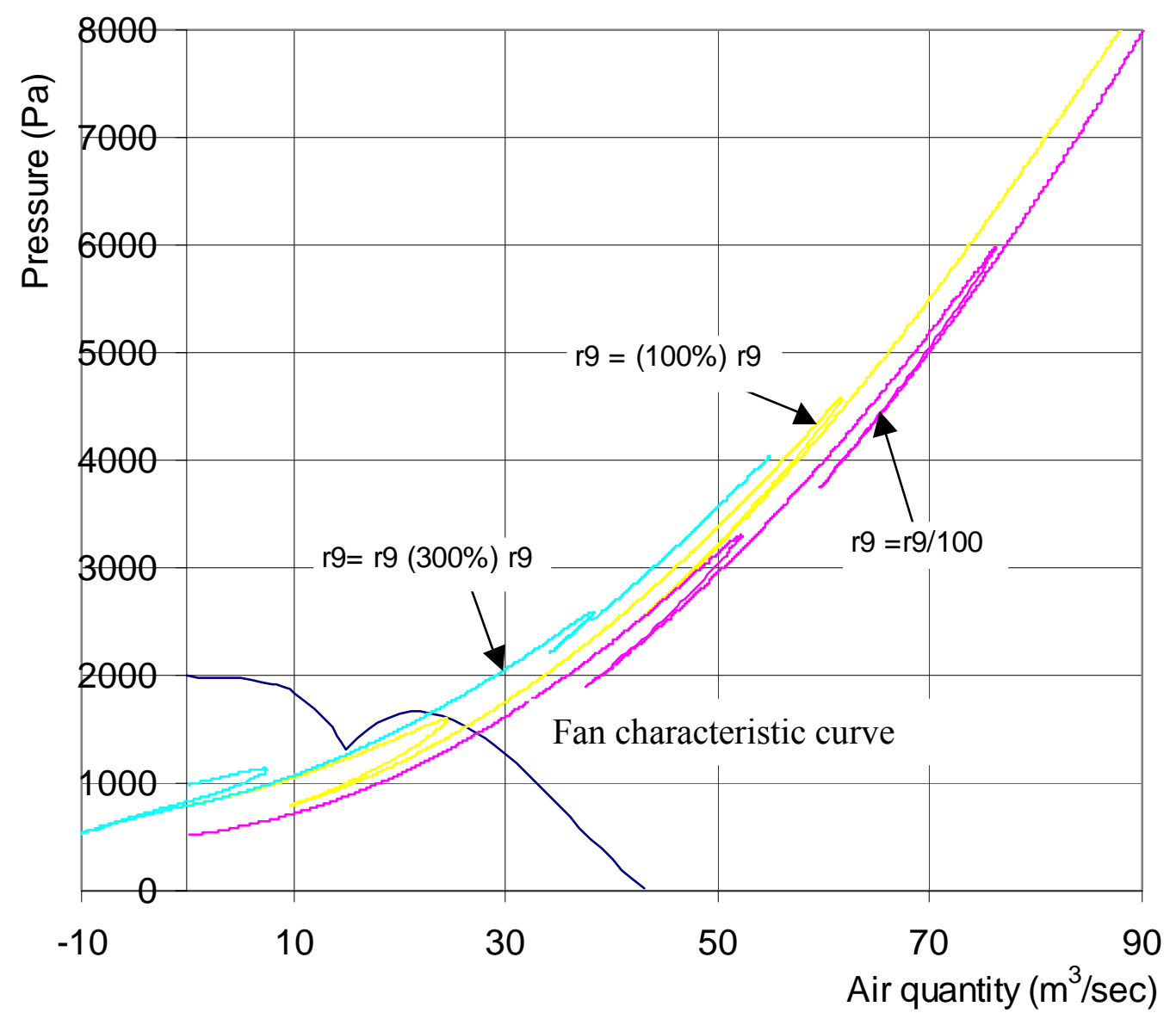

Figure 4.6-Effect of changing the resistance of regular branch No. 9 on the shape of subsystem No. 2 characteristic curve. 


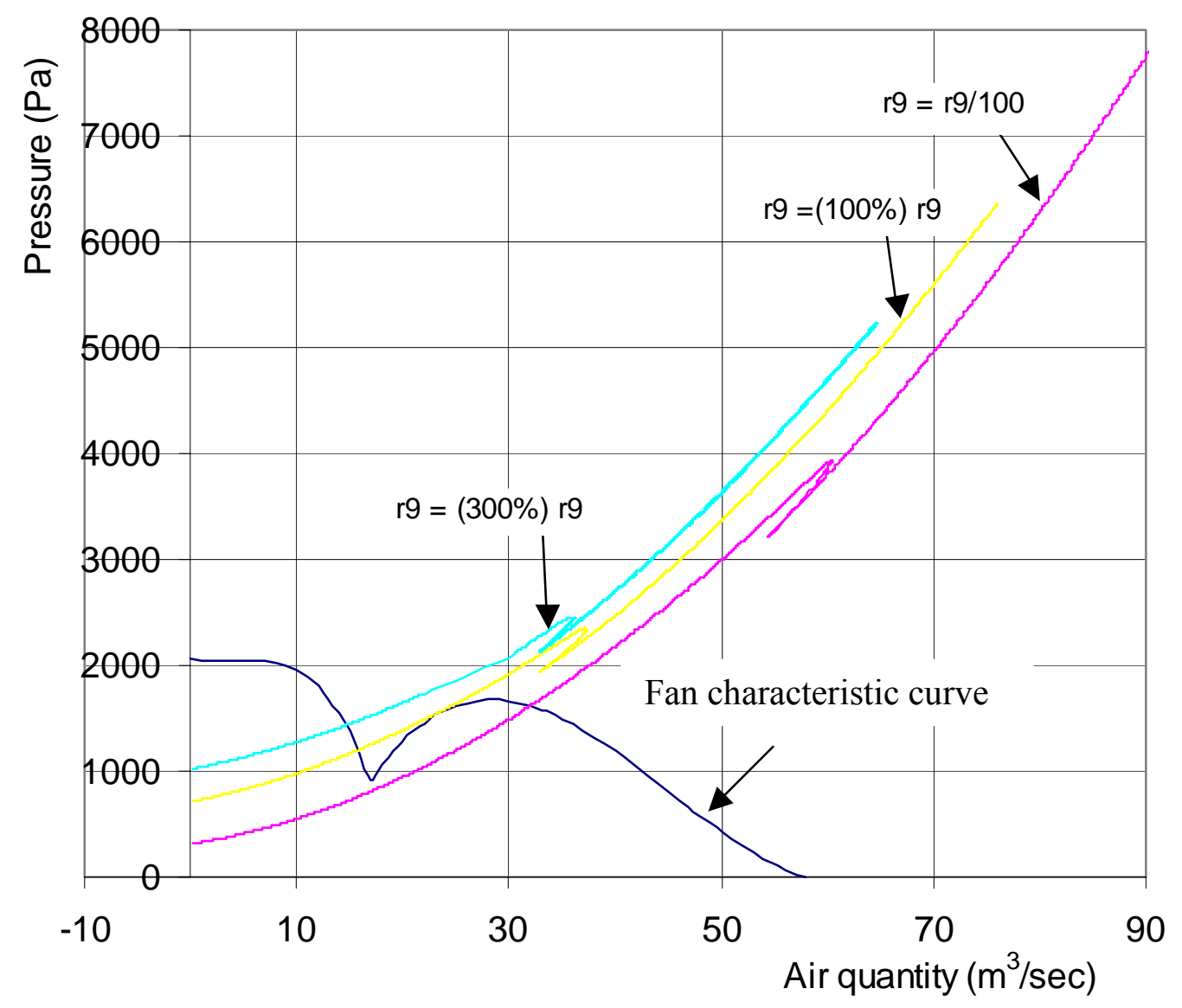

Figure 4.7 -Effect of changing the resistance of regular branch No. 9 on the shape of subsystem No. 3 characteristic curve. 


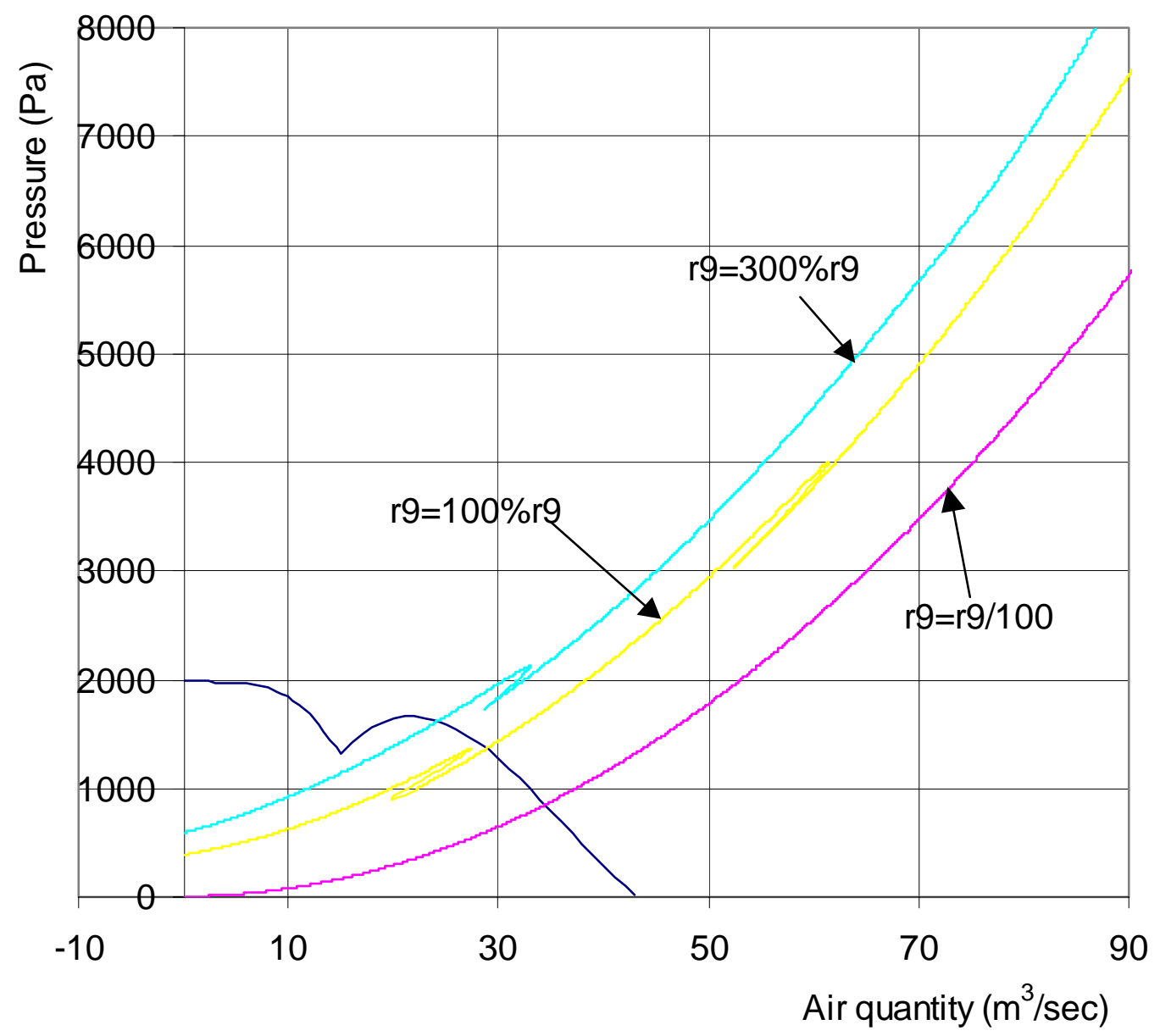

Figure 4.8 -Effect of changing the resistance of regular branch No. 9 on the shape of subsystem No. 4 characteristic curve. 


\subsection{Effect of the Topology of the Network on the Shape of the Subsystem Characteristic Curve}

In mine ventilation networks, increasing the number of parallel connections decreases the over all resistance of the network, consequently decreasing the slope of the mine characteristic curve. Increasing the number of series connections increases the over-all resistance of the system. Generally speaking, increasing the number of openings in the network decreases the resistance.

To study the effect of changing the topology of the network on the shape of the subsystem characteristic curves, three networks have been studied. Each of these networks has four axial flow fans installed in their first 4 branches. The first network consists of 5 meshes, 6 nodes and 10 resistances (Figure 3.4). The second network, Figure 4.9, consists of 8 meshes 9 nodes and 16 branches. The third network, Figure 4.10, has 16 meshes, 14 nodes and 29 branches. The resistance factors of the branches are indicated in order of the capture of each figure.

As shown in Figures 4.11 to 4.14 , the effect of changing the topology of the network is considerable in the shape of the subsystem characteristic curves. The zigzag parts in example Figure 3.4 have entirely vanished. The subsystem characteristic curves in the multiple fan system moves toward the common mine characteristic curve of a single fan system. The over all resistance of the subsystem, and consequently the slope of the subsystem, decrease, which then decreases the number of operating points if the number of branches and meshes in the network is increased. As a result, most of the fans in the multiple fan systems work within their stable zones. Subsystem characteristic 
curves pass close to origin by increasing the openings in the network. From the above discussion, we can conclude that the effect of fans inside any subsystem decreases by increasing the number of branches and meshes in the network, and accordingly the multiple fan system becomes more stable. By adding so many branches and nodes, the system has basically becomes four independent single fan systems that are connected together by very low resistance pathways. 


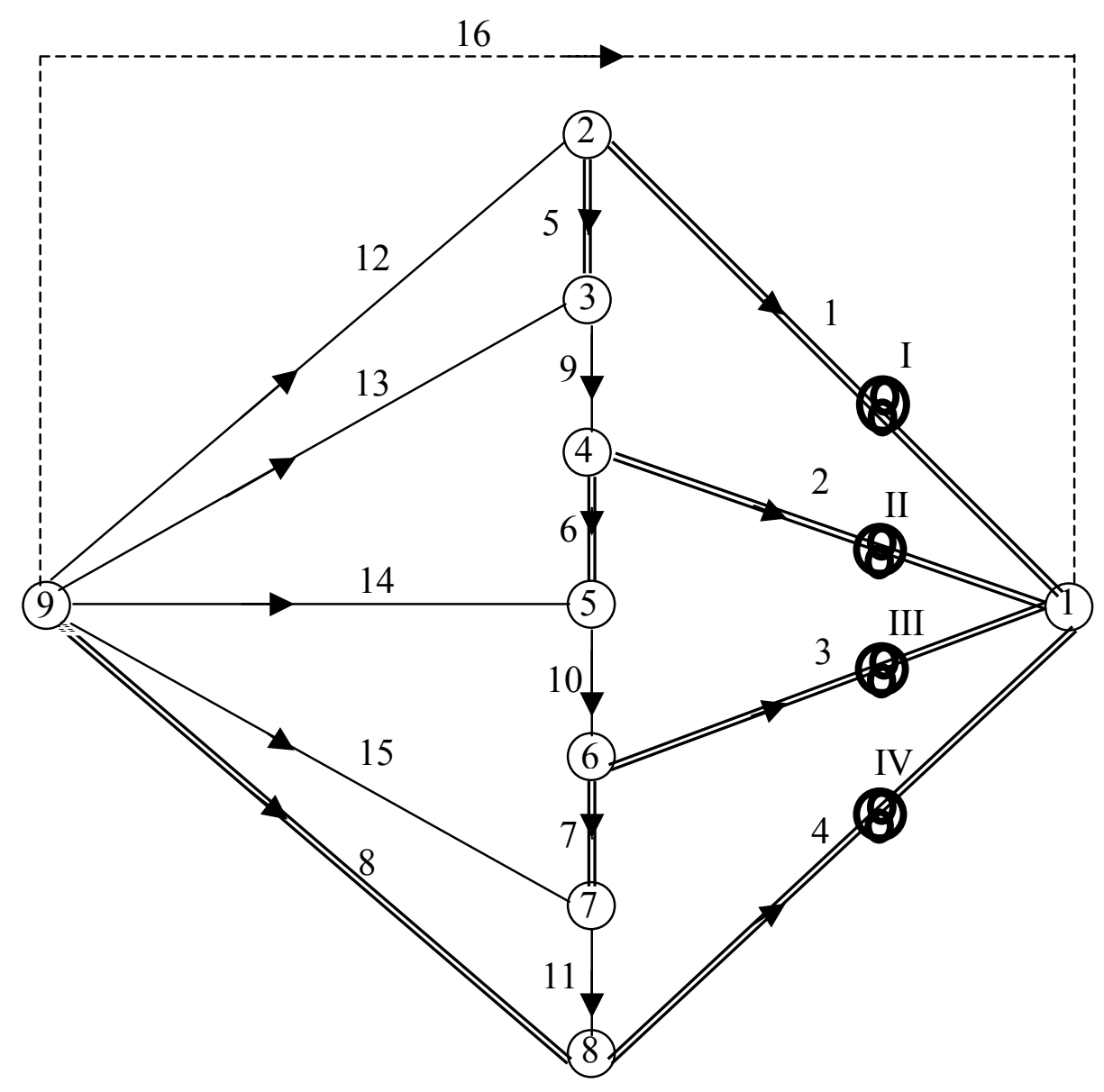

Figure 4.9 -The topology of the 8 meshes, 9 nodes and 16 branches network. The resistance factors in N.s $/ \mathrm{m}$ are shown in the table below.

\begin{tabular}{|c|c||c|c|}
\hline Branch No. & $\begin{array}{c}\text { Resistance } \\
\text { N.s }{ }^{2} / \mathrm{m}^{8}\end{array}$ & Branch No. & $\begin{array}{c}\text { Resistance } \\
\mathrm{N} . \mathrm{s}^{2} / \mathrm{m}^{8}\end{array}$ \\
\hline 1 & 0.7 & 9 & 0.4 \\
\hline 2 & 0.7 & 10 & 0.4 \\
\hline 3 & 0.7 & 11 & 0.4 \\
\hline 4 & 0.7 & 12 & 0.3 \\
\hline 5 & 0.5 & 13 & 0.3 \\
\hline 6 & 0.5 & 14 & 0.3 \\
\hline 7 & 0.5 & 15 & 0.3 \\
\hline 8 & 0.3 & 16 & 0.0 \\
\hline
\end{tabular}




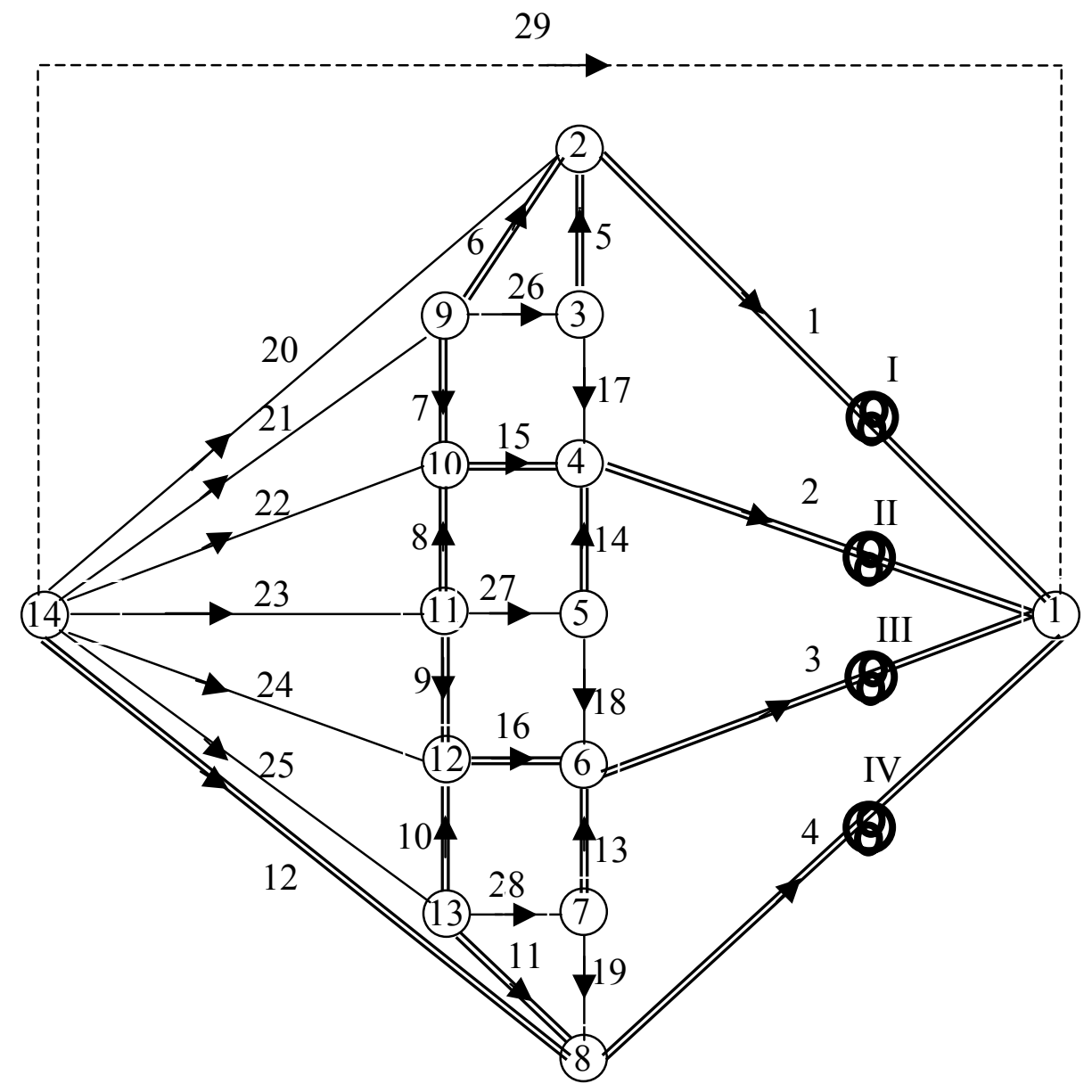

Figure 4.10 -The topology of the 16 meshes, 14 nodes and 29 branches network. The resistance factors in N.s $/ \mathrm{m}$ are shown in the table below.

\begin{tabular}{|c|c||c|c|}
\hline Branch No. & $\begin{array}{c}\text { Resistance } \\
\text { N.s }{ }^{2} \mathrm{~m}^{8}\end{array}$ & Branch No. & $\begin{array}{c}\text { Resistance } \\
\mathrm{N.s}^{2} / \mathrm{m}^{8}\end{array}$ \\
\hline 1 & 0.7 & 16 & 0.5 \\
\hline 2 & 0.7 & 17 & 0.4 \\
\hline 3 & 0.7 & 18 & 0.4 \\
\hline 4 & 0.7 & 19 & 0.4 \\
\hline 5 & 0.5 & 20 & 0.3 \\
\hline 6 & 0.5 & 21 & 0.3 \\
\hline 7 & 0.5 & 22 & 0.3 \\
\hline 8 & 0.5 & 23 & 0.3 \\
\hline 9 & 0.5 & 24 & 0.3 \\
\hline 10 & 0.5 & 25 & 0.3 \\
\hline 11 & 0.5 & 26 & 0.5 \\
\hline 12 & 0.3 & 27 & 0.5 \\
\hline 13 & 0.5 & 28 & 0.5 \\
\hline 14 & 0.5 & 29 & 0.0 \\
\hline 15 & 0.5 & & \\
\hline
\end{tabular}




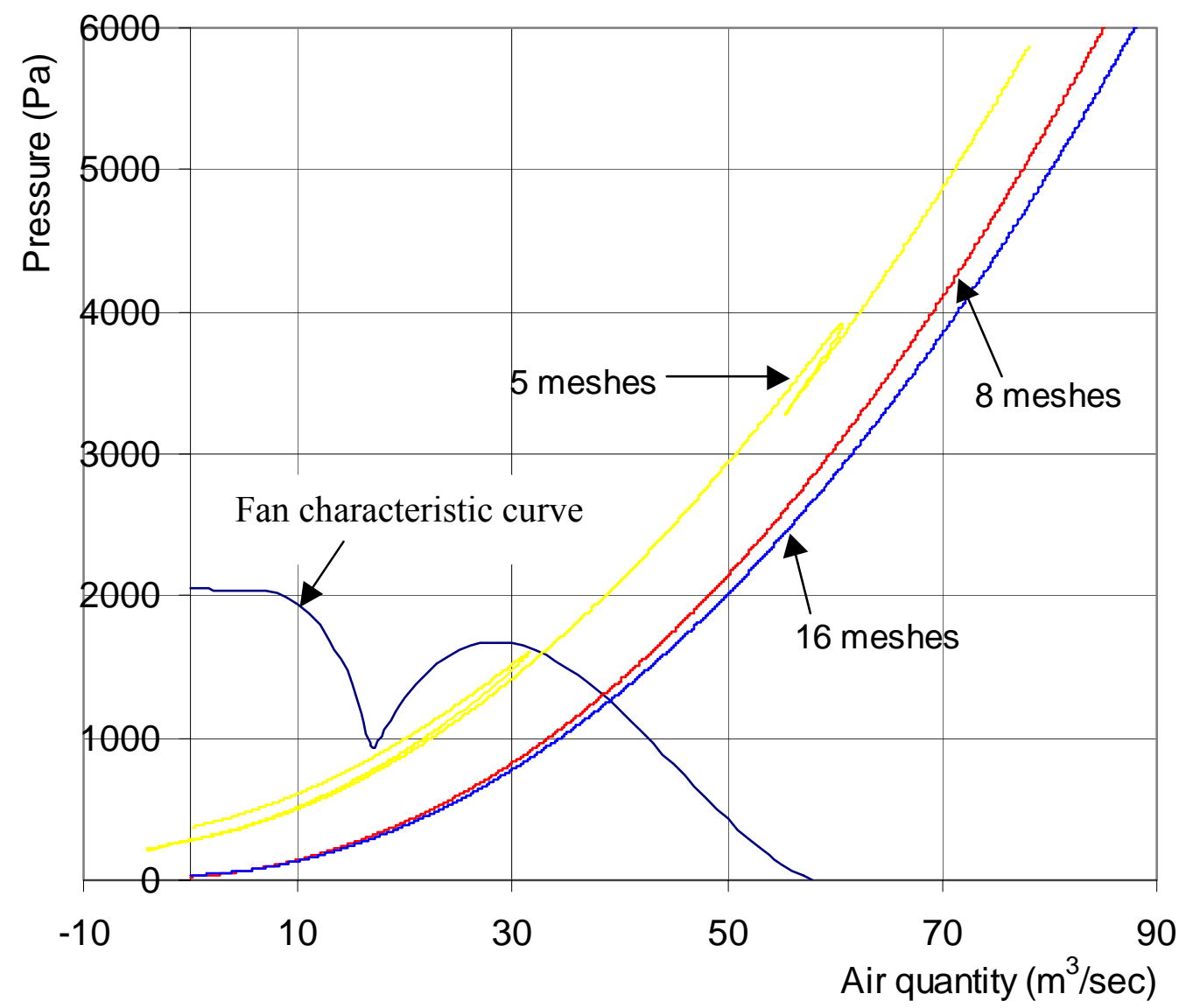

Figure 4.11 -Effect of changing the topology of the network on the shape of subsystem No. 1 characteristic curve. 


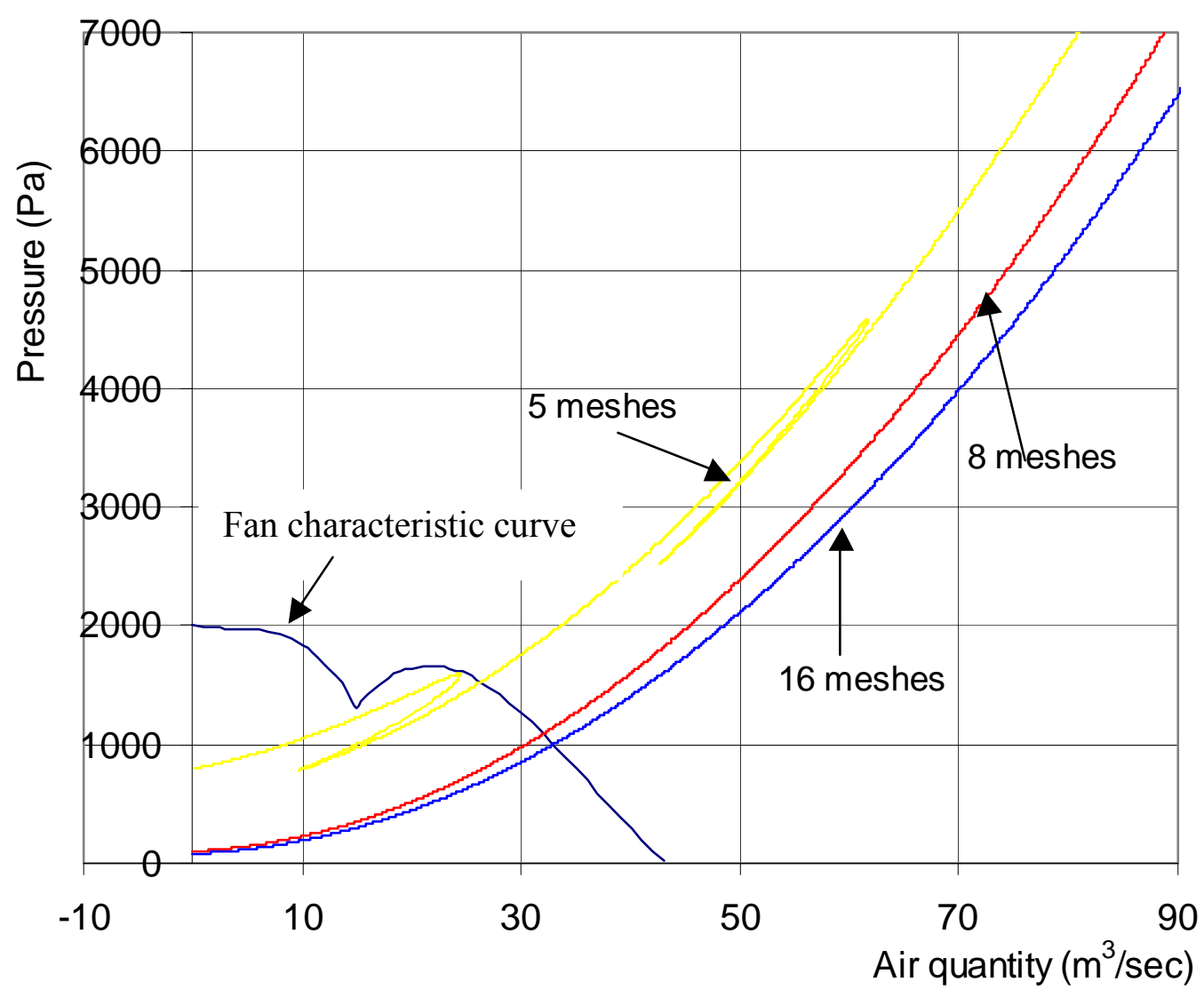

Figure 4.12 -Effect of changing the topology of the network on the shape of subsystem No. 2 characteristic curve. 


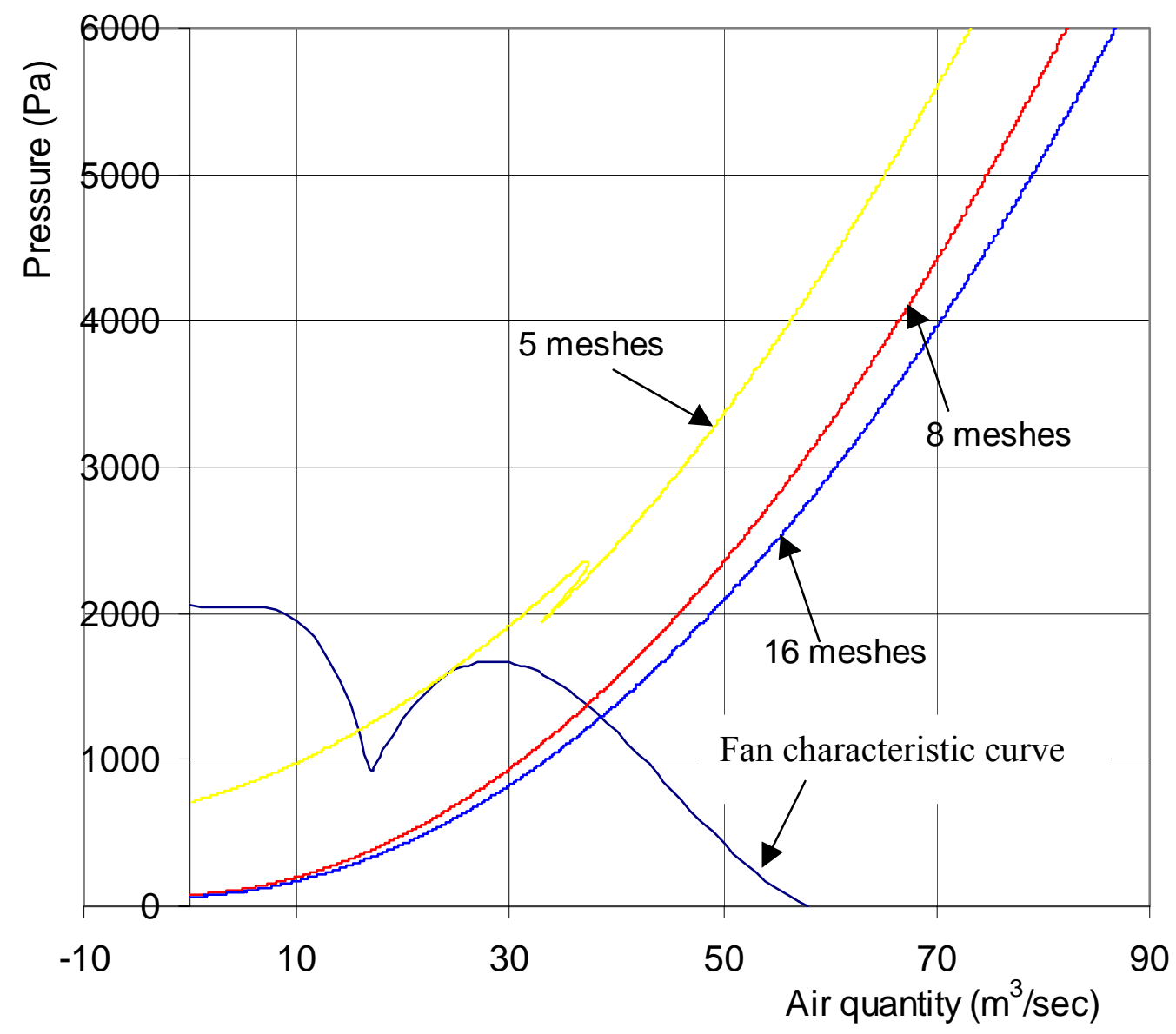

Figure 4.13 -Effect of changing the topology of the network on the shape of subsystem No. 3 characteristic curve. 


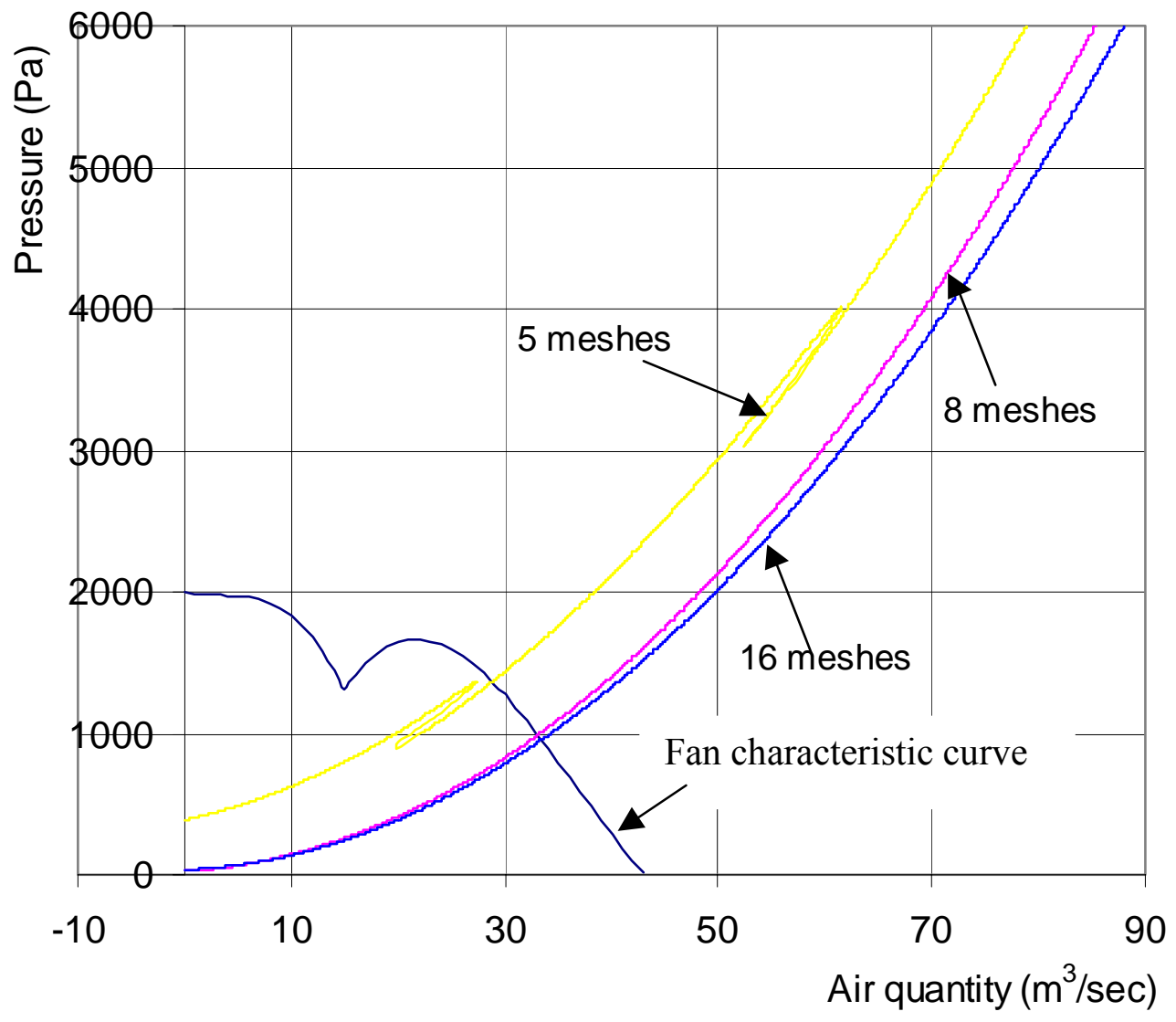

Figure 4.14-Effect of changing the topology of the network on the shape of subsystem No. 4 characteristic curve. 


\subsection{Effect of Number of Fans in the System on the Shape of the Subsystems}

A Multiple fan system is necessary to insure adequate and efficient ventilation in large coal mines throughout the industry. If a fan fails, the hazards associated with interrelated ventilating currents are catastrophical. Kingery and Kapsch (1959) studied three different multiple fan systems in three different coal mines. They agreed that if a mine fan has stopped, one of the following conditions can occur:

- Airflow may reduce in certain sections.

- Air may short circulate and reverse in returns from other mine sections.

- There may be flow from return airways to intake airways under specific conditions.

To study the effect of changing the number of fans on the shape of the subsystem, the number of fans has been changed from 4 to 3,2 and then 1 in the network shown in Figure 4.15. This network consists of 9 branches and 4 nodes as shown in Figures 4.15A. For convenience in modeling, all inlets of the downcast shafts are depicted as if they are connected to a single inlet node. Similarly, all outlets of the up cast shafts are considered connected to a common outlet node. A hypothetical branch, with zero resistance and two nodes, is added to this network where a return dummy branch, branch No. 10, Figure 4.15 $\mathrm{B}$, connects the intake and the main returns airways. The type of branches is mainly dependant upon the location and the number of fans in the system. A single fan has been installed in branch No. 1, as shown in Figure 4.16-A, which represents a simple single fan system. Five intake branches and one return airway branch is indicated in the network Figure 4.16 -B. This network has been solved by applying the principle of a series- 
parallel connection. The overall equivalent resistance factor of this network equals to $0.8068 \mathrm{~N} . \mathrm{s}^{2} / \mathrm{m}^{8}$

A second axial flow fan has been installed in branch No. 4, Figure 4.17. The airflow direction has been changed in branches 6 and 7 as well as branch No. 4, which was an intake branch in the single fan system. Figures 4.18 and 4.19 show the 3 and 4 fan systems respectively.

Figure 4.20 shows the effect of changing the number of fans from a one-fan system to a four-fan system on the subsystem No. 1. One, two and three fan systems do not have a zigzag part as the subsystem characteristic curves is a strictly increasing function. The subsystem No. 1 characteristic curve, in a two-fan system, lies over the single fan system. It does not pass through the origin but it is very close to it, while it is very clear it does not pass through the origin in three and four-fan systems. Only one operating point has been recognized in all of the systems. The same can be written about the subsystem No. 4 characteristic curve shown in Figure 4.21. Figure 4.22 shows the effect of changing the number of fans in the system, from 3 to 4, on the shape of subsystem No. 3 characteristic curves. Both subsystem characteristic curves have a zigzag part, but in case of a 4-fan system, fan number three works in its unstable zone. Figure 4.23 shows the subsystem No. 2 characteristic curve in a 4-fan system. From the above discussion we can deduce that airflow direction in an airway could be reversed if fans are either added to or removed from a system. Increasing the number of axial flow fans in the system, increasing the number and the sharpness of the turns in the subsystem, and consequently 
A

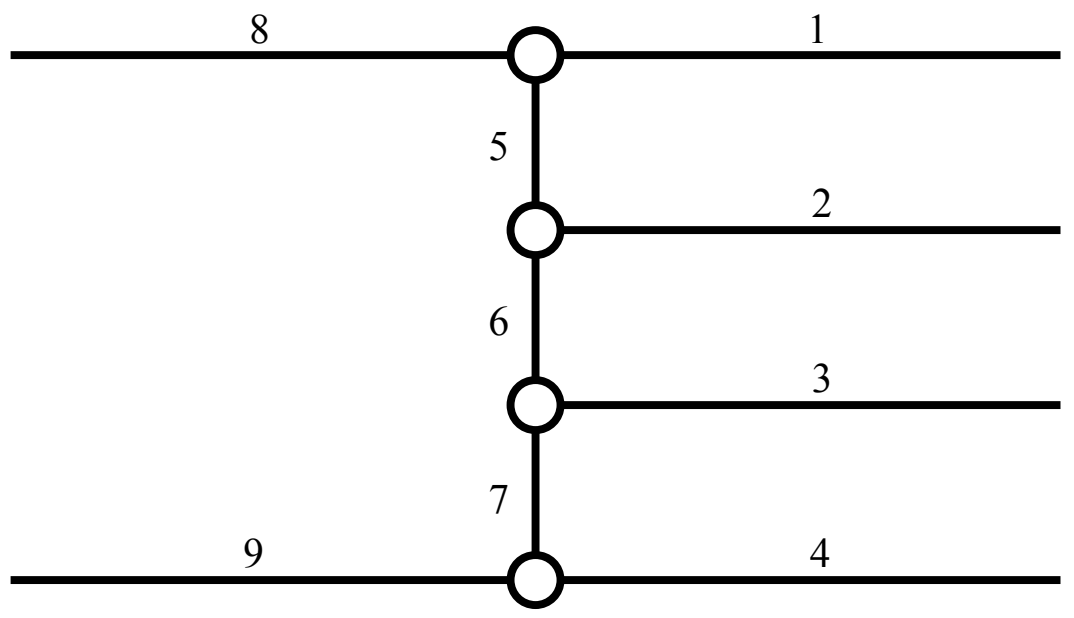

B

10

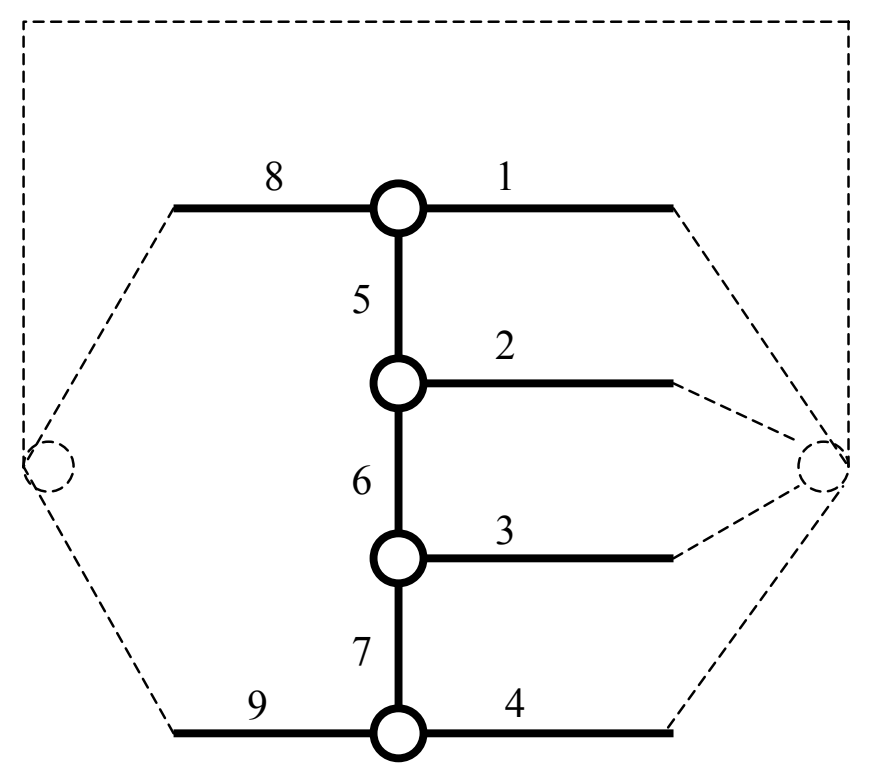

Figure 4.15 -The layout of a network contains 10 branches and 6 nodes.

A. The return dummy branch is not shown.

B. The return dummy branch is shown.

The resistance factors in $\mathrm{N} . \mathrm{s}^{2} / \mathrm{m}^{8}$ in order are $0.7,0.7,0.7,0.7,0.5,0.4$, $0.5,0.3,0.3$, and 0.0 
A

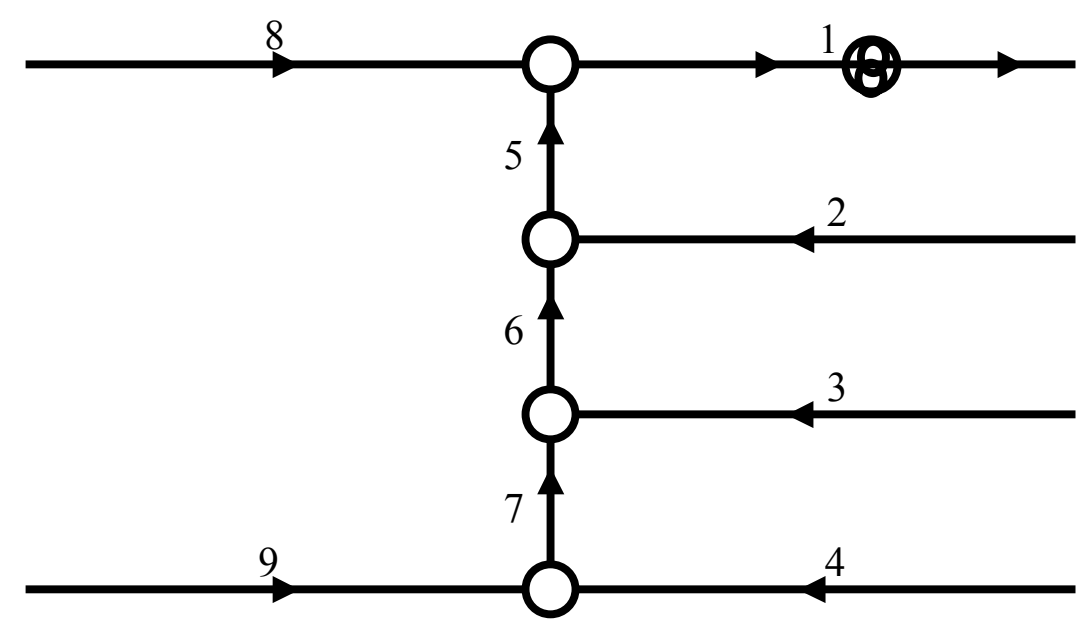

$\mathrm{B}$

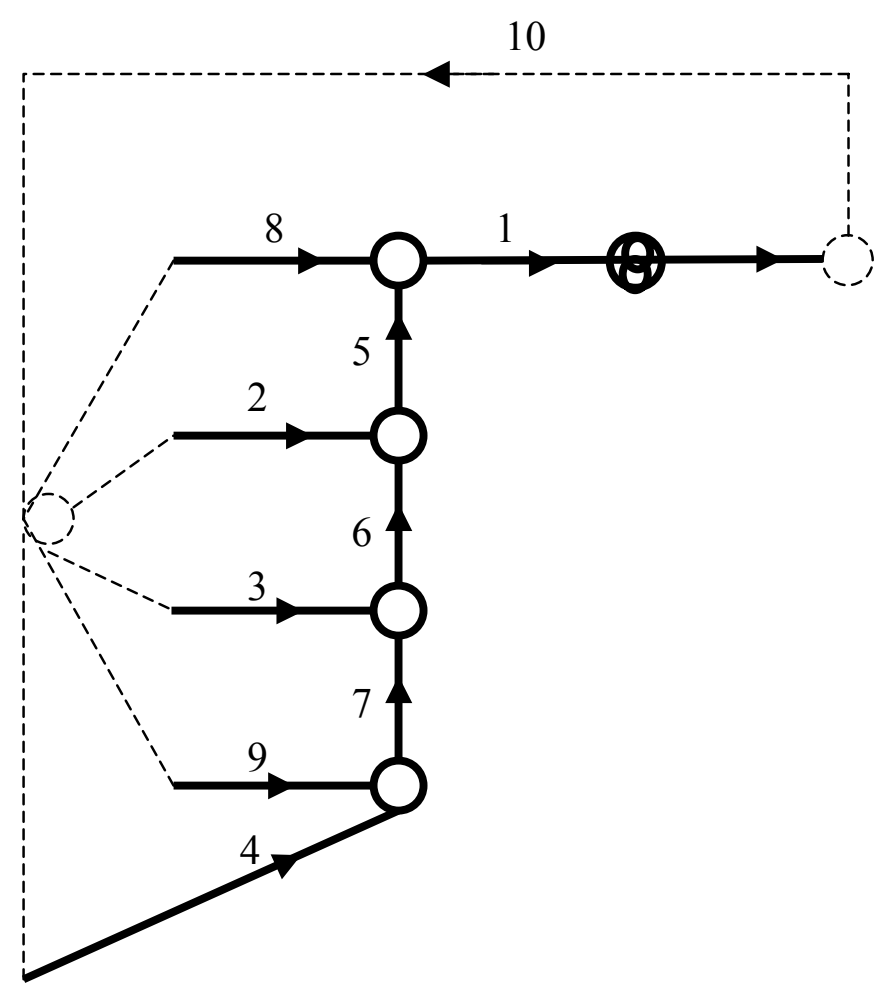

Figure 4.16 -The layout of a network contains 10 branches, 6 nodes and one fan installed in branch No. 1.

A. The return dummy branch is not shown.

B. The return dummy branch is shown. 
A

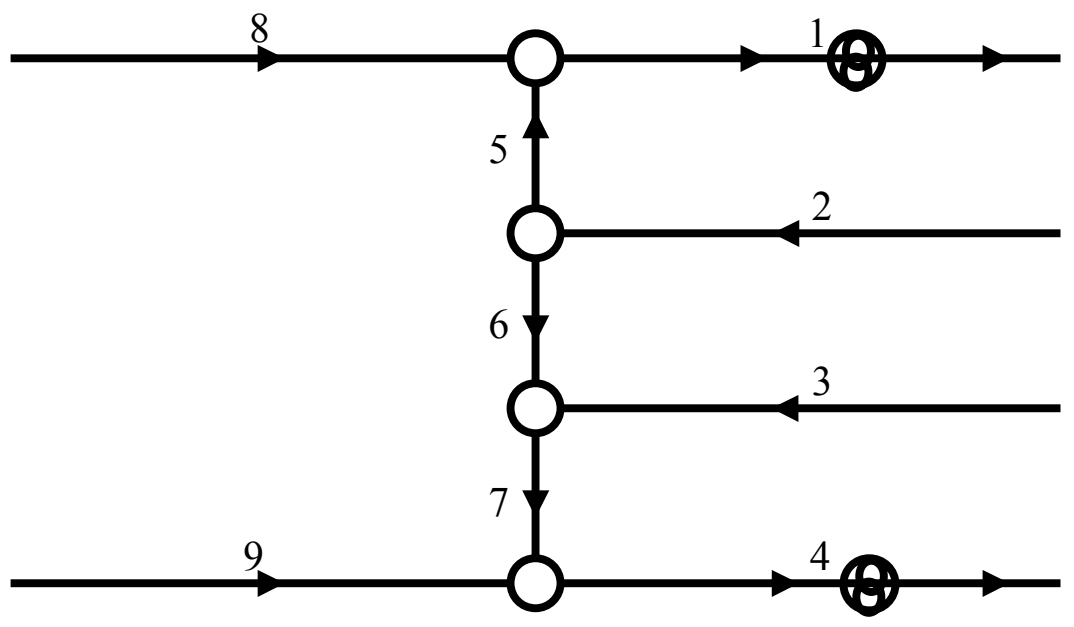

B

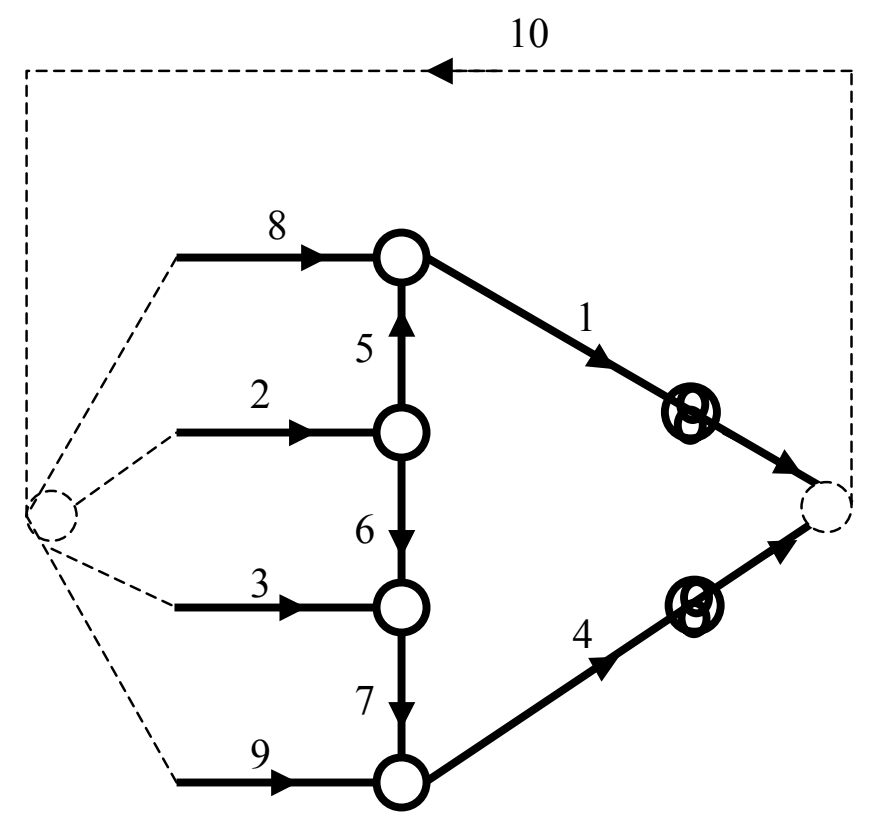

Figure 4.17 -The layout of a network contains 10 branches, 6 nodes and two fans installed in branches Nos. 1 and 4.

A. The return dummy branch is not shown.

B. The return dummy branch is shown. 
A

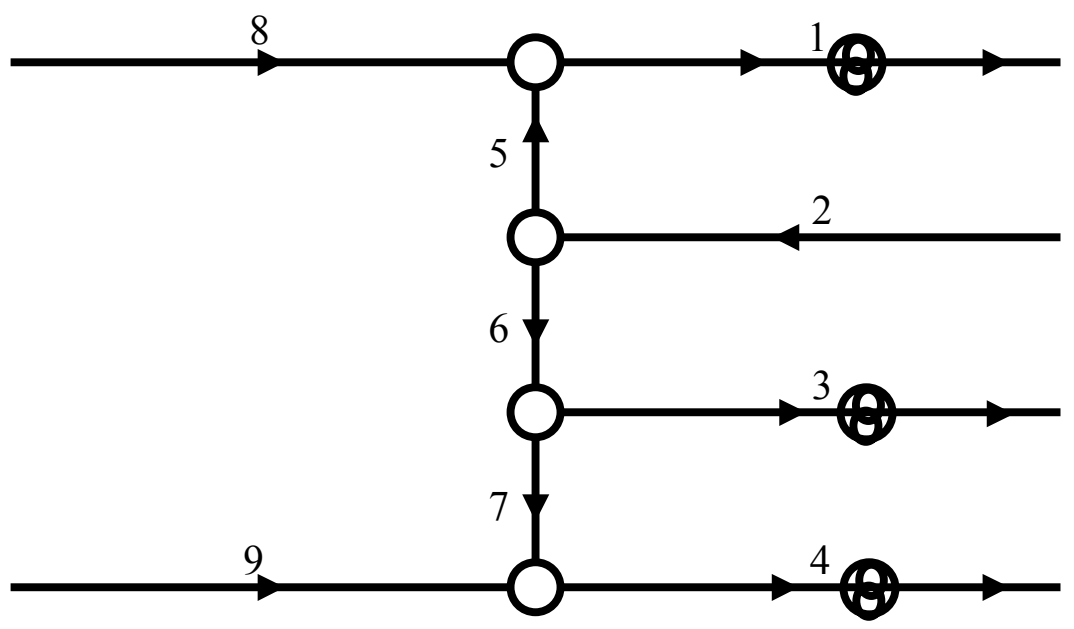

B

10

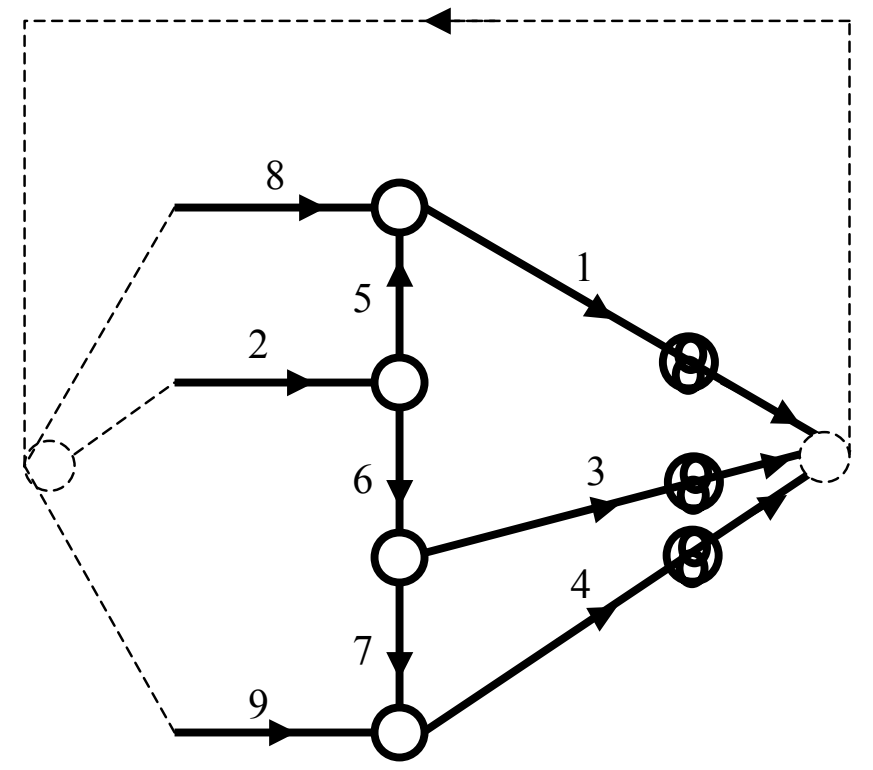

Figure 4.18 -The layout of a network contains 10 branches, 6 nodes and three fans installed in branches Nos. 1, 3, and 4.

A. The return dummy branch is not shown.

B. The return dummy branch is shown. 
A

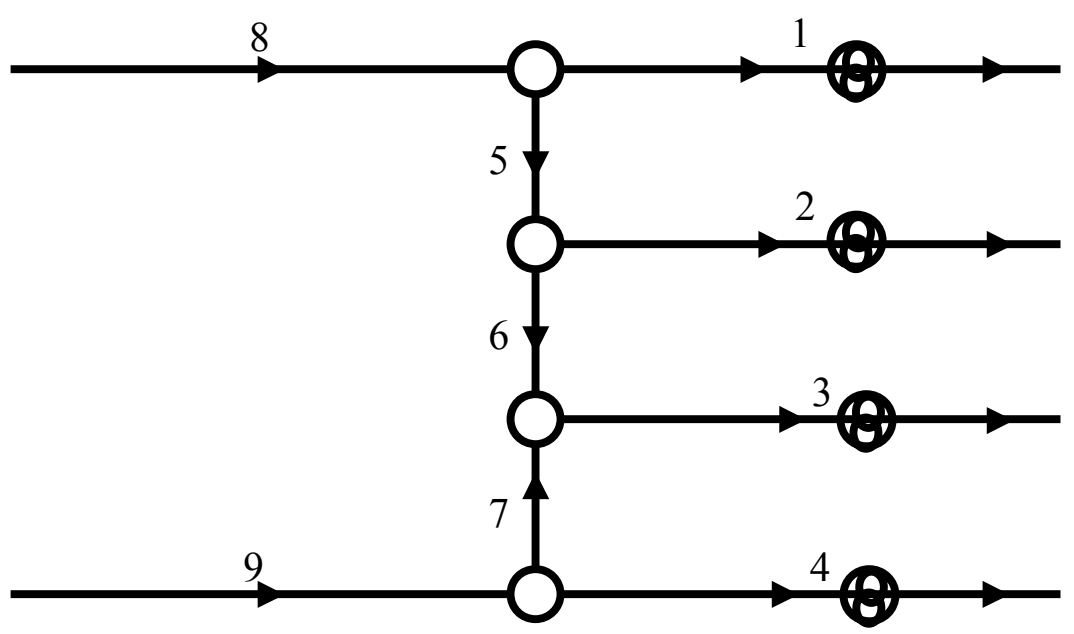

B

10

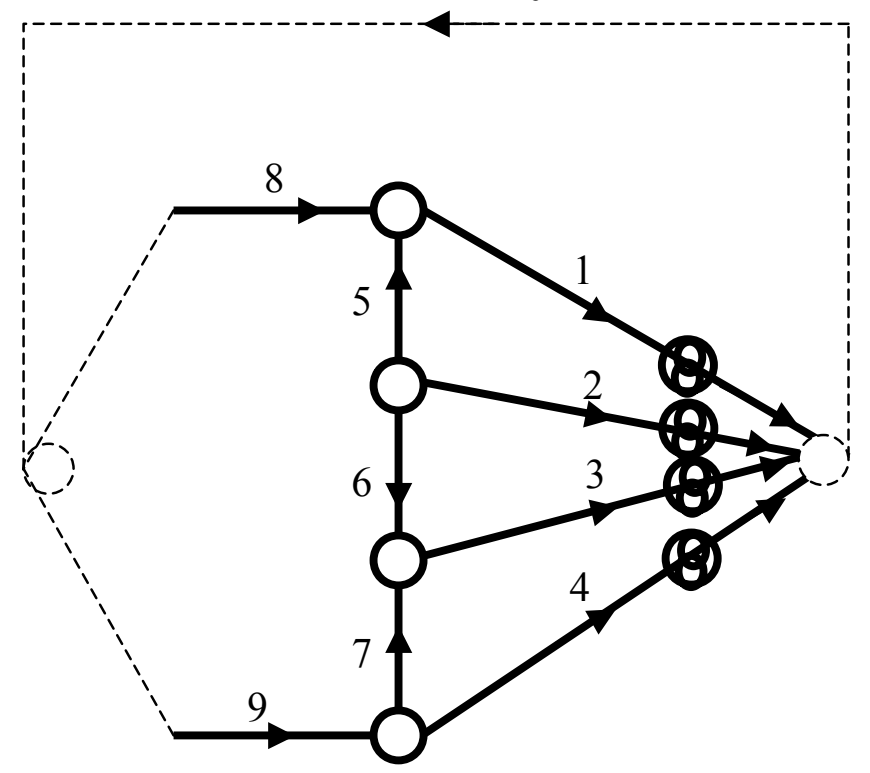

Figure 4.19 -The layout of a network contains 10 branches, 6 nodes and four fans installed in branches Nos. 1, 2, 3, and 4.

A. The return dummy branch is not shown.

B. The return dummy branch is shown. 


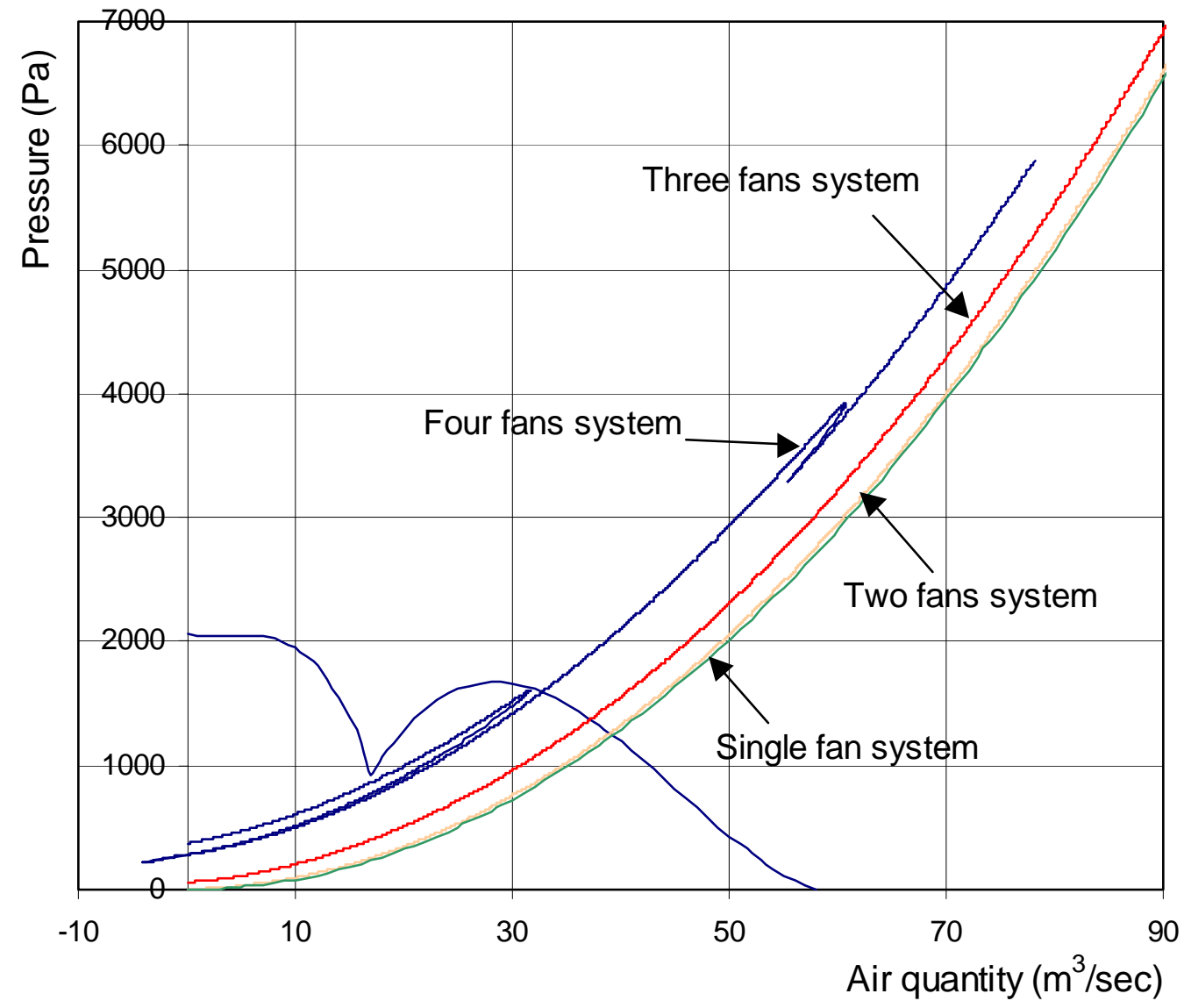

Figure 4.20 -The effect of changing number of fans in the system on the shape of subsystem No. 1 characteristic curve. 


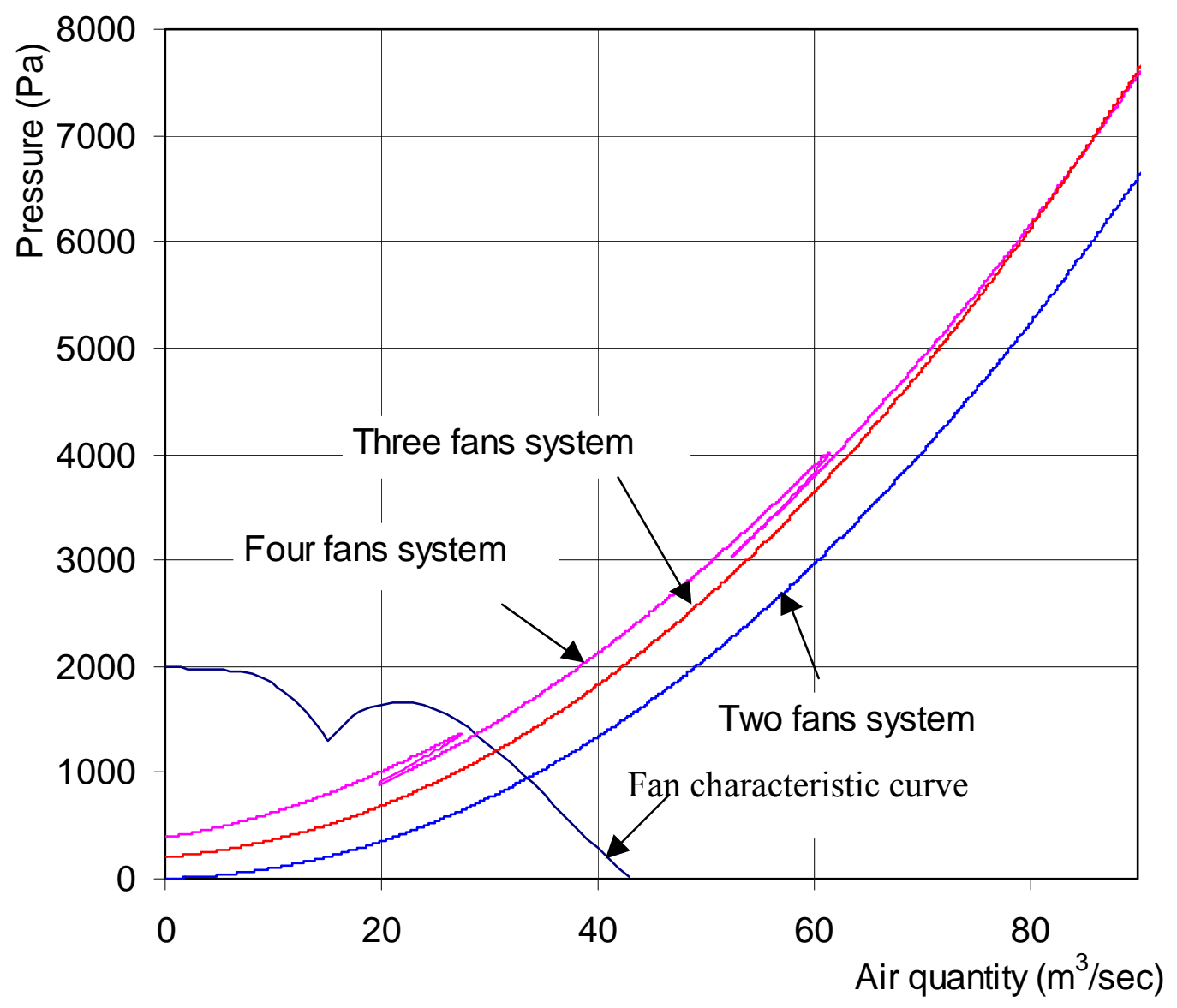

Figure 4.21 -The effect of changing number of fans in the system on the shape of subsystem No. 4 characteristic curve. 


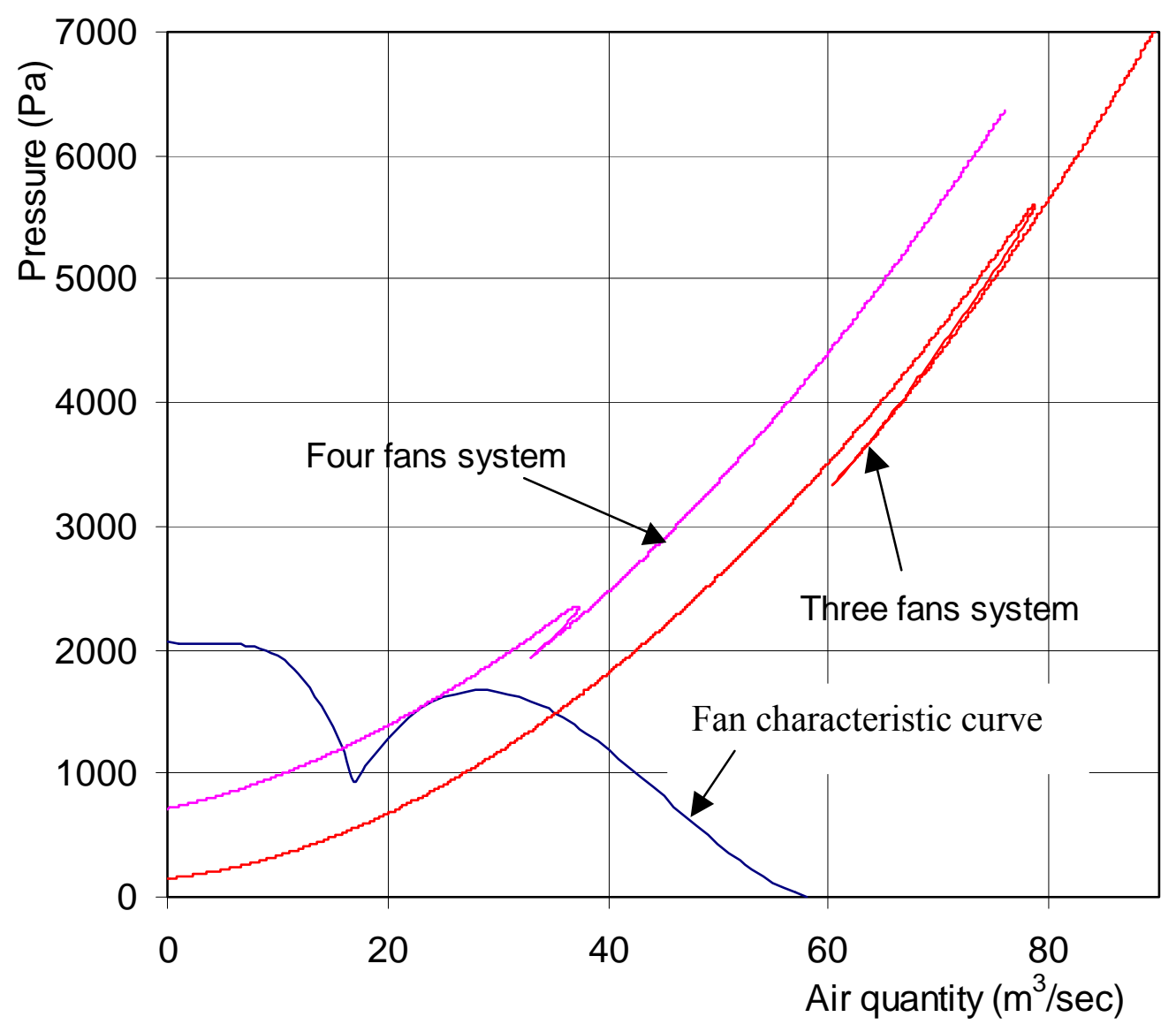

Figure 4.22 -The effect of changing number of fans in the system on the shape of subsystem No. 3 characteristic curve. 


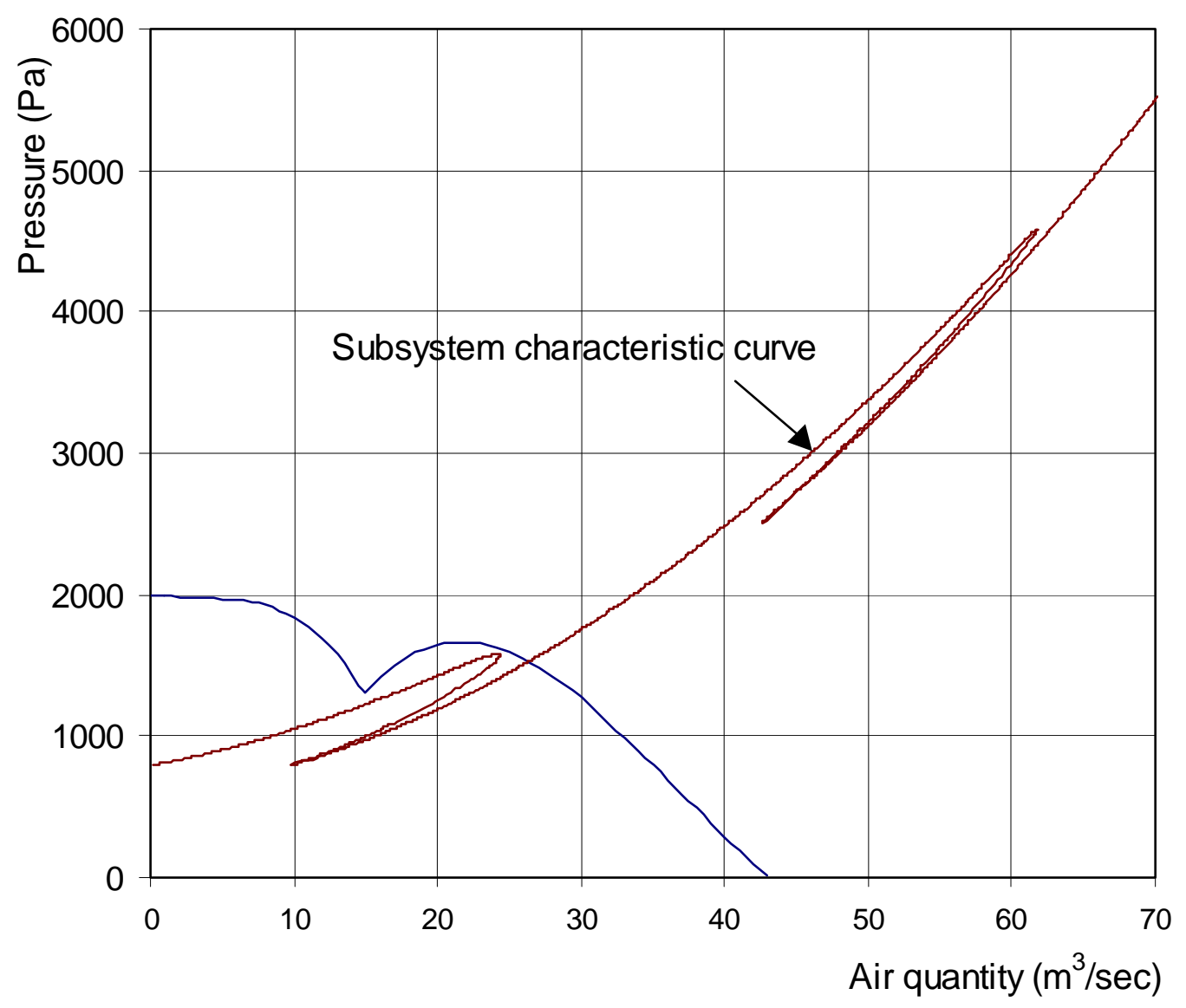

Figure 4.23 -The shape of subsystem No. 2 characteristic curve in a four fans system. 
leads to unstable fan conditions. Decreasing the number of fans in the system not only decreases the number of turns but also reduces the overall resistance of the individual subsystems.

\subsection{Effect of Type of Fans in the System on the Shape of the Subsystems}

As explained before, there are two types of fans used in mine ventilation, axial and centrifugal. Centrifugal fans are more stable and do not have turning points like axial flow fans. If all the fans in the systems were centrifugal, the subsystem characteristic curve would be a strictly increasing function, like the single fan system. To study the effect of changing the type of fans in the system on the shape of the subsystem, first a fan in branch No. 3 (Figure 3.4) has been replaced from an axial flow fan type A to a centrifugal flow fan type C (Figure 3.4), then a fan in branch 2, a fan in branch No. 4, and lastly a fan in branch No. 1 is replaced with a type C fan.

Figures 4.24 to 4.28 show the effect of changing the fans in the system gradually from 4 axial flow fans to 4 centrifugal fans on subsystem No. 1 characteristic curve. The effect is not large in the slope or on the value of the y-axis, but it is considerable on the shape of the curve itself. The zigzagging parts of the curve vanish when two or more centrifugal fans are installed in the system. All the curves intersect with the y-axis at the same point. The effect is almost the same on the subsystem No. 2, but it has turning parts until four centrifugal fans have been installed in the system, as shown in Figures 4.29 to 4.33. Figures 4.34 through 4.38 and Figures 4.39 through 4.43 show this effect on subsystem No. 3 and 4 respectively, which is the same trend as subsystem No. 1. The 
number of operating points is not affected by changing the types of fans in the system. All systems studied have only one operating point. The number and the type of fan inside subsystem No. 1, shown in Figure 4.24, is the same as that in Figure 4.25. The difference between these two cases is the fan type installed in branch No. 1. Thus the shape of subsystem No. 1 characteristic curve in both cases is the same. The same phenomena has been recognized in Figures 4.30 and 4.31, Figures 4.34 and 4.35 and Figures 4.41 and 4,42 which represent subsystem 2, 3 and 4 respectively, when replacing the axial flow fan installed in the reference branch by a centrifugal fan. Thus, we can conclude that the shape of the subsystem characteristic curve does not depend on the associated fan, but rather it depends mainly on the fans inside each subsystem.

\subsection{Effect of Fan Speed in the System on the Shape of the Subsystem}

Changing the speed of a fan in a system effect the fan performance of the fan as has been discussed in section 2.3. On other hand, quantity varies almost proportionally and head directly to a fractional exponent with a change in pitch angle of the fan (Morris and Hinsley, 1951). There is no specified relation between the pressure or air quantity delivered from a fan and the blade angle. Fan manufacturers usually provide mine ventilation engineers with graphs or tables that show the relation between pressure and air quantity delivered from a fan at different blade angles. Large mine axial flow fans are usually variable-pitch fans. The angle of inclination of the blades can be changed over a relatively narrow range to safe operating conditions of the fan $\left(10\right.$ to $\left.30^{\circ}\right)$. From the illustrative example in Chapter 3, fan No. three is the only fan that works in its unstable zone (Figure 3.8). To study the effect of changing fan speed on the shape of a subsystem, 


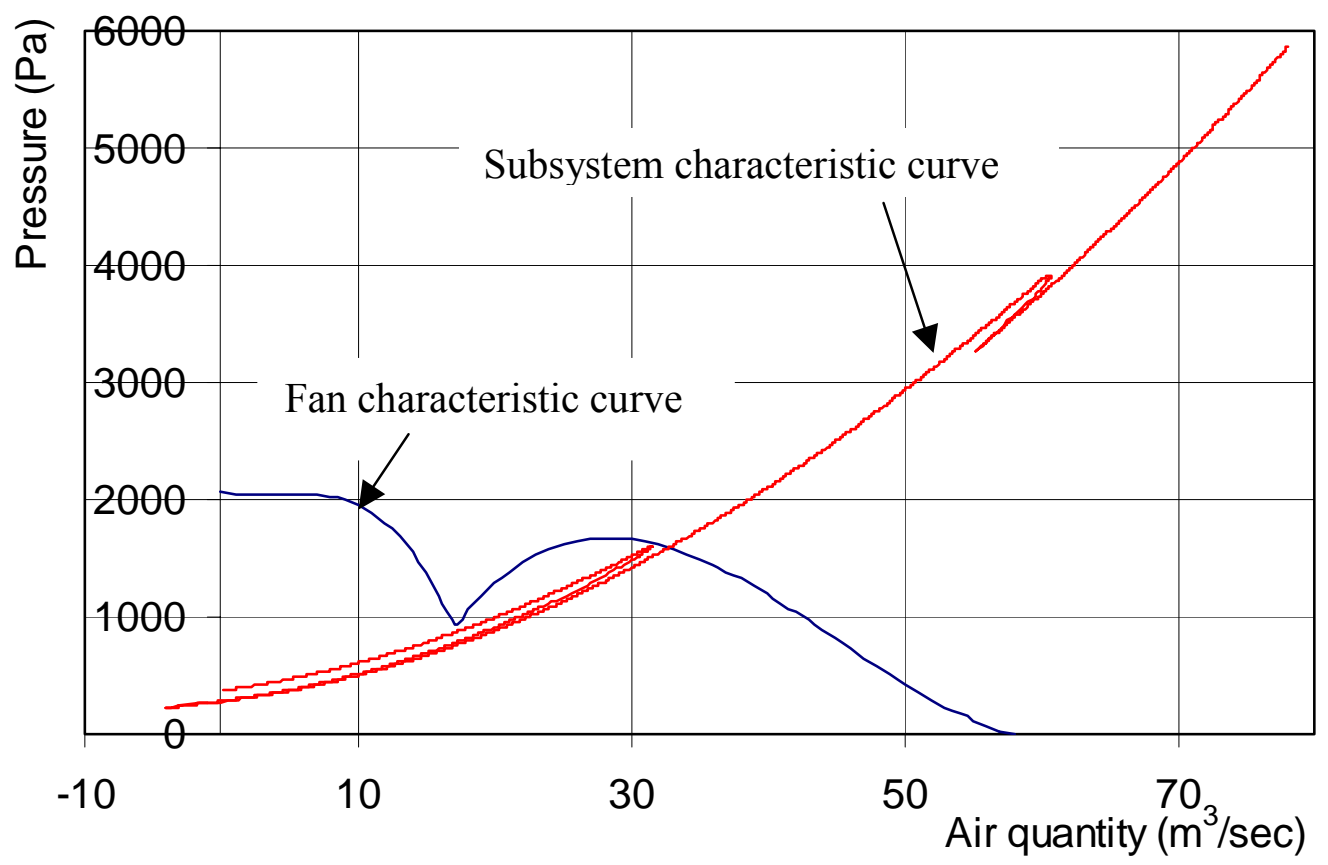

Figure 4.24 -Shape of subsystem No. 1 characteristic curve in a 4 axial flow fans system.

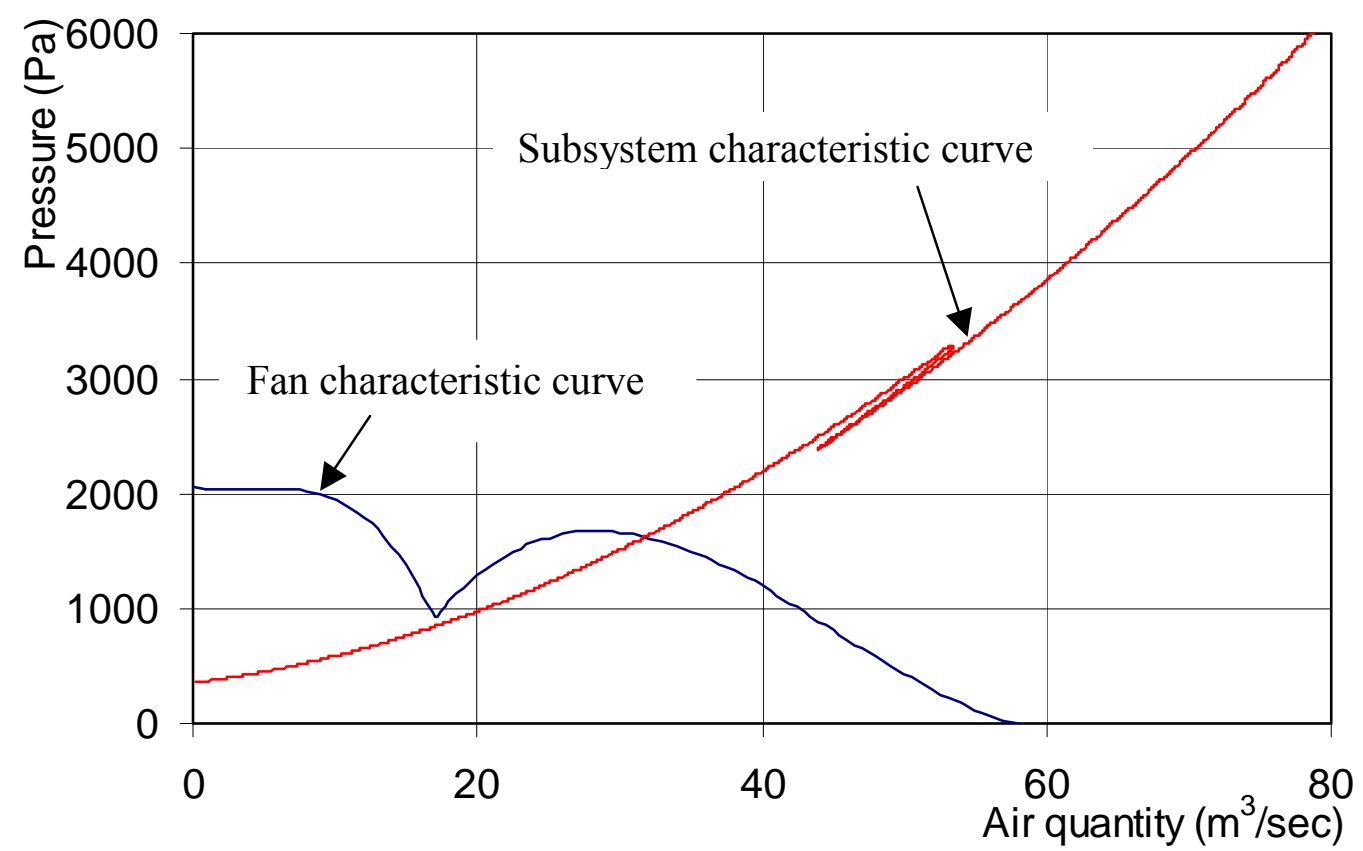

Figure 4.25 -Shape of subsystem No. 1 characteristic curve in a 3 axial and 1 centrifugal flow fans system. 


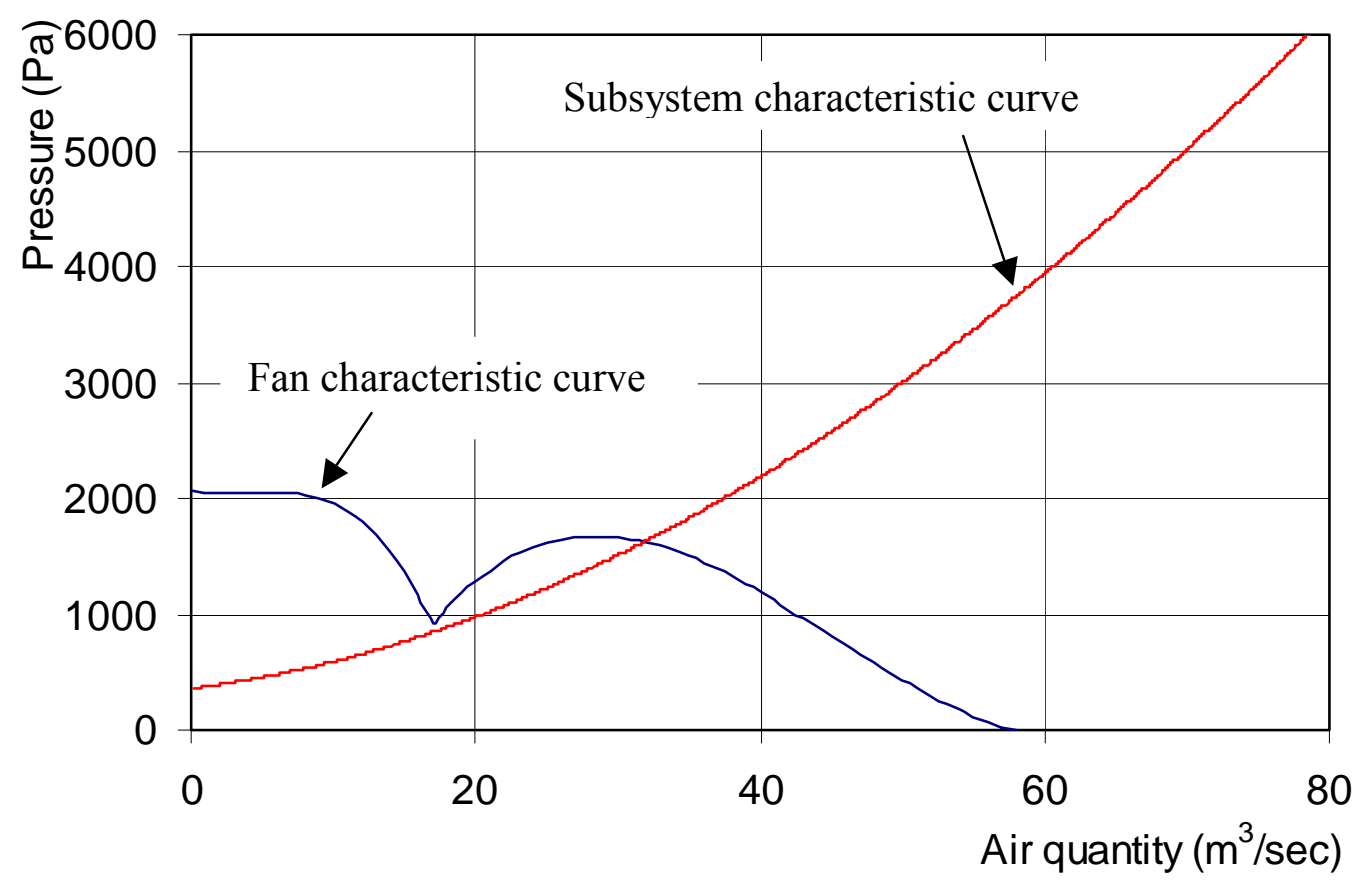

Figure 4.26 -Shape of subsystem No. 1 characteristic curve in a 2 axial and 2 centrifugal flow fans system.

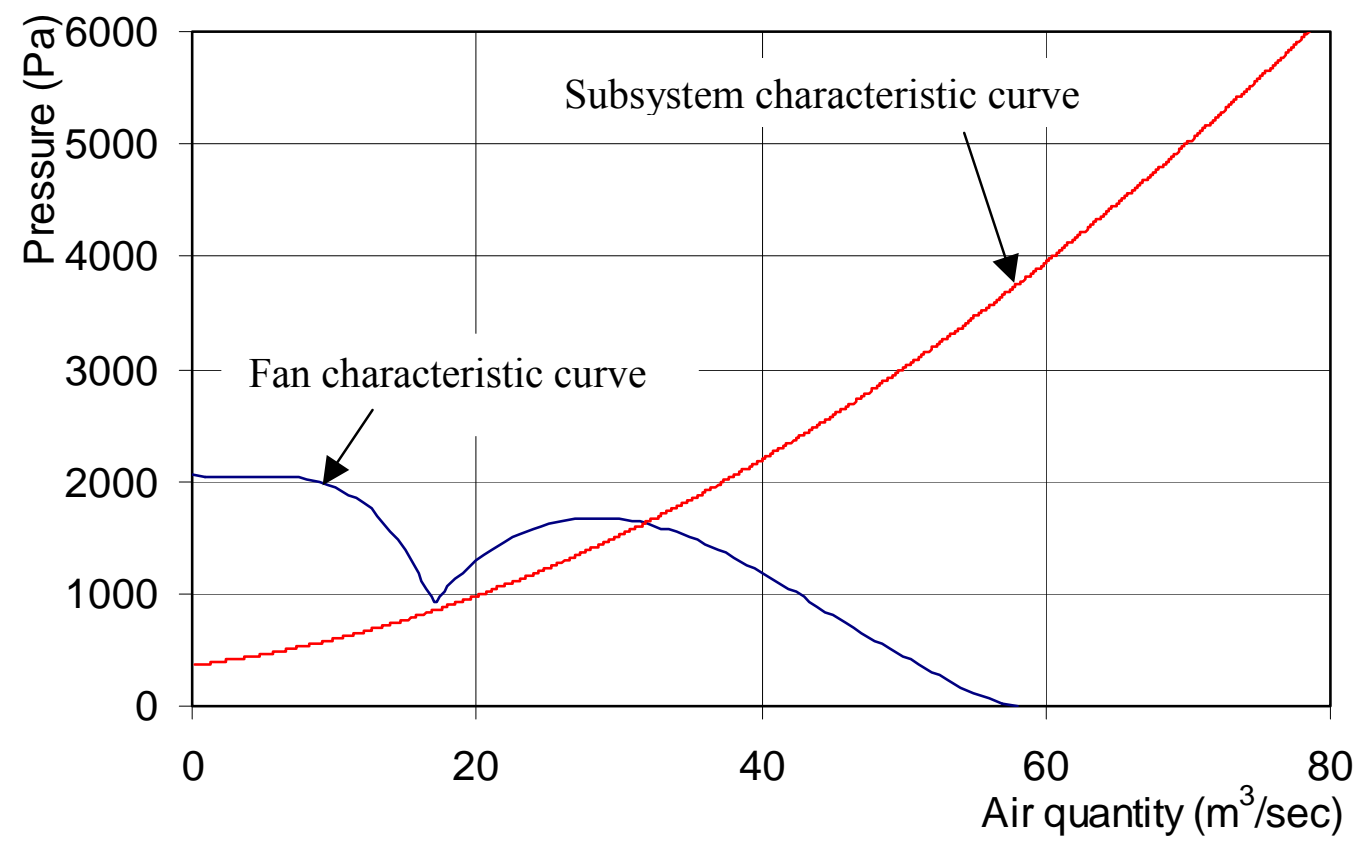

Figure 4.27 -Shape of subsystem No. 1 characteristic curve in a 1 axial and 3 centrifugal flow fans system. 


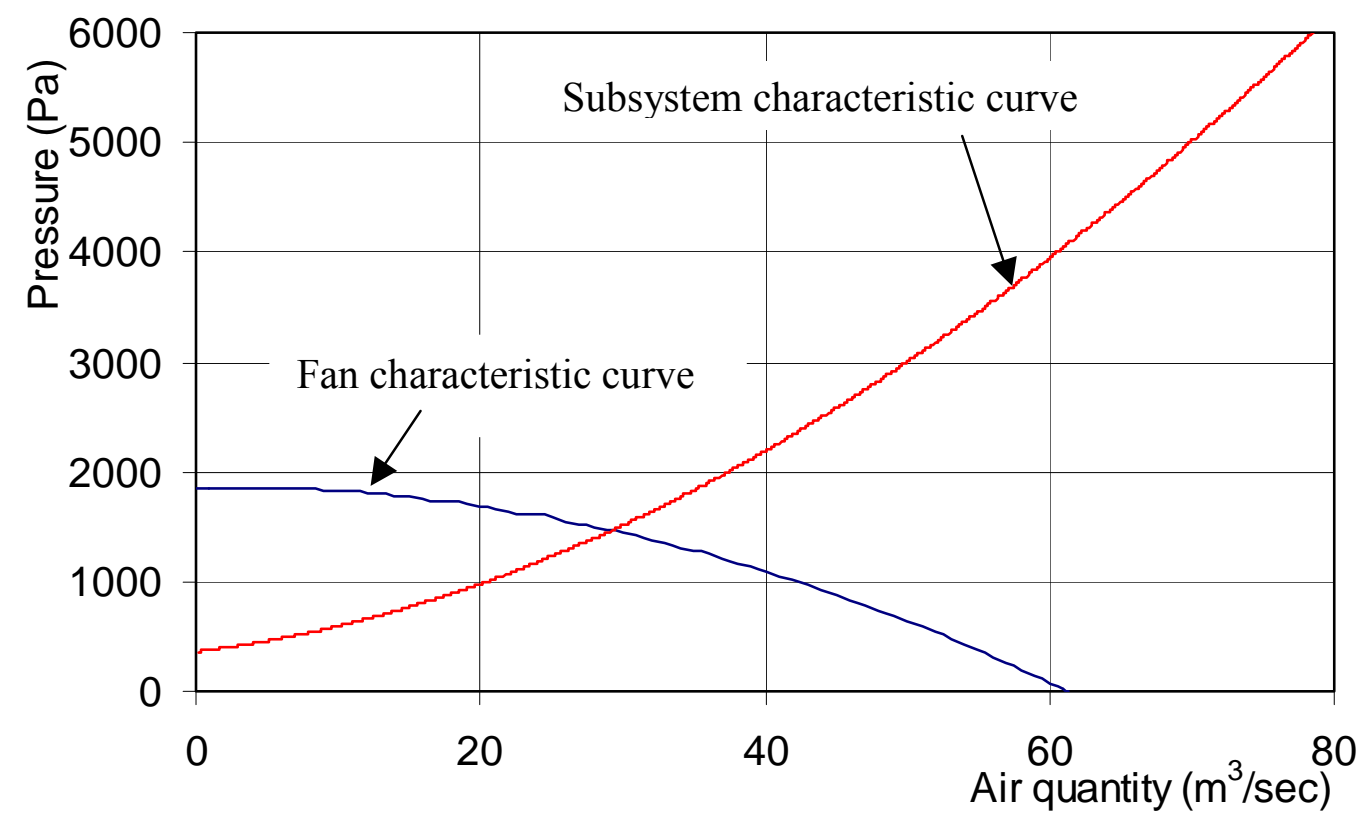

Figure 4.28 -Shape of subsystem No. 1 characteristic curve in a 4 centrifugal flow fans system.

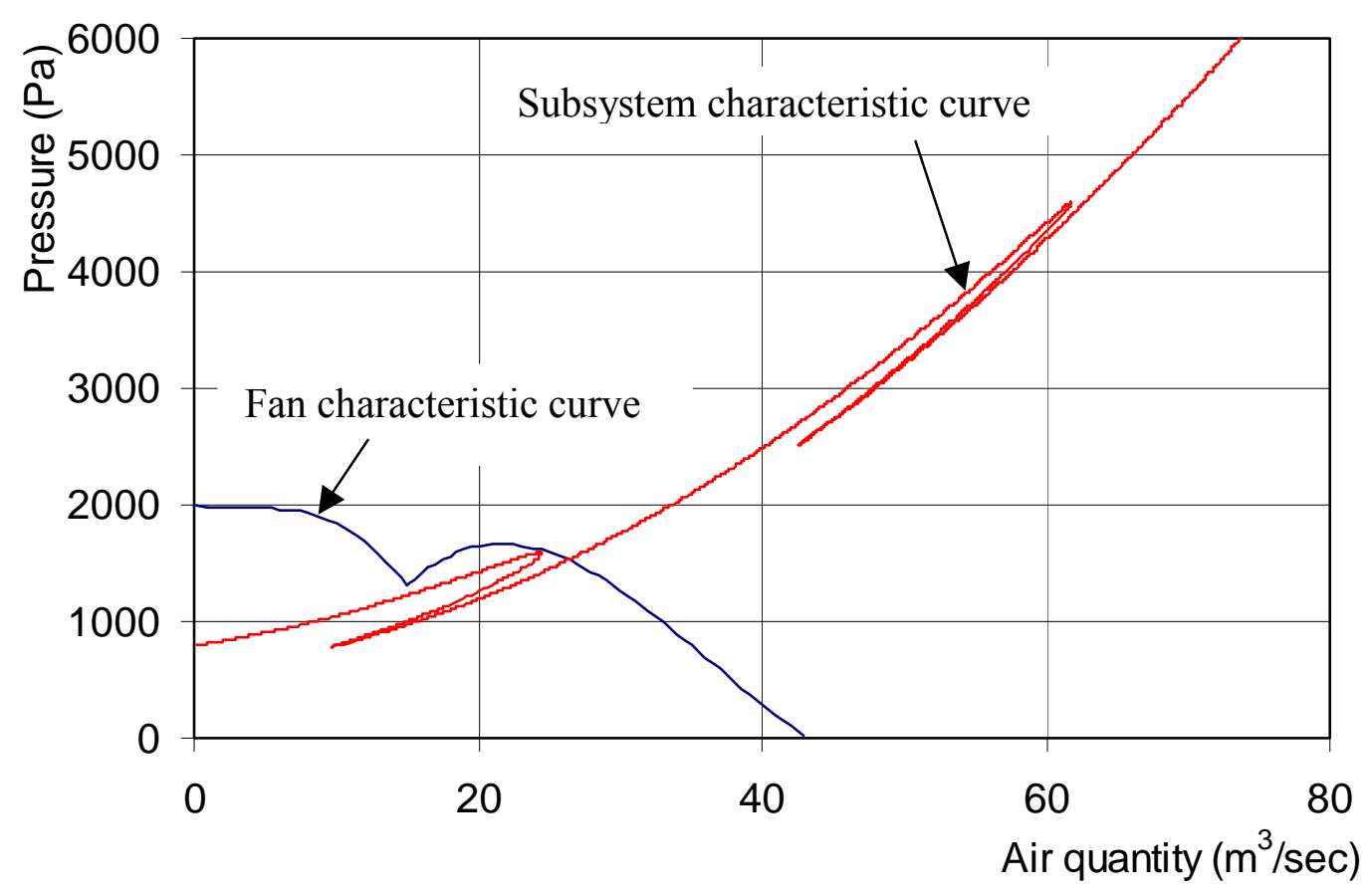

Figure 4.29 -Shape of subsystem No. 2 characteristic curve in a 4 axial flow fans system. 


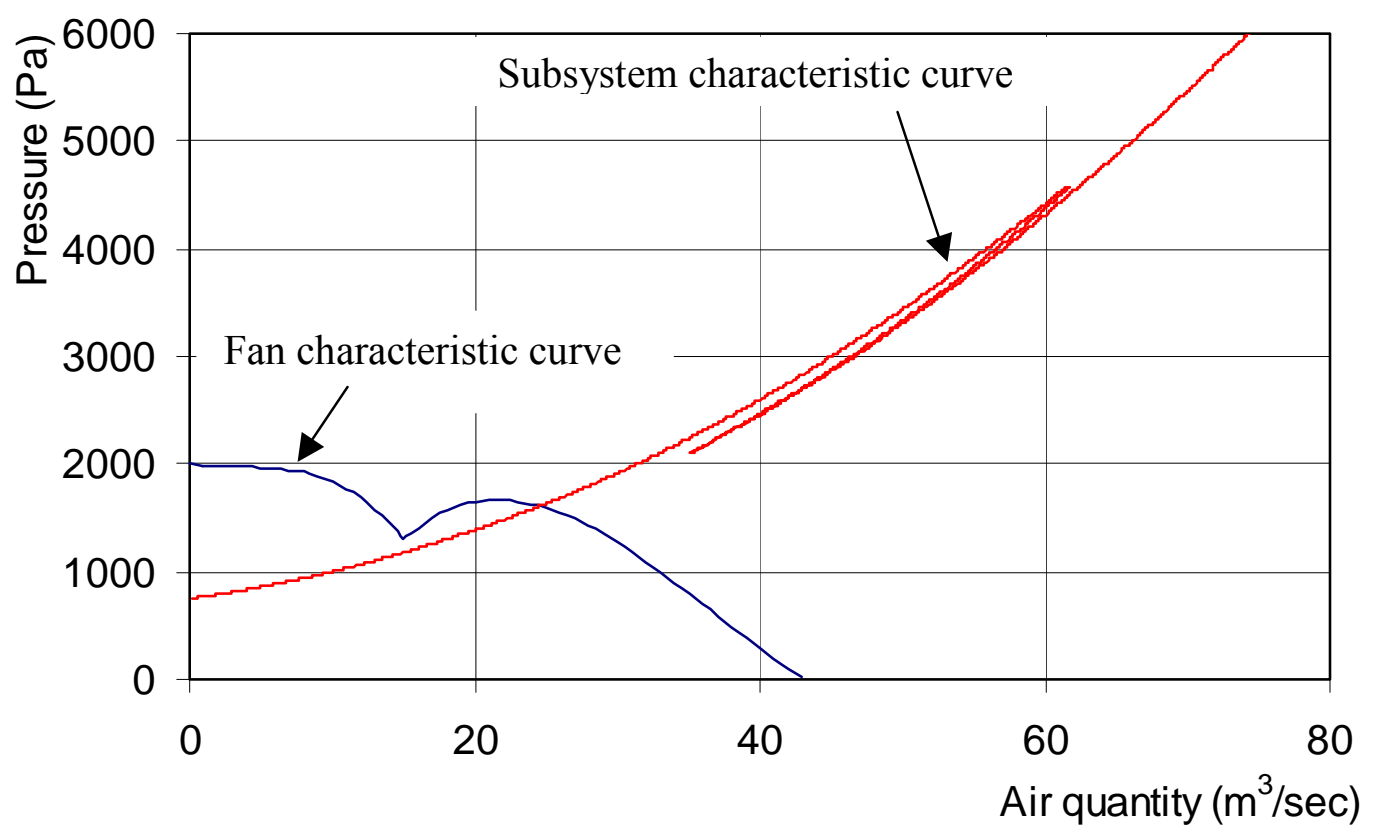

Figure 4.30 -Shape of subsystem No. 2 characteristic curve in a 3 axial and 1 centrifugal flow fans system.

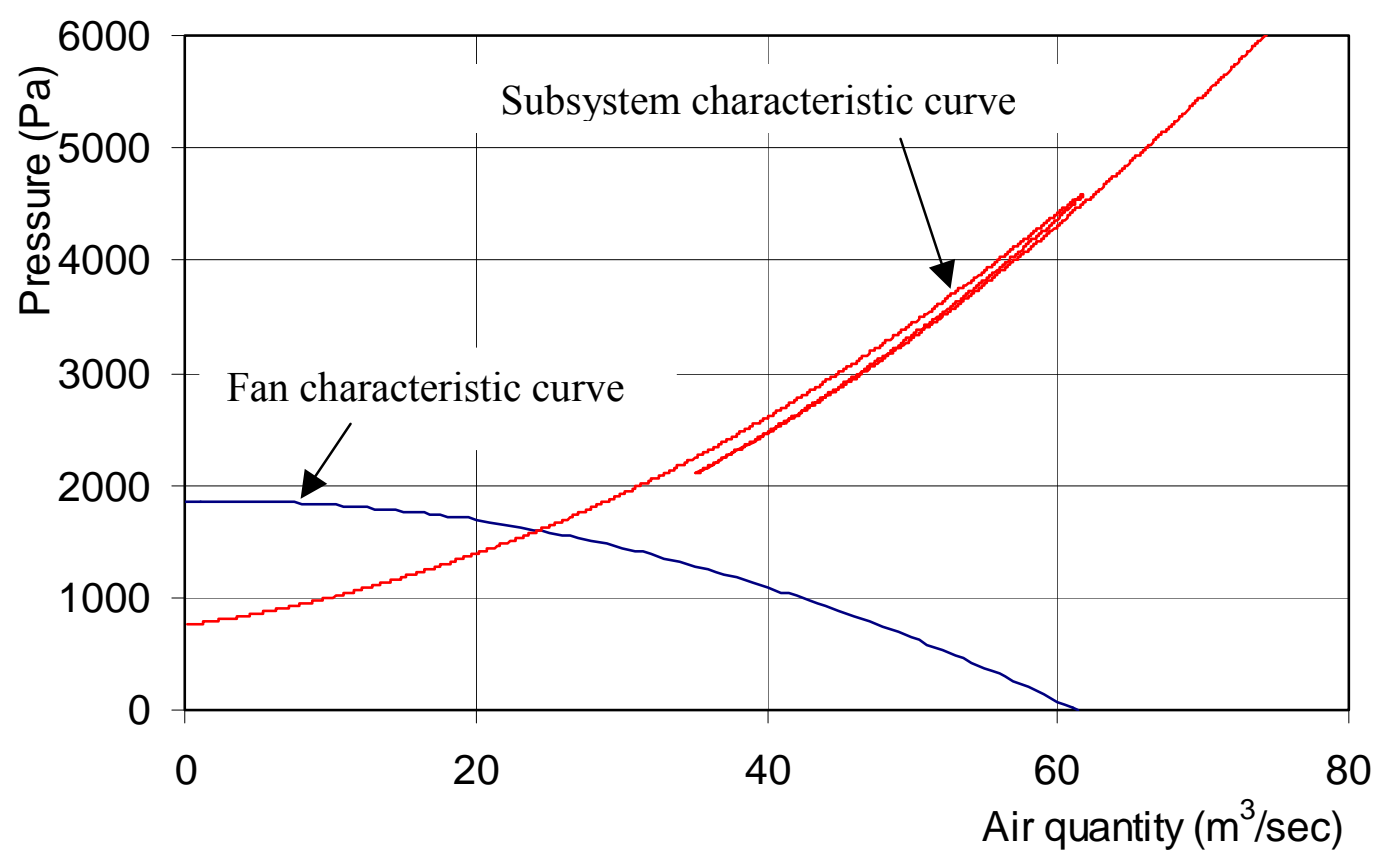

Figure 4.31 -Shape of subsystem No. 2 characteristic curve in a 2 axial and 2 centrifugal flow fans system. 


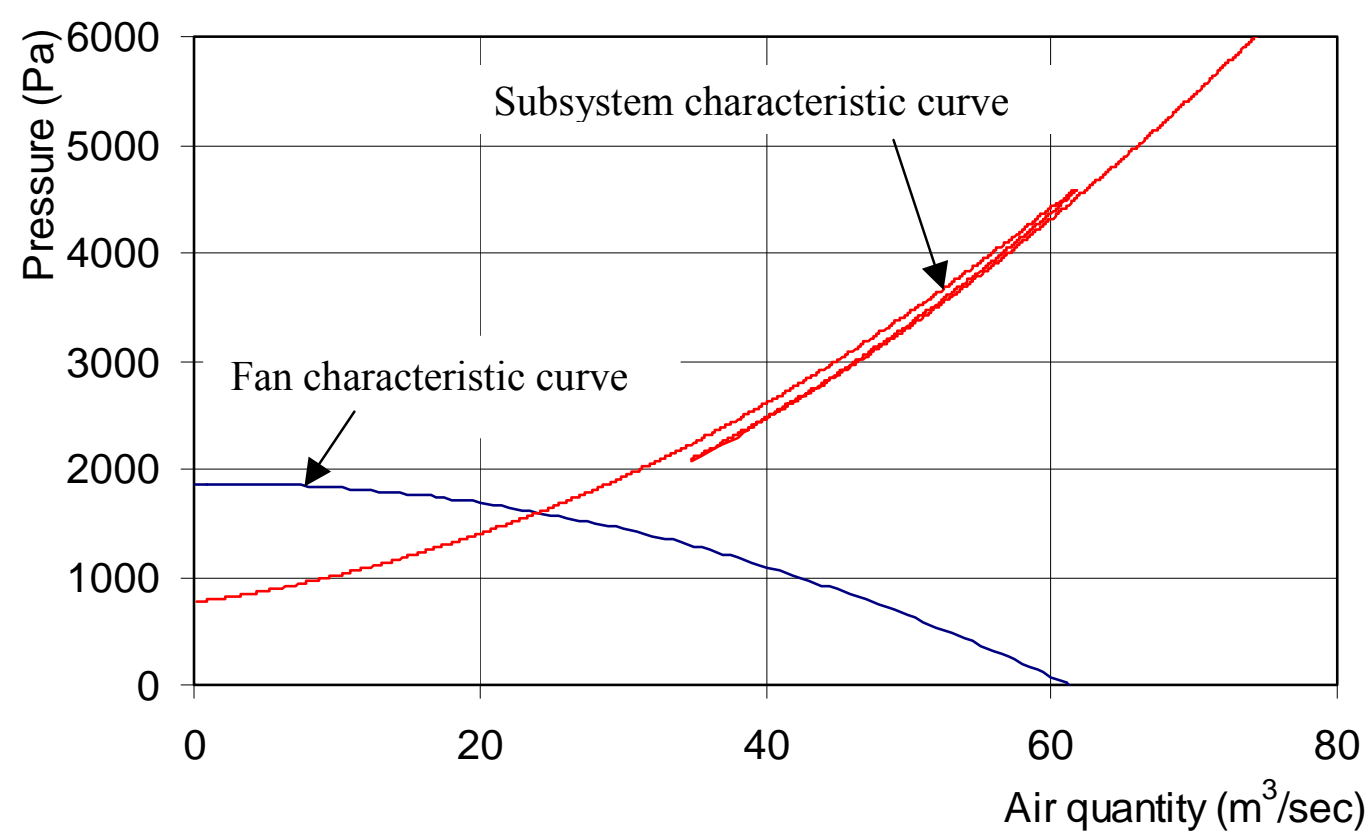

Figure 4.32 -Shape of subsystem No. 2 characteristic curve in a 1 axial and 3 centrifugal flow fans system.

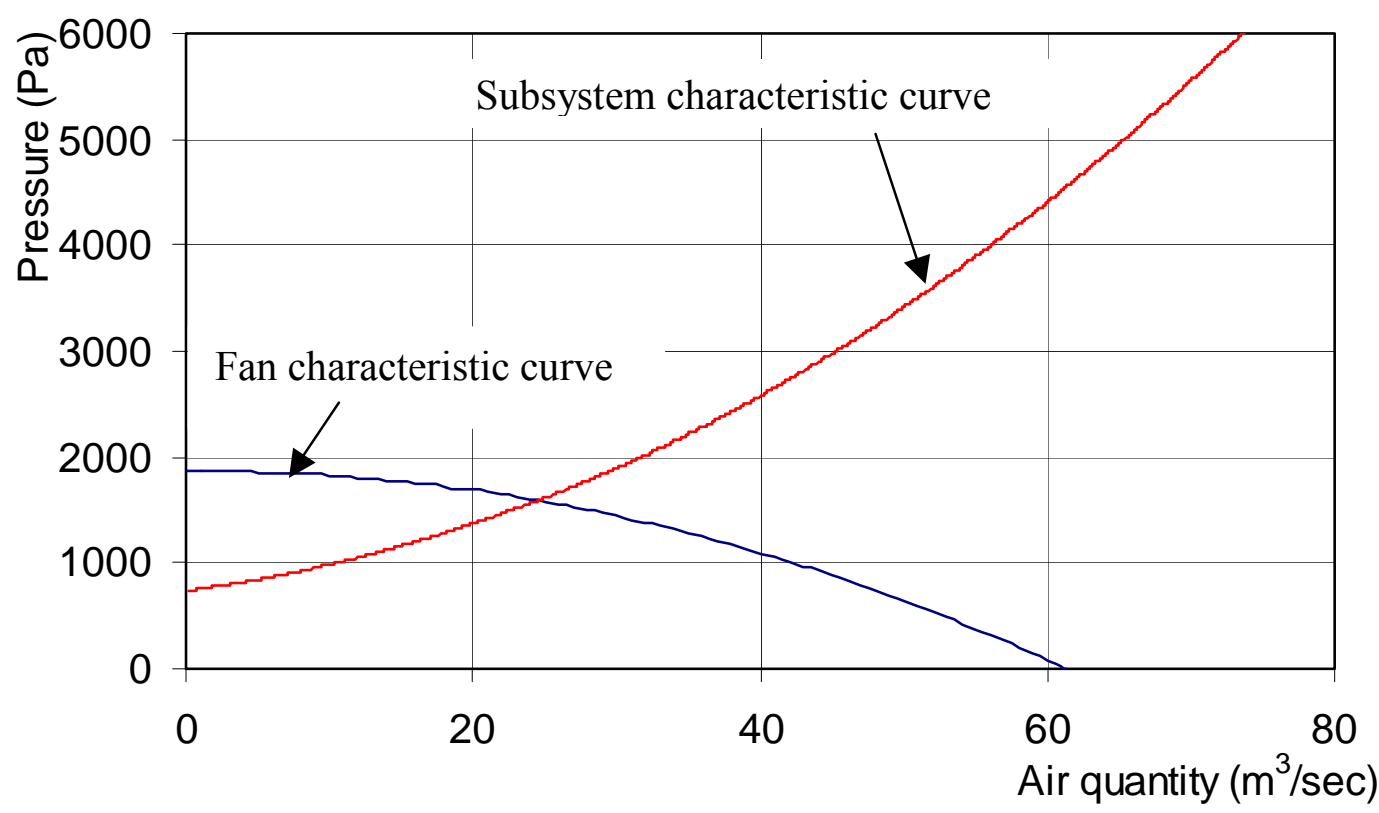

Figure 4.33 -Shape of subsystem No. 2 characteristic curve in a 4 centrifugal flow fans system. 


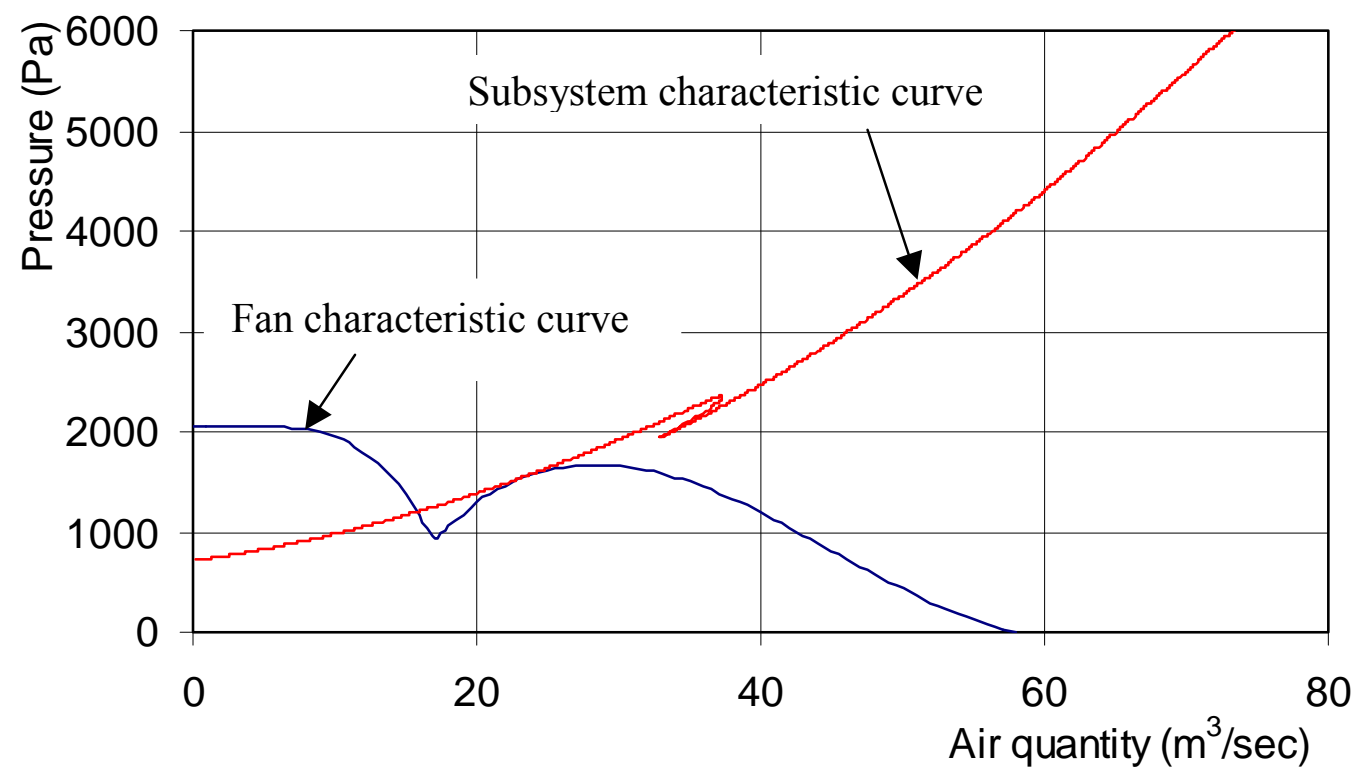

Figure 4.34 -Shape of subsystem No. 3 characteristic curve in a 4 axial flow fans system.

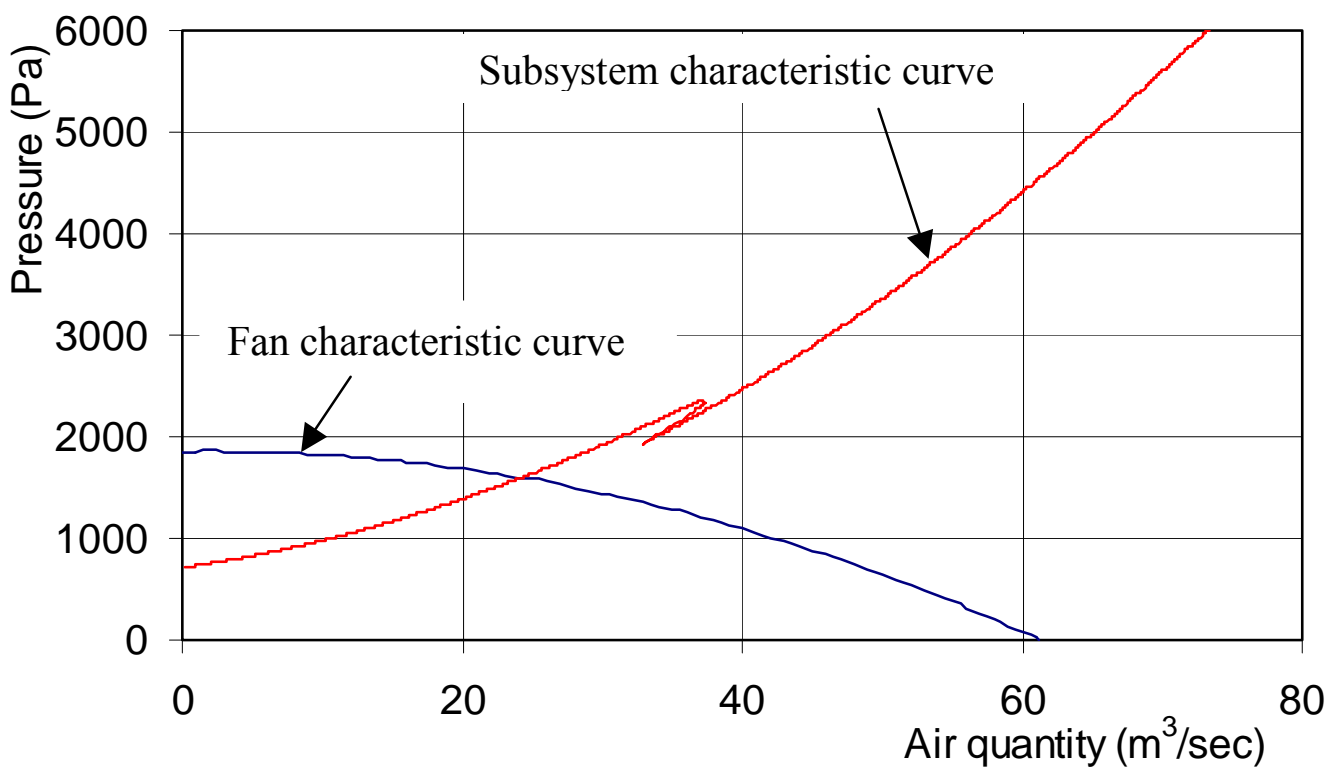

Figure 4.35 -Shape of subsystem No. 3 characteristic curve in a 3 axial and 1 centrifugal flow fans system. 


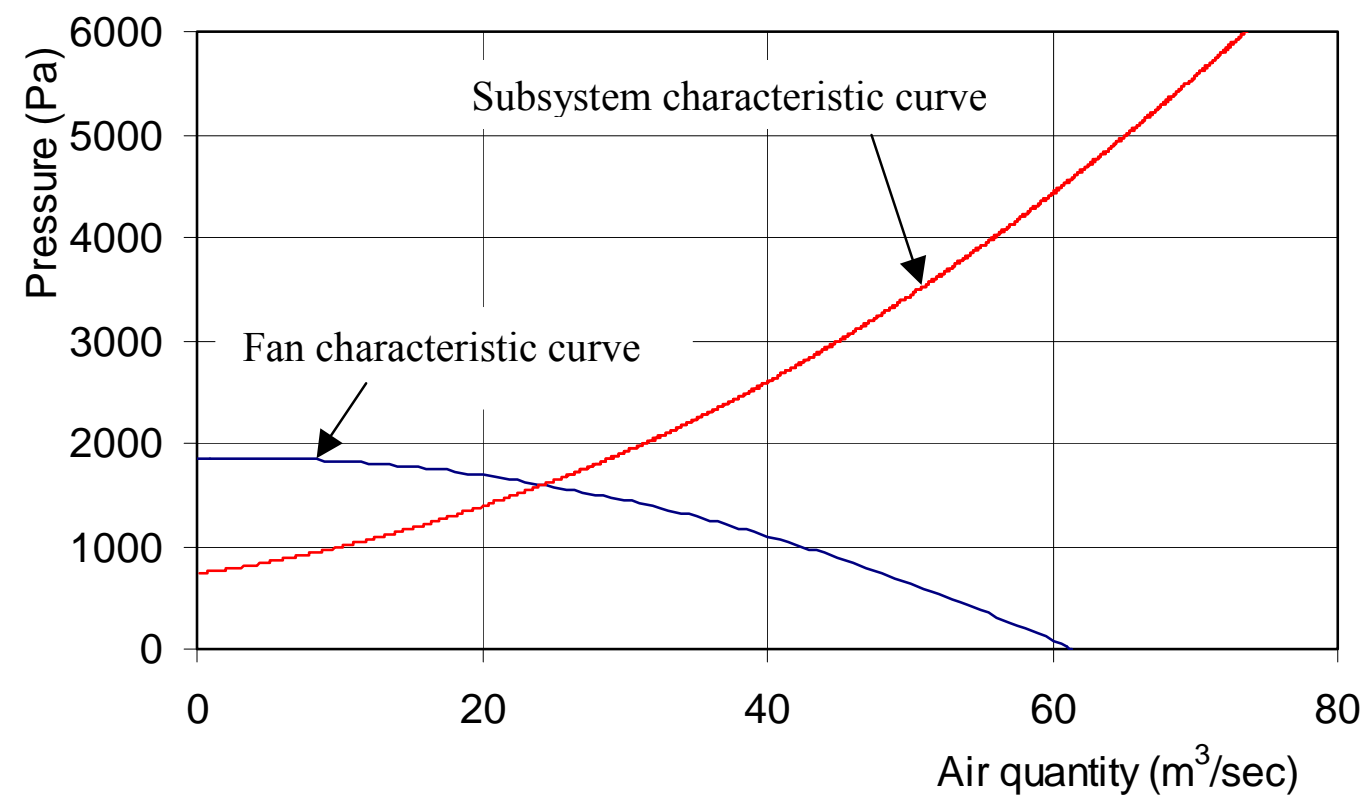

Figure 4.35 -Shape of subsystem No. 3 characteristic curve in a 2 axial and 2 centrifugal flow fans system.

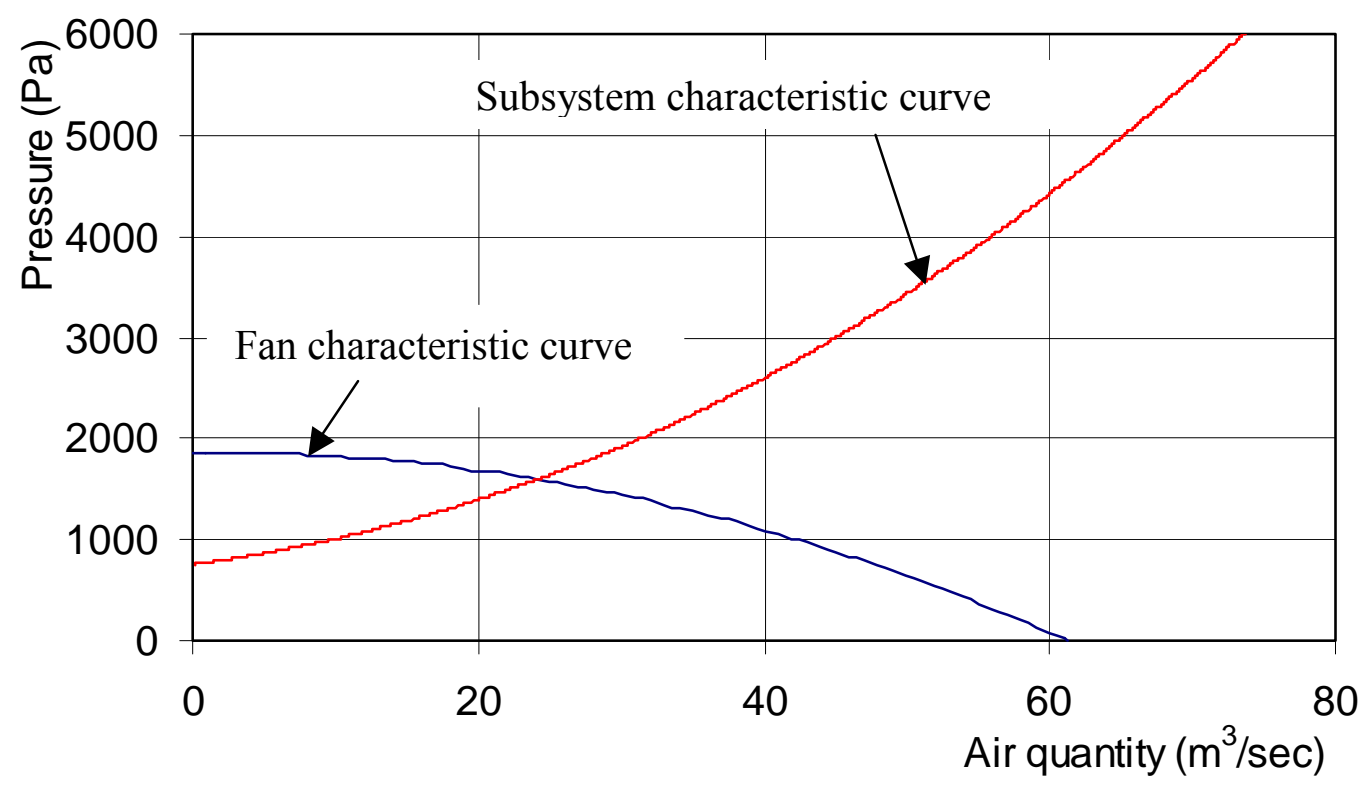

Figure 4.37 -Shape of subsystem No. 3 characteristic curve in a 1 axial and 3 centrifugal flow fans system. 


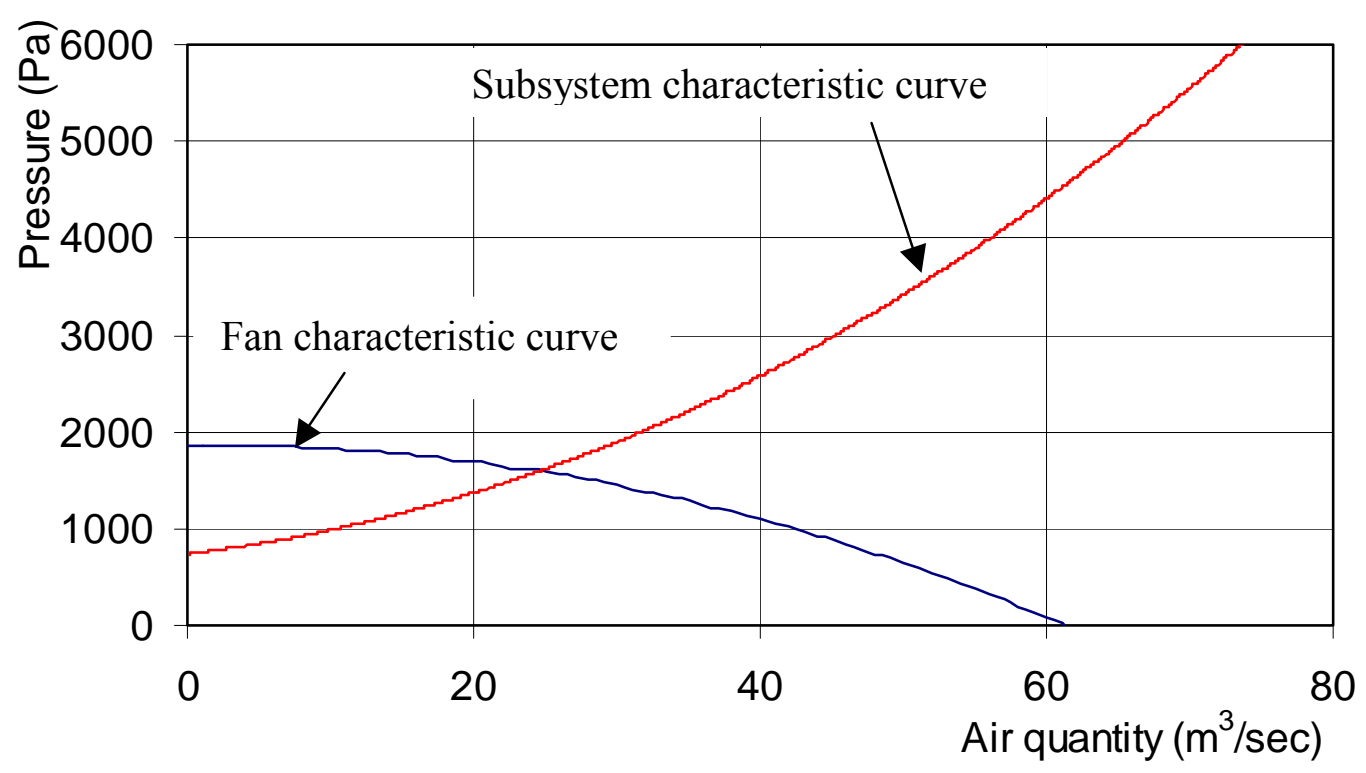

Figure 4.38 -Shape of subsystem No. 3 characteristic curve in a 4 centrifugal flow fans system.

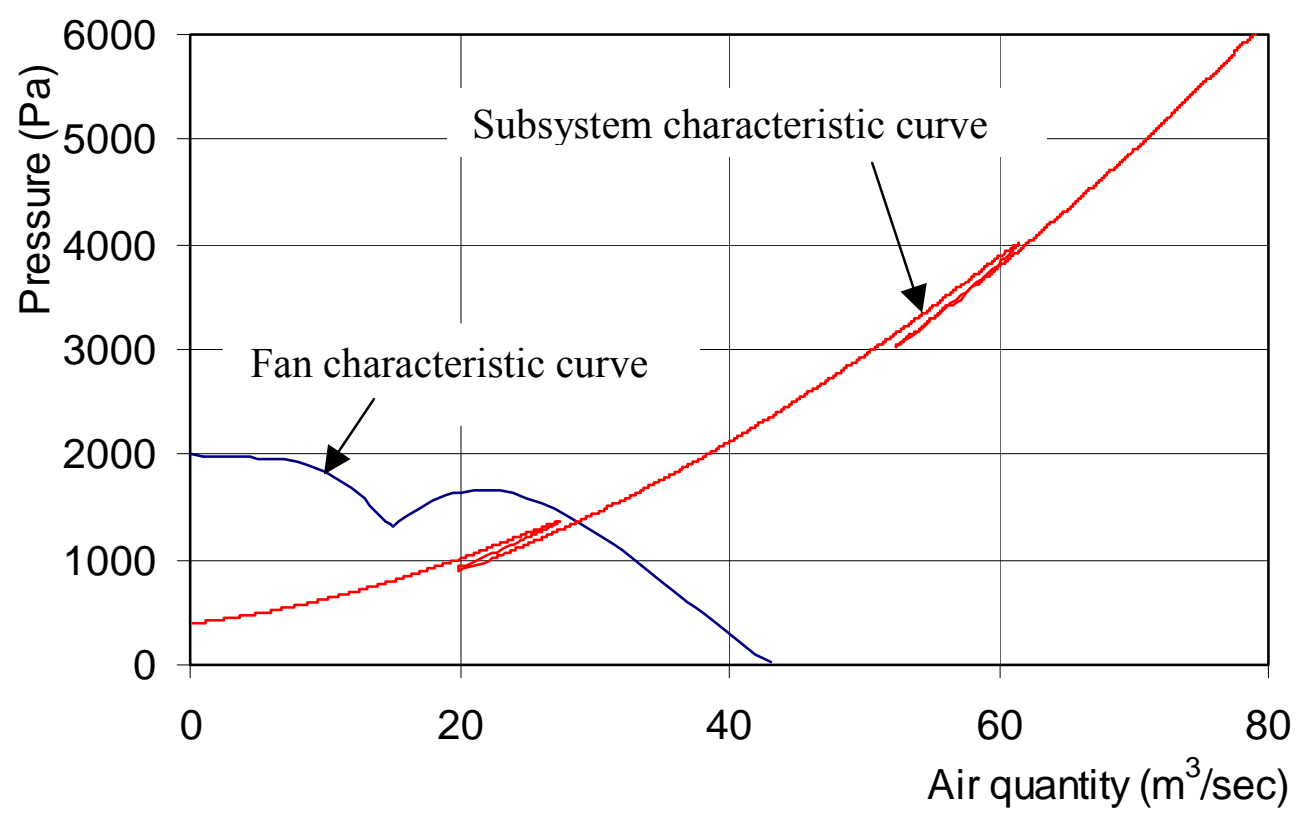

Figure 4.39-Shape of subsystem No. 4 characteristic curve in a 4 axial flow fans system. 


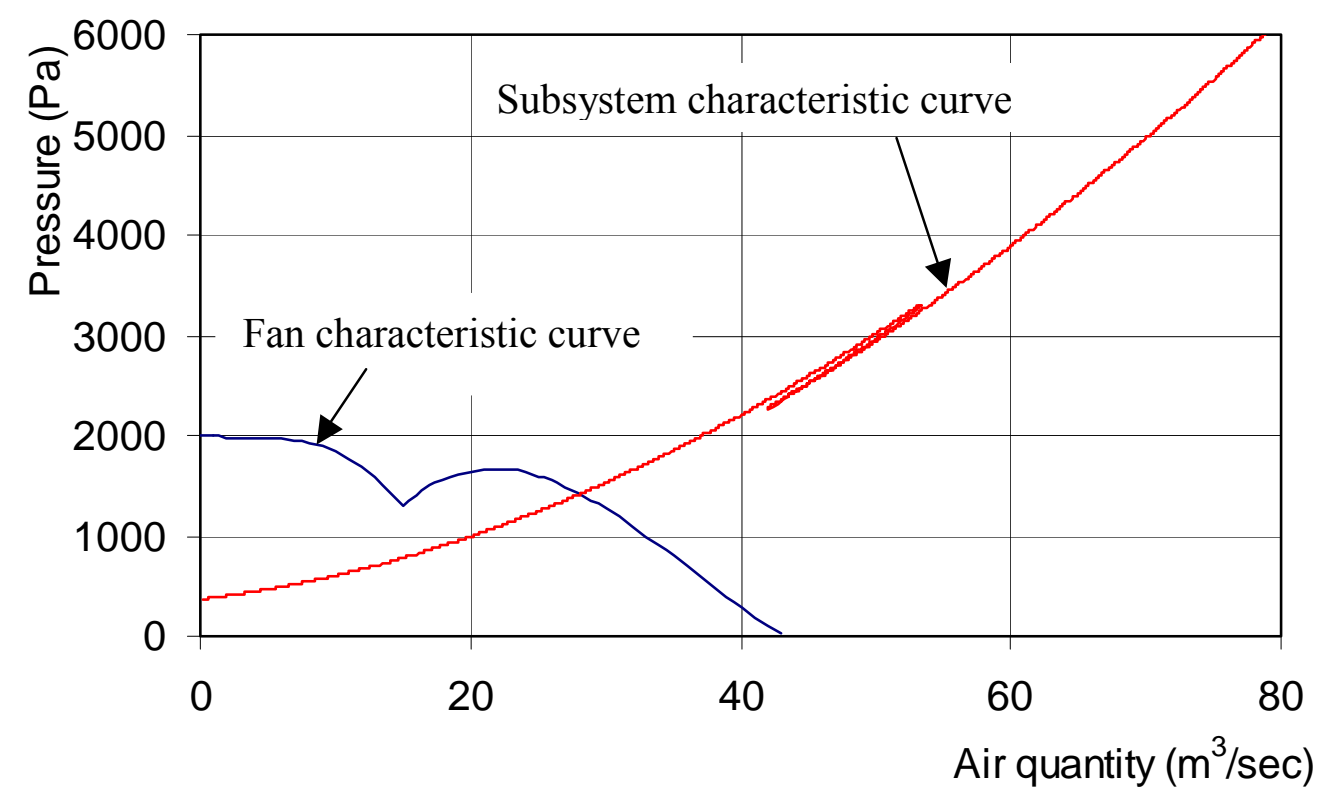

Figure 4.40 -Shape of subsystem No. 4 characteristic curve in a 3 axial and 1 centrifugal flow fans system.

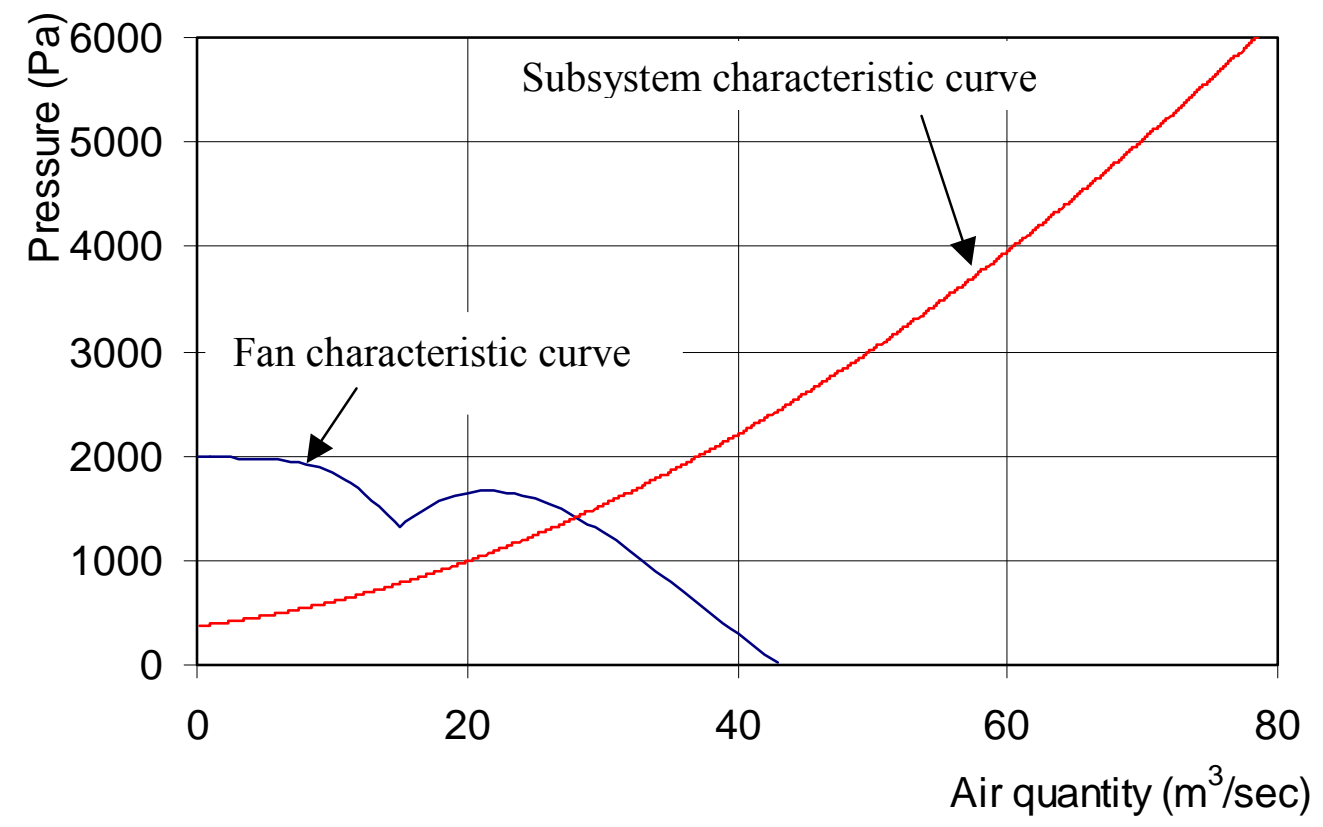

Figure 4.41 -Shape of subsystem No. 4 characteristic curve in a 2 axial and 2 centrifugal flow fans system. 


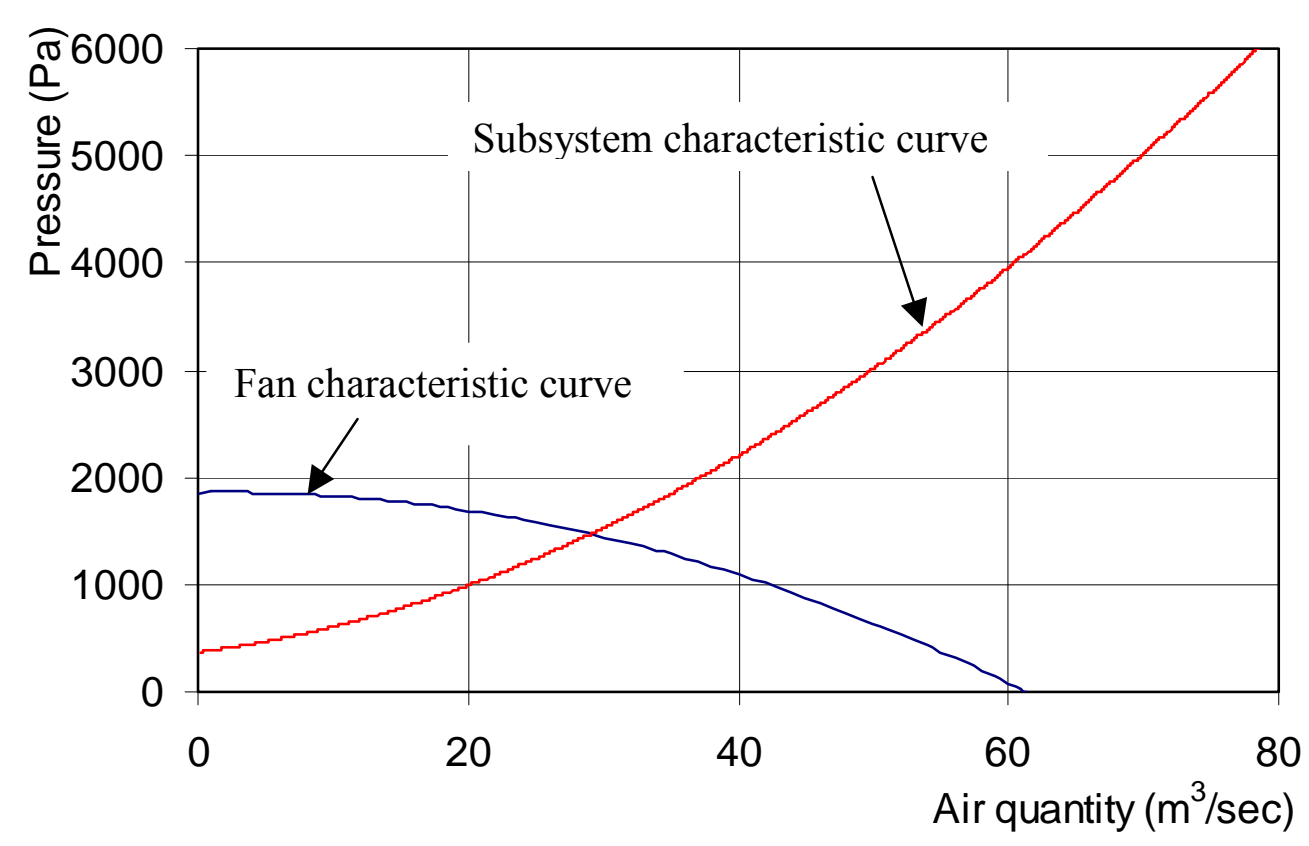

Figure 4.42 -Shape of subsystem No. 4 characteristic curve in a 1 axial and 3 centrifugal flow fans system.

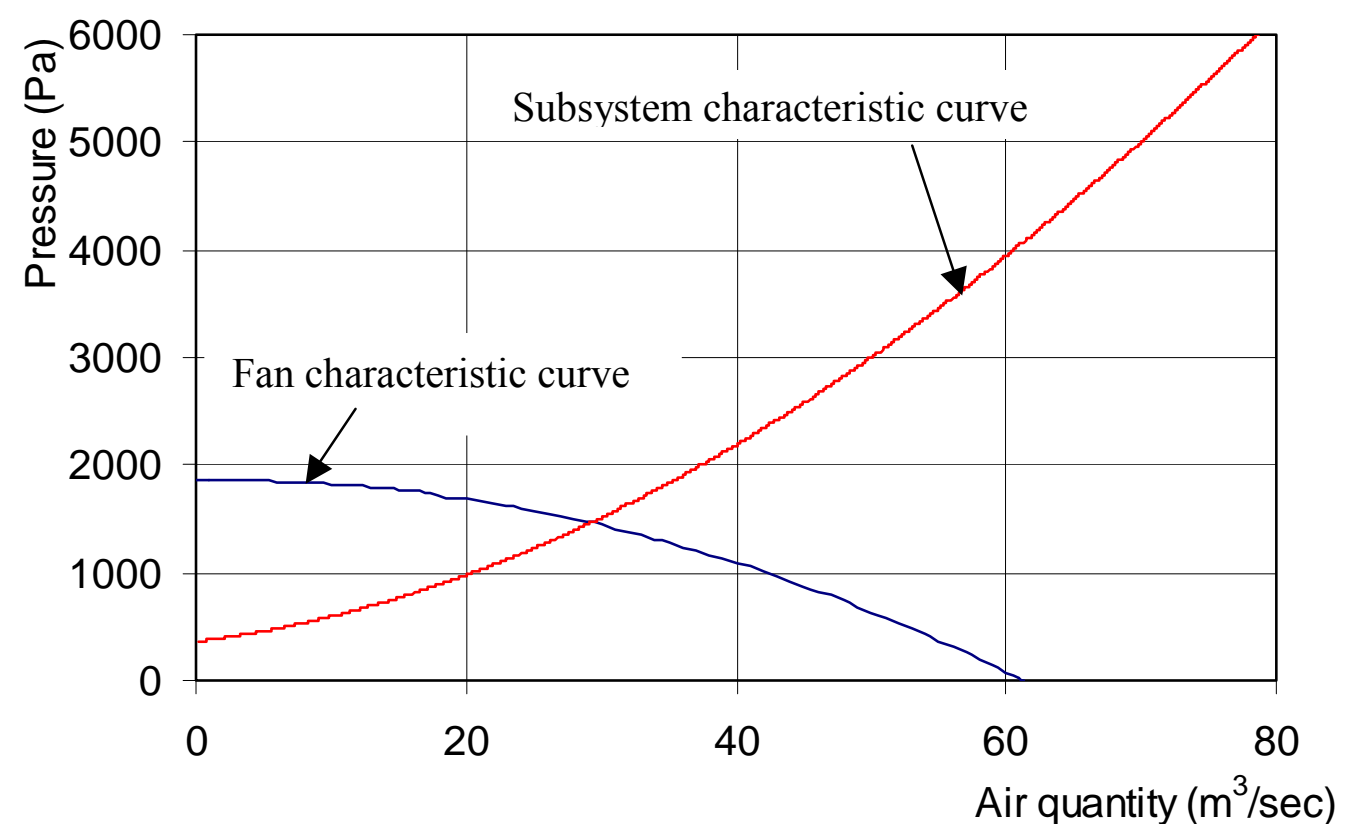

Figure 4.43 -Shape of subsystem No. 4 characteristic curve in a 4 centrifugal flow fans system. 
the speed of the fan installed in branch No. three has been changed to $120 \%$ or $80 \% \mathrm{rpm}$ of its original speed.

As far as we know, the shape or the speed of an associated fan does not affect the shape of a subsystem characteristic curve. In Figure 4.44 the shape of the subsystem characteristic curve of fan No. three does not change by changing its speed. There is only one operating point in case of $100 \% \mathrm{rpm}$ and $80 \% \mathrm{rpm}$, but in the case of $120 \% \mathrm{rpm}$ the number of operating points increases to three points. Figures 4.45 to 4.47 show this effect on subsystem 1, 2 and 4 respectively. In all subsystems shown, the effect of the change from $100 \%$ to $120 \% \mathrm{rpm}$ is larger than the change from $100 \%$ to $80 \% \mathrm{rpm}$. The subsystems at $80 \% \mathrm{rpm}$ are closer to the subsystems at $100 \% \mathrm{rpm}$ than the subsystems at $120 \% \mathrm{rpm}$. Increasing the speed of a fan inside a subsystem raising the resistance of any of the subsystems, consequently increasing the pressure value of a subsystem. There is no specific relation between the number and the location of the turning parts of the subsystem characteristic curve and the fan speed on the system. While it moves toward the direction of high air quantity (Figure 4.46), it does not have a specific trend in Figures 4.45 and 4.47. Changing the speed of a fan in the system may help one fan to work within it is stable zone, but it may force other fans in the system to operate within unstable zones.

The effect of changing the speed of fan No. 3 is not fixed over the subsystems. Changing the speed of fan No. 3 to $120 \%$ rpm from its original speed has two major effect on the whole system. While it improve the working condition of fan No. 3, by 
helping it to work in the stable zone, it forces fan No. 2 to work in its unstable zone as shown in Figure 4.44 and 4.46 respectively. The effect is very obvious on subsystem No. 2 (Figure 4.46) but this effect decreases on subsystem No. 4(Figure 4.47) and it is very feeble on subsystem No. 1 (Figure (4.45).

\subsection{Summary}

In mine ventilation, the pressure loss between the two endpoints of an airway, or a single fan network, is ordinarily expressed as a function of air quantity throughout, while the resistance is treated as a constant. However, this relation is intended for ventilation systems with a single fan and cannot be directly applied to a multiple fan network because of the effect of multiple pressures and air quantities have on fans in the network.

Regardless of the relation between the pressure and air quantity in the multiple fan system, some ventilation parameters have the same effect on the subsystem characteristic curve shape as single fan systems, while some have a different effect. Changing the fan branch's resistance, in multiple fan systems, has the same impact on its associated fan subsystem characteristic curve as in a single fan system, but it has a different influence on the other subsystems in the network. The bigger the resistance, the higher the slope of its subsystem curve, the lower the slope of the other subsystems in the network. In multiple fan systems, the effect of the resistance of a regular branch is the same as in single fan systems, decreasing the regular branch resistance reduces the subsystem pressure. 


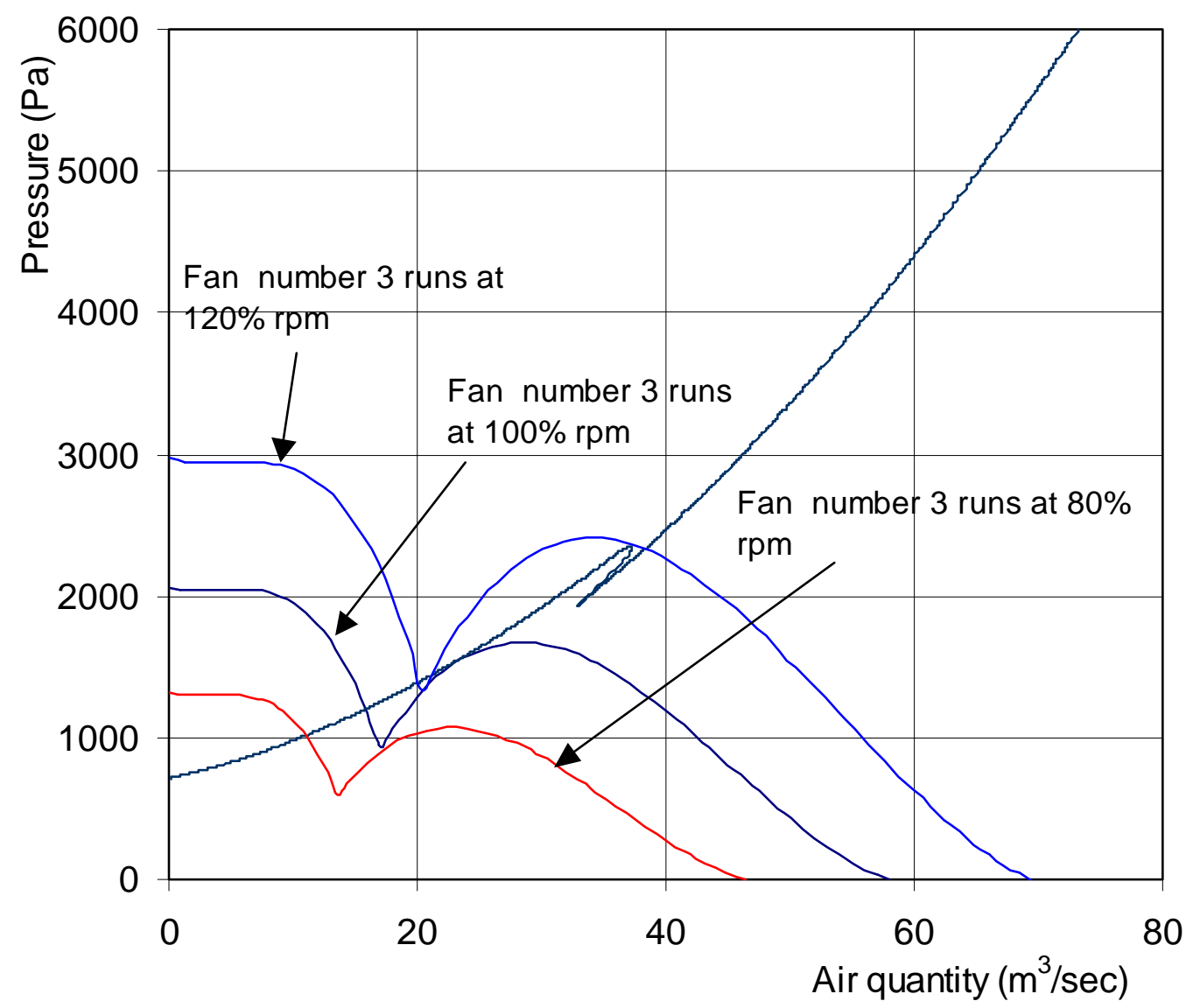

Figure 4.44 -Effect of changing the speed of fan No. 3 on its subsystem 


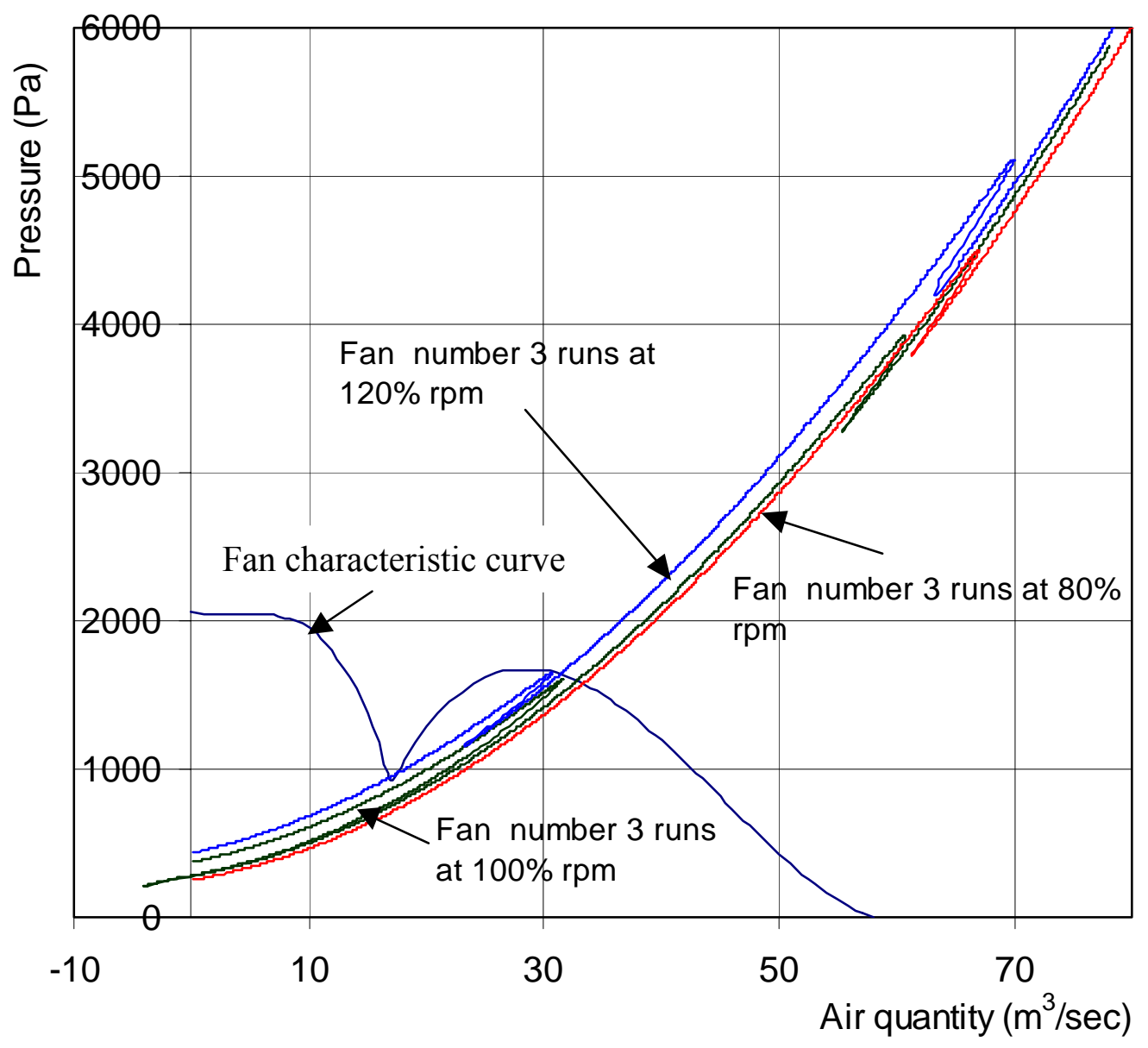

Figure 4.45 -Effect of changing the speed of fan No. 3 on the shape of Subsystem No. 1. 


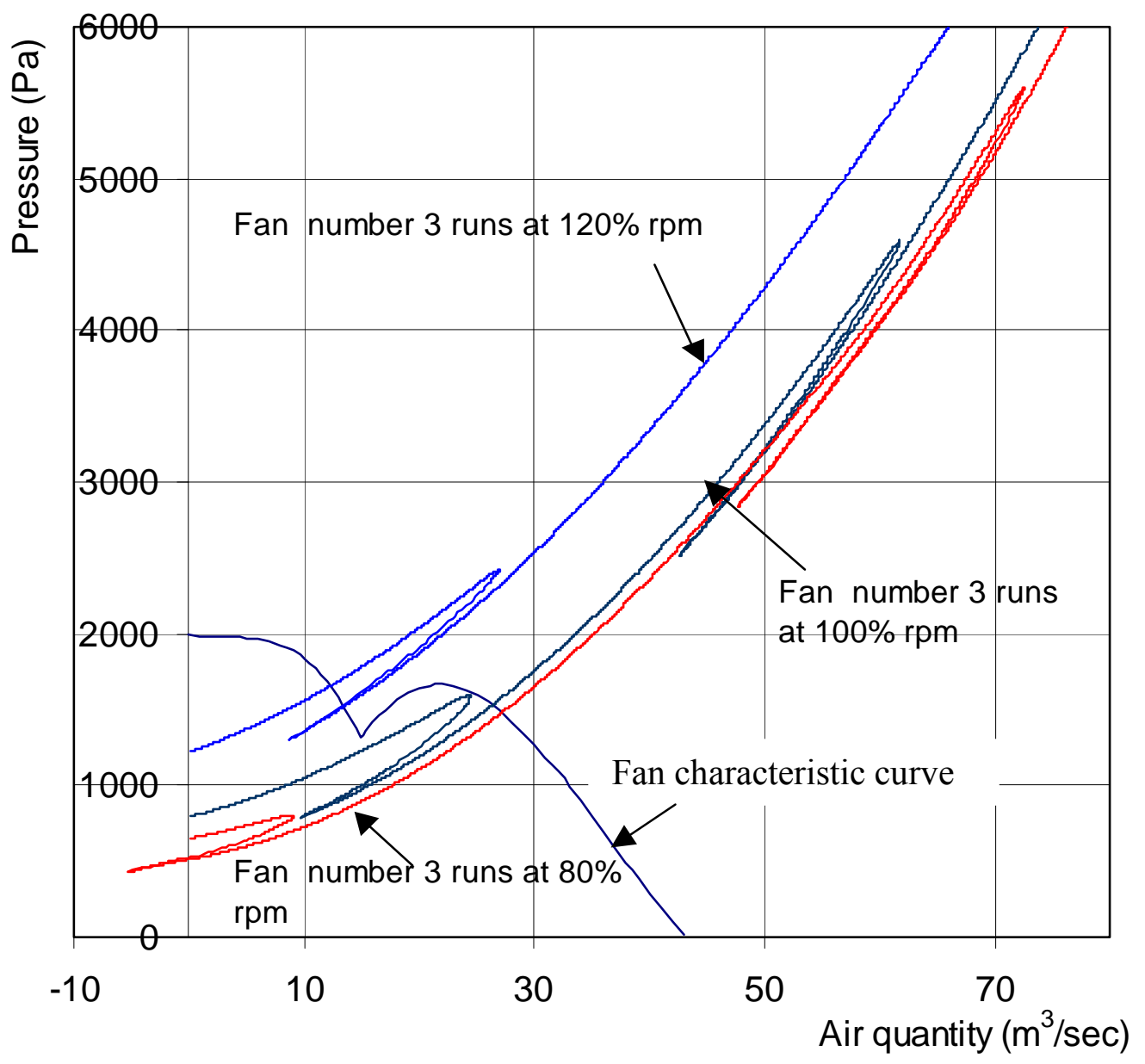

Figure 4.46-Effect of changing the speed of fan No. 3 on the shape of Subsystem No. 2. 


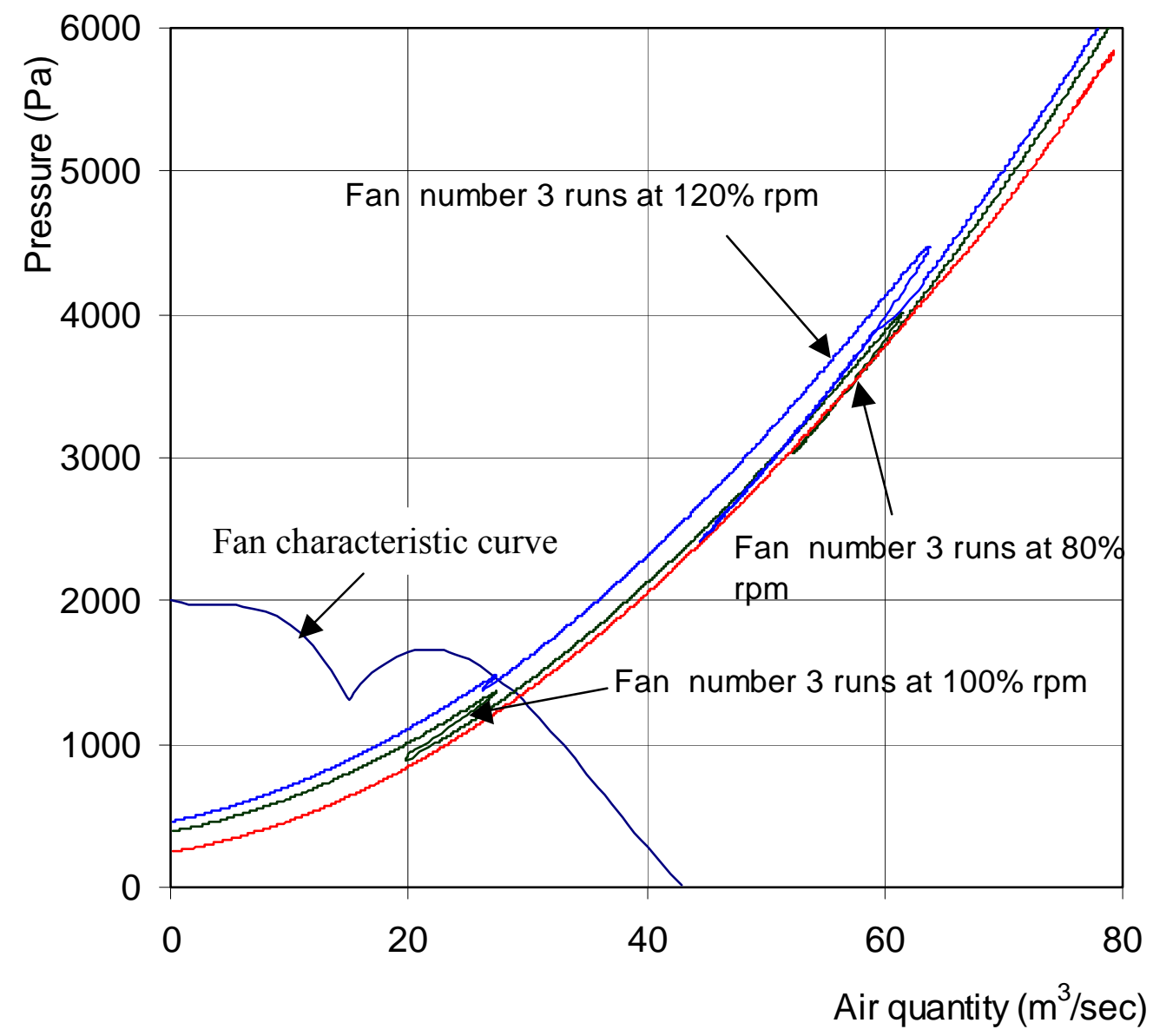

Figure 4.47-Effect of changing the speed of fan No. 3 on the shape of Subsystem No. 4. 
The influence of changing the topology of the network is considerable on the shape of the subsystem characteristic curves. The subsystem characteristic curves in multiple fan systems move toward the common mine characteristic curve of a single fan system when there is an increased number of branches and meshes in the network. This leads to a decreasing of the overall resistance, and consequently the slope of the subsystem. As a result, most of the fans in multiple fan systems work in their stable zone in the right of their stall point. The effect of fans inside any subsystem decreases; accordingly the multiple fan system becomes more stable by increasing the number of branches and meshes in the network.

Practicing the multiple fan system in industry could be dangerous if a fan fails. One or more of these situation could occur, airflow may reduce in certain sections, air may short circulate and reverse in returns from other mine sections and a flow from return airways to intake airways. Airflow direction in an airway could be reversed if fans are added to or removed from the system. The more fans in the system, the more turns in the subsystem, consequently the more instability conditions. Decreasing the number of fans can reduce the overall pressure requirements of the system.

Not only the number of fans in the system has an effect on the shape of the subsystems but also the type and speed of fans inside it. Decreasing the number of axial flow fans in the system increasing the stability of the subsystems. Increasing the speed of a fan in the system raising the resistance of any of the subsystems, consequently enlarge the pressure value at the same airflow. Relationship between fans in a multiple 
fan systems is transferable, changing the speed of a fan in the system may help one fan to work within it is stable zone, but it may force the other fans in the system to operate in their unstable zone. 


\section{CHAPTER 5 \\ PRACTICAL MULTIPLE-FANS IN REAL COAL MINES}

\subsection{Introduction}

The mining of coal underground has historically been recognized as one of the more hazardous occupations in the world. It is a universally recognized principle of underground coal mine safety that there be proper ventilation of the mine. Indeed, no aspect of safety in underground coal mines is more fundamental than proper ventilation. A basic tenet of mining safety states that ventilation must be sufficient: (1) To dilute, render harmless and carry away the hazardous components of mine air, such as potentially explosive methane; and (2) to provide necessary levels of oxygen to the miners' working environment.

Many mines produced dangerous gases - methane and coal gas. Very fine coal dust could be highly explosive and a spark could result in a fatal explosion. When methane (CH4) was mixed with oxygen it was called "fire damp" and was very dangerous. Other gases, including carbon monoxide, called "white damp" by miners, or "black damp" which was a mixture of air and carbon dioxide could accumulate in mines and be poisonous to the miners. Breathing fine coal dust over many years could lead to a disease known as pneumoconiosis, or "black lung." The disease progresses by causing coughing and minor lung impairment to progressive loss of lung capacity, increasing disability and possibly death.

The mines require very effective ventilation systems to keep the levels of explosive gases to a safe level and to make sure there is a fresh air for the miners to 
breath. Most mines have large ventilation fans built at the surface which are used to draw air out of the mines. Special shafts are dug to permit fresh air to enter the workings. Booster auxiliary fans are used to force air further into the workings. The number of fans in the mine depends upon the ventilation requirements and mine size.

In this chapter, three coal mines, A, B and C, have been used to study the shape of the subsystems characteristic curves in real mines. A multiple fan system has been applied to each of them. Mine A is the smallest, and has 3 axial flow fans. Mine B, has 4 fans; one of them is a centrifugal. Mine $\mathrm{C}$, which is the largest, has 5 axial flow fans: One centrifugal fan and four axial flow fans are used in this mine. The axial flow fans types are Jeffrey Joy M120-65, 8HUA96, Jeffrey 8HU-117, and Joy H-120-65D. The characteristic curves at different blade angles, with operating points for the first two types, are provided, as shown in Figure 5.1 and 5.2 respectively. The characteristic curves, for the last two types, at two different blade angles with the operating points are provided. The centrifugal fan installed in mine B, shown in Figure 5.3, is Zurn/Clarage 153 AFM-5350 SWSI.

For safety reasons, fan manufacturers do not provide the ventilation engineers with the complete axial flow fan characteristic curves. Thus the stable part of the curves is available and they are shown as provided from the manufacture in Figures 5.1 to 5.3. Depending upon these stable parts the whole curves can be deduced (Ramani, 2001, and Mousset-Jones, 2001). 


Joy M120-65-880 Axial Vane
880 RPM@0.075 lb/cu.ft.w/Standard Exhaust Evase

Pressure type: Static

Fan curve date:04/01/82

Fan setting: Blades tip angle

Fan drawing \#: C-8231

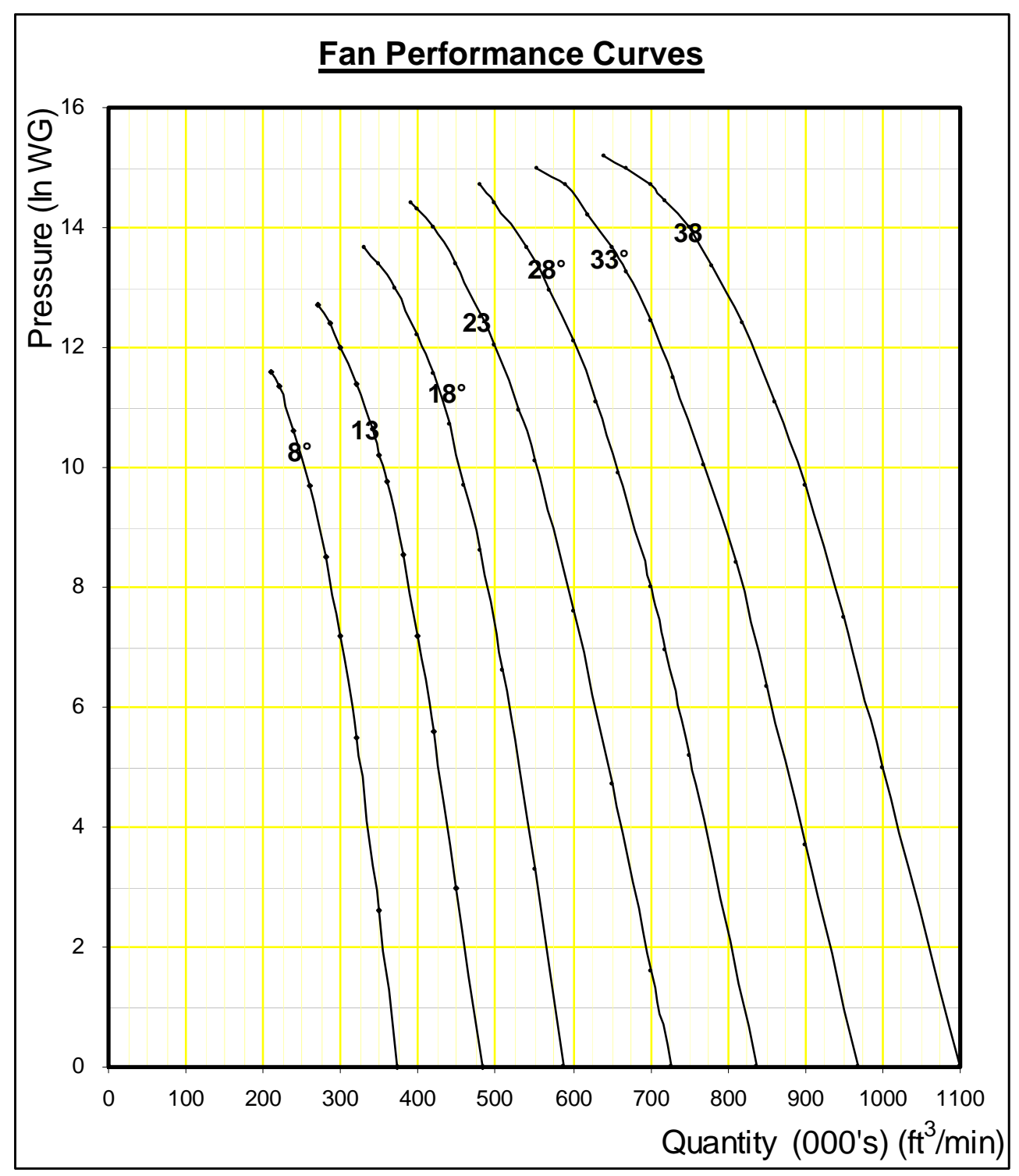

Figure 5.1 -Fan performance curve for Joy M120-65 at different blade angles. 
Ventcad fan ID:1217

Pressure type: Static

Fan curve date: $07 / 16 / 74$

Fan settings: Blade / Socket hole positions Fan drawing \#: 194A037

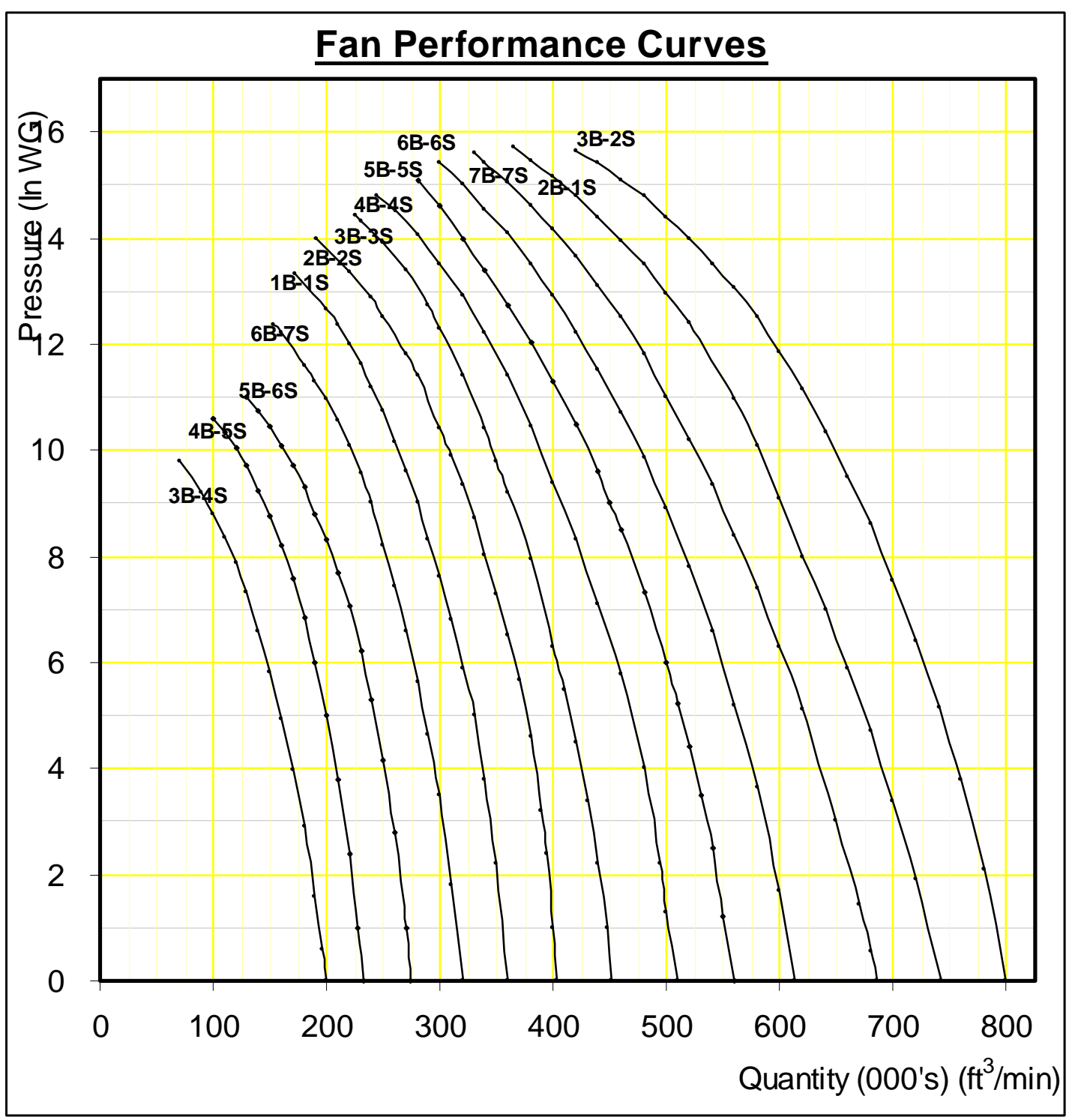

Figure 5.2 -Fan performance curve for Jeffrey 8HUA96 at different blade angles 
Zurn/Clarage 153 AFM-5350 SWSI Centrifugal 878 RPM@ 0.0721 lb/cu.ft. w/Standard Exhaust Evase

Ventcad fan ID:2606

Pressure type: Static

Fan settings: Louver positions (\%) Fan drawing \#: RLF-01407

Comments/Notes: SP includes silencer loss of 0.96"@ O-point

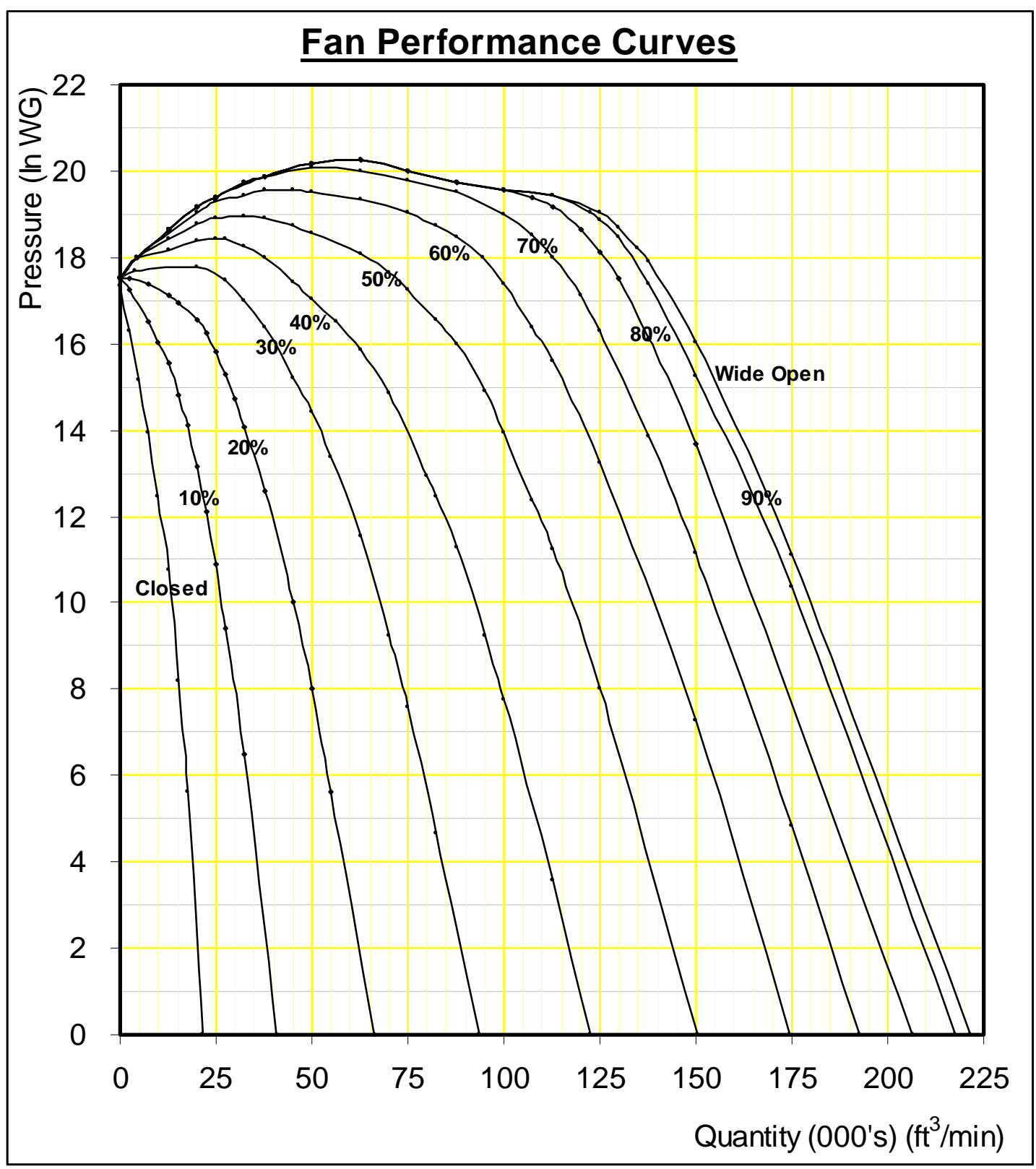

Figure 5.3 -Fan performance curve for Zurn/Clarage 153 AFM-5350 SWSI Centrifugal at different blade angles 


\subsection{Ventilation Performance in Mine A}

Mine A is located in the Herrin No. 6 coal seam. The mine consists of two longwall and three continuous mining units. The mine is accessed at two portals. Both of them are used as man and material shafts, but only one of them works as a production shaft. Airflow is drawn into the mine through five intake shafts by surface mounted exhausting axial flow fans installed at three return air shafts (Pompei, at el., 1998). Table 5.1 shows the operating pressure and air quantity that were measured on October 1998 for each fan. The fans exhausted a combined total of $524.72 \mathrm{~m}^{3} / \mathrm{sec}$ from the mine. In addition, the pressures throughout the mine and pressure differentials between air courses were measured. From this data, a balanced single line schematic of the mine was developed as shown in Appendix A.1. In this figure, airflow direction, air quantities (in $100,000 \mathrm{cfm}$ ) and ventilation pressures (in inches water gauge) are indicated.

Table 5.1 Main mine fan's quantities and total pressures for mine A

\begin{tabular}{|c|c|c|c|c|c|c|}
\hline \multirow{2}{*}{ Fan \# } & $\begin{array}{c}\text { Measured } \\
\text { Air } \\
\text { Quantity, } \\
\mathbf{m}^{\mathbf{3}} / \mathbf{s e c}\end{array}$ & $\begin{array}{c}\text { Calculated } \\
\text { Air } \\
\text { Quantity, } \\
\mathbf{m}^{\mathbf{3}} / \mathbf{s e c}\end{array}$ & Difference & $\begin{array}{c}\text { Measured } \\
\text { Pressure, } \\
\text { Pa }\end{array}$ & $\begin{array}{c}\text { Calculated } \\
\text { Pressure, } \\
\text { Pa }\end{array}$ & Difference \\
\hline 1 & 164.00 & 160.00 & 4.00 & $1,805.89$ & $1,953.33$ & -147.44 \\
\hline 2 & 199.53 & 194.00 & 5.53 & $1,631.53$ & $1,825.45$ & -193.92 \\
\hline 3 & 161.19 & 160.00 & 1.19 & $2,047.51$ & $2,002.71$ & 44.80 \\
\hline
\end{tabular}


From a mine ventilation viewpoint, mine A represents the largest network of the three mines. Its network, after solving the simple parallel-series connections, consists of 326 branches, 217 nodes and 110 meshes. The Jacobian matrix of this network is too big. The modified damped Newton methods takes more than 5 days to converge upon the first point in the solution curve of subsystem No. 1. On the other hand, the modified Hardy Cross method takes about 35 minutes to draw a complete subsystem curve.

The three axial flow mine fans installed in this mine are the Jeffrey 8HUA96 type. As shown in Figure 5.4, fan No. 1 operates at 1,245 rpm with a blade angle 2B-2S. The stable part of the fan characteristic curve that is provided from the fan manufacturer is indicated by a dotted line. The rest of the curve is assumed as shown as a dotted part of the fan characteristic curve in Figures 5.5 - 5.7. Subsystem characteristic curve for fan No. 1 is a strictly increasing function. It does not have any fluctuation but it does not pass through the origin. The same can be written about the subsystems of fans No. 2 and 3 Figures 5.5 and 5.6, respectively. Fan No. 2 runs at 1,230 rpm at a blade angle between 3B-3S and 4B-4S and fan No. 3 runs at 1,185 rpm at a blade angle 2B-2S. Table 5.1 shows the actual operating point measured at each fan and the operating point obtained by applying the modified Hardy Cross algorithm to the mine ventilation network of this mine. The differences between the measured and calculated operating points are fairly small. 


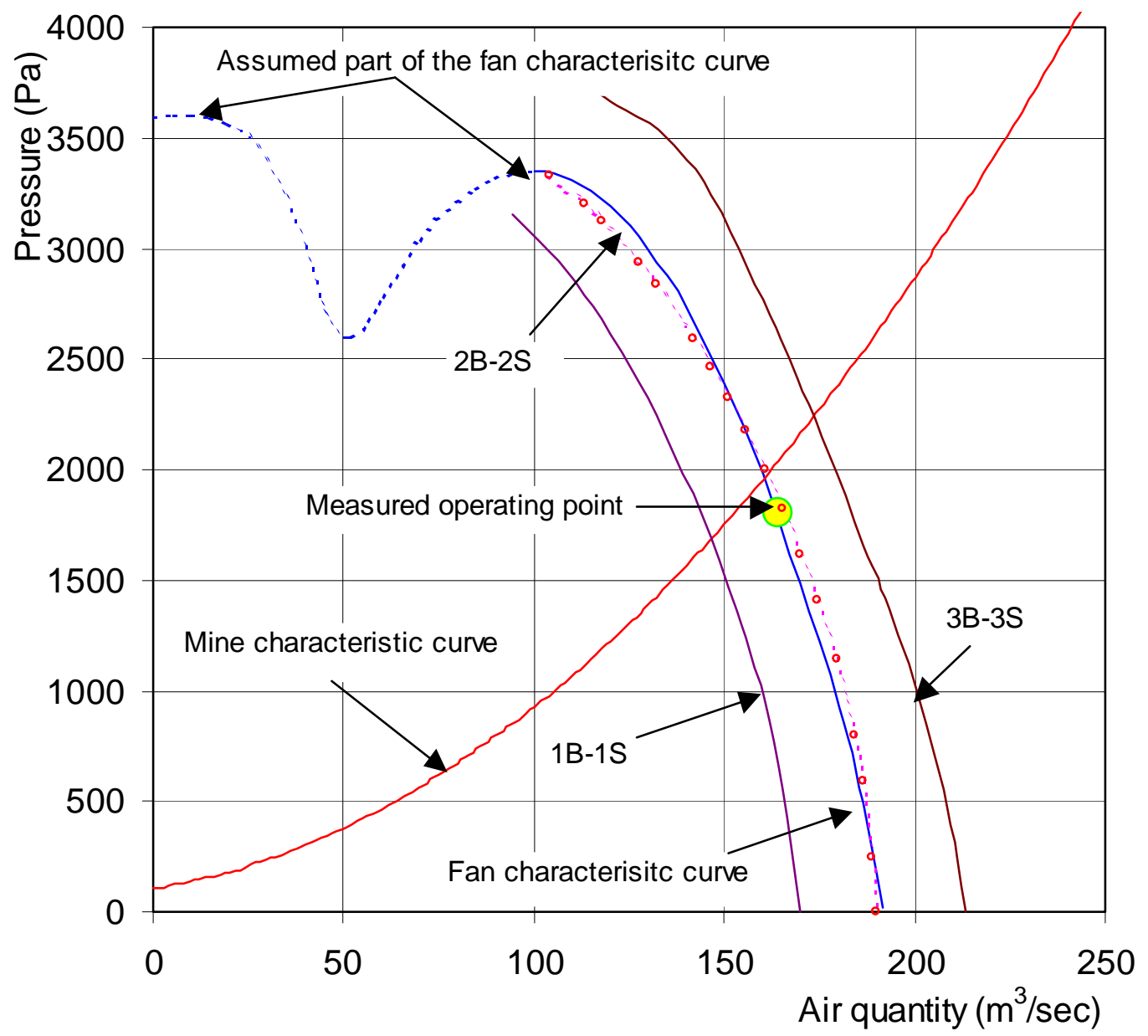

Figure 5.4 -Subsystem No. 1 characteristic curve (Mine A) 


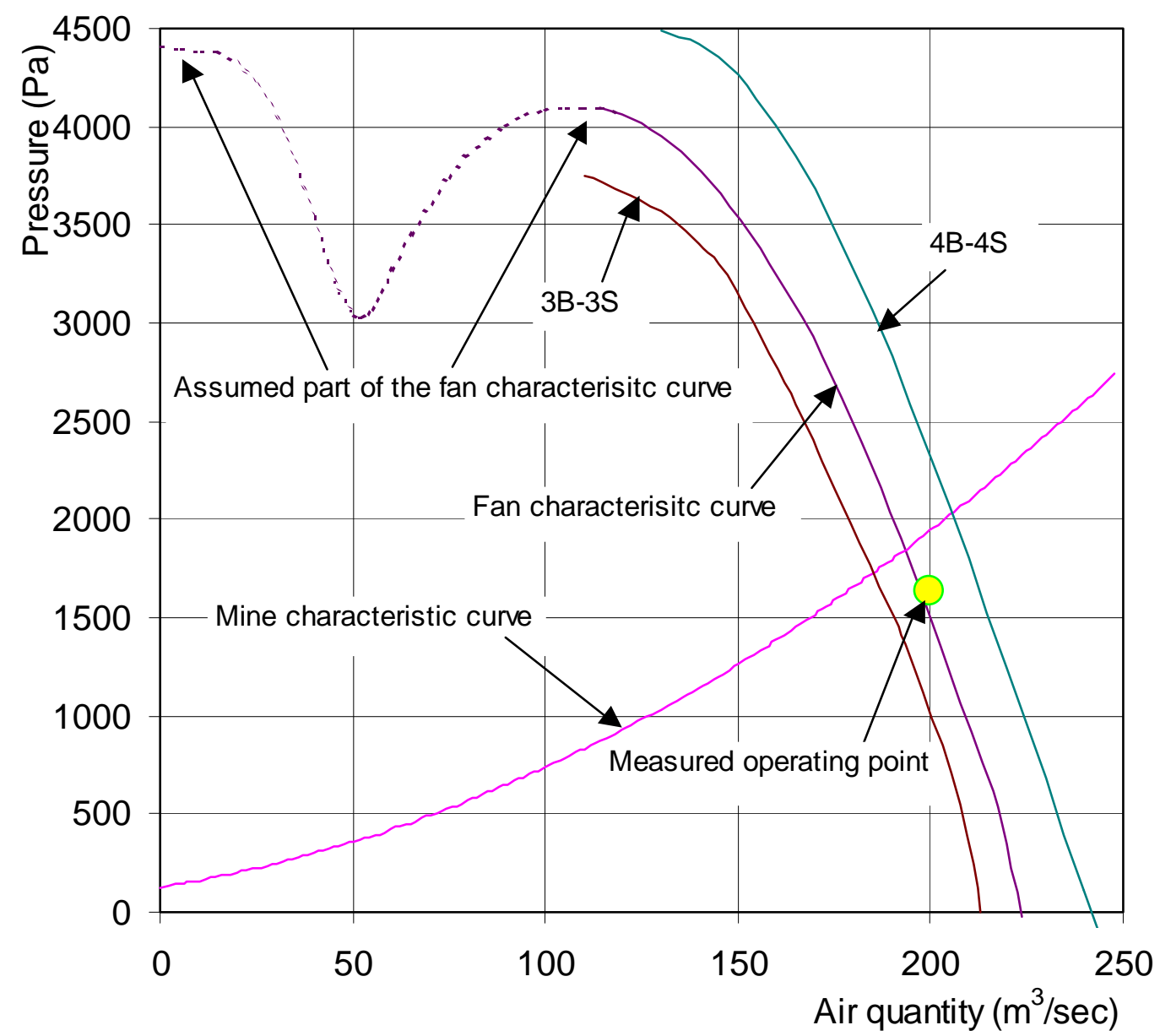

Figure 5.5 -Subsystem No. 2 characteristic curve (Mine A) 


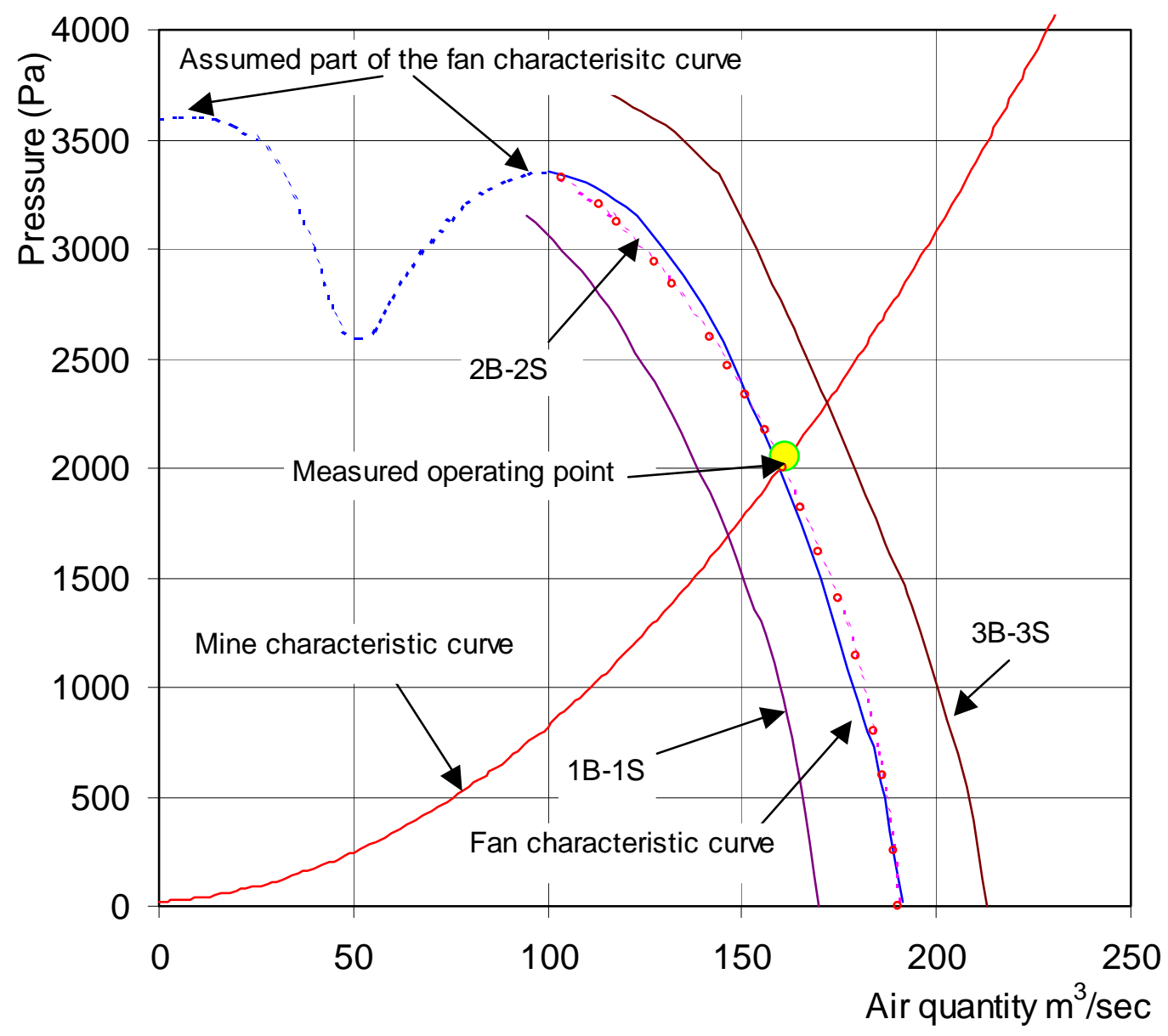

Figure 5.6 - Subsystem No. 3 characteristic curve (Mine A) 


\subsection{Ventilation Performance in Mine B}

Mine B is located in the Pittsburgh No. 8 coal seam. The mine has five active continuous mining sections and one active longwall section. The active area of the mine contains three gob areas, the north longwall gob, the south longwall gob, and the $8 \mathrm{~A}, \mathrm{~B}$ and $\mathrm{C}$ longwall panels. Three axial and one centrifugal flow surface exhausted mine fans, installed at four return air shafts, have been used to ventilate this mine. On February 1998 each fan's air quantity and total pressure were measured as shown in Table 5.2 (Schroeder et al., 1998). Appendix A. 2 shows a balanced single line schematic of the portion of the mine under investigation. The air quantity and its direction at each branch, in cfm, and the pressure difference at each node, in inch of water gauge, are indicated in the figure.

Mine B consists of 319 branches and 200 nodes. The number of fundamental meshes is $(M)=\left(n_{b}-n_{n}+1\right)=319-200+1=120$ meshes. Applying the modified damped Newton method still cannot solve a network of this size, but the modified Hardy Cross method is successful in tracing the four subsystems. Three axial flow type M120-65 Joy axivane mine fans are installed in this mine. The fourth fan is TC Clarage centrifugal fan. Fan No. 1 (Figure 5.7) runs at a speed of $901 \mathrm{rpm}$ at a blade angle between 18 and 23 degree. Fan No. 2 runs at 902 rpm between 13-18 degree, fan No. 3 runs at 902 rpm, but the chart available shows the blade angle between 8 - 18 degrees as shown in Figures 5.8 and 5.9, respectively. The centrifugal fan, fan No. 4, runs at a speed of $891 \mathrm{rpm}$ between blade angle $70-80 \%$ as shown in Figure 5.10. All the subsystems characteristic curves 
pass through the origin and do not have any zigzag parts; they all look like a single fan system. Table 5.2 shows the difference between the measured and the calculated operating point values. The different airflow values are not large but there is a big difference between the pressure values. Subsystem No. two shows the biggest difference in measured and calculated pressure, around 617.76 Pascal. These divergences may result from the approximation of the fan characteristic curves.

Table 5.2 Main mine fan's quantities and total pressures for mine B

\begin{tabular}{|c|c|c|c||c|c|c|}
\hline \multirow{2}{*}{ Fan \# } & $\begin{array}{c}\text { Measured } \\
\text { Air } \\
\text { Quantity, } \\
\mathbf{m}^{\mathbf{3} / \mathbf{s e c}}\end{array}$ & $\begin{array}{c}\text { Calculated } \\
\text { Air } \\
\text { Quantity, } \\
\mathbf{m}^{\mathbf{3} / \mathbf{s e c}}\end{array}$ & Difference & $\begin{array}{c}\text { Measured } \\
\text { Pressure, } \\
\text { Pa }\end{array}$ & $\begin{array}{c}\text { Calculated } \\
\text { Pressure, } \\
\text { Pa }\end{array}$ & Difference \\
\hline 1 & 272.39 & 255.00 & 17.39 & $1,665.16$ & $1,982.54$ & -317.38 \\
\hline 2 & 208.71 & 185.00 & 23.71 & $1,837.03$ & $2,454.79$ & -617.76 \\
\hline 3 & 167.90 & 161.00 & 6.9 & $1,063.61$ & $1,181.00$ & -117.39 \\
\hline 4 & 79.03 & 75.00 & 4.03 & $2,283.40$ & $2,733.25$ & -449.85 \\
\hline
\end{tabular}




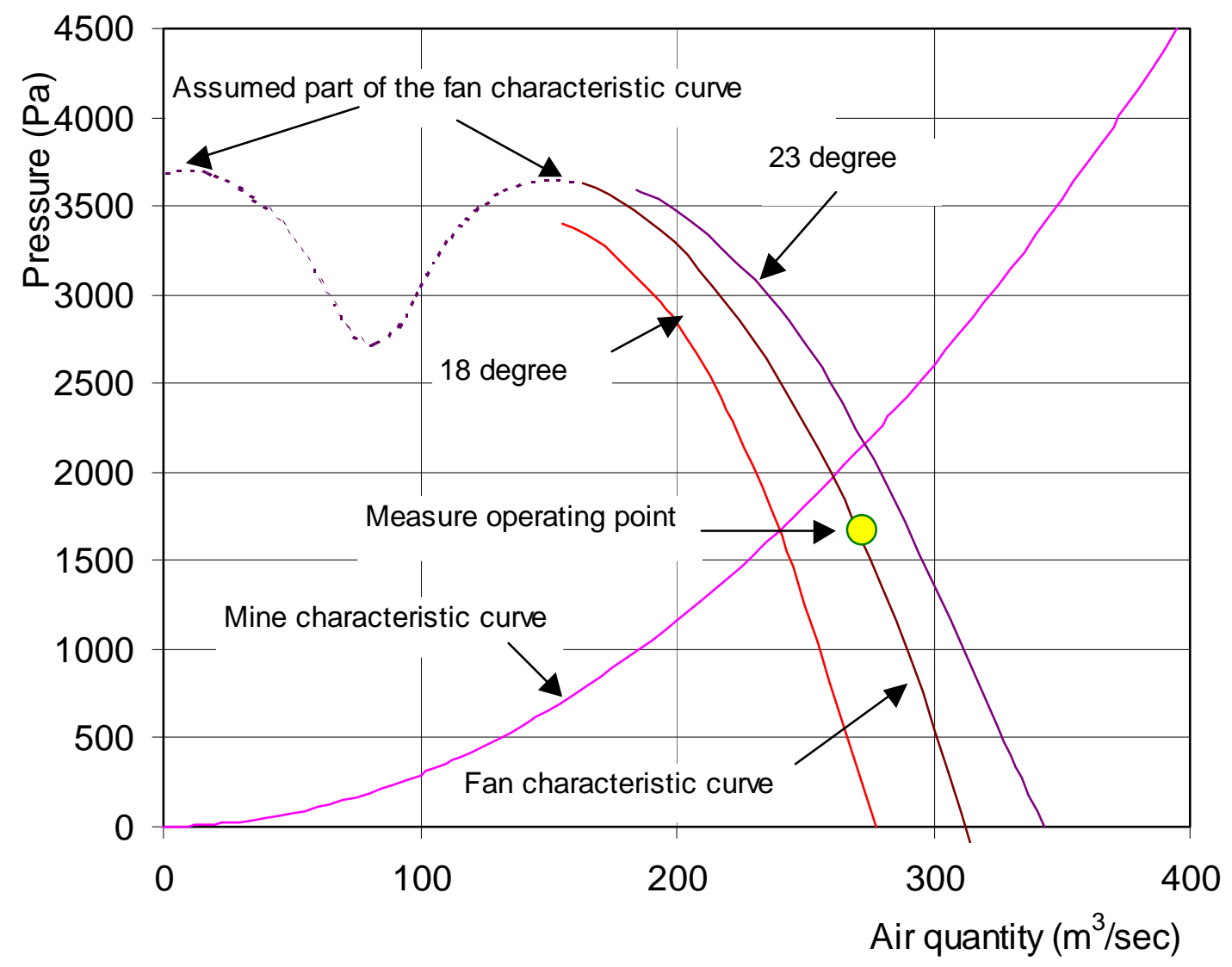

Figure 5.7 - Subsystem No. 1 characteristic curve (Mine B) 


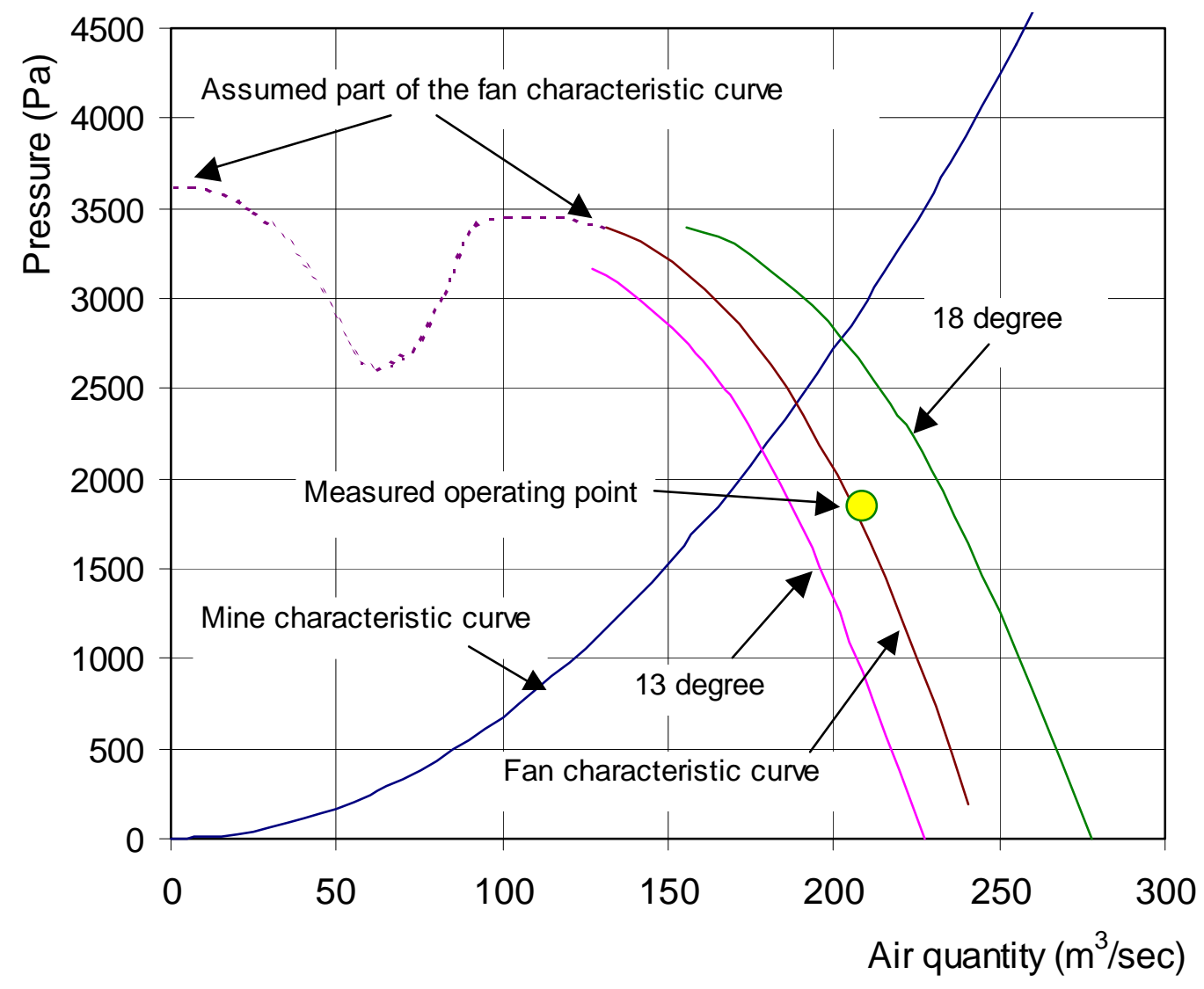

Figure 5.8 -Subsystem No. 2 characteristic curve (Mine B) 


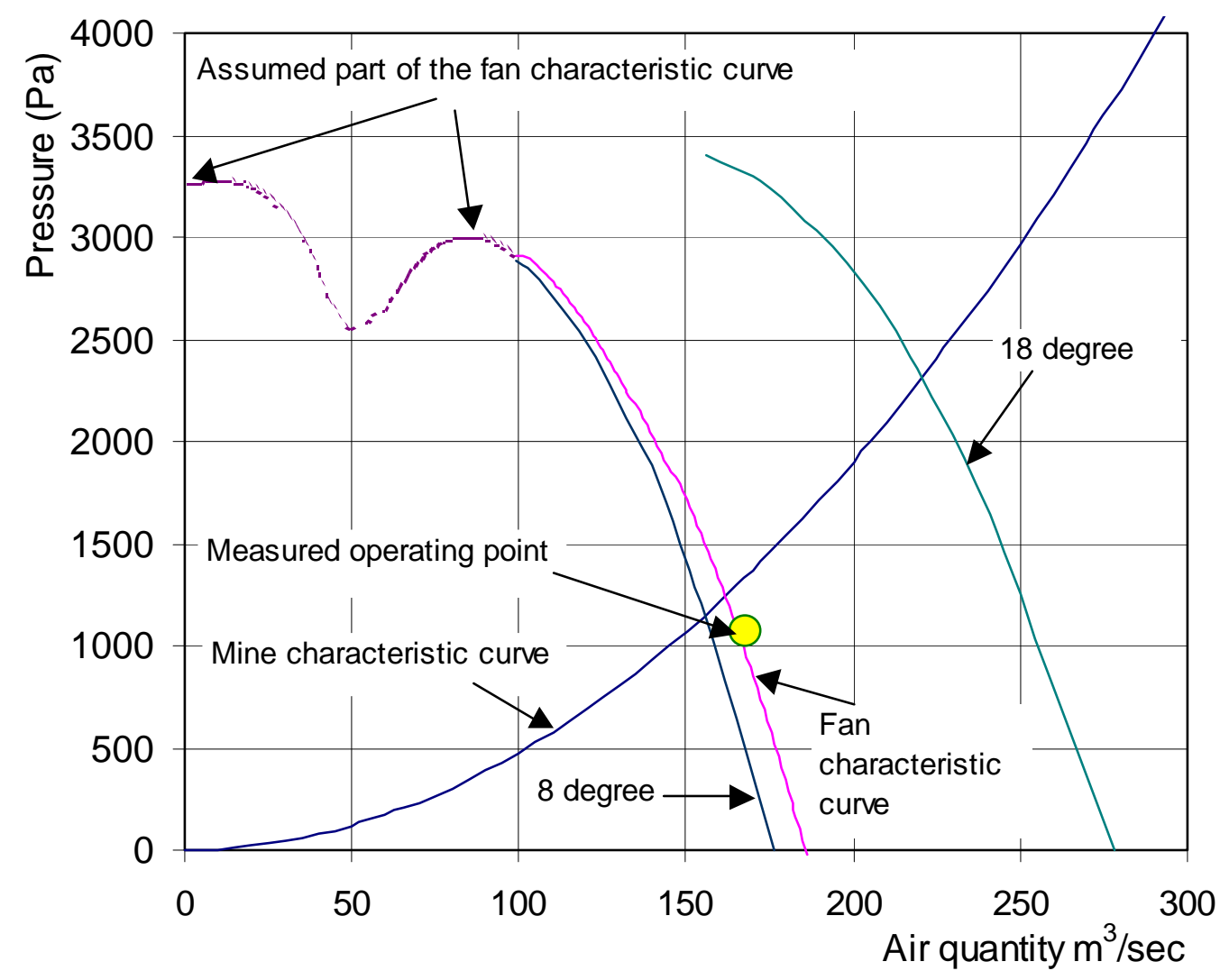

Figure 5.9 -Subsystem No. 3 characteristic curve (Mine B) 


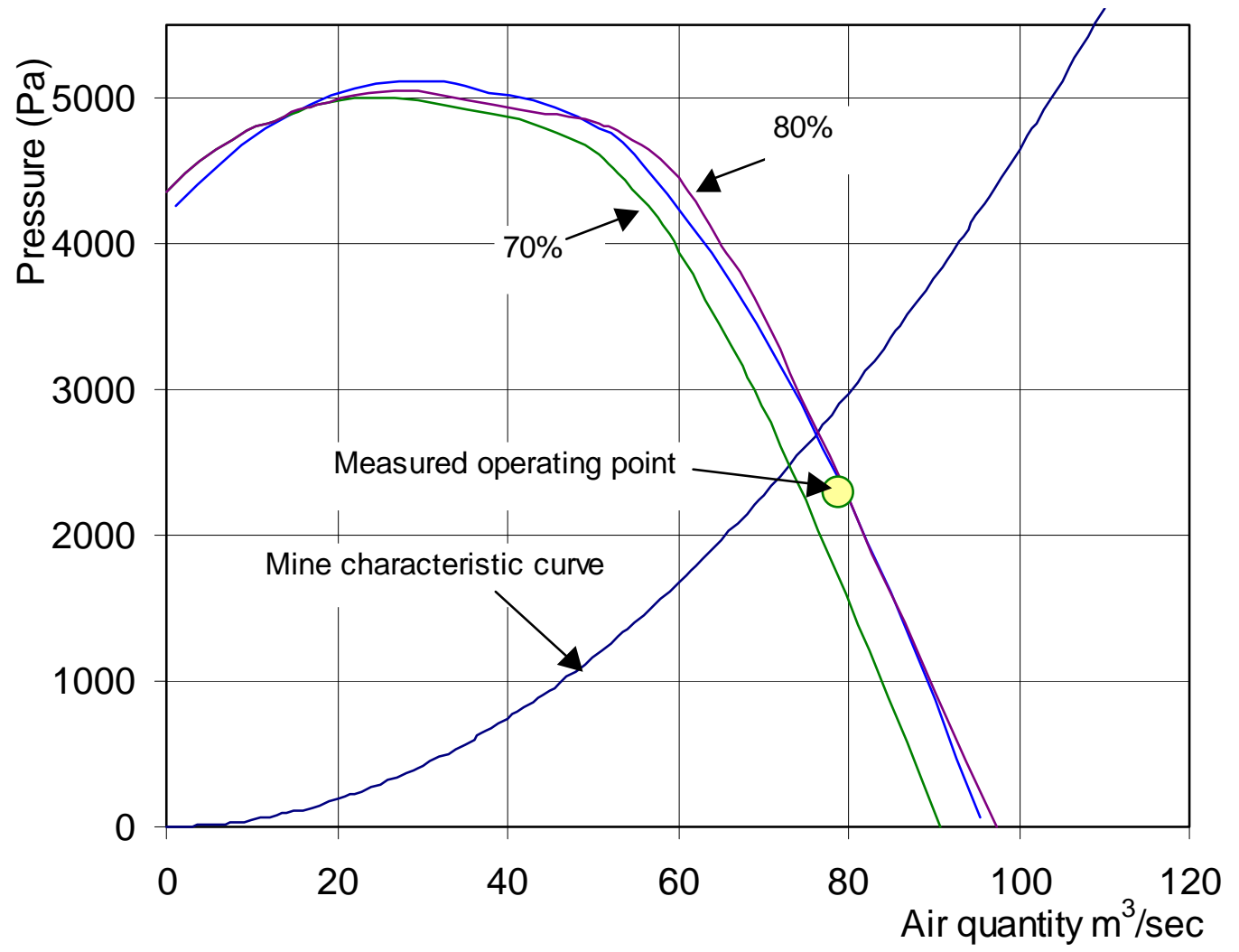

Figure 5.10 -Subsystem No. 4 characteristic curve (Mine B) 


\subsection{Ventilation Performance in Mine C}

This mine has an explosion history. On July 9, 1997, an explosion occurred in the sealed area of the southeastern portion of the mine. This was the third explosion to date in this area (Urosek, 1997). The southeastern portion of the mine was sealed from the rest of the mine by closing $1^{\text {st }}$ east, $2^{\text {nd }}$ east and $3^{\text {rd }}$ east (Appendix A. 3 ).

Five surface exhaust axial flow mine fans installed at five return air shafts have been used to ventilate this mine. Fans installed at shafts 1, 2, 4, and 5 are type Joy H-12065D but the fan installed in shaft No. 3 is Jeffrey $8 \mathrm{HU}-117$. The network of this mine consists of 99 branches, 53 nodes and 47 meshes. Both the modified Hardy Cross and the modified damped Newton methods were successful in obtaining the subsystem characteristic curves for the five fans in this mine. The modified damped Newton is much slower than the modified Hardy Cross method. Fans Nos. 1,2 and 5 work at a blade angle between No. 3 and No. 4 and speed of $718 \mathrm{rpm}, 715 \mathrm{rpm}$ and $715 \mathrm{rpm}$ as shown in Figures 5.11, 5.12 and 5.15, respectively. Fan No. 3 runs at a speed of $715 \mathrm{rpm}$ at a blade angle between 7B-7S and 2B-1S (Figure 5.13). Fan No. 4 (Figure 5.14) works at $715 \mathrm{rpm}$ too but at a blade angle No. 3. All subsystem characteristic curves look like a mine characteristic curve in a single fan system. They all pass through the origin and do not have a zigzag shape. Table 5.3 shows the measured and calculated operating points. The difference between the measured and the calculated values of the operating points is small. 
Table 5.3 Main mine fan's quantities and total pressures for mine C

\begin{tabular}{|c|c|c|c||c|c|c|}
\hline Fan \# & $\begin{array}{c}\text { Measured } \\
\text { Air } \\
\text { Quantity, } \\
\mathbf{m}^{\mathbf{3}} / \mathbf{s e c}\end{array}$ & $\begin{array}{c}\text { Calculated } \\
\text { Air } \\
\text { Quantity, } \\
\mathbf{m}^{\mathbf{3}} / \mathbf{s e c}\end{array}$ & Difference & $\begin{array}{c}\text { Measured } \\
\text { Pressure, } \\
\mathbf{P a}\end{array}$ & $\begin{array}{c}\text { Calculated } \\
\text { Pressure, } \\
\mathbf{P a}\end{array}$ & Difference \\
\hline 1 & 278.86 & 270.00 & 8.86 & $1,682.35$ & $1,775.14$ & -92.79 \\
\hline 2 & 252.47 & 252.00 & 0.47 & $1,758.57$ & $1,753.97$ & 4.60 \\
\hline 3 & 237.42 & 252.00 & -14.58 & $1,771.02$ & $1,668.57$ & 102.45 \\
\hline 4 & 213.00 & 203.00 & 10.00 & $2,137.18$ & $2,207.08$ & -69.90 \\
\hline 5 & 250.44 & 248.00 & 2.44 & $1,992.71$ & $2,061.52$ & -68.81 \\
\hline
\end{tabular}




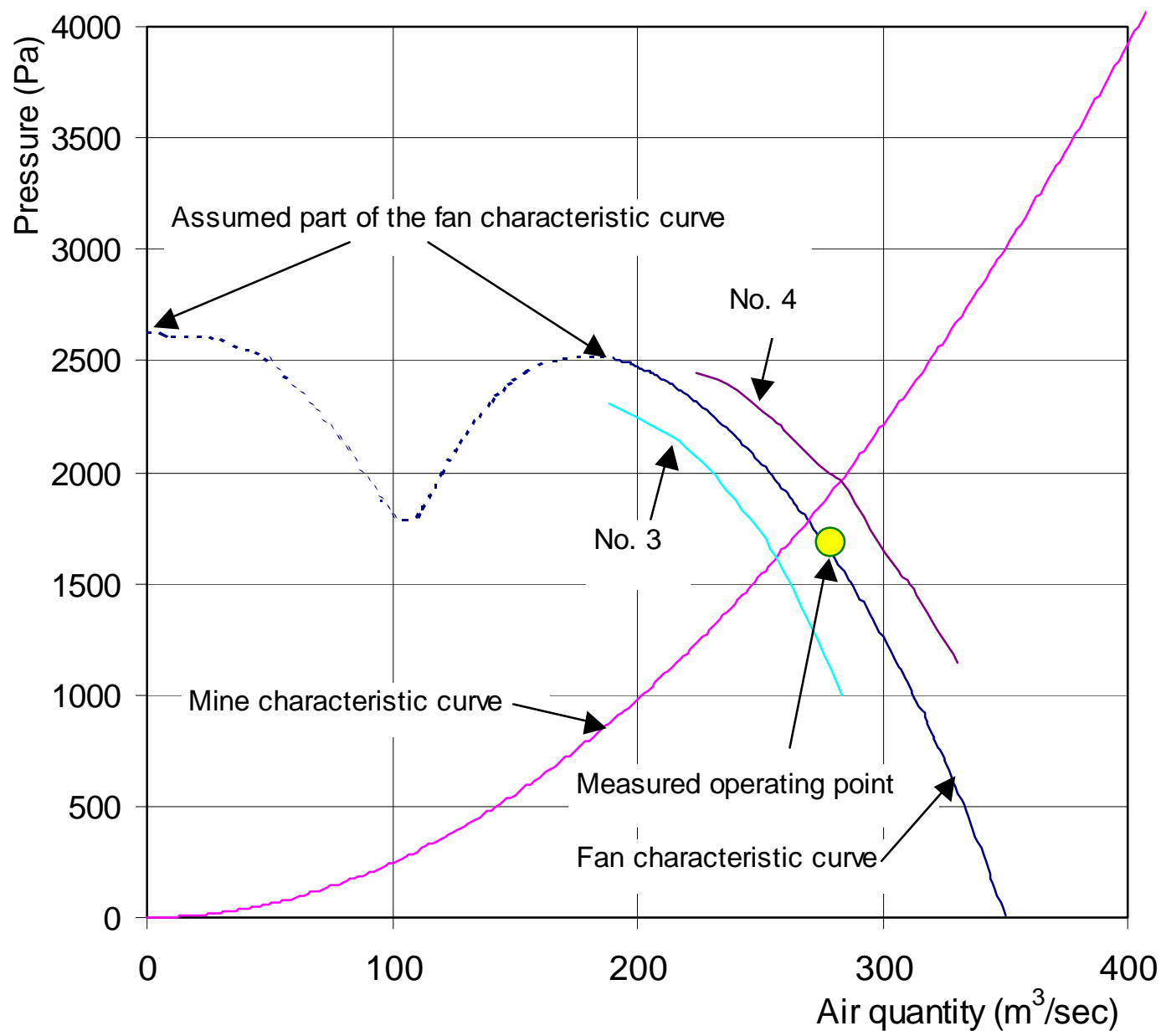

Figure 5.11 - Subsystem No. 1 characteristic curve (Mine C) 


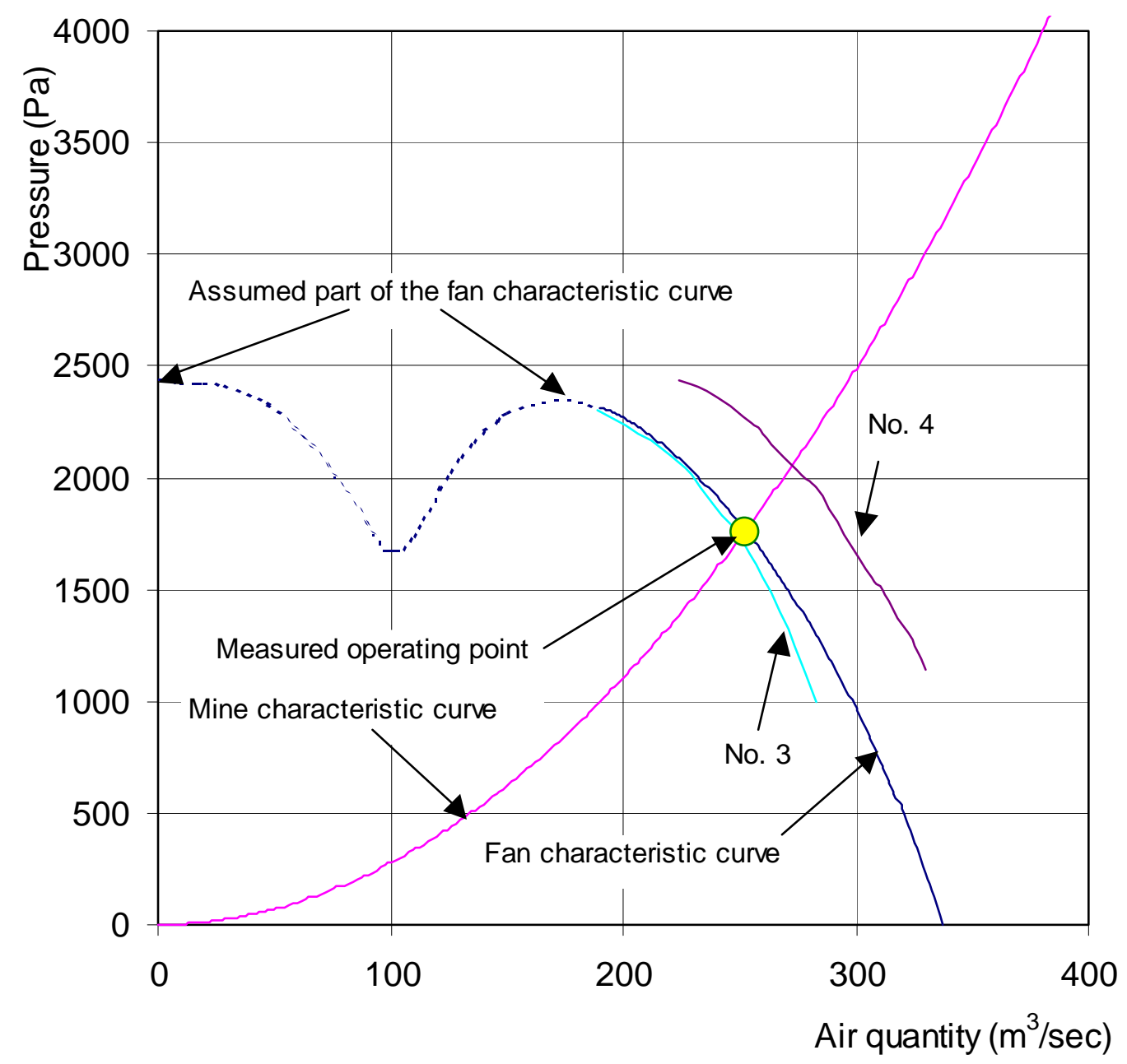

Figure 5.12 - Subsystem No. 2 characteristic curve (Mine C) 


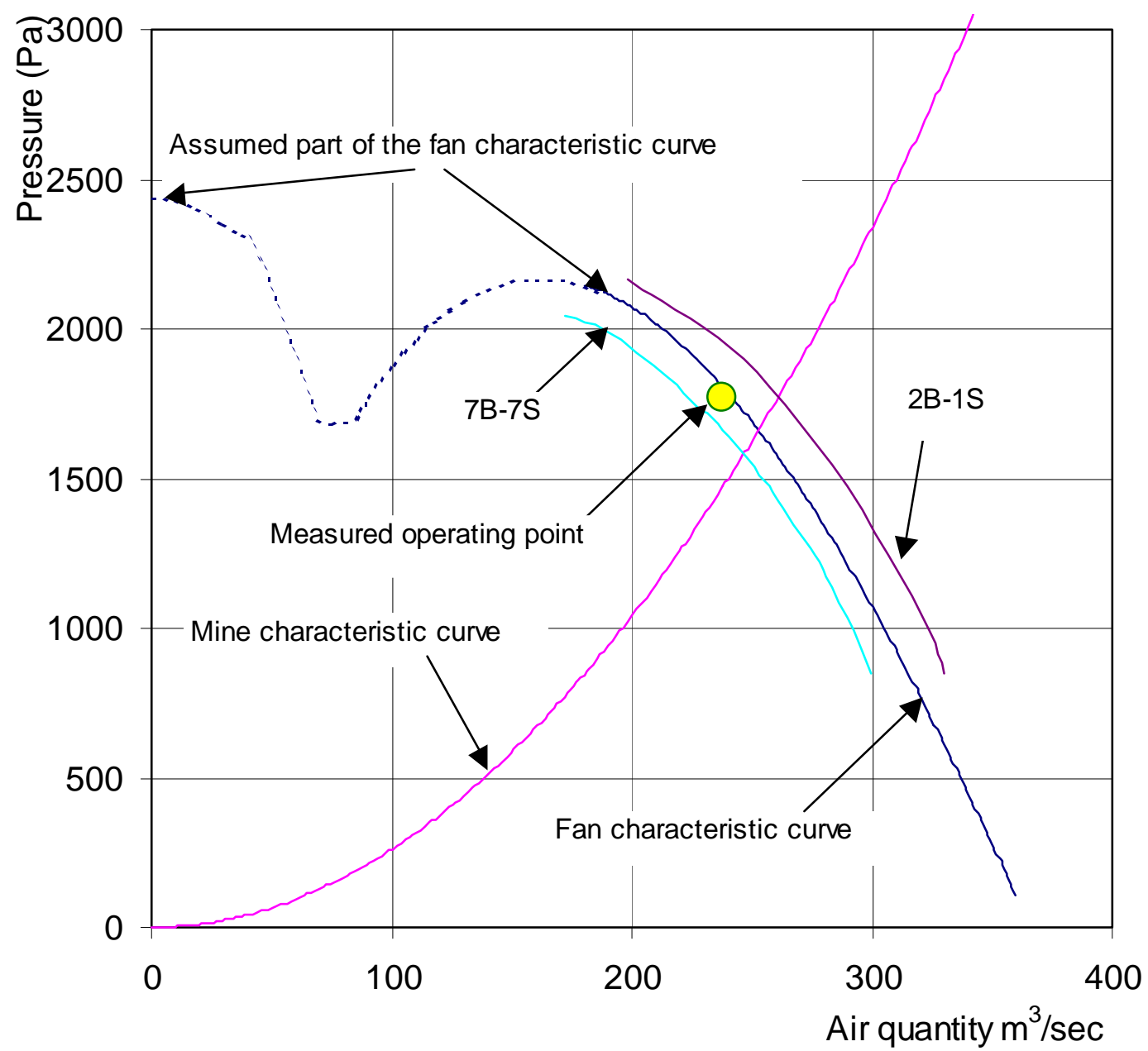

Figure 5.13 - Subsystem No. 3 characteristic curve (Mine C) 


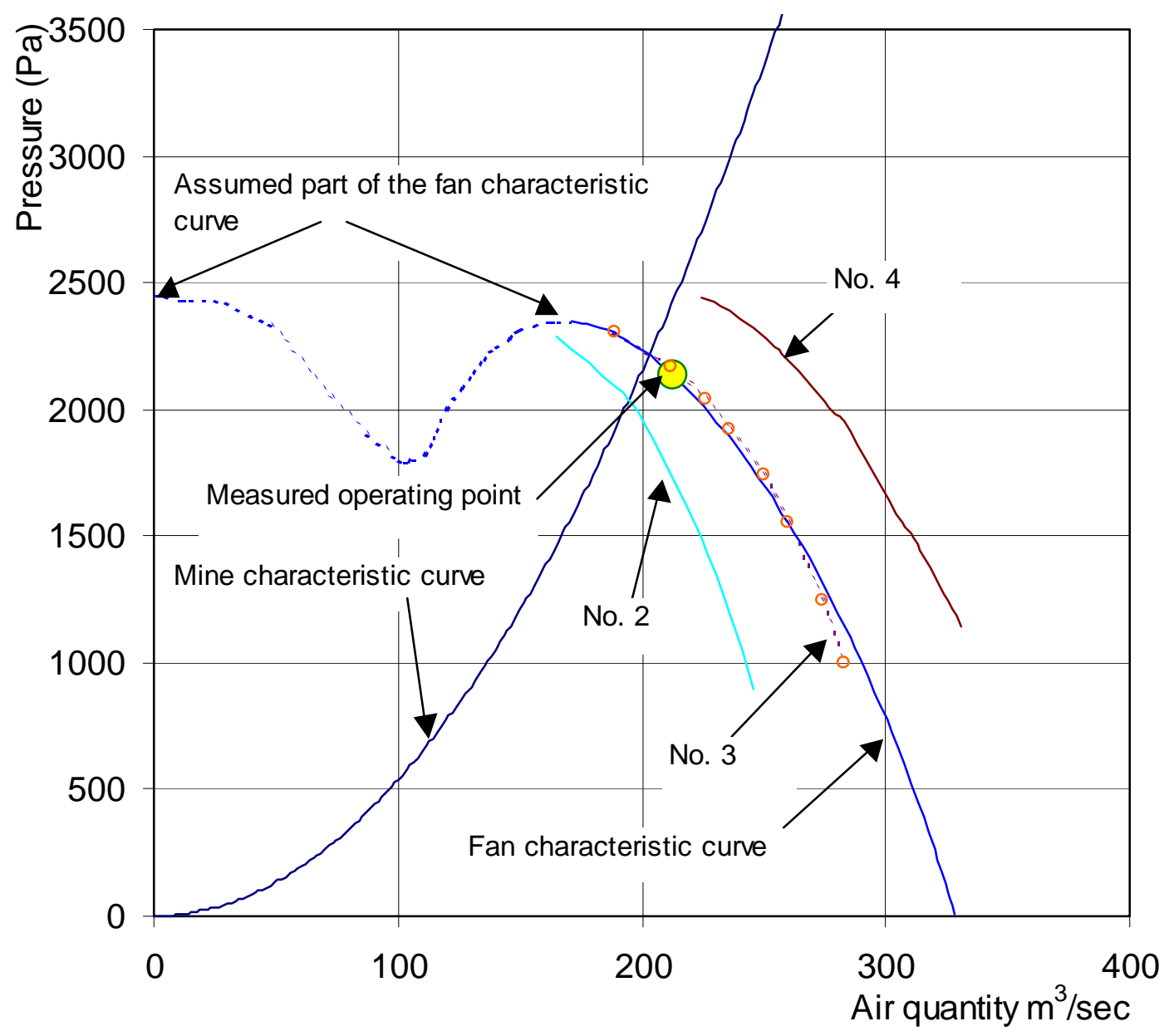

Figure 5.14 - Subsystem No. 4 characteristic curve (Mine C) 


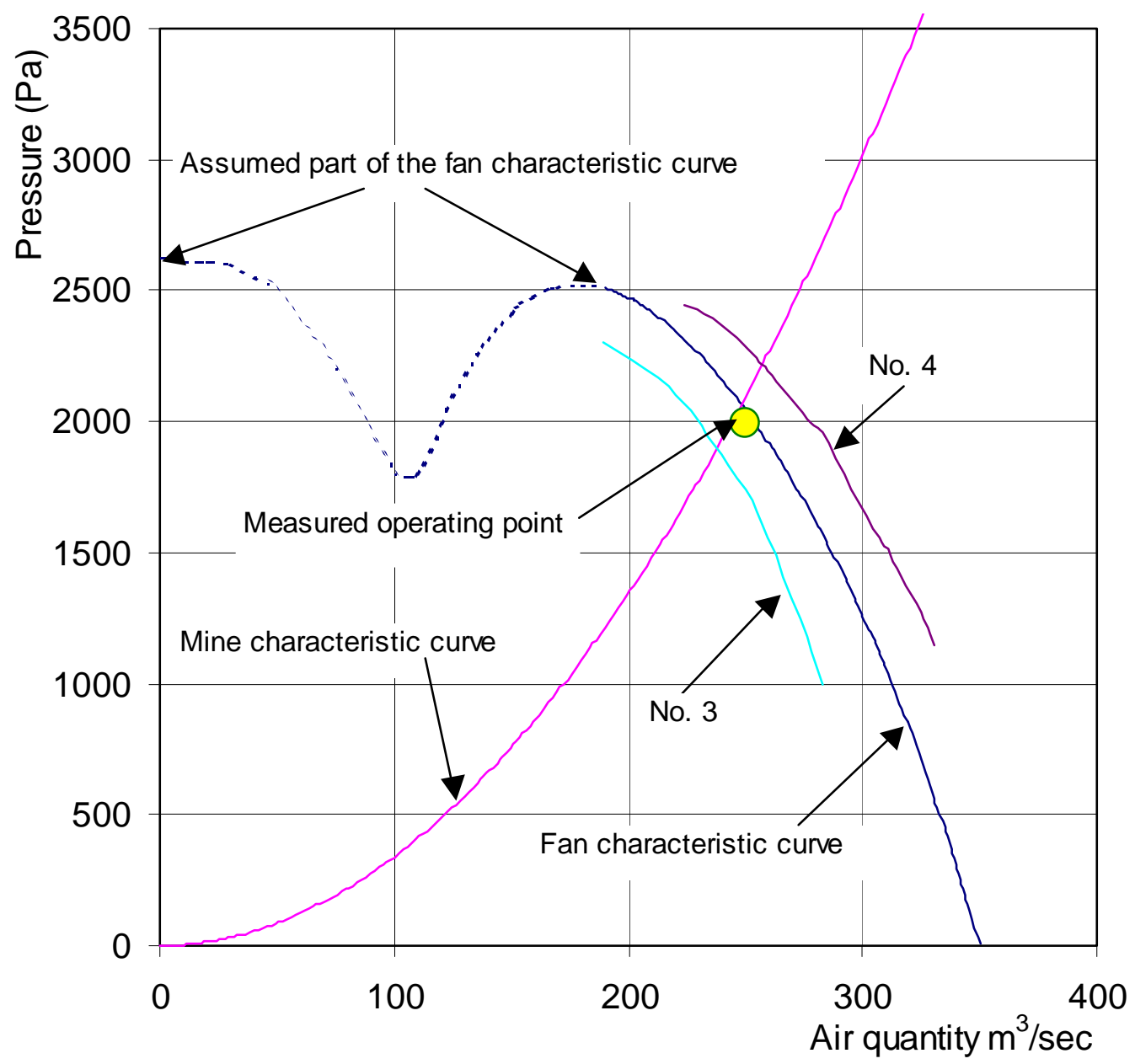

Figure 5.15 - Subsystem No. 5 characteristic curve (Mine C) 


\subsection{Relation Between Airflow and Resistance Factor in Real Coal Mine Networks}

In mine ventilation, the pressure loss across an airway of a single fan system is expressed as a function of air quantity, while the resistance is treated as a constant. The relation between air quantity and pressure, in a single fan system, is a strictly increasing function that can be drawn by applying Atkinson equation (Equation 2.3). For multiple fan systems, the resistance for any subsystem, and for the total system, is reliant upon the quantity ratios among the subsystems (Wang, 1984).

Figures 5.16 to 5.18 show the relation between the air quantity and the resistance factor for each subsystem in mines A, B and C, respectively. The effect of the fans inside any subsystem on the subsystem resistance is varied. This effect is considerable at small air quantities that flow across the subsystem. Referring to Figures 5.5 to 5.7, for mine A, if the subsystem characteristic curves do not pass through the origin then the resistances are very big at zero air quantity, Figure 5.16. For the subsystems of mines B and C, which pass through the origin, the resistances are less than $1 \mathrm{~N} \cdot \mathrm{s}^{2} / \mathrm{m}^{8}$ at zero air quantity (Figures 5.17 and 5.18).

All the fans inside a subsystem work as extra resistance. The effect is considerable at a small air quantity. The resistances are very big at zero airflow, then it may fall until it reaches a constant value, excepting the resistance of the subsystem No. 4 in mine $\mathrm{C}$, where the resistance starts small and then increases (Figure 5.18). Thus if a fan stops, the air flow direction in that branch would reverse, excluding the branch of fan No. 4 in mine C. In the subsystem No. 4 in mine C, all the fans assist it to exhaust the air 
from the mine and no airflow direction change is expected. Table 5.4 shows the values of constant resistance factor of each subsystem calculated after airflow of $100 \mathrm{~m}^{3} / \mathrm{sec}$.

Table 5.4 -Resistance for the subsystems

\begin{tabular}{|c|c|c|}
\hline Mine & Subsystem & Resistance, $\mathrm{N} . \mathrm{s}^{2} / \mathrm{m}^{8}$ \\
\hline \multirow{4}{*}{ A } & 1 & 0.0685 \\
\cline { 2 - 3 } & 2 & 0.0446 \\
\cline { 2 - 3 } & 3 & 00763 \\
\hline \multirow{4}{*}{ B } & 1 & 0.0288 \\
\cline { 2 - 3 } & 2 & 0.0678 \\
\cline { 2 - 3 } & 3 & 0.0475 \\
\cline { 2 - 3 } & 4 & 0.4642 \\
\hline \multirow{4}{*}{ C } & 1 & 0.0245 \\
\cline { 2 - 3 } & 2 & 0.0276 \\
\cline { 2 - 3 } & 3 & 0.0261 \\
\cline { 2 - 3 } & 4 & 0.0537 \\
\cline { 2 - 3 } & 5 & 0.0334 \\
\hline
\end{tabular}




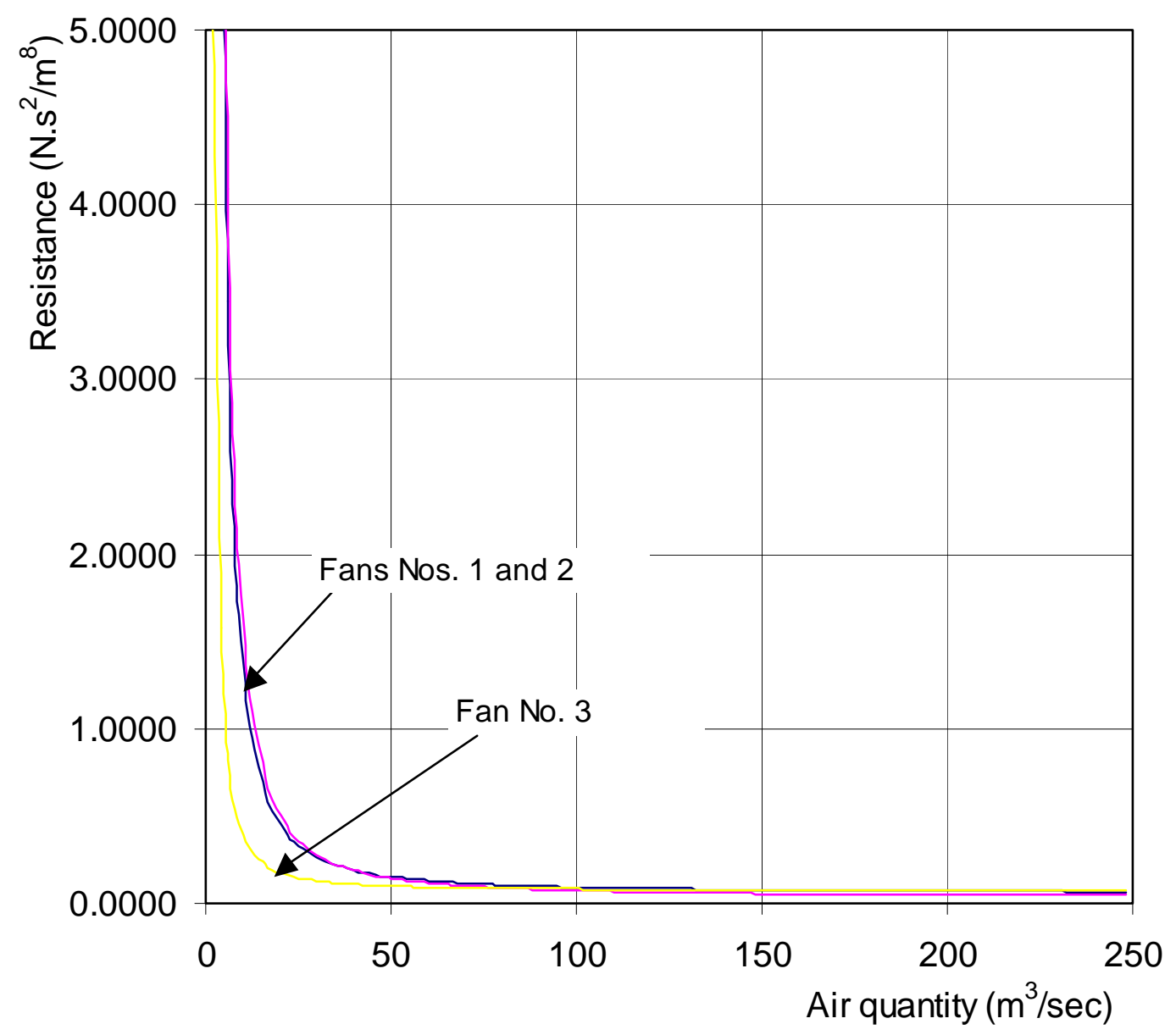

Figure 5.16 - The relation between air quantity and resistance factor in mine A 


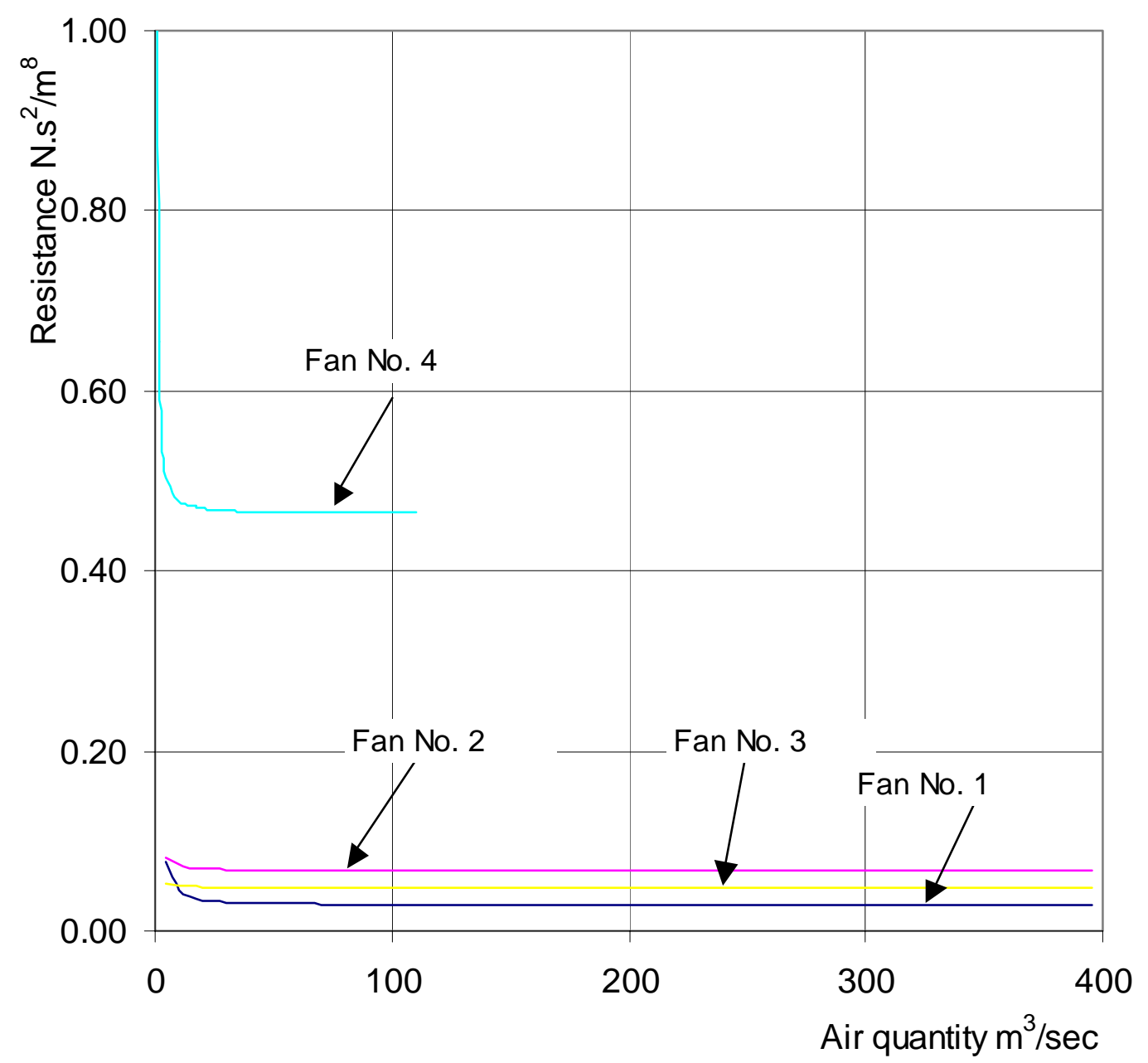

Figure 5.17 - The relation between air quantity and resistance factor in mine $B$ 


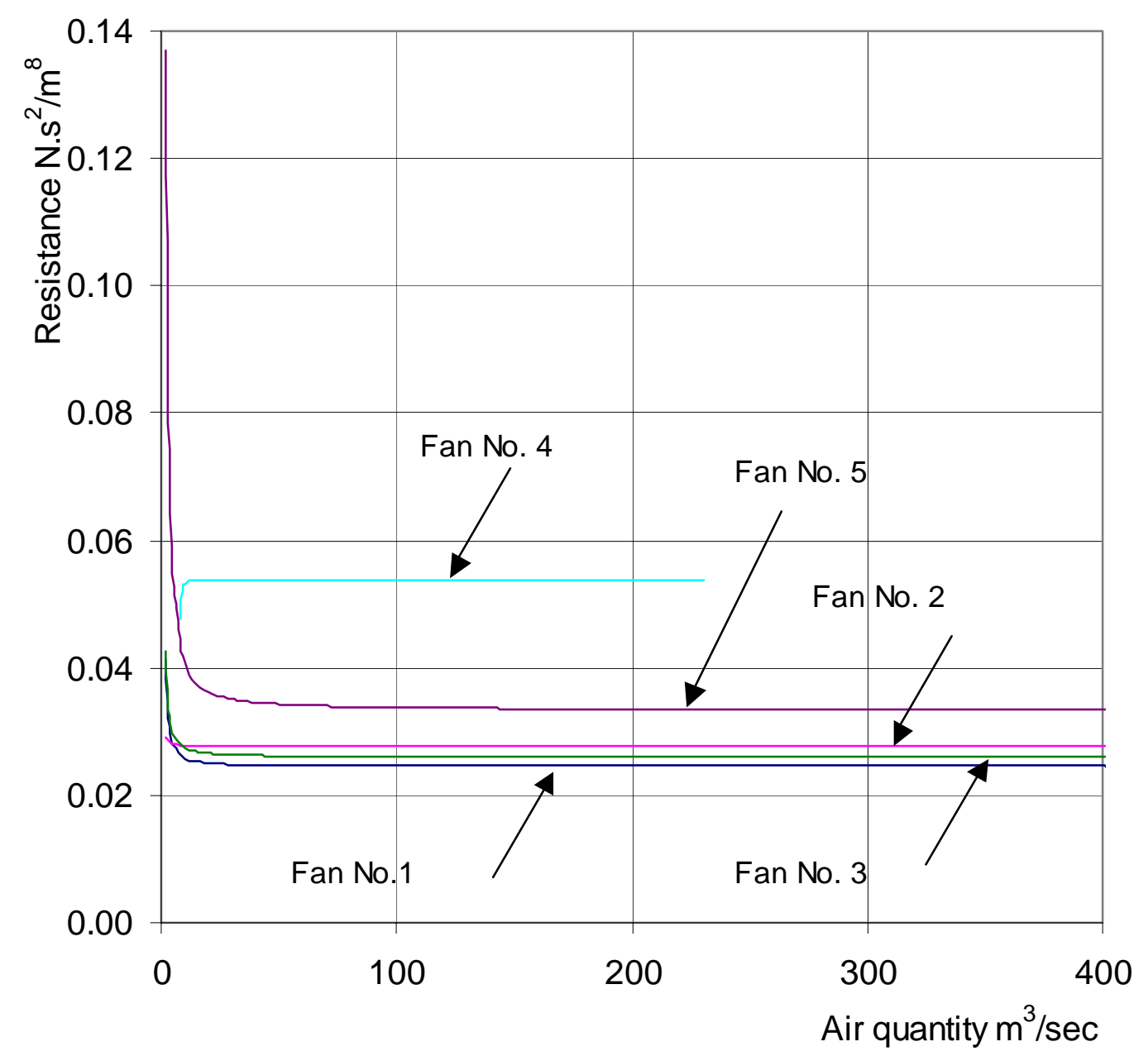

Figure 5.18 - The relation between air quantity and resistance factor in mine $\mathrm{C}$ 


\subsection{Summary}

Three different coal mines that applied multiple fan ventilation systems have been studied. The networks of mines $\mathrm{A}$ and $\mathrm{B}$ are too large to be solved by applying the modified damped Newton method. The Jacobian matrix and Gauss elimination technique need a very long time to calculate a new estimate of the solution. The modified Hardy Cross method has more capability in handling giant networks than the damped Newton method does.

The distance between fans in real multiple fan mines are far enough to vanish the bad mutual effect between them. Most fans work within the stable part of their characteristic curve. The shape of all the subsystem characteristic curves of the 12 fans in the three investigated mines do not have any turns and all are strictly increasing functions. There is some effect among the fans appearing in mine A, the subsystem characteristic curves for fans No. 1 and 2 do not pass through the origin. This effect is a helpful outcome and it would not effect on the stability of the system.

The measured and the calculated operating points are a good match in mines A and $\mathrm{C}$. The difference between the measured and the calculated operating point values in mine $\mathrm{B}$ are not small like the other two mines. The approximation of the unstable part of the axial flow fan, on the left of the stall point, has a large effect on the location of the operating point. 
The resistance factor for a subsystem may start big at a small airflow, but it reaches the real subsystem resistance value at a high airflows. This effect depends on the distance between the fans. The resistance factor of each subsystem, in well-designed coal mines, can be treated as a constant as in the single fan system. 


\section{CHAPTER 6 \\ CONCLUSION AND CONTRIBUTIONS}

\subsection{Conclusion}

In a single fan network, Atkinson's equation defined the relation between pressure consumed and airflow through the system. The graph of this relation represents the mine characteristic curve. Mine characteristic curve is a strictly increasing function, where mine resistance is treated as a constant value. The intersection between fan characteristic and mine characteristic curve is the operating point of the system and it can be obtained directly from the graph. In a single fan system, just one operating point is expected and so the multiple operating points can be easily avoided by changing fan setting angle, fan diameter and/or fan speed.

In a multiple fan network, each fan in the system has its own mine characteristic curve or subsystem characteristic curve. The subsystem resistance of each of those fans becomes a function not only of the network geometry but also the location and operating characteristic of other fans in the system. Graphical solution is applicable only to the simple series-parallel networks. Applying the principle of mine characteristic curve to the multiple-fan system using the subsystem approach can represent complex multiple-fan networks. Each subsystem has its own solution curve. Instead of the single mine characteristic in a single fan system, there is a subsystem characteristic curve for each fan in the system. The shape of subsystem characteristic curve in a multiple fan system does not have a fixed shape and does not follow Atkinson's equation. The number of equations defining any subsystem is one less the number of unknown parameters in the 
system. The solution curves for the subsystems are represented in a dimensional space equal to the unknown parameters in the system. This space is difficult to visualize if the number of unknowns in the network is greater than two.

However, even though the mathematical models that solve nonlinear equations can solve the single fan systems, they cannot be directly applied to multiple fan networks. To be able to trace the subsystem characteristic curves, the switching parameters algorithm has been employed to implement two famous mathematical models: Hardy Cross and Newton-Raphson. There is no fixed independent parameter in the switching parameters algorithm as it has been chosen at each iteration step. At each step, the independent parameters in multiple fan system algorithms have been chosen to be the airflow in a chord that has the largest change rate in the previous step.

While the modified Newton-Raphson algorithm cannot trace a complicated subsystem characteristic curve that has sharp turning points, the implemented damped Newton-Raphson method seems to be a good tool for solving multiple fan systems. Compared to the damped Newton-Raphson method, the Hardy Cross method has a higher convergence rate and has shown more capability in handling big networks.

Multiple operating points in multiple fan systems are due to two main factors. The first factor is the fan operating zone and the second is the shape of the subsystem characteristic curve. Improving the network parameters like resistance factors, fan speed, fan type, fans number and topology of the network can control both factors. Increasing 
the fan branch's resistance in multiple fan systems increases the slope of its subsystem curve, subsequently decreasing the slope of the other fan's subsystems in the network. A regular branch has the same effect as single fan systems on all the subsystems, decreasing the regular branch resistance, reducing the subsystem pressure. The effect of changing the topology of the network is considerable and varied among the subsystems in the network. The shape of the subsystem characteristic curves moves toward the common mine characteristic curve of a single fan system while decreasing the over all resistance of the network. Decreasing the subsystem resistance can be done by increasing the number of branches, connections and so the number of meshes in the network. Consequently the slope of the subsystem characteristic curve decreases, which leads to decreasing the number of operating points. Fans inside any subsystem work as resistances. Increasing the number of axial flow fans in the system increasing the subsystem resistance and increasing the sharp turning points in the subsystem characteristic curves. The speed of fans inside any subsystem has the same effect as changing the number of axial flow fans in the system, especially if increasing the speed of one fan leads to one or more of the fans work in its/their unstable zone. Finally, increasing the number of centrifugal fans in the system increases the stability of the subsystems.

In real multiple fan coal mines, the mutual effects between fans are feeble because they are usually installed very far from each other and most of them work within the stable zone of their characteristic curves. This effect may appear at a small air quantity delivered from any investigated fans as extra pressure recognized in the fan branch results from the effect of the other fans in the system. This extra pressure does not affect 
the stability of the system. The subsystem's resistance factor is not constant, it sizeable at a small airflow, then drops until it reaches a fixed value at high airflows.

\subsection{Contribution}

- The main contribution of this work is the simultaneous determination of all operating points in a multiple fan system with a single try.

- A thorough review has been conducted on both single and multiple fan systems. This review has explained the differences between the two systems and led to several conclusions that help researchers tackle the problem of multiple fan systems.

- Clarified the meaning of the subsystems and the subsystem equations.

- The applicability of the graphical solution to the multiple fan system has been clarified.

- The applicability of the modified Hardy Cross and the modified Newton Raphson method to the multiple fan system and the superiority of Hardy Cross over the damped Newton Raphson to handle big networks.

- The switching parameters algorithm and it is application in multiple fan system has been elucidated.

- The shape of the subsystem characteristic curve and the relation between this shape and the number of operating points in the system.

- The relation between the shape of the subsystem characteristic curve and the resistance factor, fan number, fan speed, fan type and the topology of the network has been investigated. 
- The shape of the subsystem characteristic curves in real mines has been inspected.

- The difference between the system resistance in the single fan system and multiple fan system has been defined. 


\section{References}

Barnes, E. L. S., 1976, "Mine Ventilation Network Analysis," Proceedings of the International Mine Ventilation Congress, Mine Ventilation Society of South Africa, Johannesburg, pp. 5-8.

Bernardo, P. M., and Gama, C. D., 1997, " A Methodology for Design and Simulation of Mine Ventilation Networks," Proceedings of the $6^{\text {th }}$ International Mine Ventilation Congress, pp. 395-402.

Bhamidipati, S. S., and Procarione, J. A., 1985, "Linear Analysis for the Solution of Flow Distribution Problems," Proceedings of the $2^{\text {nd }}$ US Mine Ventilation Symposium, Mousset_Jones, P. (Ed.), Rotterdam, Netherlands, pp. 645-654.

Bunt, H. J., Metcalf, J. R., Pursall, B. R., Roberts, A., Williams, F. T., 1960, ” Mine Ventilation," $1^{\text {st }}$ edition, Roberts, A (Ed.), Cleaver-Hume Press LTD, London, pp 296327.

Burden, R.L., and Faires, J.D., 1985, "Numerical Analysis," $3^{\text {rd }}$ ed., Prindle, Weber \& Schmidt, Boston, pp. 117-122.

Chua, L. O., and Ushida, A., 1976, "A Switching-Parameter Algorithm for Finding Multiple Solutions of Nonlinear Resistive Circuits," International Journal of Circuit Theory and Applications, Vol. 4, No. 3, pp. 215-239.

Collins, M., Cooper, L., Helgason, R., Kennington, J., and LeBlanc, L., 1978, "Solving the Pipe Network Analysis Problem Using Optimization Techniques," Management Science, Vol. 24, pp. 747-760.

Conte S.D. and Carl de Boor, 1980, "Elementary Numerical Analysis An Algorithm Approach," McGraw-Hill Inc., $3^{\text {rd }}$ Ed. pp. 216-222.

Cross, H., 1936, " Analysis of Flow in Networks of Conduits or Conductors," Bulletin 286, Engineering Experiment Station, University of Illinois, Urbane, 29 pp.

English, L. E., and Wang, Y. J., 1998, "Characteristic Curves Revisited: A more Logical Approach to Determining Operating Points," Preprint No. 97-53, SME, Denver, CO., 4 pp.

Gregory, W. S., Nicholas, B. D., and Idzorek, R., 1987, "Node-Based Network Analysis and Its Application to Mine Ventilation," Proceedings of the $3^{\text {rd }}$ Mine Ventilation Symposium, SME, Littleton, Co., pp. 583-588. 
Harrison, S.P., and Kutay, V.S., 1986, “An Analysis of Mining Fan Irregularities Relative to Underground Conditions, Ventilation, and Potential Fan Defects," Proceedings of $2^{\text {nd }}$ Mine Ventilation Symposium, Mousset-Jones, P., Ed., Vol. 2, A.A. Balkema, Rotterdam, Netherlands, pp. 821-838.

Hartman, H. H., Mutmansky, J. M., Ramani, R. V., and Wang, Y. J., 1997, "Mine Ventilation and Air Conditioning," John Wiley \& Sons inc., NY., USA, $3^{\text {rd }}$ edition..

Hu, W., and Longson, I., 1990, "The Optimization of Airflow Distribution in Ventilation Networks Using A Nonlinear Programming Method," Mining Science and Technology, Vol. 10, No. 2, pp. 209-219.

Huang, C., Lin, S., and Wang, Y. J., 1993, "Constructing Subsystem Characteristic Curves for An Experimental Two-Fan Network by Laboratory Measurement," Trans. SME, Vol. 294, pp. 1858-63.

Jacques, E. J. P. M., 1976, "Generalization of Cross Iterative Method for Computing Ventilation Network," Proceedings of the International Mine Ventilation Congress, Mine Ventilation Society of South Africa, Johannesburg, pp. 1-3.

Jorgensen, R., 1983, "Fan Engineering," Buffalo Forge Company, Buffalo, NY., $8^{\text {th }}$ edition, 9-488.

Kamba, G. M., Jacques, E., and Patigny, J., 1995, “Application of the Simplex Method to the Optimal Adjustment of the Parameters of A Ventilation Network," Proceedingss of the $7^{\text {th }}$ US Mine Ventilation Symposium, Wala, A. M. (Ed.), SME, Littleton, Co., pp. 461-465.

Kim, J. H., and Mutmansky, J. M., 1992, "An Empirical Study of the Effects of Mesh Selection Procedures on Efficiency of Mine Ventilation Analysis Methods" Trans. SME, Vol. 292, pp. 1856-1860.

Kingery, D.S., and Kapsch, F.F., 1959, "Airflow Changes in Multiple-Fan Systems" Bureau of Mines, United States Department of the Interior, Information Circular 7889.

Kumar, G. V., Sastry, V. R., and Rao, G. K., 1995, "Minimizing Power Consumption in Multiple Fan Networks by Optimum Fan Selection," Proceedings of the $7^{\text {th }}$ US Mine Ventilation Symposium, SME, Littleton, Co., pp. 491-497.

Kumazawa, Y., 1942, "The Triangular Transformation of Ventilation of Networks," Journal of Mining Institute of Hokkaido, Vol. 1, No. 5, pp. 203-207.

Lin, S., 1995, "Operating Characteristics and Stability of Multiple-Fan Ventilation System," Doctoral Dissertation, West Virginia University, Morgantown, WV. 
Lin, S., and Wang, Y. J., 1993(a), "Determining Operating points of Multiple-Fan Ventilation Systems by Tracing An Augmented-System Solution Curve," Trans. SME, Vol. 294, pp. 1845-1850.

Lin, S., and Wang, Y. J., 1993(b), "Understanding Subsystem Characteristic Curves and Operating Points of Multiple-Fan Ventilation System," Proceedings of the $6^{\text {th }}$ US Mine Ventilation Symposium, Bhashar, R. (Ed.), SME, Littleton, Co., pp. 231-236.

Mcllroy, G. T. R., 1987, "Problem with Ventilation Network Analysis," Transaction of the Institution of Mining and Metallurgy, Section A, Mining Industry, Vol. 96, pp.A37A39.

McPherson, J. M., 1993, "Subsurface Ventilation and Environmental Engineering," Chapman \& Hall, London.

Mishra, R., Ramani, R. V., and Wang, Y. J., 1978, "System Concept for Coal Mine Ventilation,” Mining Engineering, Vol. 30, No. 11, pp. 1569-1175.

Morris, I. H., and Hinsley, F. B., 1951, "Some Factors Affecting the Choice of Fans for Mine Ventilation,” Trans. Inst. Mng. Engr., (London), Vol. 111, pp. 489-524.

Mousset-Jones, P, 2001, Personal Recommendation.

Mutmansky, J. M., and Kim, J., H., 1993, " A Study of Mesh Ordering Techniques for Mine Ventilation Network Solution Methods," Proceedings of the $6^{\text {th }}$ US Mine Ventilation Symposium, Bhashar, R., Ed., SME, Littelton, Co., pp. 163-169.

Nash, G. S, and Sofer, A., 1996, "Linear and Nonlinear Programming," McGraw-Hill Companies, NY., pp. 37-45.

Ortega, J. M., and Rheinboldt, W. C., 1970, "Iterative Solution of Nonlinear Equations in Several Variables," Academic Press, NY.

Pompei, M.A., Schroeder, M.E., Weaver, D.L., and Stoltz, R.T., 1998, " Mine Ventilation Air Pressure/Quantity Investigation," Investigation Report No. P418-V323, Consolidation Coal Company, Rend Lake Mine I.D. No. 11-00601, Sesser, Jefferson County, Illinois.

Ramani, R. V., 2001, Personal Recommendation.

Schroeder, M.E., Weaver, D.L., Pompei, M.A., Gandy, B.W., Diederich, K.S., Stoltz, R.T., and Laage, L.W., 1998, " Mine Ventilation Air Pressure/Quantity Investigation," Investigation Report No. P411-V316, Eighty-Four Mining Company, Mine 84, I.D. No. 36-00958, Eighty Four, Washington County Pennsylvania. 
Scott, D. R., and Hinsley, F. B., 1951, "Ventilation Network Theory," Colliery Engineering, London, Vol. 28, No. 324, 326, 328, and 334, pp. 67-71, 159-166, 229235 , and 497-500.

Tominaga, Y., Matsukura, H., and Higuchi, K., 1985, “Algorithm for Fast Simulation of Mine Ventilation Using Dual Microcomputer," Proceedings of the $2^{\text {nd }}$ US Mine Ventilation Symposium, Mousset_Jones, P. (Ed.), Rotterdam, Netherlands, pp. 499-504.

Ueng, T. H., and Wang, Y. J., 1984, "Analysis of Mine Ventilation Networks Using Nonlinear Programming Techniques," International Journal of Mining Engineering, Vol. 2, No. 3, pp. 245-252.

Urosek, J.E., 1997, "Partial Pressure/Air Quantity Survey at the Oak Grove Mine” ID No. 01-00851, U.S. Department of Labor, Ventilation Division.

Vutukuri V. S., and Lama R. D., "Environmental Engineering in Mines," Cambridge University Press., 1986.

Wang, Y. J., 1982(a), " Ventilation Network Theory," Mine Ventilation and Air Conditioning," $2^{\text {nd }}$ ed., H. L. Hartman (Ed.), Wiley-Interscience, NY, pp. 167-195.

Wang, Y. J., 1982(b), "Critical Path Approach to Mine Ventilation Networks with Controlled Flow," Trans. SME-AIME,Vol. 272, pp. 1862-72.

Wang, Y. J., 1983, "Minimizing Power Consumption in Multiple-Fan Network by Equalizing Fan Pressure," International Journal of Rock Mechanics and Mining Science, Vol. 20, No. 4, pp. 171-179.

Wang, Y. J., 1984(a), "A Non-Linear Programming Formulation for Mine Ventilation Networks with Natural Splitting," International Journal of Rock Mechanics and Mining Science, Vol. 21, No. 1, pp. 42-3-45.

Wang, Y. J., 1984(b), "Characteristic of Multiple-Fan Ventilation Networks," International Journal of Mining Engineering, Vol. 2, pp. 229-243.

Wang, Y. J., 1985, "Graphical Representation of the Operating Points for Two-Fan Ventilation Systems," Trans. SME-AIME, Vol. 278, pp. 1888-92.

Wang, Y. J., 1988, "A Coding Scheme for A Graphical Solution to Mine Ventilation Networks," Mining Science and Technology, Vol. 7, No. 1, pp. 31-43.

Wang, Y. J., 1989, "A Procedure for Solving A More Generalized System of Mine Ventilation Network Equations," Proceedings of the $4^{\text {th }}$ US. Mine Ventilation Symposium, SME, Littleton, Co., pp. 419-424. 
Wang, Y. J., 1990, "Solving Mine Ventilation Networks with Fixed and Non-Fixed Branches," Mining Engineering, Vol. 42, No. 12, pp. 1342-1346.

Wang, Y. J., 1992, "Characteristic Curves for Multiple-Fan Ventilation Systems," Trans. SME, Vol. 292, pp. 1829-1836.

Wang, Y. J., 1993, "Characteristic Curves for the Series-Parallel Ventilation Network with Multiple Fans," Trans. SME, Vol. 294, pp. 1821-1827.

Wang, Y. J., 1997, " An Algorithm for the Exact Solution of A Mesh Equation in the Hardy Cross Iterative Method for Ventilation Network analysis," Trans. SME, Vol. 302, pp. $145-150$.

Wang, Y. J., and Hartman, H. L., 1967, "Computer Solution of Three-Dimensional Mine Ventilation Networks with Multiple Fans and Natural Ventilation," Int. J. Rock Mech. Mng. Sci., Oxford, Vol. 4, pp. 129-154.

Wang, Y. J., and Saperstein, L. W., 1970, "Computer-Aided Solution of Complex Ventilation Networks," Trans. SME-AIME, Vol. 247, pp. 238-250.

Wang, Y. J., Hartman, H. L., and Mutmansky, J. M., 1985, "Recent Developments in Mine Ventilation Network Theory and Analysis," Proceedings of the $2^{\text {nd }}$ Mine Ventilation Symposium, Balkema, A. A. (Ed.), Rotterdam, Netherlands, pp. 667-675.

Wang, Y. J., and Ready, N. P., 1992, "Fan Stall and Multiple Points in Multiple-Fan Ventilation Systems," Mining engineering, Vol. 44, No. 2, pp. 167-172.

Wang, Y. J., and Tominaga, Y., 1996, "Delta-Y Reduction for Ventilation Networks," Proceedings of the 96 International Symposium on Mining Science and Technology, Guo \& Golosinski (eds.), Balkema, Rotterdam.

Willis, R. A., 1961, “Axial Flow Fans, Design and Practice, " $1^{\text {st }}$ Ed., Academic Press Inc., New York, pp 260-275. 


\section{APPENDIX B LIST OF COMPUTER CODES USED IN THIS STUDY}

\section{B.1 Introduction}

This appendix contains list of the codes used in this study to conduct different tasks as well as comments and directions on how to use these files. Both files hc.m and Newton.m draw the solution curve for any subsystem.

The file hc.m, appendix B.2, depends mainly on the famous Hardy Cross method to calculate the airflow through the branches. This algorithm calls functions mesh.m, resis.m, t1.m, t2.m, ind.m, eval_f.m, try1.m, try2.m and try3.m. The function mesh.m, appendix B.2.1, contains the fundamental mesh matrix for the illustrative example shown in Figure 3.4. Appendix B.2.2 shows function resis.m that contains the resistance values of each branch in the network in the illustrative example in $\mathrm{N} . \mathrm{s}^{2} / \mathrm{m}^{8}$. The function ind.m, appendix B.2.3, compares between the values of the old and the new estimates of the airflows and pick the branch that shows the largest change in the airflow among them. Function eval_f.m, appendix B.2.4, calculate the value of the functions, Equations 2.6 to 2.8. The functions $\mathrm{t} 1 . \mathrm{m}$ and $\mathrm{t} 2 . \mathrm{m}$, appendices A.2.5 and A.2.6 respectively, contain the data for the axial flow fans type (A) and (B) respectively, Figure 3.4. The functions try1.m, try2.m and try3.m, appendices A.2.7, A.2.8 and A.2.9 respectively calculate the new values of the airflow through each branch of the network at each step of the 
algorithm at three different cases. First case, when the maximum airflow change occurs at the same direction as the subsystem that under investigation. Second case, when the maximum airflow change takes place in the direction clash with the subsystem under investigation but it coincides with one of the subsystems in the network. Finally, case three, when the maximum airflow change happens in the direction of any of the regular branches in the network.

The file Newton.m, appendix B.3.1, depends mainly on the damped Newton method to solve the mine ventilation networks. This file as hc.m calls functions mesh.m, resis.m, ind.m, eval_f.m, t1 and t2.m beside these functions it calls functions gauss.m and jacob.m. Appendix B.3.1 shows function guass.m that contain the computer code to eliminate the linear function and calculate the new estimate of the solution by using the guess elimination technique. Function jacon.m, Appendix B.3.2, calculates the Jacobian matrix of the nonlinear equations of the system.

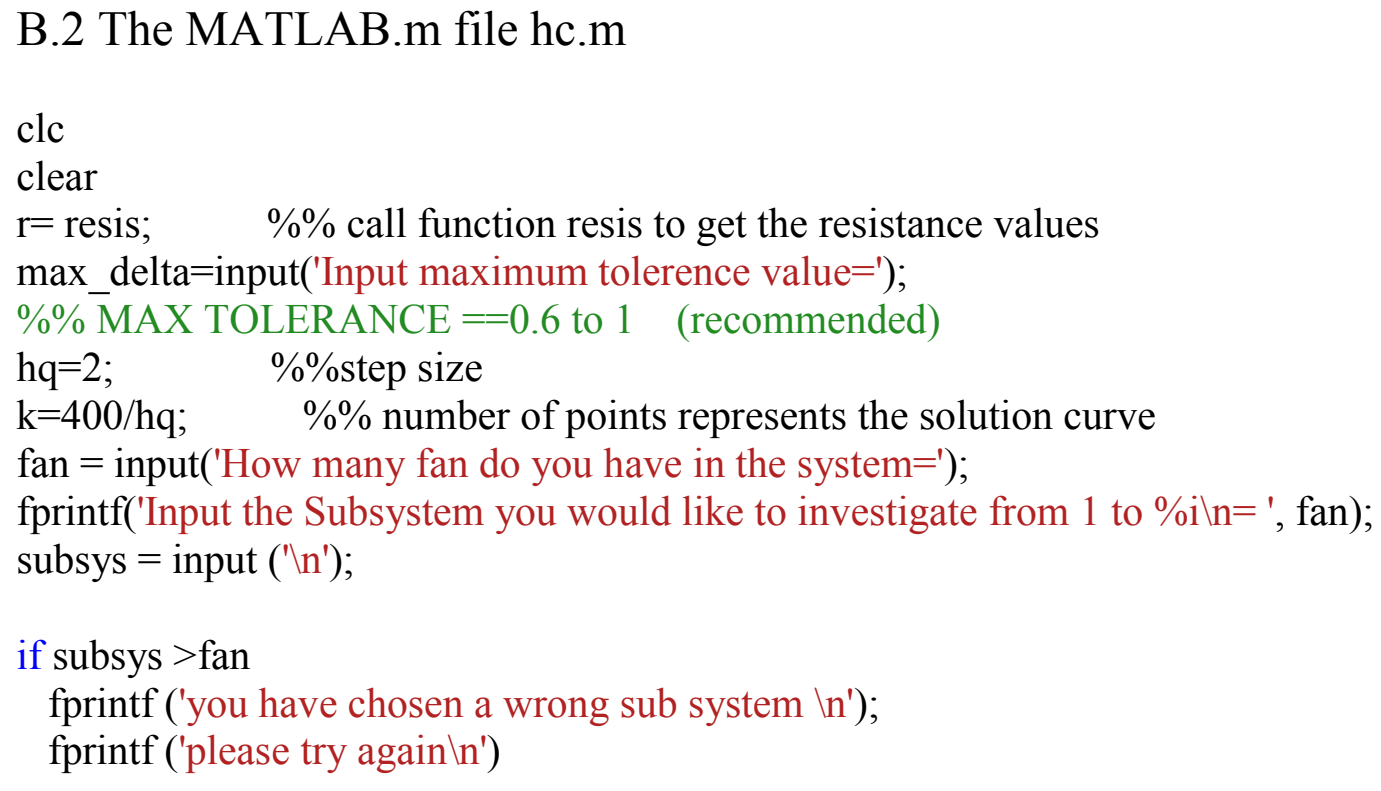




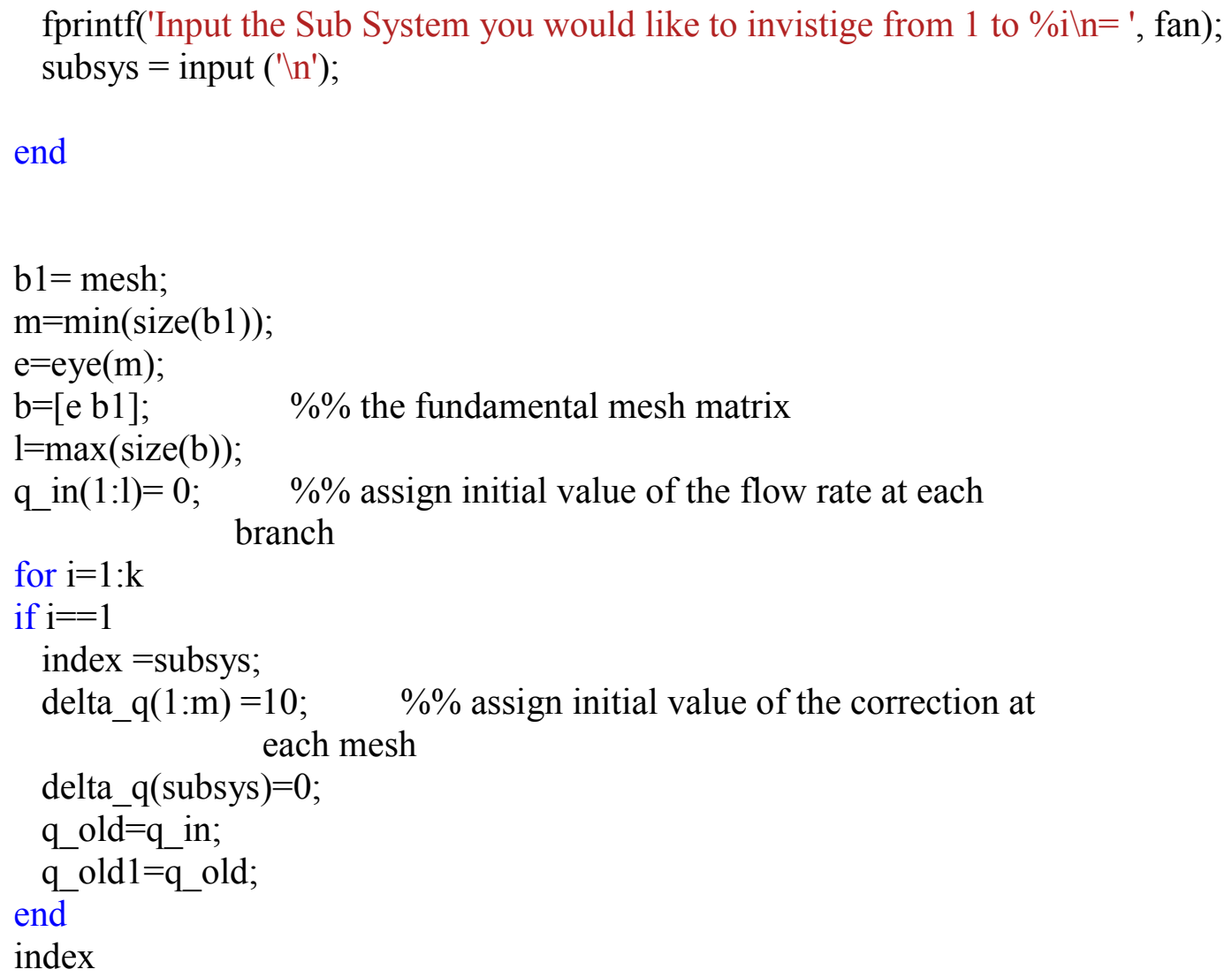




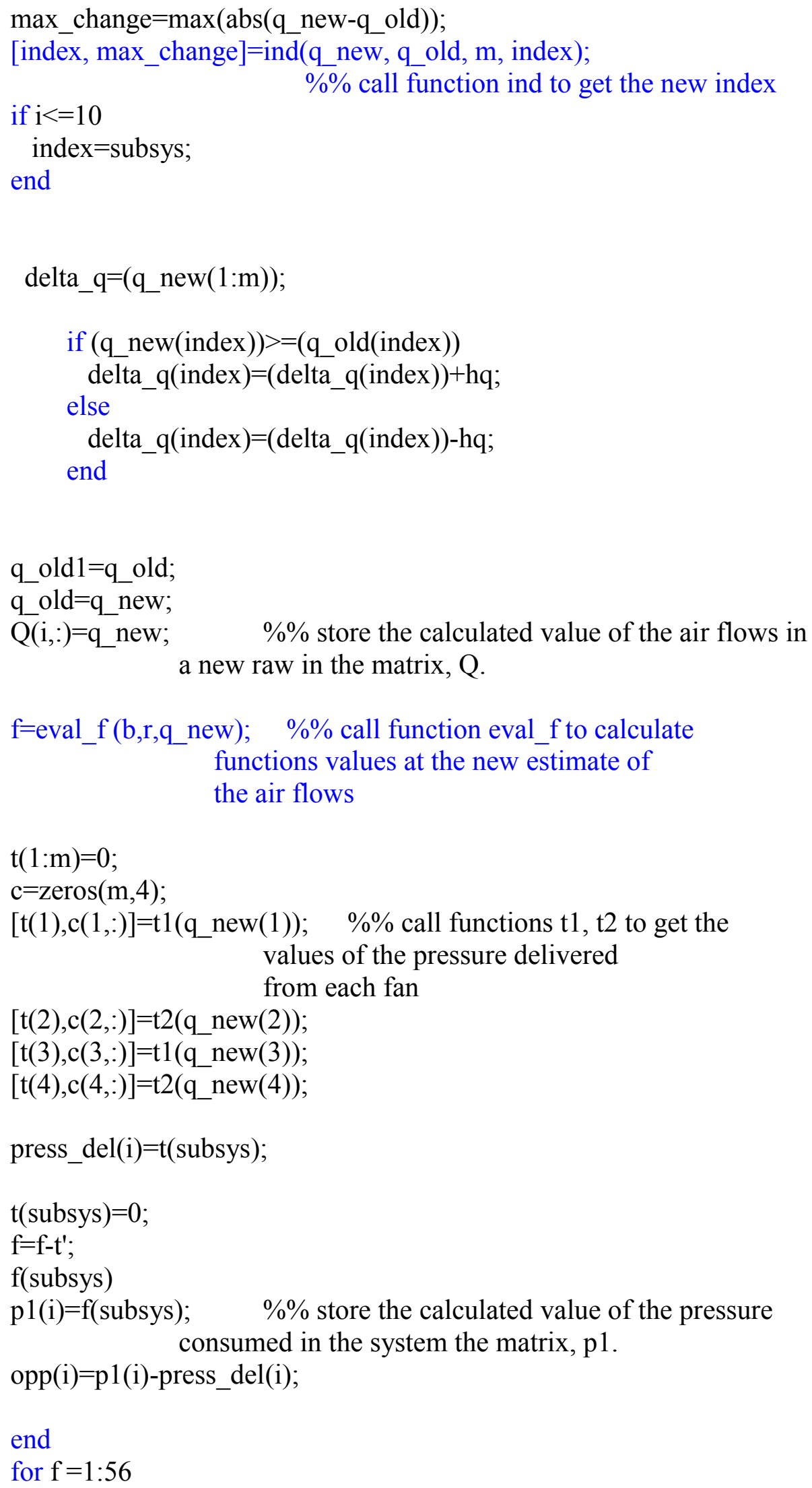




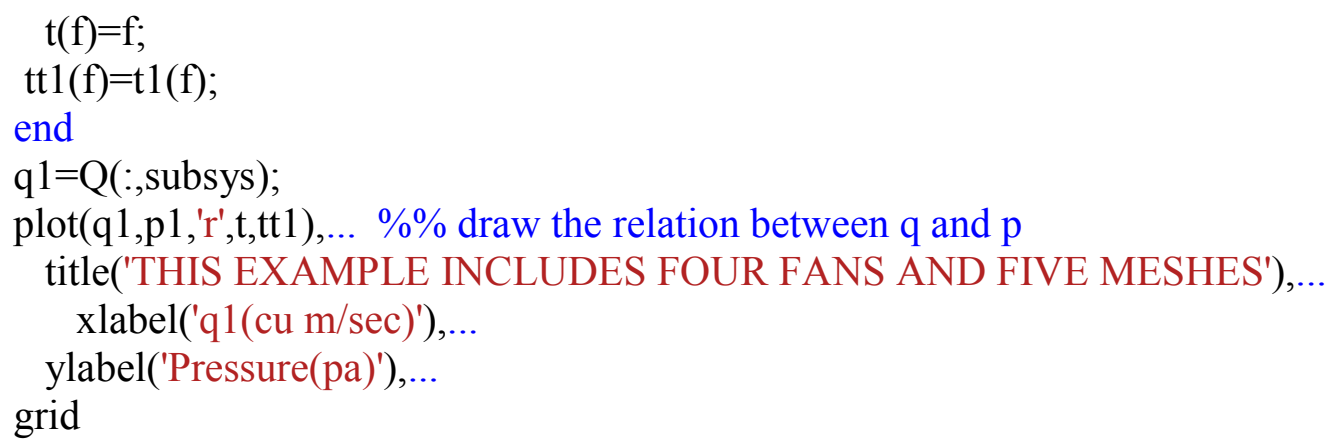

\section{B.2.1 The MATLAB.m file mesh.m}

$\% * * * * * * * * * * * * * * * * * * *$ fundamental mesh $* * * * * * * * * * * * * * * * * * * * * * * * * *$

$\% * \quad$ This function stores the Fundamental_Mesh Matrix matrix for the illustrative example that has four fans and five meshes

$\% * * * * * * * * * * * * * * * * * * * * * * * * * * * * * * * * * * * * * * * * * * * * * * * * * * * * * * * * * * * * * * * *$

$$
\begin{aligned}
& \text { function } \mathrm{b} 1=\operatorname{mesh}() \text {; } \\
& \text { b1 }=\left[\begin{array}{lllll}
0 & 0 & 1 & 0 & 1
\end{array}\right. \\
& \begin{array}{llllll}
-1 & -1 & 0 & 1 & 1
\end{array} \\
& 0-10 \begin{array}{llllll}
-1 & 0 & 1 & 1
\end{array} \\
& \begin{array}{lllllllllll}
0 & 0 & 0 & 1 & 1
\end{array} \\
& \left.\begin{array}{lllll}
1 & 1 & 1 & -1 & 0
\end{array}\right] \text {; }
\end{aligned}
$$

\section{B.2.2 The MATLAB.m file resis.m}

$\% * * * * * * * * * * * * * * * * * * *$ resistance factor $* * * * * * * * * * * * * * * * * * * * * * * * * *$

$\% * \quad$ This function stores the resistance factor for the illustrative example that has four fans and five meshes

$0 \% * \quad 0 * * * * * * * * * * * * * * * * * * * * * * * * * * * * * * * * * * * * * * * * * * * * * * * * * * * * * * * * * * * * *$

function $\mathrm{r}=$ resis( $)$;

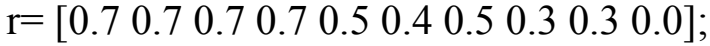

\section{B.2.3 The MATLAB.m file ind.m}

$\% * * * * * * * * * * * * * * * * * * * * * * * * * * * *$ ind. $\mathrm{m} * * * * * * * * * * * * * * * * * * * * * * * * * * * * * * *$

$\%$ This function to define the right path to follow according to the switching parameters algorithm

$0 \% * * * * * * * * * * * * * * * * * * * * * * * * * * * * * * * * * * * * * * * * * * * * * * * * * * * * * * * * * * * * * * * *$ 


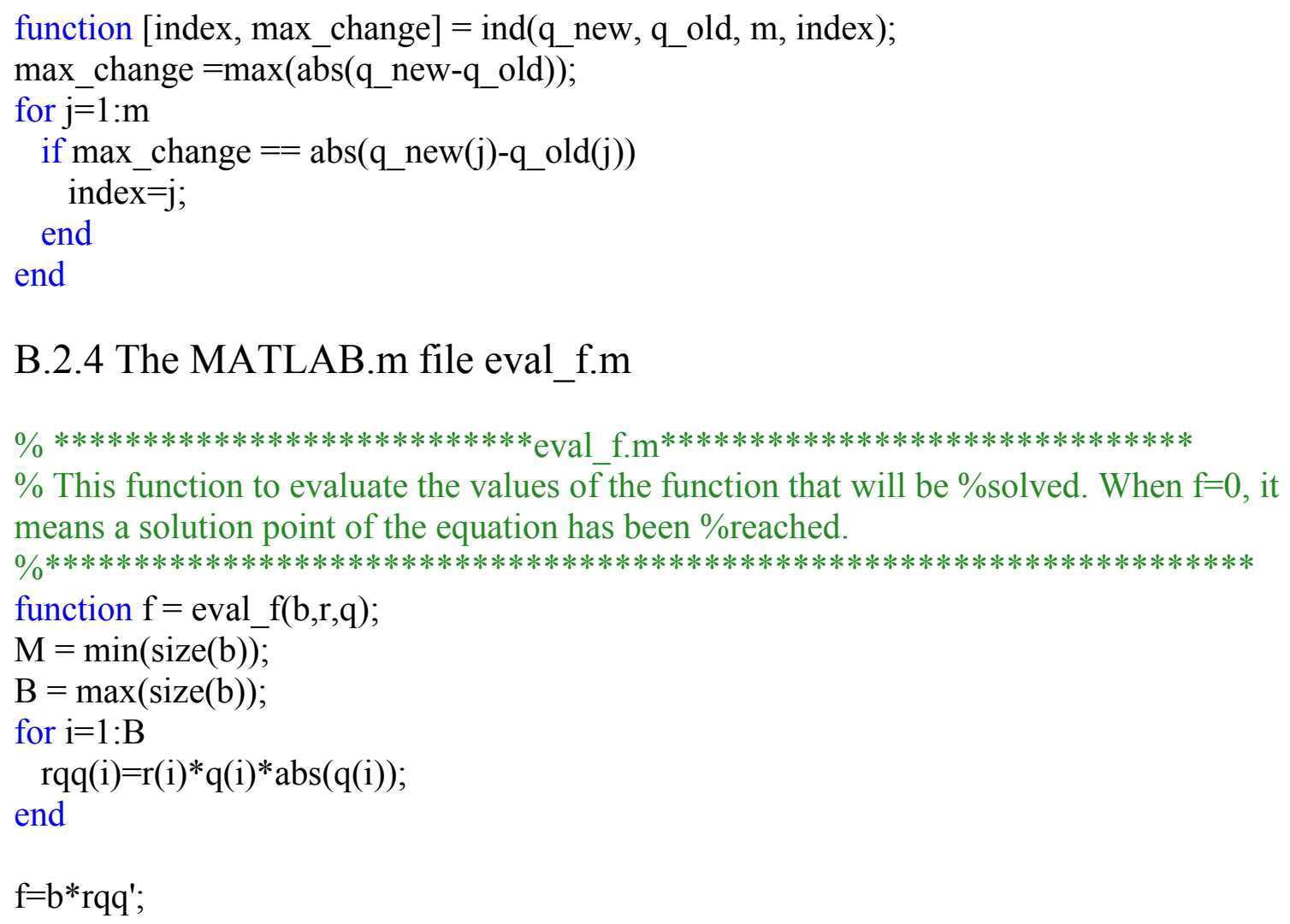

\section{B.2.5 The MATLAB.m file t1.m}

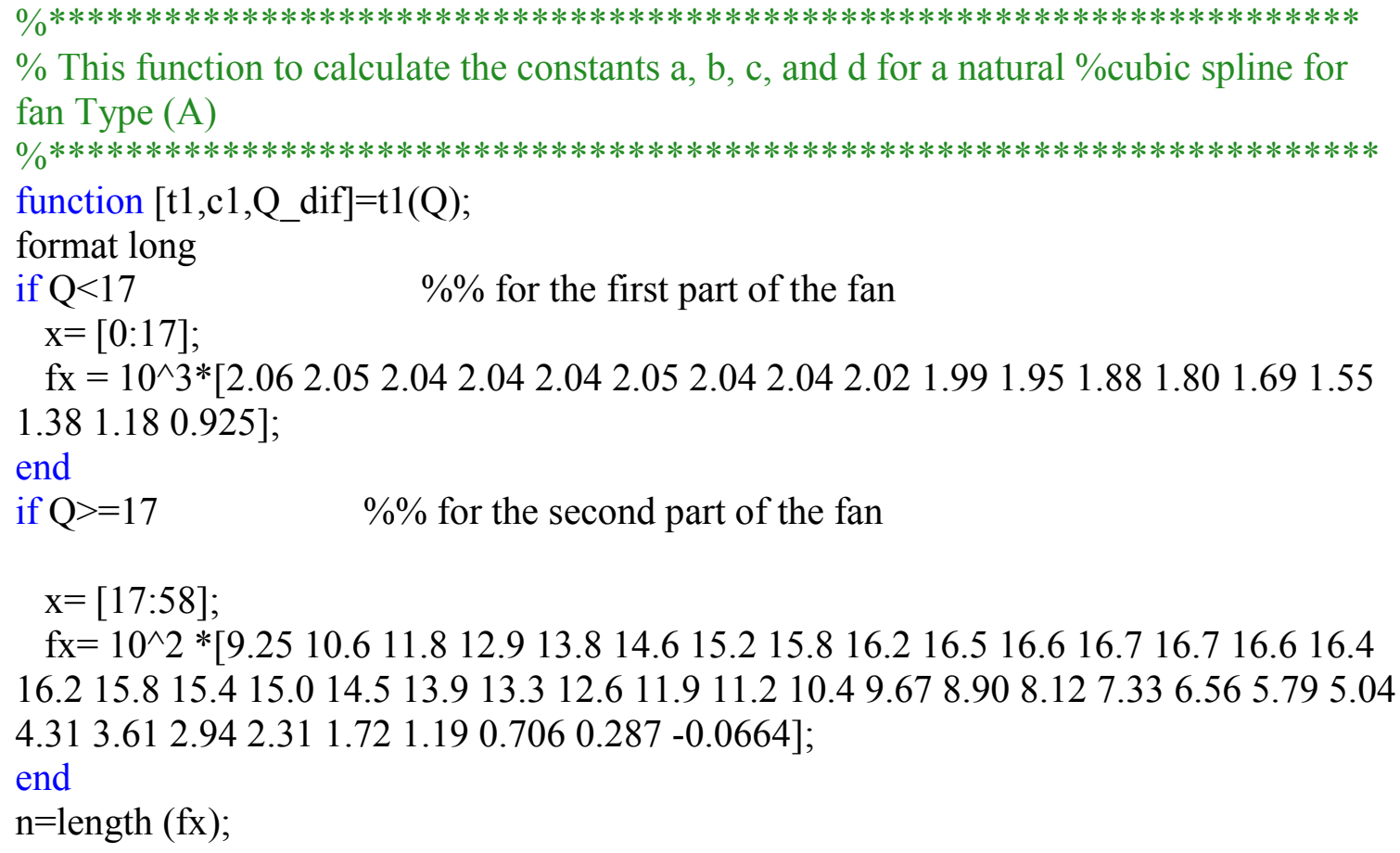




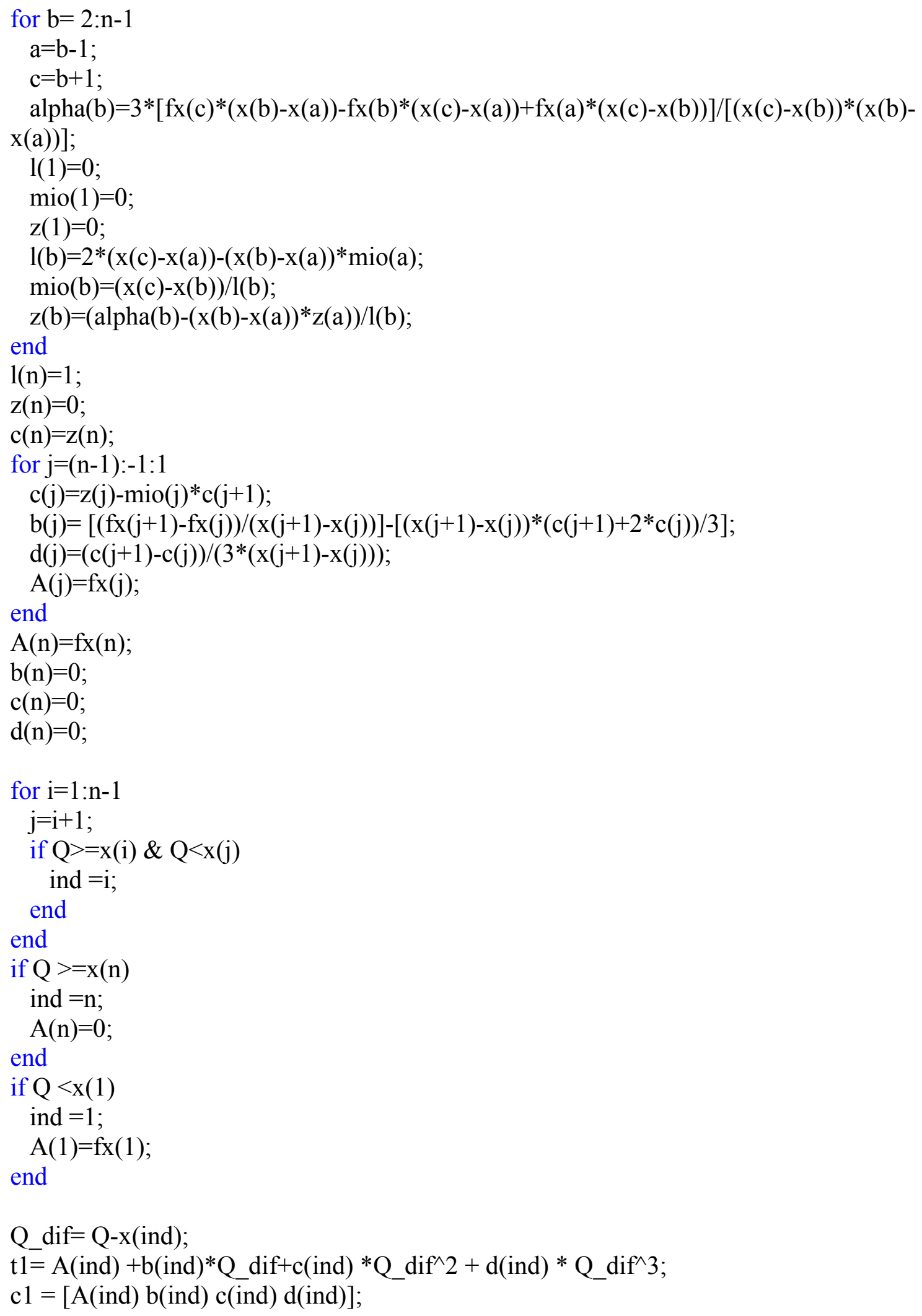




\section{B.2.6 The MATLAB.m file t2.m}

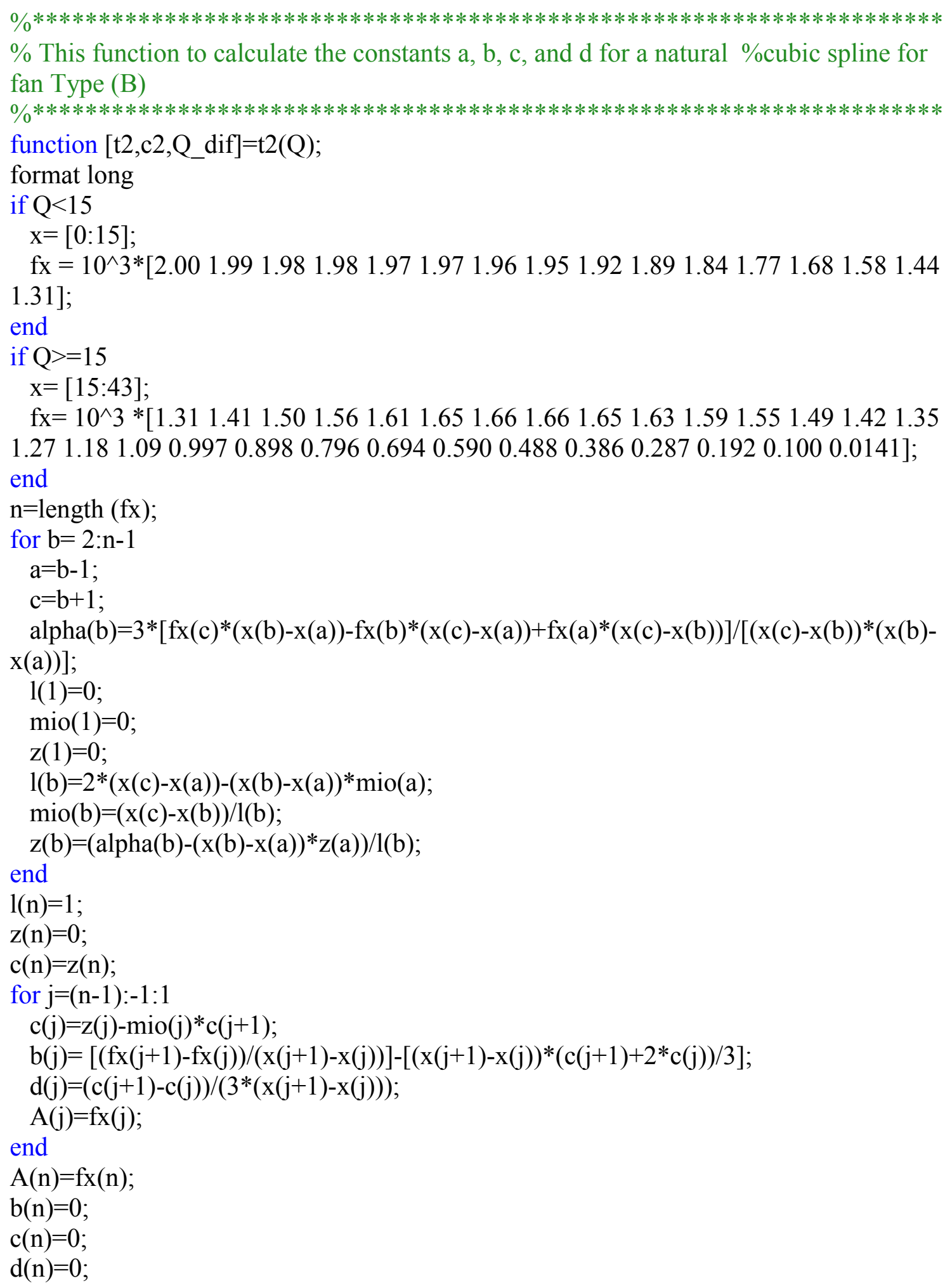




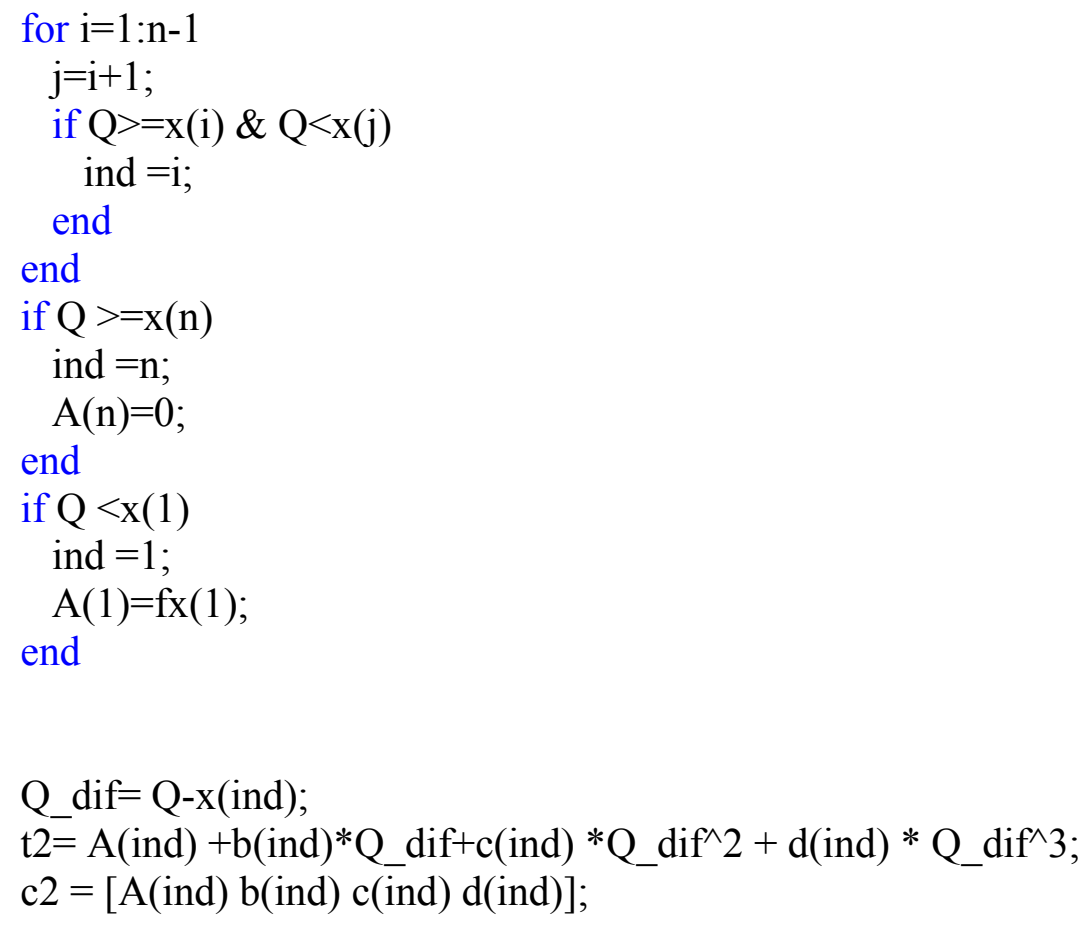

\section{B.2.7 The MATLAB.m file try $1 . m$}

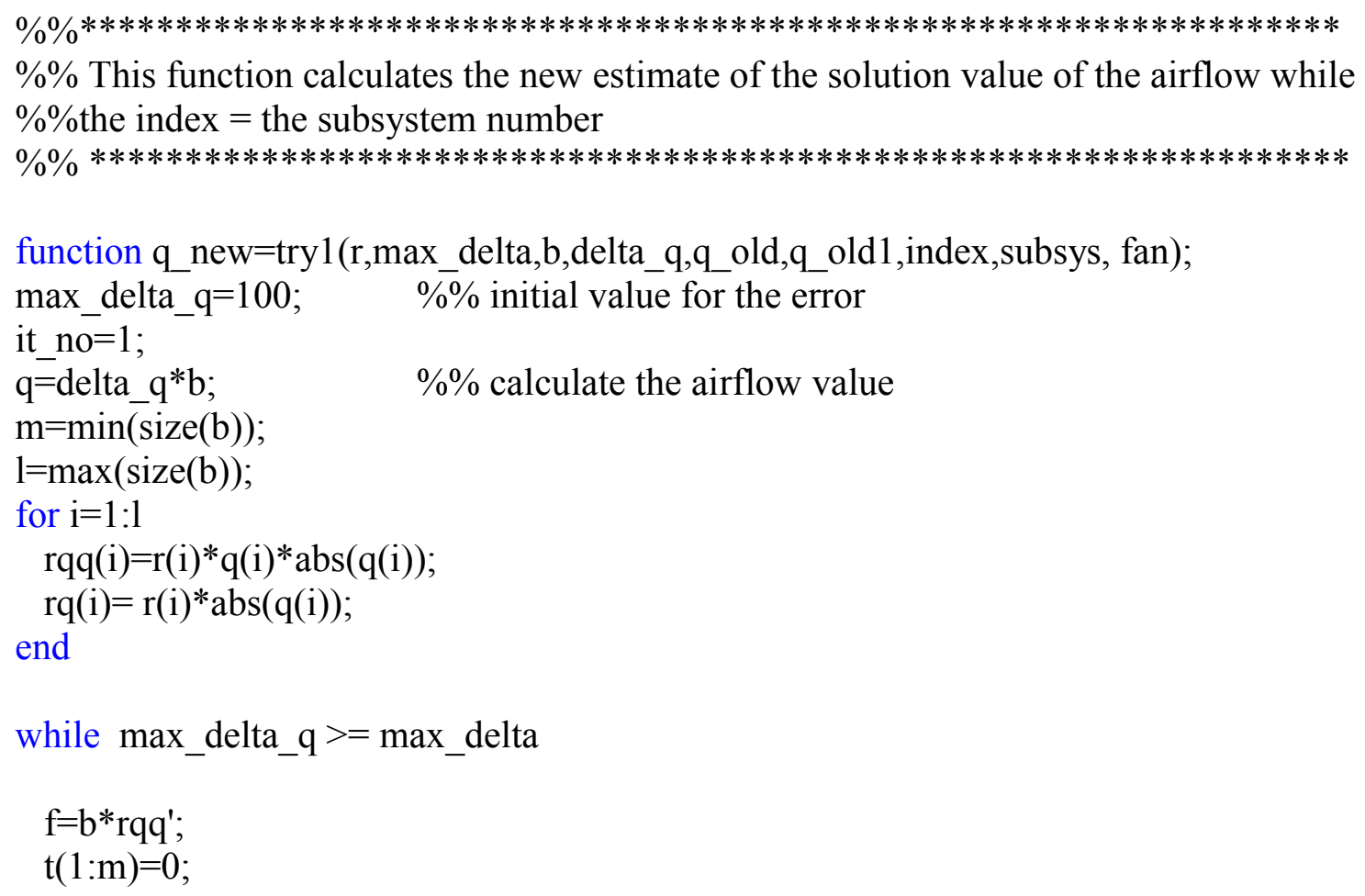




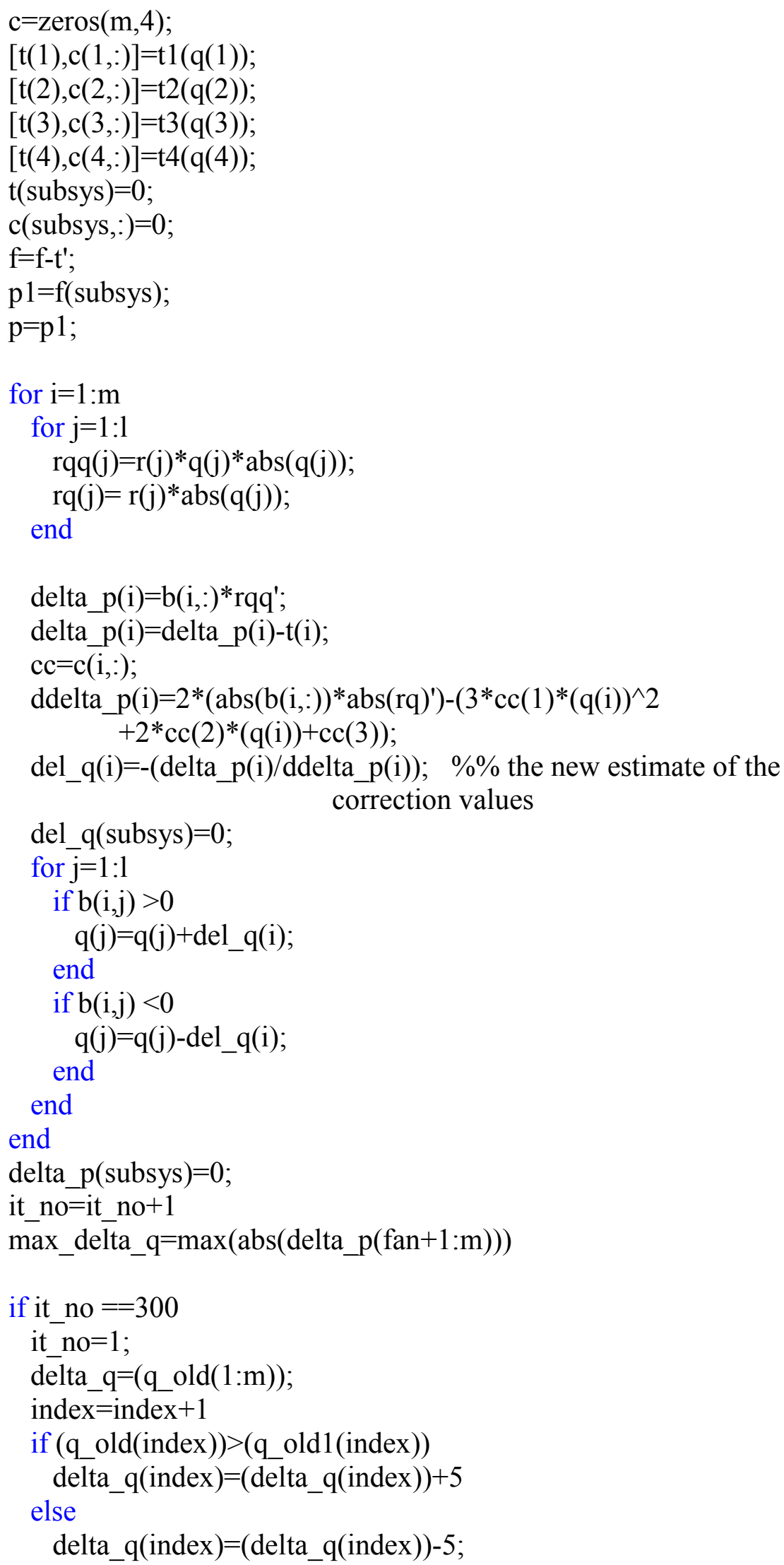




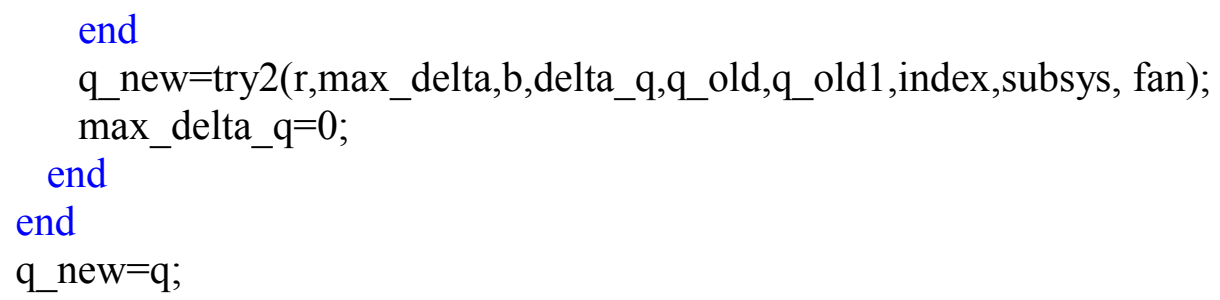

B.2.8 The MATLAB.m file try2.m

$\% \% * * * * * * * * * * * * * * * * * * * * * * * * * * * * * * * * * * * * * * * * * * * * * * * * * * * * * * * * * * * * * 0 \% \%$

$\% \%$ This function calculates the new estimate of the solution value of the airflow while the index does not equal to the subsystem and it is less than the fan number.

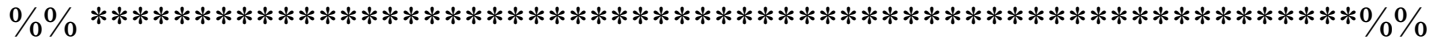

function q_new=try2(r,max_delta,b,delta_q,q_old,q_old1,index,subsys, fan);

max_delta_q=100; $\quad \% \%$ assign initial value for the error

it no $=1$;

$\mathrm{q}=$ delta_q*b; $\quad \% \%$ calculate the value of the airflows

$\mathrm{m}=\min (\operatorname{size}(\mathrm{b}))$;

$1=\max (\operatorname{size}(b))$;

for $\mathrm{i}=1: 1$

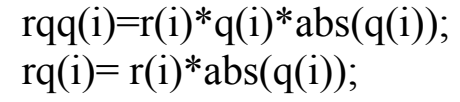




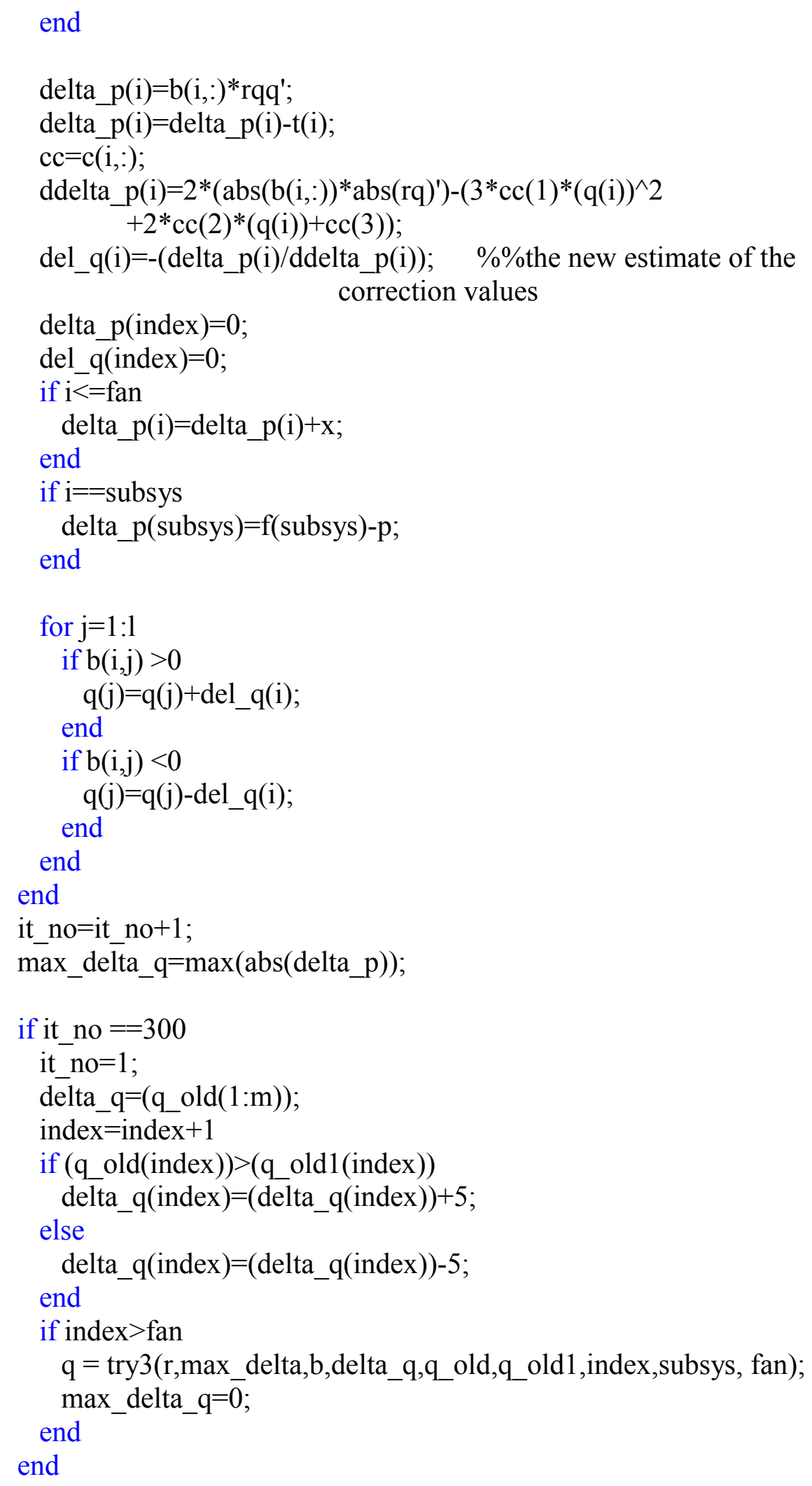


q_new $=\mathrm{q}$;

\section{B.2.9 The MATLAB.m file try3.m}

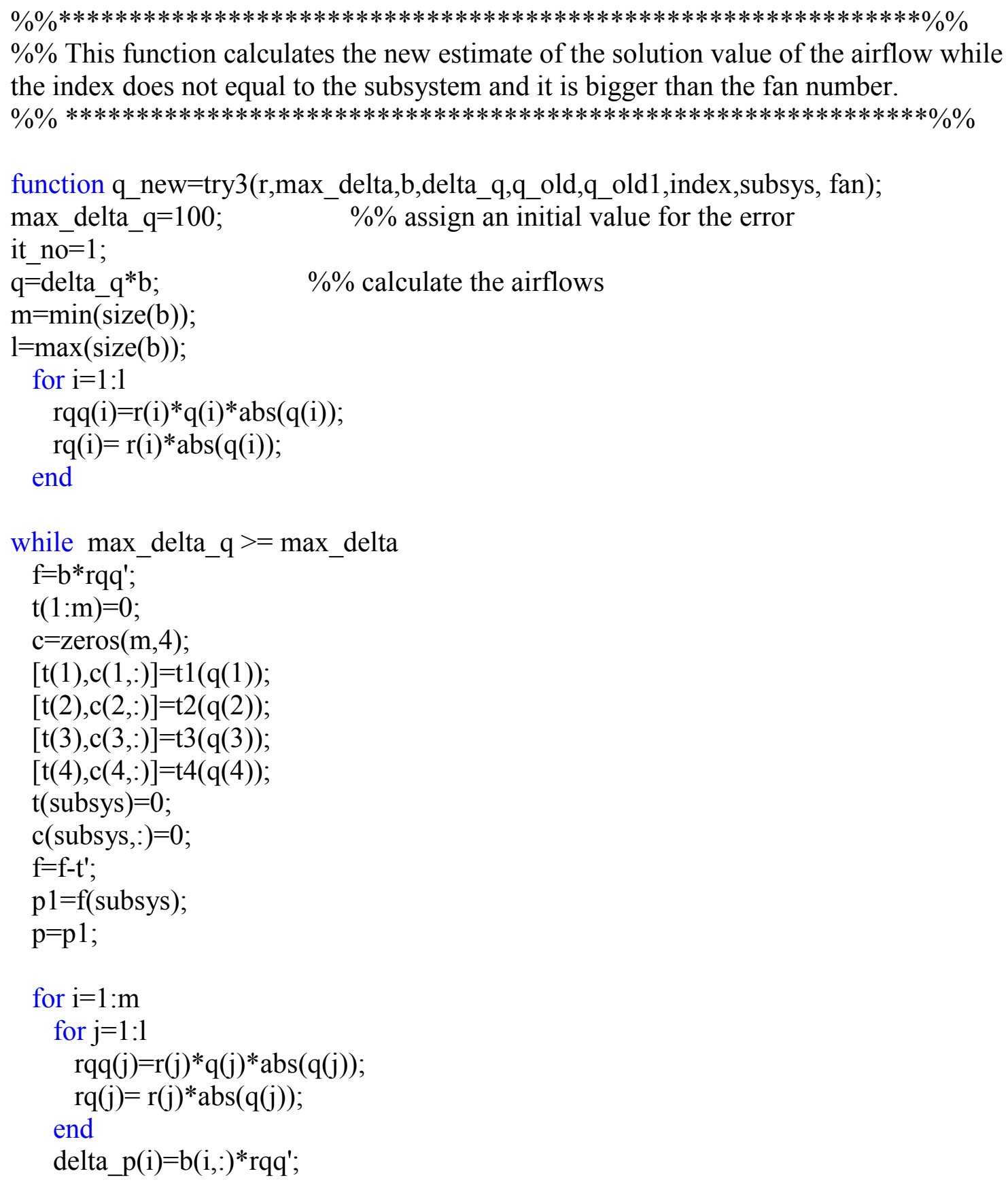




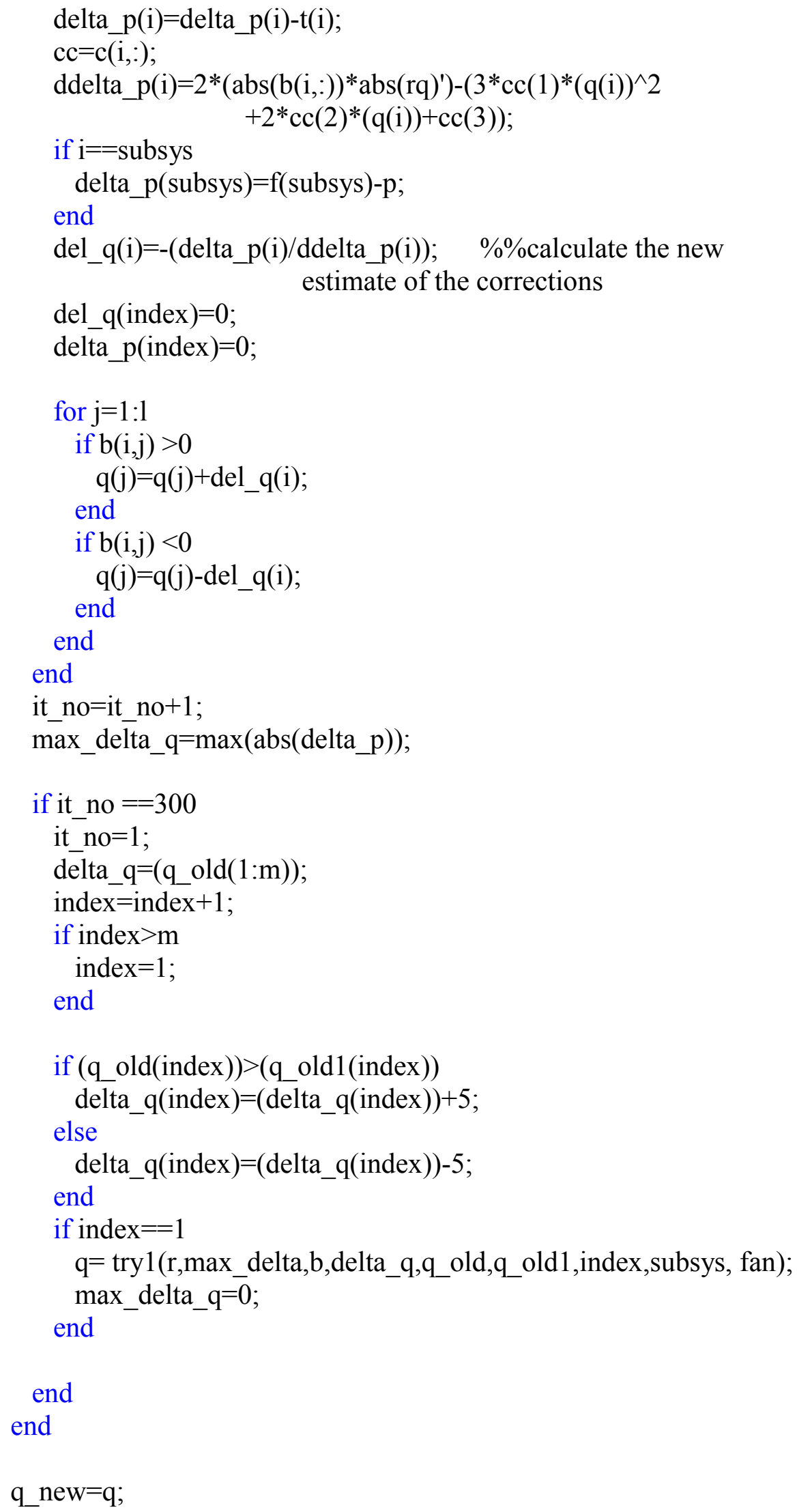




\section{B.3 The MATLAB.m file newton.m}

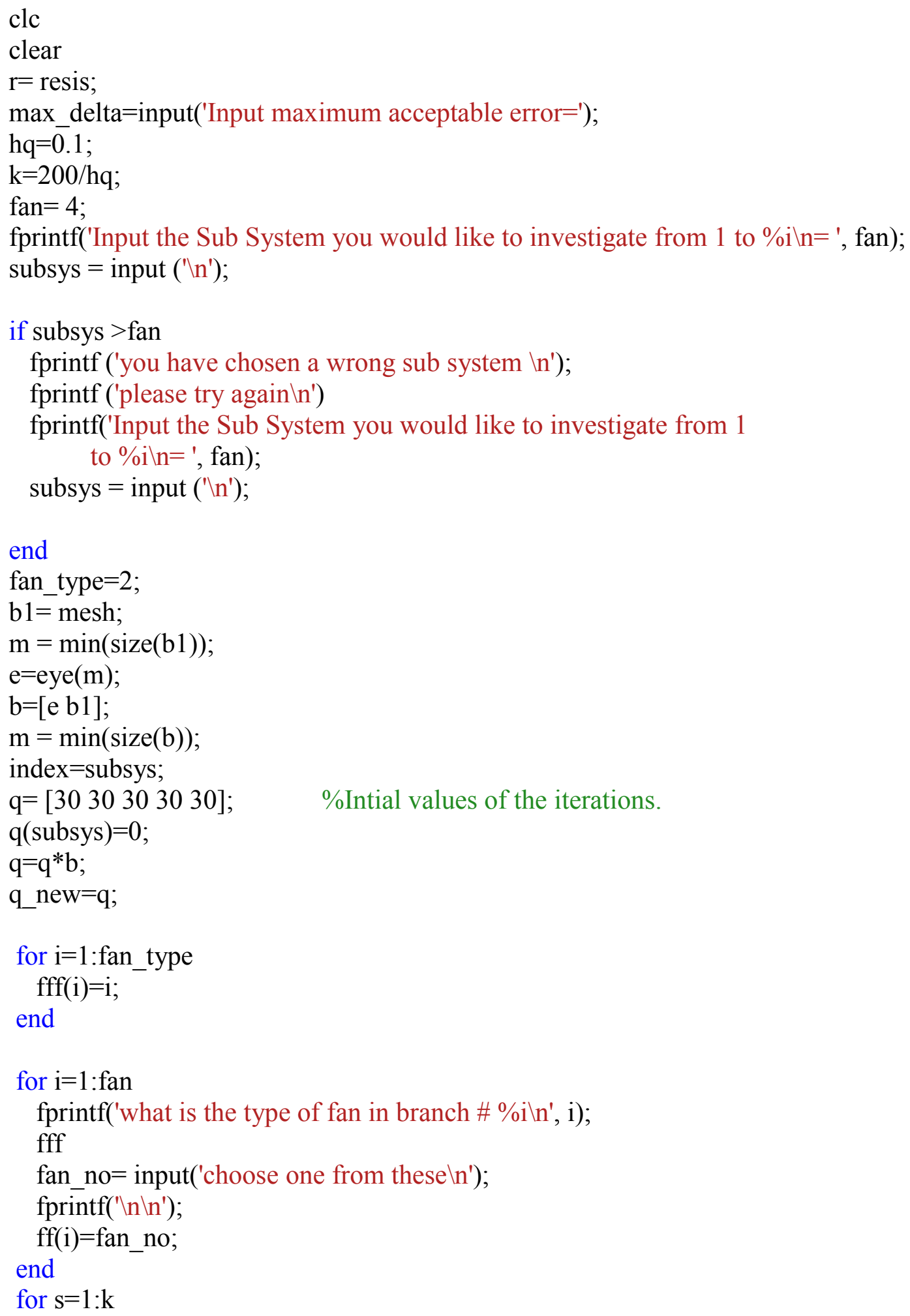




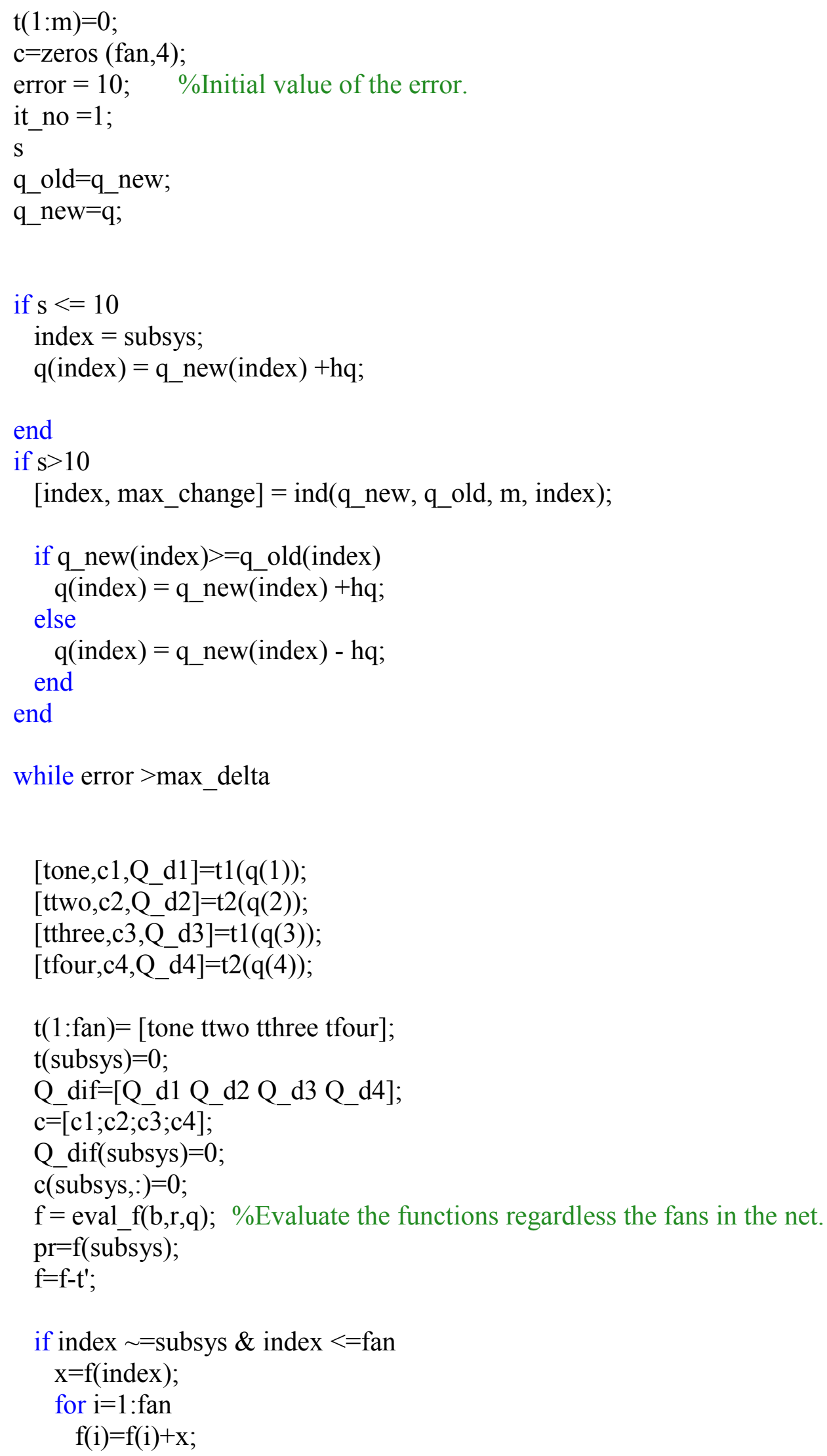




$$
\text { end }
$$

$\mathrm{f}($ subsys $)=\mathrm{f}($ subsys $)$-pr;

end

$\mathrm{f}($ index $)=0$;

$\mathrm{df}=\mathrm{jacob}\left(\mathrm{b}, \mathrm{r}, \mathrm{q}, \mathrm{c}, \mathrm{fan}, \mathrm{Q} \_\mathrm{dif}\right) ; \quad \%$ call function Jacob to evaluate the Jacobian matrix.

fixed $(1: \mathrm{m})=0$

fixed(index $)=1$;

$\operatorname{df}($ index,: $)=$ fixed;

delta=gauss(df,-f); $\quad \%$ Call function guass to eliminate the functions and get the new estimate of the solution.

$\mathrm{q}=\mathrm{q}(1: \mathrm{m})+2 * \mathrm{hq} *$ delta';

if it $n o==1$

$\mathrm{f}($ subsys $)=\mathrm{pr}$;

error $=10$;

end

$\mathrm{q}=\mathrm{q} * \mathrm{~b}$

$\mathrm{pp}=\mathrm{f}$;

del $=\left[\mathrm{pp} ; 75^{*}\right.$ delta $]$;

error $=\max (\operatorname{abs}(\mathrm{del}))$;

it_no $=$ it_no +1 ;

if it_no $>200$

it no

error

$\max (\operatorname{abs}($ delta $))$

end

if it no $==700$

it_no $=1$

$\mathrm{q}$ _new $($ index $)=\mathrm{q} \_$new (index $)$-hq;

inde $x=$ inde $x+1$;

if index $>$ fan

index $=1$;

end

index

$\mathrm{q}=\mathrm{q} \_$new 


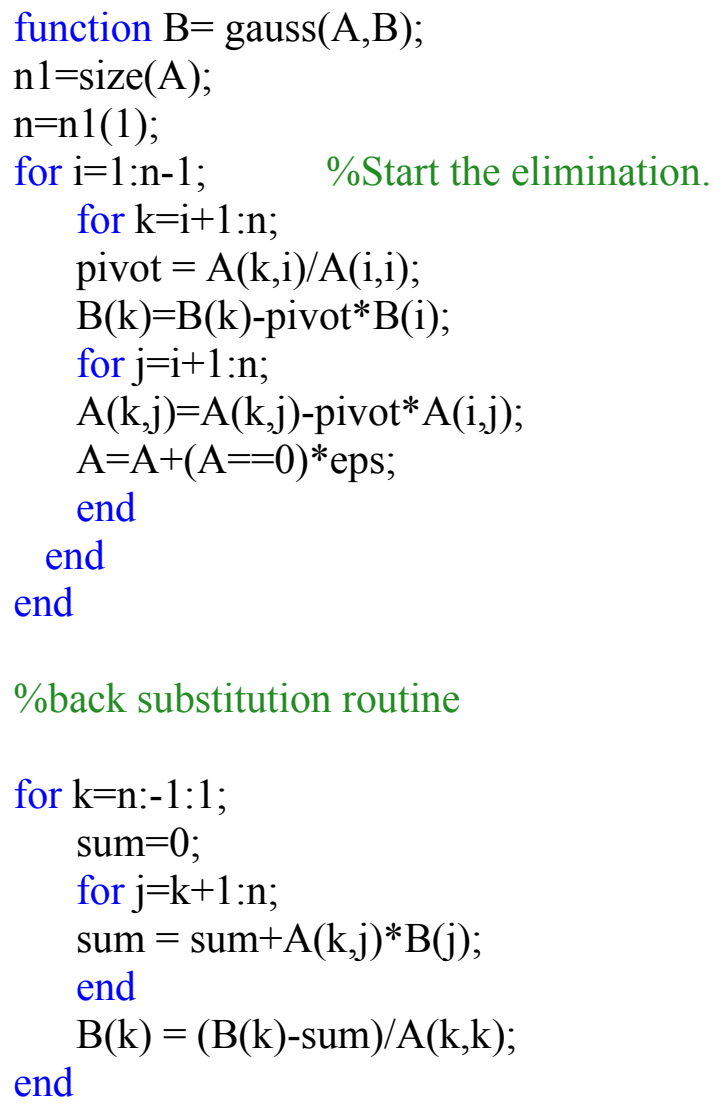

\section{B.3.2 The MATLAB.m file jacob.m}

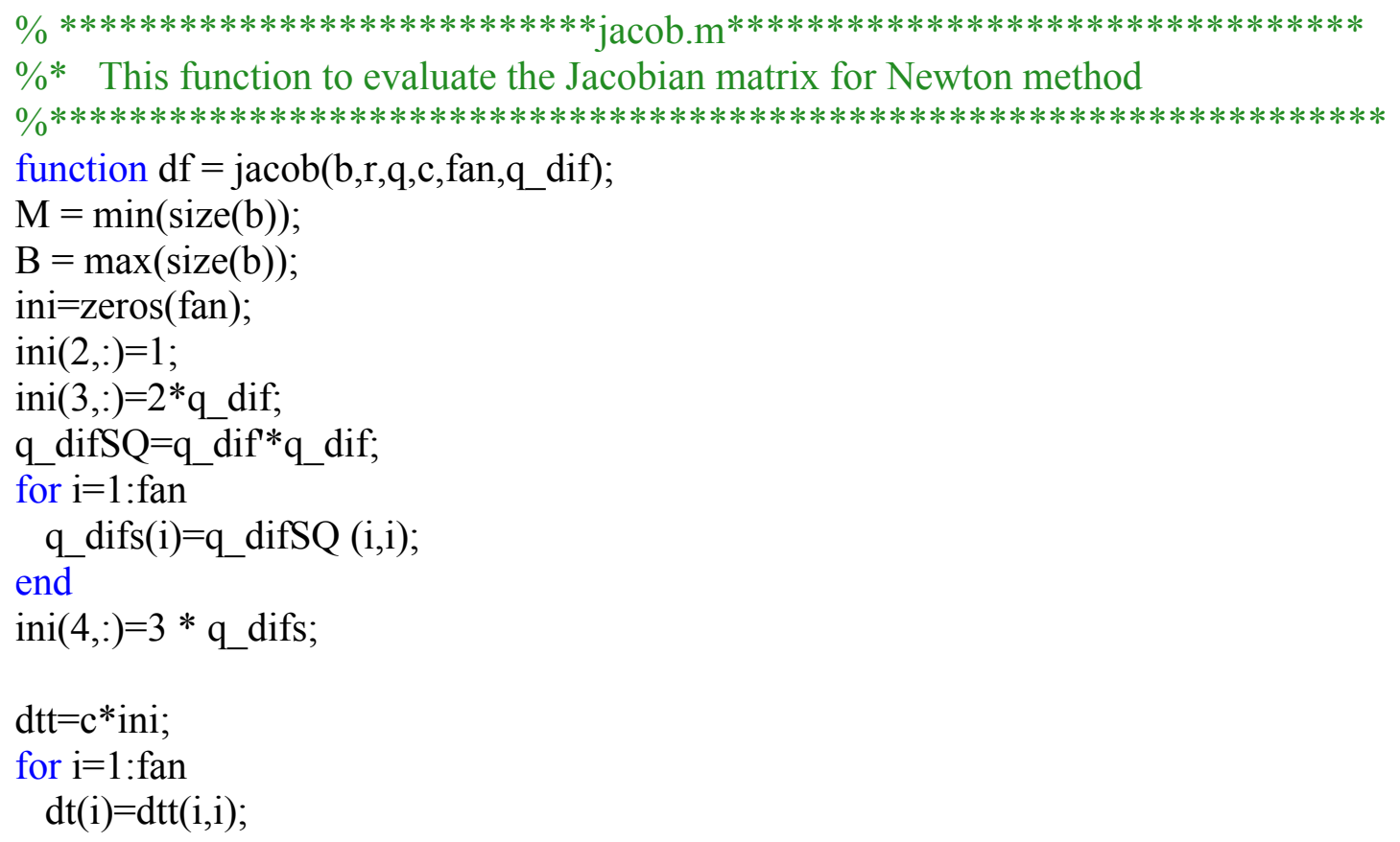




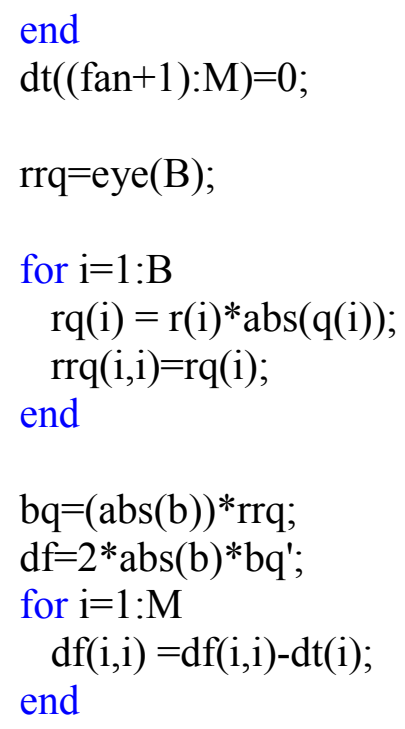

end 


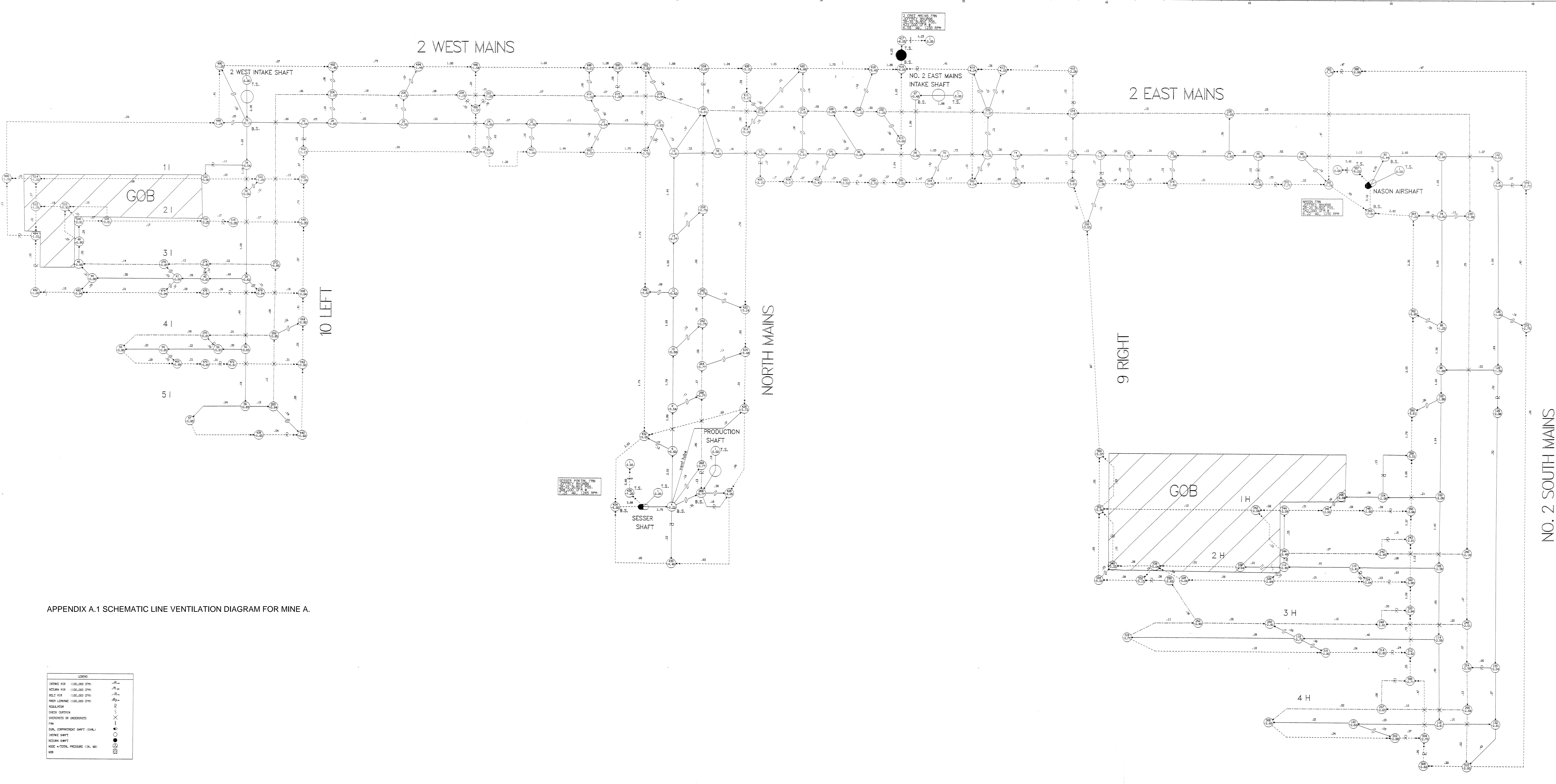




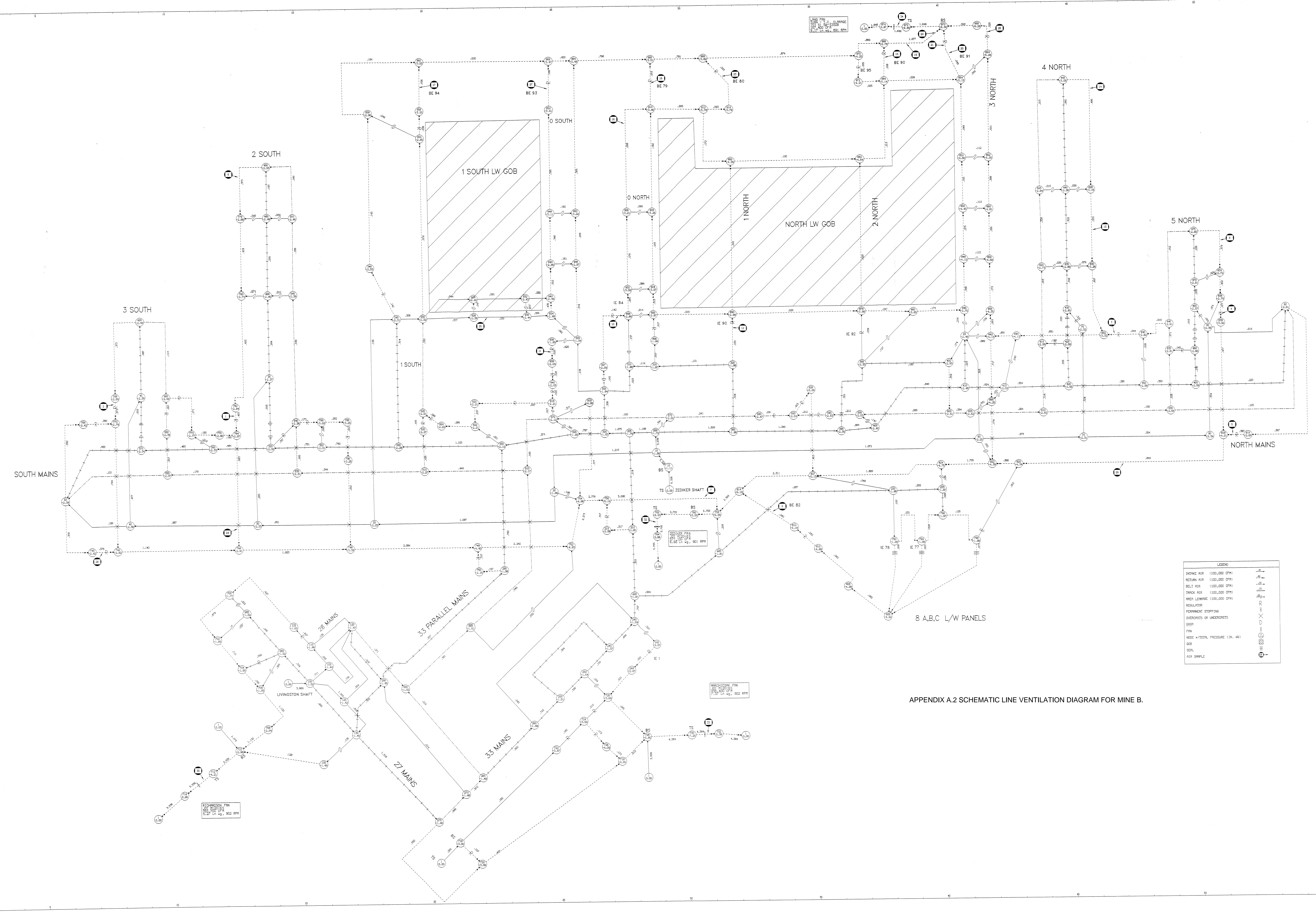






